Florida International University

FIU Digital Commons

FIU Electronic Theses and Dissertations

University Graduate School

6-23-2016

\title{
Conformational Dynamics and Stability Associated with Magnesium or Calcium Binding to DREAM in the Regulation of Interactions between DREAM and DNA or Presenilins
}

Khoa Ngoc Pham

Florida International University, kpham001@fiu.edu

DOI: 10.25148 /etd.FIDC000735

Follow this and additional works at: https://digitalcommons.fiu.edu/etd

Part of the Amino Acids, Peptides, and Proteins Commons, Biochemistry Commons, Biophysics Commons, Complex Mixtures Commons, Medicinal-Pharmaceutical Chemistry Commons, Molecular Biology Commons, and the Physical Chemistry Commons

\section{Recommended Citation}

Pham, Khoa Ngoc, "Conformational Dynamics and Stability Associated with Magnesium or Calcium Binding to DREAM in the Regulation of Interactions between DREAM and DNA or Presenilins" (2016). FIU Electronic Theses and Dissertations. 2589.

https://digitalcommons.fiu.edu/etd/2589 


\title{
FLORIDA INTERNATIONAL UNIVERSITY
}

Miami, Florida

\section{CONFORMATIONAL DYNAMICS AND STABILITY ASSOCIATED WITH MAGNESIUM OR CALCIUM BINDING TO DREAM IN THE REGULATION OF INTERACTIONS BETWEEN DREAM AND DNA OR PRESENILINS}

\author{
A dissertation submitted in partial fulfillment of \\ the requirements for the degree of \\ DOCTOR OF PHILOSOPHY \\ in \\ CHEMISTRY \\ by
}

Khoa Ngoc Pham 
To: Dean Michael R. Heithaus

College of Arts, Sciences and Education

This dissertation, written by Khoa Ngoc Pham, and entitled Conformational Dynamics and Stability Associated with Magnesium or Calcium Binding to DREAM in the Regulation of Interactions between DREAM and DNA or Presenilins, having been approved in respect to style and intellectual content, is referred to you for judgment.

We have read this dissertation and recommend that it be approved.

Xiaotang Wang

$\begin{array}{r}\text { Yuan Liu } \\ \hline \text { John P. Berry } \\ \hline \text { Prem P. Chapagain } \\ \hline \text { Jaroslava Miksovska, Major Professor }\end{array}$

Date of Defense: June 23, 2016

The dissertation of Khoa Ngoc Pham is approved.

Dean Michael R. Heithaus College of Arts, Sciences and Education

Andrés G. Gil Vice President for Research and Economic Development and Dean of the University Graduate School

Florida International University, 2016 
C Copyright 2016 by Khoa Ngoc Pham

All rights reserved. 


\section{DEDICATION}

This dissertation is dedicated to my lovely wife, Khanh Phan, and my beloved

daughter, Kimberly Pham. Without their love, motivation, inspiration, and support, this work could not have been completed. 


\section{ACKNOWLEDGMENTS}

I would like to acknowledge my major Professor, Dr. Jaroslava Miksovska, for giving me the great opportunity to work on this project. I also greatly appreciate Dr. Miksovska for her support and encouragement during my undergraduate and graduate research in her laboratory. I am grateful for her invaluable advice and discussions, which, I believe, greatly helped me to become an independent researcher.

I would also like to acknowledge my committee members Dr. Xiaotang Wang, Dr. Yuan Liu, Dr. John Berry, and Dr. Prem Chapagain for their helpful comments and supportive ideas to improve my research project and professional skills.

I would like to thank our collaborators Dr. Prem Chapagain, Dr. Bernard Gerstman, Dr. Chola Regmi, and Dr. Purushottam Tiwari in the Department of Physics at FIU, as well as Dr. Randy Larsen in the Department of Chemistry at University of South Florida for their successful and productive collaboration.

I also thank the former and present members in Dr. Miksovska's research group. I appreciate Dr. Luisana Astudillo, Dr. Walter Gonzalez, Simona Horsa, and Gangadhar Duhlipala for their support during my beginning in the laboratory and for our long friendship throughout these years. Thanks to David Butcher, Antonija Tangar, Jose Alfonso, Jessica Lopez, Andres Arango, Maria Santiago, Maurizio Diaz, Jackie Pivaral, Samiol Azam, and Ruben Fernandez for their helpful discussions and overall good times in the laboratory.

I am indebted to my parents for their love, dedication, and sacrifice to give me the best education as they could. I especially thank my brothers Dr. Si Pham and Binh Pham, and their families for the support and encouragement they provided during all these years. 
I would also like to give special thanks to my sisters Nga Pham, Thuy Pham, her husband Long Phi, and all my other brothers and sisters for the love and support they unconditionally provided.

Lastly, I highly appreciate the Florida International University (FIU) Graduate School of Integrated Science \& Humanity, College of Arts, Sciences \& Education, and Department of Chemistry and Biochemistry for providing me with graduate assistantship. I would like to acknowledge the financial support of the FIU Graduate School Dissertation Year Fellowship (2016). I would also like to acknowledge MBRS-RISE Biomedical Research Initiative (BRI) for providing me the 2014 BRI Student Summer Research award (NIH/NIGMSR25 GM061347). The content presented here is solely the responsibility of the author and does not necessarily represent the official views of the National Institutes of Health. 


\begin{abstract}
OF THE DISSERTATION
CONFORMATIONAL DYNAMICS AND STABILITY ASSOCIATED WITH

MAGNESIUM OR CALCIUM BINDING TO DREAM IN THE REGULATION OF

INTERACTIONS BETWEEN DREAM AND DNA OR PRESENILINS
\end{abstract}

by

Khoa Ngoc Pham

Florida International University, 2016

Miami, Florida

Professor Jaroslava Miksovska, Major Professor

Downstream regulatory element antagonist modulator (DREAM) is involved in various interactions with targets both inside and outside of the nucleus. In the cytoplasm, DREAM interacts with the C-terminal fragments of presenilins to facilitate the production of $\beta$-amyloid plaques in Alzheimer's disease. In the nucleus, $\mathrm{Ca}^{2+}$ free DREAM directly binds to specific downstream regulatory elements of prodynorphin/c-fos gene to repress the gene transcription in pain modulation. These interactions are regulated by $\mathrm{Ca}^{2+}$ and/or $\mathrm{Mg}^{2+}$ association at the EF-hands in DREAM. Therefore, understanding the conformational dynamics and stability associated with $\mathrm{Ca}^{2+}$ and/or $\mathrm{Mg}^{2+}$ binding to DREAM is crucial for elucidating the mechanisms of interactions of DREAM with DNA or presenilins. The critical barrier for envisioning the mechanisms of these interactions lies in the lack of NMR/crystal structures of Apo and $\mathrm{Mg}^{2+}$ DREAM.

Using a combination of fluorescence spectroscopy, circular dichroism, isothermal titration calorimetry, photothermal spectroscopy, and computational approaches, I showed that $\mathrm{Mg}^{2+}$ association at the EF-hand 2 structurally stabilizes the N-terminal alpha-helices 
1,2 , and 5 , facilitating the interaction with DNA. Binding of $\mathrm{Ca}^{2+}$ at the EF-hand 3 induces significant structural changes in DREAM, mediated by several hydrophobic residues in both the $\mathrm{N}$ - and C-domains. These findings illustrate the critical role of EF-hand 3 for $\mathrm{Ca}^{2+}$ signal transduction from the $\mathrm{C}$ - to $\mathrm{N}$-terminus in DREAM. The $\mathrm{Ca}^{2+}$ association at the EFhand 4 stabilizes the C-terminus by forming a cluster consisting of several hydrophobic residues in C-terminal domain. I also demonstrated that association of presenilin-1 carboxyl peptide with DREAM is $\mathrm{Ca}^{2+}$ dependent and the complex is stabilized by aromatic residues F462 and F465 from presenilin-1 and F252 from DREAM. Stabilization is also provided by residues R200 and R207 in the loop connecting $\alpha 7$ and $\alpha 8$ in DREAM and the residues D450 and D458 in presenilin-1.

These findings provide a structural basis for the development of new drugs for chronic pain and Alzheimer's disease treatments. 


\section{TABLE OF CONTENTS}

CHAPTER

PAGE

PREFACE 1

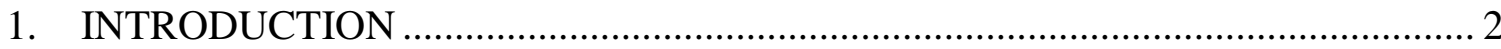

1.1 Calcium and intracellular $\mathrm{Ca}^{2+}$ signaling network ................................. 2

1.2 Intracellular calcium binding proteins ................................................... 4

1.3 Neuronal calcium sensor (NCS) proteins and the biological functions of $\mathrm{Ca}^{2+}$ binding to NCS proteins ............................................ 5

1.3.1 Class A: Neuronal calcium sensor 1 ..................................................... 12

1.3.2 Class B: Visinin-like proteins, hippocalcin, and neurocalcin .................. 13

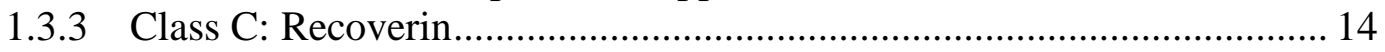

1.3.4 Class D: Guanylyl cyclase activating proteins..................................... 16

1.3.5 Class E: Potassium channel interacting proteins .................................. 17

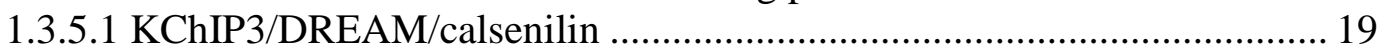

1.3.5.2 Structural insight from DREAM......................................................... 20

1.3.5.3 The $\mathrm{Ca}^{2+}$ and $\mathrm{Mg}^{2+}$ binding properties in DREAM .....................................24

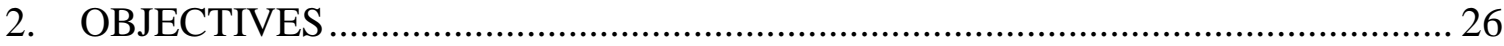

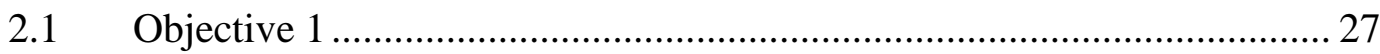

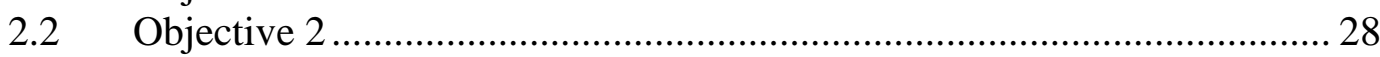

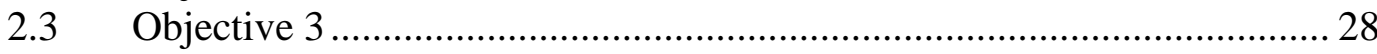

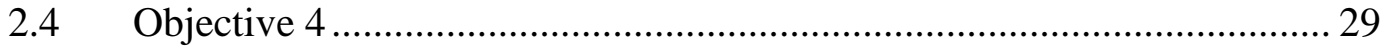

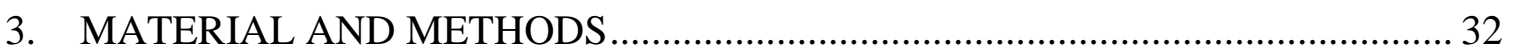

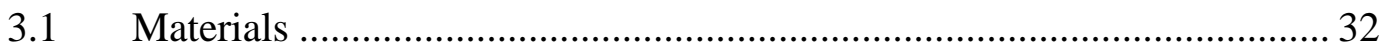

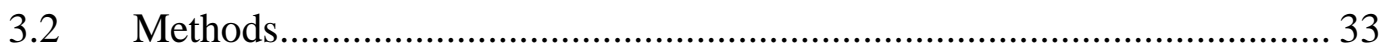

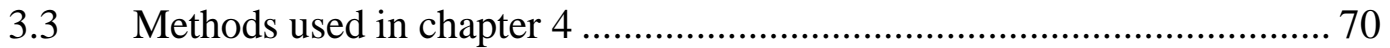

3.4 Methods used in chapter 5 ................................................................ 74

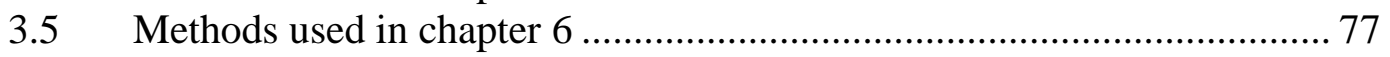

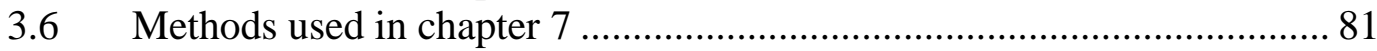

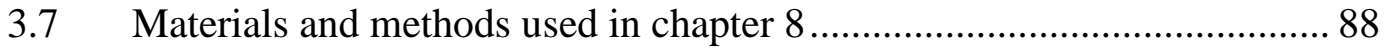

4. CALCIUM AND MAGNESIUM MODULATE CONFORMATIONAL DYNAMICS AND STABILITY OF DOWNSTREAM REGULATORY ELEMENT ANTAGONIST MODULATOR ....................................................... 91

4.1 Background and significance ....................................................... 91

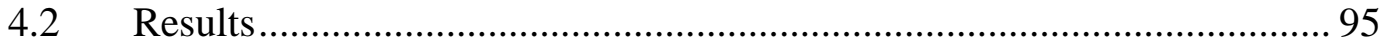

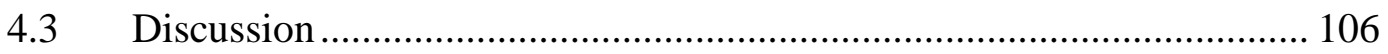

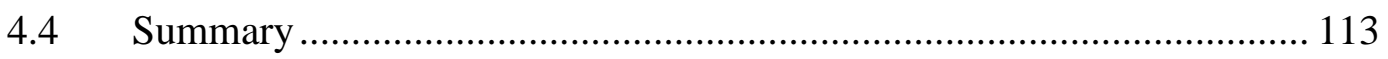


5. CALCIUM AND MAGNESIUM BINDING TO THE C-TERMINAL DOMAIN MODULATES DREAM STRUCTURE, DYNAMICS, AND

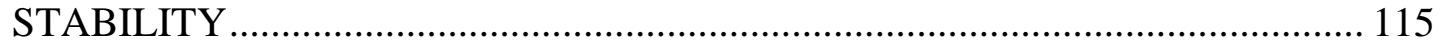

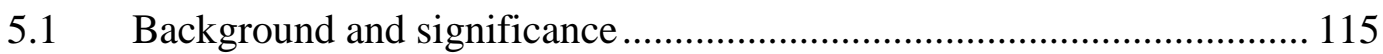

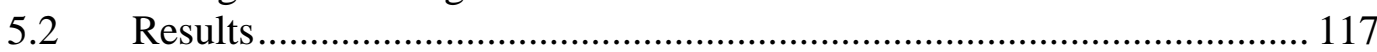

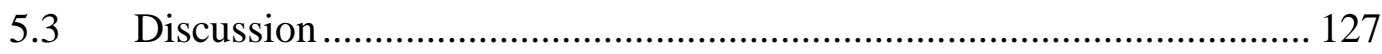

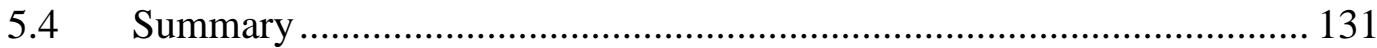

6. MOLECULAR INSIGHTS INTO DREAM AND PRESENILIN 1 C-

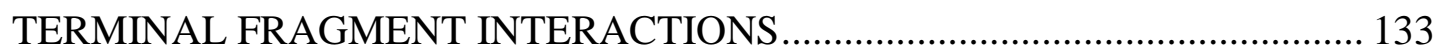

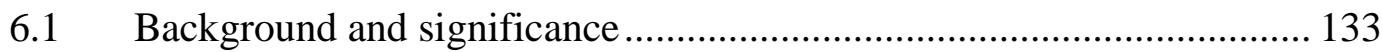

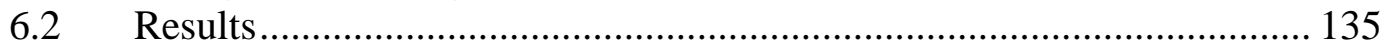

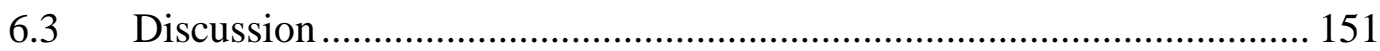

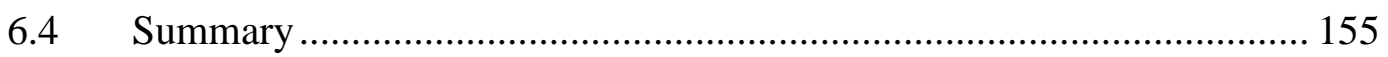

7. THE ROLE OF INDIVIDUAL EF-HANDS IN MODULATING DREAM CONFORMATIONAL DYNAMICS AND INTERACTION WITH DNA ........... 156

7.1 Background and significance .......................................................... 156

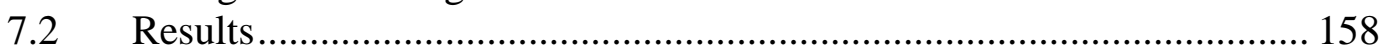

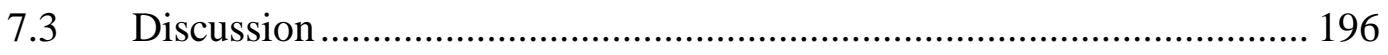

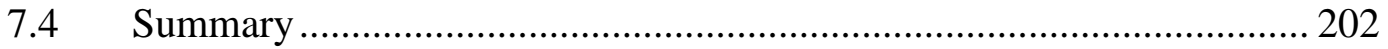

8. CHARACTERIZE KINETICS AND THERMODYNAMICS ASSOCIATED WITH CARBON MONOXIDE BINDING OR RELEASING FROM HORSE HEART MYOGLOBIN AND MICROPEROXIDASE-11 IN SODIUM BIS(2-ETHYLHEXYL) SULFOSUCCINATE ENCAPSULATION: A MODEL SYSTEM TO STUDY CONFORMATIONAL DYNAMICS OF DREAM FULL LENGTH IN MEMBRANE ANCHORED STATE. ............................... 203

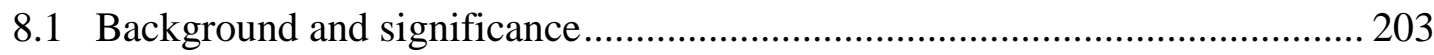

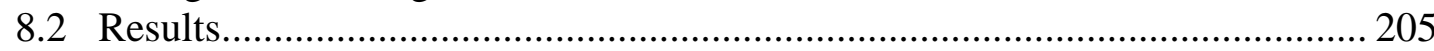

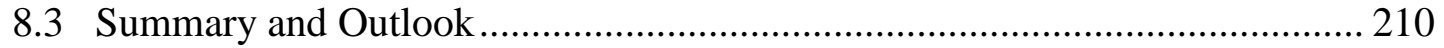

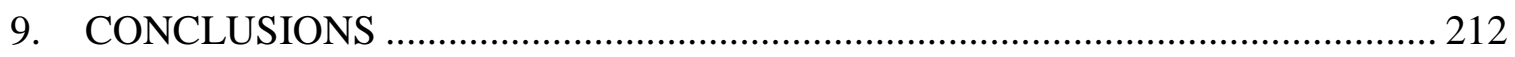

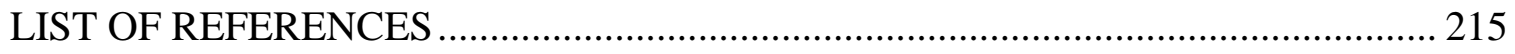

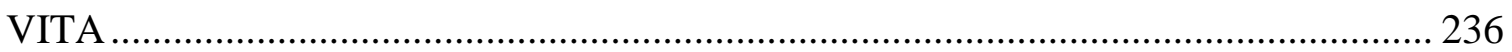




\section{LIST OF TABLES}

TABLE

PAGE

Table 3.1 Chemical components for electrophoresis running buffer. 35

Table 3.2 Chemical components for sample treatment buffer 36

Table 3.3 DREAM protein extinction coefficient at $280 \mathrm{~nm}$ .36

Table 3.4 List of extinction coefficients $(\varepsilon \lambda \mathrm{nm})$, emission maximum wavelength $\left(\right.$ Em. $\left.\lambda_{\max }\right)$ of fluorophores used 59

Table 3.5 Volumes and concentrations of protein and chemicals used to prepare CD samples of Apo, $\mathrm{Mg}^{2+}, \mathrm{Ca}^{2+}$, and $\mathrm{Ca}^{2+} \mathrm{Mg}^{2+}$ bound DREAM at identical concentration of DREAM protein

Table 4.1 Stern-Volmer constant $\left(\mathrm{K}_{\mathrm{sv}}\right)$ and bimolecular quenching constant $\left(\mathrm{k}_{\mathrm{q}}\right)$ of DREAM protein

Table 4.2 Emission decay parameters for DREAM using a continuous Gaussian distribution and a discrete component

Table 4.3 Thermodynamic parameters for $\mathrm{GuHCl}$ induced denaturation of DREAM in the absence/presence of $\mathrm{Ca}^{2+}$ and $\mathrm{Mg}^{2+}$.

Table 5.1 Thermodynamic parameters for $\mathrm{Ca}^{2+}$ and $\mathrm{Mg}^{2+}$ binding to DREAM-C....... 119

Table 5.2 The Stern-Volmer constants $\left(\mathrm{K}_{\mathrm{sv}}\right)$ for acrylamide quenching of DREAM-C

Table 5.3 Fluorescence decay parameters for DREAM-C intrinsic tryptophan residue. Data were analyzed using a Gaussian-discrete model.

Table 5.4 Fluorescence decay parameters for nile red:DREAM-C. The experimental data were analyzed using a discrete component and continuous Gaussian distribution model.

Table 5.5 Thermodynamic parameters for DREAM-C unfolding

Table 6.1 Dissociation constant $\left(\mathrm{K}_{\mathrm{d}}\right)$ of DREAM binding to HL $\beta$ and HL9 in PS1-

$\mathrm{CTF}$

Table 6.2 Dissociation constant $\left(\mathrm{K}_{\mathrm{d}}\right)$ of DREAM-C binding to HL9 in PS1-CTF...... 139 
Table 6.3 Dissociation constant $\left(\mathrm{K}_{\mathrm{d}}\right)$ for HL9 binding to DREAM EF-hand mutants

Table 6.4 Kinetic parameters for HL9 association with $\mathrm{Ca}^{2+}$ DREAM

Table 7.1 Emission decay parameters of W169 in DREAM EF-hand mutants using a continuous Gaussian distribution and a discrete component.

Table 7.2 Decay parameters for 1,8-ANS and DREAM EF-hand mutant complexes

Table 7.3 ITC parameters recovered for DNA binding to DREAM and DREAM EF-hand mutants using a two sets of sites model

Table 7.4 Time-resolved anisotropy decay parameters of DREAM and DNA interaction

Table 8.1 The position of the Soret band and Q bands for Mb, and MP-11 encapsulated within the AOT reverse micelles, $\mathrm{w}_{\mathrm{o}}=40$. The numbers is parenthesis corresponds to the $\lambda_{\max }$ for $\mathrm{Mb}$ and MP-11 in aqueous solutions, $\mathrm{pH} 7.0$ 


\section{LIST OF FIGURES}

FIGURE

PAGE

Figure 1.1 Scheme illustrating a common intracellular $\mathrm{Ca}^{2+}$ signaling network. Adapted from (Berridge et al., 2000)

Figure 1.2 A single EF-hand 2 of recoverin (PDB code: 4YI8). The EF-hand consists of an entering $\alpha$-helix, a $\mathrm{Ca}^{2+}$ binding loop, and an exiting $\alpha$ helix. The $\mathrm{Ca}^{2+}$ atom (green sphere) is coordinated by a canonical $\mathrm{Ca}^{2+}$ binding loop illustrating the pentagonal bipyramidal coordination of the $\mathrm{Ca}^{2+}$ ion (purple lines) and the hydrogen bonding found in the loop (black lines). The side chain oxygen atoms are in red and the coordinating oxygen atoms from water are shown as red spheres.

Figure 1.3 The sequence of a canonical $\mathrm{Ca}^{2+}$ binding loop. The $\mathrm{Ca}^{2+}$ ligands are indicated by their positions in the loop. The ninth $\mathrm{Ca}^{2+}$ ligand (-X coordinate) is typically provided by a water molecule. Adapted from reference (Gifford et al., 2007).

Figure 1.4 N-terminal domain of recoverin contains a pair of EF-hands (PDB code: 4YI8, residues: 1-94). Alpha helices, denoted by $\alpha, 1$ to 5 are respectively labeled. EF-hand 1 is composed of $\alpha 2$ and $\alpha 3$; likewise, EF-hand 2 contains $\alpha 4$ and $\alpha 5$. $\mathrm{Ca}^{2+}$ is shown as a green sphere.

Figure 1.5 Representation of phylogenetic relationships in NCS proteins. Adapted from (Burgoyne et al., 2001).

Figure 1.6 Sequence alignment of NCS proteins from classes A to E. Conserved residues are represented by a star, whereas asterisks indicate identical residues, colons indicate very similar residues, and periods are for weakly similar residues.

Figure 1.7 Top: Schematic diagram of EF-hands in NCS1 protein. Bottom left: structure of myristoylated ApoNCS1 (PDB code: 2L2E). Bottom right: structure of three $\mathrm{Ca}^{2+}$ bound at EF-hand 2, 3, and 4 of nonmyristoylated NCS-1 (PDB code: 2LCP). The EF-hands 1, 2, 3, and 4 of NCS1 are colored in cyan, green, yellow, and red, respectively. The $\mathrm{Ca}^{2+}$ ions are the yellow spheres and the myristoyl group is colored in pink..

Figure 1.8 The crystal structure of $\mathrm{Ca}^{2+}$ bound non-myristoylated neurocalcin (PDB: 1BJF (Vijay-Kumar, 1999)). The EF-hands 1-4 are labeled accordingly as EF-1, EF-2, EF-3, and EF-4. The $\mathrm{Ca}^{2+}$ ion (yellow 
sphere) is bound at EF-2, EF-3, and EF-4. The EF-1 is disabled from binding of $\mathrm{Ca}^{2+} / \mathrm{Mg}^{2+}$ due to a Cys-Pro sequence in its $\mathrm{Ca}^{2+}$ binding loop

Figure 1.9 Top: Schematic diagram of EF-hands in recoverin. Bottom: (a) structure of myristoylated ApoRecoverin (PDB code: 1IKU), (b) 1 $\mathrm{Ca}^{2+}$ ion bound at EF-3 (PDB code: 1 OMR), and (c) $\mathrm{Ca}^{2+}$ ion is bound at EF-hands 2 and 3 (PDB code: 1JSA). The structure of two $\mathrm{Ca}^{2+}$ bound non-myristoylated recoverin is shown in light blue in (c) (PDB code: 4YI8). EF-hands 1, 2, 3, and 4 in recoverin are colored in cyan, green, yellow, and red, respectively. The $\mathrm{Ca}^{2+}$ ions (yellow spheres) are bound at EF-hand 2 and 3. The myristoyl group is shown as pink spheres and the N-terminal eight-residue-flexible loop is highlighted in yellow.

Figure 1.10 Top: Schematic diagram of EF-hands in GCAPs. Bottom: (left) structure of $\mathrm{Mg}^{2+}$ bound at EF-hand 2 of myristoylated GCAP 1 mutant V77E $\left(\mathrm{Mg}^{2+}\right.$ is not shown, PDB code: 2NA0) and (right) structure of $\mathrm{Ca}^{2+}$ bound at EF-hands 2, 3, and 4 in GCAP 1 (PDB code: 2R2I). EF-hands 1 to 4 are highlighted in cyan, green, yellow, and red, respectively. The $\mathrm{Ca}^{2+}$ ions are shown as yellow spheres

Figure 1.11 KChIPs and splice variants in class E. Some KChIP isoforms have myristoylation or palmitoylation sites, assisting membrane association and localization. The GenBank accession number for human KChIP1 isoforms 1.1, 1.2, 1.3 DQ148478, DQ148477, and DQ148476. The GenBank accession number for human KChIP2 isoforms 2.1, 2.2, 2.3, 2.4, and 2.5 are NM-01491, DQ148480, DQ148481, DQ148482, and DQ148483. The GenBank accession number for human KChIP3 isoforms 3.1 and 3.2 are DQ148485 and DQ148486. The GenBank accession number for human KChIP4 isoforms are DQ148487, DQ148488, DQ148491, DQ148489, DQ148490, and DQ148492, respectively. Adapted from (Burgoyne, 2007).

Figure 1.12 The amino acid sequence of mouse DREAM (Gene code: Q9QXT8). The $\mathrm{Ca}^{2+}$ and/or $\mathrm{Mg}^{2+}$ binding loops of EF-hands 1, 2, 3, and 4 are underlined. The negatively charged amino acids involved in $\mathrm{Ca}^{2+} / \mathrm{Mg}^{2+}$ coordination are shown in red. The conserved sequence CPxG (in brown) in the first EF-hand eliminates binding of $\mathrm{Ca}^{2+} / \mathrm{Mg}^{2+}$ at this site. The flexible loop connecting $\alpha 7$ from EFhands 3 and $\alpha 8$ from EF-hand 4 is shown in light green and underlined, while the positively residues R200 and R207 in the loop are shown in blue. The leucine residues (155LxxLL159, and L251) at $\mathrm{Ca}^{2+}$ DREAM dimeric interface are highlighted in purple. 
Figure 1.13 Three dimensional cartoon structure of $\mathrm{Ca}^{2+}$ DREAM (PBS: 2JUL, residues 76 - 256) (Lusin et al., 2008). The structure DREAM consists of $10 \alpha$-helices ( $43 \%$ secondary structure content) connected by random coils, turns, and a few beta sheets ( $2 \%)$. From left to right, the positively charged residues postulated to facilitate DNA binding (K87, K90, K91, R98, K101, R160) are shown in licorice. The EFhands $1,2,3$, and 4 are shown in cyan, green, yellow, and orange, respectively. The $\mathrm{N}$-terminal $\alpha$-helix $(\alpha 1)$ and the $\mathrm{C}$-terminal alpha helix $(\alpha 10)$ are labeled in blue and red, respectively. The single intrinsic tryptophan residue (W169) is shown in yellow licorice. The long flexible loop connecting $\alpha 7$ and $\alpha 8$ is shown in blue. The $\mathrm{Ca}^{2+}$ ion (yellow sphere) is bound to EF-hands 3 and 4 .

Figure 1.14 The central hydrophobic cluster (shown in stick) connecting the Nand the C-terminal domains of DREAM (PDB: 2JUL) (Lusin et al., 2008).

Figure 1.15 The C-terminal hydrophobic cluster (as shown in stick) of DREAM (Lusin et al., 2008).

Figure 3.1 The Perrin-Jablonski diagram. The arrows represent the transition of a quantum change. The transitions begin at the lowest vibrational levels of each electronic state $\left(S_{0}, S_{1}\right.$, and $\left.S_{2}\right)$ for both absorption (blue arrows) and de-excitation (green arrows). The transition from the excited state to the ground state always happens from the first lowest level of $\mathrm{S}_{1}$. The non-emissive de-excitation transitions are internal conversion (ic), intersystem crossing (isc), excited state reaction, quenching, photolysis, and FRET. The excited molecule passes from the singlet $\left(\mathrm{S}_{1}\right)$ to the triplet $\left(\mathrm{T}_{1}\right)$ state via isc. The emission from $\mathrm{T}_{1}$ is phosphorescence (red arrow). The other deexcitation transitions from $T_{1}$ are similar to that of the $S_{1}$. Adapted from (Lakowicz 2006; Periasamy and Clegg 2010)... .38

Figure 3.2 Relative phase shift $(\phi)$ and modulation of the excitation (subscript E) and emission light (subscript F) in FD fluorescence spectroscopy. $\mathrm{AC}$ and DC indicate the amplitude and offset of the respective waves. Adapted from (Ross and Jameson, 2008).

Figure 3.3 Schematic diagram for measurement of fluorescence anisotropy. Adapted from (Lakowicz, 2006).

Figure 3.4 Schematic diagram illustrating emission intensities for a single fluorophore in a coordinate system. Adapted from (Lakowicz, 2006) 
Figure 3.5 Schematic diagram for measurement of anisotropy decays with frequency-domain. Adapted from (Lakowicz, 2002).

Figure 3.6 Schematic diagram of a stopped-flow mixer. Reaction A and B are driven into the mixer through valves $\mathrm{A}$ and $\mathrm{B}$. The previous content of the tube and cuvette are flushed out the stopping syringe via valve $\mathrm{S}$ that is open during flow. When the plunger of the stopping syringe hits a block, the valve $\mathrm{S}$ is closed and the contents of the cuvette can be probed during reaction mixture. Adapted from (Hargrove, 2005)

Figure 3.7 Scheme representing linearly polarized light that is converted using a linear polarizer and the circularly polarized light that is created using a quarter-wave $(\lambda / 4)$ plate. Adapted from (Berova et al., 2000).

Figure 3.8 A: when circularly polarized light passes through achiral samples, its $\mathrm{E}_{\mathrm{L}}$ and $\mathrm{E}_{\mathrm{R}}$ components of are constant. B: when circularly polarized light passes through chiral samples, $E_{L}$ (blue) and $E_{R}$ (green) components are absorbed in distinctly, resulting in elliptical polarized light (red). Adapted from (Kelly et al., 2005)....

Figure 3.9 Far UV CD spectra associated with various types of secondary structure of proteins. Adapted from (Berova et al., 2000).

Figure 3.10 Near UV CD spectrum for various aromatic side chains of proteins. Adapted from (Kelly, Jess et al. 2005).

Figure 3.11 Schematic representation of an ITC instrument. The sample cell and the reference cell are kept at the same temperature. The reference cell is always kept at the experimental temperature. The ligand component of the interaction is placed in the syringe and the other in the cell. Upon the first titration (injection), the change in heat associated with binding (endothermic or exothermic) results in a change in temperature in the sample cell. A change in power (heat/s) is required to return the cells to identical temperatures $(T)(\Delta T=0)$. This change in power is recorded during injections. The binding isotherm and the integrated thermogram are shown in the top right. Adapted from (Ladbury and Chowdhry, 1996; Ladbury, 2004).

Figure 3.12 A schematic diagram illustrating isothermal parameters being generated from an isotherm fitting curve.

Figure 4.1 The aromatic clusters identified in DREAM C-terminus (grey solid surface) and N-terminus (blue solid surface). EF-hand 1 in DREAM is shown in cyan, EF-hand 2 in green, EF-hand 3 in yellow, EF-hand 
4 in orange, W169 in wireframe surface, and calcium ions shown as yellow spheres

Figure 4.2 Fluorescence emission spectra of Apo, $\mathrm{Mg}^{2+}, \mathrm{Ca}^{2+}$, and $\mathrm{Ca}^{2+} \mathrm{Mg}^{2+} \mathrm{DREAM}$ upon excitation at $295 \mathrm{~nm}$. Note that the emission spectrum of ApoDREAM and $\mathrm{Mg}^{2+}$ DREAM are almost identical, and likewise for $\mathrm{Ca}^{2+} \mathrm{DREAM}$ and $\mathrm{Ca}^{2+} \mathrm{Mg}^{2+} \mathrm{DREAM}$

Figure 4.3 Stern-Volmer plot for acrylamide quenching of DREAM in the absence/presence of $\mathrm{Ca}^{2+}$ and $\mathrm{Mg}^{2+}$. Error bar represents standard deviations of three independent measurements.....

Figure 4.4 Frequency-domain intensity decay of $40 \mu \mathrm{M}$ ApoDREAM (open squares), $\mathrm{Mg}^{2+} \mathrm{DREAM}$ (open circles), $\mathrm{Ca}^{2+} \mathrm{DREAM}$ (closed squares), and $\mathrm{Mg}^{2+} \mathrm{Ca}^{2+}$ DREAM (closed circles). The solid lines represent the data fitting using a Gaussian distribution model and single exponential decay model. Note that the experimental data for ApoDREAM and $\mathrm{Mg}^{2+}$ DREAM are nearly identical, and likewise for the data for $\mathrm{Ca}^{2+}$ DREAM and $\mathrm{Ca}^{2+} \mathrm{Mg}^{2+}$ DREAM.

Figure 4.5 Fluorescence lifetime rotational distribution of $\mathrm{Mg}^{2+} \mathrm{DREAM}$ and $\mathrm{Ca}^{2+} \mathrm{Mg}^{2+}$ DREAM using a continuous Gaussian distribution and a discrete single exponential decay component. To simplify, the lifetime rotational distribution curves of ApoDREAM and $\mathrm{Ca}^{2+}$ DREAM are not shown.

Figure 4.6 CD spectra of Apo, $\mathrm{Mg}^{2+}, \mathrm{Ca}^{2+}$, and $\mathrm{Ca}^{2+} \mathrm{Mg}^{2+}$ DREAM in the farUV region. The concentration of the protein was $40 \mu \mathrm{M}$ in $10 \mathrm{mM}$ Tris buffer (pH 7.4), $1 \mathrm{mM}$ DTT, and $10 \mathrm{mM}$ LDAO. Spectra were corrected for the baseline of the buffer....

Figure 4.7 (a): CD spectra of $40 \mu \mathrm{M}$ ApoDREAM in the presence of $0,1,2,3$, 4, 5, 6, 7, and 7.4 M GuHCl. (b): Representative equilibrium unfolding traces of DREAM as determined by the CD signal change at $220 \mathrm{~nm}$. Solid black lines are fitting curves of experimental data using equation 3.60 in section 3.2.3.4. The thermodynamic parameters are reported in Table 4.3

Figure 4.8 Root-mean-square fluctuations (RMSF) of the $\mathrm{C}_{\alpha}$ backbone in ApoDREAM and $\mathrm{Ca}^{2+}$ DREAM.

Figure 4.9 Top: Overlaid MD simulations structures of ApoDREAM (in red) and $\mathrm{Ca}^{2+}$ bound DREAM (in yellow). Alpha-helices $1-10$ in DREAM are labeled by $\alpha 1-\alpha 10$, respectively. EF-hand 1 is composed of $\alpha 2$ and $\alpha 3$, EF-hand 2 composed of $\alpha 4$ and $\alpha 5$, EF-hand 
3 composed of $\alpha 6$ and $\alpha 7$, EF-hand 4 composed of $\alpha 8$ and $\alpha 9$. The position of the W169 residue is shown with sticks. Bottom: $\mathrm{Ca}^{2+}$ triggered reorganization of the EF-hand 3 (left) and EF-hand 4 (right).

Figure 4.10 Top: charged and polar amino acid residues surrounding W169 in the structure of DREAM (PBD entry 2JUL, shown in blue) and Cterminal domain of DREAM (PBD entry 2E6W, shown in green). Bottom: Left: SASA of W169 in DREAM during $270 \mathrm{~ns}$ of the MD trajectory. Right: Partially buried (in blue) and solvent exposed (in green) orientation of W169 side chain in DREAM structure.

Figure 5.1 Binding isotherm for $\mathrm{Ca}^{2+}$ association to DREAM-C in the absence of $\mathrm{Mg}^{2+}$. Top panel represents for a raw power titration curve, bottom panel shows integration data, and the solid line presents the two sets of sites fitting curve.

Figure 5.2 The CD spectra of DREAM-C in the presence/absence of $\mathrm{Ca}^{2+}$ and/or $\mathrm{Mg}^{2+}$

Figure 5.3 The fluorescence steady-state emission spectra of intrinsic W169 residue (A) and the CD spectra (B) of DREAM-C.

Figure 5.4 Stern-Volmer plot for acrylamide quenching of DREAM-C in the absence/presence of $\mathrm{Ca}^{2+}$ and $\mathrm{Mg}^{2+}$

Figure 5.5 Frequency-domain intensity decays for W169 in the presence/absence of $\mathrm{Ca}^{2+}$ and/or $\mathrm{Mg}^{2+}$. Phase delays are solid symbols, modulation ratios are empty symbols, and the solid lines represent the calculated fitting curves.

Figure 5.6 Fluorescence steady-state emission spectra of nile red (A) and 1,8 ANS (B) bind to DREAM-C in the presence/absence of $\mathrm{Ca}^{2+}$ and/or $\mathrm{Mg}^{2+}$.

Figure 5.7 GuHCl-induced unfolding DREAM-C in the presence/absence of $\mathrm{Ca}^{2+}$ and/or $\mathrm{Mg}^{2+}$. Unfolding parameters were recovered using a twostate model.

Figure 6.1 Left: Protein-protein docking complex of $\mathrm{Ca}^{2+}$ DREAM (PDB: 2JUL, molecule 1) and PS1-CTF (PDB: 2KR6, molecule 1). DREAM (residues 76-256) contains $10 \alpha$-helices respectively labeled $\alpha 1-\alpha 10$, and four EF-hands: EF-1, EF-2, EF-3, and EF-4. Two $\mathrm{Ca}^{2+}$ ions (yellow sphere) are bound at EF-3 and EF-4 loops in DREAM. PS1CTF comprises two trans-membrane helices: HL7 and HL8, a solvent 
exposed helix HL $\beta$, and an intramembranous HL9 which is divided into two perpendicular helices. Right: Detailed presentation of the binding interfaces between $\mathrm{Ca}^{2+}$ DREAM and PS1-CTF. Amino acid residues from DREAM and PS1 are colored in green and red, respectively. Yellow dot lines represent for hydrogen bond interactions.

Figure 6.2 Titration curves for DREAM binding to HL9 (a) and HL $\beta$ (b) of PS1 CTF. Solid lines correspond to the best fit using equation 3.63 in Method section 3.5.2.

Figure 6.3 Titration curves for DREAM-C binding to HL9 of PS1-CTF in the presence and absence of $\mathrm{Ca}^{2+}$. Solid lines correspond to the best fit using equation 3.63 in Method section 3.5.2.

Figure 6.4 Sequence alignment of 66 amino acid residues in the carboxylterminus of mouse DREAM with various members of KChIPs subfamily and NCS1 protein using Clustal Omega program. The conserved hydrophobic residues at the binding interface with HL9 are highlighted in green. The conserved basic residues at positions 200 and 207 in the flexible loop of various members in KChIP subfamily are highlighted in blue. The neutral residue and the acidic residue of NCS 1 corresponding to positions 200 and 207 in flexible loop are highlighted in grey and red, respectively. Aromatic residues corresponding to $\pi$ - $\pi$ stacking interaction with PS 1 are in orange.

Figure 6.5 Titration curves for HL9 binding to $\mathrm{Ca}^{2+}$ DREAM-NCS1 (a) and $\mathrm{Ca}^{2+}$ DREAM-KChIP1 (b). Solid lines correspond to the best fit using equation 3.63 in Method section 3.5.2.

Figure 6.6 Titration curves for HL9 binding to $\mathrm{Ca}^{2+}$ DREAM-E234Q (a) and $\mathrm{Ca}^{2+}$ DREAM-E186Q (b). Solid lines correspond to the best fit using equation 3.63 in Method section 3.5.2.

Figure 6.7 Panel (a) is time-resolved anisotropy of HL9 in buffer solution (circles) and HL9 bound $\mathrm{Ca}^{2+}$ DREAM (square). Differential phase angle and modulation ratio are shown as solid and empty symbols, respectively. Panel (b) is a Job plot curve represents a 1:1 stoichiometry for molecular association of HL9 with $\mathrm{Ca}^{2+}$ DREAM.

Figure 6.8 Titration curves for HL9 binding to $\mathrm{Ca}^{2+}$ DREAM by monitoring FITC fluorescence emission change at $520 \mathrm{~nm}$ (inset figure). Solid lines correspond to the best fit using equation 3.63 in section 3.5.2. The dissociation constant was determined to be $0.6 \mu \mathrm{M}$. 
Figure 6.9 Kinetics trace for association of HL9 to $\mathrm{Ca}^{2+} \operatorname{DREAM}$ (a) and dissociation of HL9 from ApoDREAM (b) monitored by change in the fluorescence intensity at $520 \mathrm{~nm}$. The solid line represents single exponential fitting curve.

Figure 6.10 The Arrhenius plot of HL9 association with $\mathrm{Ca}^{2+}$ DREAM. $\mathrm{E}_{\mathrm{a}}$ and experimental error were determined from the mean and standard deviations of three independent measurements, respectively....

Figure 6.11 Apparent association rate constants $\left(\mathrm{k}_{\mathrm{on}(\mathrm{obs})}\right)$ were plotted against $\mathrm{Ca}^{2+}$ DREAM concentrations. The association rate constant $\left(\mathrm{k}_{\text {on }}\right)$ and the dissociation rate constant $\left(\mathrm{k}_{\text {off }}\right)$ were determined to be is about 0.6 $\mathrm{x} 10^{6} \mathrm{M}^{-1} \mathrm{~s}^{-1}$ and $1.0 \mathrm{~s}^{-1}$, respectively.

Figure 6.12 The bar plot represents titrations of $20 \mu \mathrm{M}$ of $\mathrm{Ca}^{2+} \mathrm{DREAM}$, ApoDREAM, $\mathrm{Ca}^{2+}$ DREAM-F252A, and ApoDREAM-F252A into $0.5 \mu \mathrm{M}$ PS1-HL9.

Figure 6.13 Titration curves for HL9 binding to $\mathrm{Ca}^{2+}$ DREAM in the presence of $0.3 \mathrm{M} \mathrm{NaCl}$. The Solid line corresponds to the best fit using equation 3.63 in section 3.5.2.

Figure 6.14 Titration of NS5806 into the $\mathrm{Ca}^{2+}$ DREAM-HL9 complex. The dissociation constant was determined by fitting the data using a single binding site displacement equation 3.64 in section 3.5.2.

Figure 6.15 Left: Superposed structures of $\mathrm{Ca}^{2+}$ DREAM (PDB code: 2JUL, molecule 1, cyan) and molecular dynamics simulation structure of ApoDREAM (yellow). Alpha helices $1-10$ in DREAM are labeled by $\alpha 1-\alpha 10$, respectively. The movement of $\alpha 10$ away from $\alpha 7$ is indicated by black arrows. Right: aromatic ring of F252 in ApoDREAM rotates $\sim 90$ degrees with respect to aromatic ring of F252 in $\mathrm{Ca}^{2+}$ bound DREAM. The guanidinium group of R200 in ApoDREAM points 180 degrees away with respect to that of $\mathrm{Ca}^{2+}$ DREAM.

Figure 7.1 Tryptophan (W169) emission spectra of Apo, $\mathrm{Mg}^{2+}, \mathrm{Ca}^{2+}$, and $\mathrm{Ca}^{2+} \mathrm{Mg}^{2+}$ bound DREAM-D150N (left panel) and DREAM-E186Q (right panel) upon excitation at $295 \mathrm{~nm}$. Conditions: $40 \mu \mathrm{M}$ DREAM in $20 \mathrm{mM}$ Tris $\mathrm{pH} 7.4,10 \mathrm{mM}$ LDAO, and $1 \mathrm{mM}$ DTT..

Figure 7.2 W169 emission spectra of Apo, $\mathrm{Mg}^{2+}, \mathrm{Ca}^{2+}$, and $\mathrm{Ca}^{2+} \mathrm{Mg}^{2+}$ bound DREAM-E234Q. Conditions: $40 \mu \mathrm{M}$ DREAM in $20 \mathrm{mM}$ Tris $\mathrm{pH}$ 7.4, $10 \mathrm{mM}$ LDAO, and $1 \mathrm{mM}$ DTT. 161 
Figure 7.3 Fluorescence emission spectra of 1,8-ANS (left panel) and 2,6-ANS (right panel) bound to DREAM-D150N $(40 \mu \mathrm{M})$. The excitation wavelength was $350 \mathrm{~nm}$ and $319 \mathrm{~nm}$ for 1,8-ANS and 2,6-ANS, respectively.

Figure 7.4 Fluorescence emission spectra of 1,8-ANS (left panel) and 2,6-ANS (right panel) bound to DREAM-E186Q $(40 \mu \mathrm{M})$. The excitation wavelength was $350 \mathrm{~nm}$ and $319 \mathrm{~nm}$ for 1,8-ANS and 2,6-ANS, respectively.

Figure 7.5 Fluorescence emission spectra of 1,8-ANS (left panel) and 2,6-ANS (right panel) bound to DREAM-E234Q $(40 \mu \mathrm{M})$. The excitation wavelength was $350 \mathrm{~nm}$ and $319 \mathrm{~nm}$ for 1,8-ANS and 2,6-ANS, respectively.

Figure 7.6 Frequency-domain W169 intensity decay of Apo, $\mathrm{Mg}^{2+}, \mathrm{Ca}^{2+}$, and $\mathrm{Ca}^{2+} \mathrm{Mg}^{2+}$ bound DREAM-D150N (left panel) and DREAM-E186 (right panel). The solid lines represent the data fitting using a combination of Gaussian distribution and discrete exponential decay model. The recovered parameters are shown Table 2.

Figure 7.7 Frequency-domain intensity decay of Apo, $\mathrm{Mg}^{2+}, \mathrm{Ca}^{2+}$, and $\mathrm{Ca}^{2+} \mathrm{Mg}^{2+}$ bound DREAM-E234Q $(40 \mu \mathrm{M})$. The solid lines represent the data fitting using a combination of Gaussian distribution and discrete exponential decay model

Figure 7.8 Frequency domain intensity decay of 1,8-ANS bound to DREAMD150N. Phase delay and modulation ratio are shown as solid symbols and empty symbols, respectively. Recovered intensity decay parameters are shown Table 7.2.

Figure 7.9 Frequency domain intensity decay of 1,8-ANS bound to DREAME186Q (left) and DREAM-E234Q (right). Phase delay and modulation ratio are shown as solid symbols and empty symbols, respectively. Recovered intensity decay parameters are shown Table 7.2 .

Figure 7.10 Circular dichroism spectra of $\mathrm{Apo}, \mathrm{Mg}^{2+}, \mathrm{Ca}^{2+}$, and $\mathrm{Ca}^{2+} \mathrm{Mg}^{2+}$ DREAM-D150N (left panel) and DREAM-E186Q (right panel). Conditions: $40 \mu \mathrm{M}$ DREAM in $20 \mathrm{mM}$ Tris $\mathrm{pH}$ 7.4, $10 \mathrm{mM}$ LDAO, and $1 \mathrm{mM}$ DTT.

Figure 7.11 Circular dichroism spectra of $\mathrm{Apo}, \mathrm{Mg}^{2+}, \mathrm{Ca}^{2+}$, and $\mathrm{Ca}^{2+} \mathrm{Mg}^{2+}$ DREAM-E234Q. Conditions: $40 \mu \mathrm{M}$ DREAM-E234Q in $20 \mathrm{mM}$ Tris $\mathrm{pH}$ 7.4, $10 \mathrm{mM}$ LDAO, and $1 \mathrm{mM}$ DTT. 
Figure 7.12 ITC isotherms for ApoDREAM (A) and $\mathrm{Mg}^{2+}$ DREAM (B) binding to DNA. The upper panels of each profile reflects the thermal power expressed in units of $\mu \mathrm{cal} / \mathrm{s}$. The lower panel shows integrated reaction heats $(\Delta \mathrm{H})$ expressed in units of $\mathrm{kcal} / \mathrm{mol}$. The solid lines present the best fitting curve using a two-set of sites model with deletion of the first titration point. The experiment was carried out by titration of $500 \mu \mathrm{M}$ of DREAM protein (in syringe) into $15 \mu \mathrm{M}$ DNA (in sample chamber) at $25^{\circ} \mathrm{C}$.

Figure 7.13 ITC isotherms for ApoDREAM-D150N (A) and ApoDREAME186Q (B), and ApoDREAM-E234Q binding to DNA. The upper panels of each profile reflects the thermal power expressed in units of $\mu \mathrm{cal} / \mathrm{s}$. The lower panel shows integrated reaction heats $(\Delta \mathrm{H})$ expressed in units of $\mathrm{kcal} / \mathrm{mol}$. The solid lines present the best fitting curve using a two-set of sites model with deletion of the first titration point. The recovered isothermal parameters are shown in Table 7.3.

Figure 7.14 ITC isotherms for $\mathrm{Ca}^{2+} \mathrm{DREAM}(\mathrm{A}), \mathrm{Ca}^{2+} \mathrm{DREAM}-\mathrm{D} 150 \mathrm{~N}$ (B), $\mathrm{Ca}^{2+}$ DREAM-E186Q (C), and $\mathrm{Ca}^{2+}$ DREAM-E234Q (D) binding to DNA. The upper panels of each profile reflects the thermal power expressed in units of $\mu \mathrm{cal} / \mathrm{s}$. The lower panel shows integrated reaction heats $(\Delta \mathrm{H})$ expressed in units of $\mathrm{kcal} / \mathrm{mol}$. The solid lines present the best fitting curve using a sequential binding sites model with deletion of the first titration point. The recovered isothermal parameters are shown in Table 7.3.

Figure 7.15 A: Time-resolved anisotropy of DNA in buffer solution (triangle), $\mathrm{Ca}^{2+}$ DREAM-DNA complex (square), and ApoDREAM-DNA complex (circle). B: Time-resolved anisotropy of DNA in buffer solution containing $100 \mu \mathrm{M} \mathrm{MgCl}$ (pentagon) and $\mathrm{Mg}^{2+}$ DREAMDNA complex (hexagon). Differential phase angle and modulation ratio are shown as solid and empty symbols, respectively. Solid lines represent the non-linear least square fit and recovered parameters are shown in Table 7.4

Figure 7.16 The backbone root-mean-square deviations (RMSD) of Apo, $\mathrm{Mg}^{2+}$, and $\mathrm{Ca}^{2+}$ DREAM during a $100 \mathrm{~ns}$ simulation time.

Figure 7.17 The root-mean-square fluctuation (RMSF) plot of $\mathrm{C}_{\alpha}$ atoms represents the fluctuations of individual residues with respect to the average structure in the structures of Apo, $\mathrm{Mg}^{2+}$, and $\mathrm{Ca}^{2+}$ bound forms of DREAM from 20 to $100 \mathrm{~ns}$ simulation time. 
Figure 7.18 Graphic representation of the monomer structures averaged over 100 ns of MD simulations of Apo (green), $\mathrm{Ca}^{2+}$ bound at EF-hands 3 and 4 (blue), $\mathrm{Ca}^{2+}$ bound at EF-hand 4 in DREAM-E186Q mutant (light blue), and $\mathrm{Ca} 2+$ bound at EF-hand 3 in DREAM-E234Q mutant (pink). All nodes and edges of the communities are colored and shown as ball and stick, respectively.

Figure 7.19 Top: The path connecting residues E186 and R98 in ApoDREAM (left), $\mathrm{Ca}^{2+}$ DREAM-E234Q mutant (right), and $\mathrm{Ca}^{2+}$ DREAM wildtype (bottom). The nodes in communities are shown as balls. Communities are colored using the same color codes as shown in Figure 7.18. The hydrophobic residues in the path connecting from R98 to E186 in $\mathrm{Ca}^{2+}$ DREAM wild-type are shown in stick (bottom).

Figure 7.20 The path connecting residues R98 and E234 in ApoDREAM (green), $\mathrm{Ca}^{2+}$ DREAM (blue). The nodes in communities are shown as balls. Communities are colored using the same color scheme as shown in Figure 7.18. The hydrophobic residues within paths connecting from R98 to E234 in $\mathrm{Ca}^{2+}$ DREAM are shown in stick. Note that only two hydrophobic residues $\mathrm{I} 157$ and L167 surrounding W169 were observed in the path.

Figure 7.21 The MD simulation model of $\mathrm{Mg}^{2+} \mathrm{DREAM}$ : Association of $\mathrm{Mg}^{2+}$ at EF-hand 2 forms a new hydrophobic cluster (shown as pink solid surface), that has not been observed in either Apo or $\mathrm{Ca}^{2+}$ DREAM. The other two aromatic clusters identified in the C-terminus and $\mathrm{N}$ terminus are conserved in the NMR structure of $\mathrm{Ca}^{2+}$ DREAM and shown as grey solid surface and blue solid surface.

Figure 7.22 Left: Dynamical network analysis of the 100ns MD simulation of the first $6 \alpha$-helices in $\mathrm{Mg}^{2+}$ DREAM $\mathrm{N}$-terminus displayed in communities (each community has different color). Right: The dynamical network Hydrophobic cluster L93, L96, and F100 on the inner side of $\alpha 2$ contact with the hydrophobic residues F121 (from a3), F148, F151, V151, L155, and L159 from $\alpha 5$ (exiting helix of EFhand 2) that is stabilized upon $\mathrm{Mg}^{2+}$ association to DREAM. The basic and polar residues K90, K91, Q94, S95, R98, and K101 interfacing with DNA are shown on the outer site of $\alpha 2$. The leucine residues L155 and L159 at the dimeric interface of DREAM are in red, whereas the basic residues forming a salt bridge between $\alpha 2$ and $\alpha 5$ are shown in light blue.

Figure 7.23 Left: Dynamical network analysis of the 100ns MD simulation of the first $6 \alpha$-helices in ApoDREAM N-terminus displayed in communities (each community colored differently). Right: In the 
absence of $\mathrm{Mg}^{2+}$, the $\alpha 2$ is not involved in an extended community formed by $\alpha 1, \alpha 5$, and $\alpha 6$, suggesting a decrease in stability of the $\mathrm{N}$ terminus domain despite of the salt bridge formation between residues E92 and R160 (in light blue, bottom).

Figure 7.24 Dynamical network analysis of the 100ns MD simulation of the first $6 \alpha$-helices in $\mathrm{Ca}^{2+}$ DREAM N-terminus displayed in communities (each community colored differently). Rearrangement of $\alpha 1$ partially blocks the DNA binding site on $\alpha 2$.

Figure 7.25 (a): The MD simulation of docking complex between ApoDREAM (residues 75-256) and 25 oligomers corresponding to the DRE sequence of prodynorphin gene (namely DNA). (b): A closer view at the interaction interface between ApoDREAM and DNA. Yellow dot lines represent hydrogen bond interactions. (c): the sequence of 25 oligonucleotides corresponding to the DRE. (d): the sequence alignment of 45 amino acid residues in the N-terminus of DREAM (residues: 75-120, top) and that of the non-DNA binding recoverin (residues: 12-57) to highlight the positively charged residues in DREAM participating to the interaction with DNA.

Figure 7.26 Left: Residue W169 is buried in a more hydrophobic environment observed in ApoDREAM (yellow) compared to that obtained in ApoDREAM-D150N mutant (green). Right: The superposition of five $\alpha$-helices in the N-terminal domain of ApoDREAM (yellow) and ApoDREAM-D150N (green).

Figure 7.27 Top: Amino acid sequence alignment of residues (K90 - N102) in $\alpha 2$ of ApoDREAM (green) and $\mathrm{Ca}^{2+}$ DREAM (blue). Bottom: The dimer $\mathrm{Ca}^{2+}$ DREAM was constructed according to the model proposed by Lusin et at. (Lusin et al., 2008).

Figure 8.1 The schematic presentation of AOT molecule (left) and a reverse micelle particle containing an $\mathrm{Mb}$ molecule encapsulated within internal water pool (right).

Figure 8.2 Optical absorption spectra of $\mathrm{Mb}$ (left) and MP-11 (right) encapsulated within $\mathrm{RM} w=40$. The traces for $\mathrm{Fe}^{3+} \mathrm{Mb} / \mathrm{Fe}^{3+} \mathrm{Mp}-11$ are shown as solid thick lines, for $\mathrm{Fe}^{2+} \mathrm{Mb} / \mathrm{Fe}^{2+} \mathrm{MP}-11$ as dashed lines and for $\mathrm{CO}-\mathrm{Mb}$ and $\mathrm{CO}-\mathrm{MP} 11$ as solid thin lines. .205

Figure 8.3 PAC traces for $\mathrm{CO}$ photo-dissociation from $\mathrm{Fe}^{2+} \mathrm{Mb}$ in AOT RM of $\mathrm{w}_{\mathrm{o}}=40$ (thick black line), for the reference compound $\left(\mathrm{Fe}^{3+} \mathrm{Mb}\right.$ in AOT RM of $w_{0}=40$ ) and the fit (gray line). 
Figure 8.4 Transient absorption trace for $\mathrm{CO}$ rebinding to $\mathrm{Fe}^{2+} \mathrm{Mb}$ encapsulated within $\mathrm{RM}, \mathrm{w}_{\mathrm{o}}=40$. Inset: Arrhenius plot of the rate constants for the fast (circles) and slow (squares) phase of the $\mathrm{CO}$ rebinding to $\mathrm{Mb}$... 


\section{LIST OF ABBREVIATIONS}

\begin{tabular}{|c|c|}
\hline ABBREVIATION & FULL NAME \\
\hline $\mathrm{Ca}^{2+}$ & Calcium ion \\
\hline $\mathrm{CD}$ & Circular dichroism \\
\hline CTF & Carboxy-terminal fragment \\
\hline DNA & $\begin{array}{l}\text { Downstream regulatory elements sequence ( } 5^{\prime} \text { - } \\
\text { GAAGCCGGAGTCAAGGAGGCCCCTG-3') of } \\
\text { prodynorphin gene }\end{array}$ \\
\hline DMSO & Dimethyl sulfoxide \\
\hline DREAM & Downstream regulatory element antagonist modulator \\
\hline DREAM-C & DREAM C-terminal domain \\
\hline DREAM-D150N & DREAM mutant with an inactive EF-hand 2 \\
\hline DREAM-E186Q & DREAM mutant with an inactive EF-hand 3 \\
\hline DREAM-E234Q & DREAM mutant with an inactive EF-hand 4 \\
\hline DTT & Dithiothreitol \\
\hline EGTA & 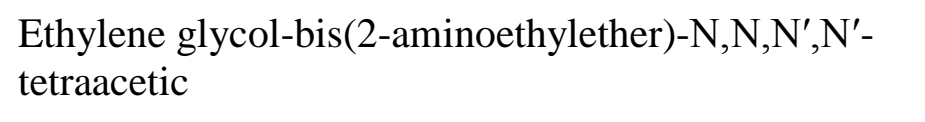 \\
\hline $\mathrm{EtBr}$ & Ethidium bromide \\
\hline EDTA & $\begin{array}{l}\text { 2-(\{2-[Bis(carboxymethyl)amino]ethyl }\} \\
\text { (carboxymethyl)amino)acetic acid }\end{array}$ \\
\hline EF-hand & $\mathrm{Ca}^{2+}$ binding helix-loop-helix motifs \\
\hline FITC & Fluorescein isothiocyanate \\
\hline $\mathrm{GuHCl}$ & Guanidine hydrochloride \\
\hline IPTG & Isopropyl $\beta$-D-1-thiogalactopyranoside \\
\hline
\end{tabular}




\begin{tabular}{|c|c|}
\hline ITC & Isothermal titration calorimetry \\
\hline $\mathrm{K}_{\mathrm{a}}$ & Association constant \\
\hline $\mathrm{K}_{\mathrm{d}}$ & Dissociation constant \\
\hline $\mathrm{K}_{\mathrm{i}}$ & Inhibition constant \\
\hline $\mathrm{Mg}^{2+}$ & Magnesium ion \\
\hline NLLS & Non-linear least squares \\
\hline NS5806 & $\begin{array}{l}\mathrm{N} \text {-[3,5-Bis(trifluoromethyl)phenyl]-N'-[2,4-dibromo-6- } \\
\text { (2H-tetrazol-5-yl)phenyl]urea }\end{array}$ \\
\hline NTF & Amino-terminal fragment \\
\hline NCS & Neuronal calcium sensor \\
\hline PAC & Photoacoustic calorimetry \\
\hline PS1 & presenilin 1 protein \\
\hline KChIP & Potassium channel interaction protein \\
\hline RMSD & Root mean square deviation \\
\hline RMSF & Root mean square fluctuation \\
\hline SDS & Sodium dodecyl sulfate \\
\hline SASA & Solvent accessible surface area \\
\hline 1,8 -ANS & 1-anilinonaphthalene-8-sulfonic acid \\
\hline TRIS & 2-Amino-2-hydroxymethyl-propane-1,3-diol \\
\hline 1,5-IAEDANS & $\begin{array}{l}\text { (5-((((2-Iodoacetyl)amino)ethyl)amino)Naphthalene-1- } \\
\text { Sulfonic Acid) }\end{array}$ \\
\hline$\alpha$ & alpha-helix \\
\hline$\Delta \mathrm{H}$ & Enthalpy change \\
\hline$\Phi$ & Quantum yield \\
\hline
\end{tabular}




\section{PREFACE}

This dissertation consists of the research studies carried out from January 2011 to June 2016 by Khoa N. Pham. During this time, some of these results have been published in peer-reviewed journals, particularly Chapter 4 (Pham and Miksovska, 2015), Chapter 6 (Pham and Miksovska, 2016), Chapter 8 (Larsen, Vetromile, Pham, Miksovska, 2011). The results in Chapter 5 were described in a manuscript that will be submitted by Pham, Gonzalez, and Miksovska. Data represented in Chapter 7 were described in a manuscript that will be submitted to the Biochemistry journal by Pham and Miksovska. 


\section{INTRODUCTION}

\subsection{Calcium and intracellular $\mathrm{Ca}^{2+}$ signaling network}

Calcium plays an important role in many biological processes. In the solid form, calcium has a structurally supportive function (Bronner et al., 1992; Vogel et al., 2002). Soluble calcium ions $\left(\mathrm{Ca}^{2+}\right)$ act as an intracellular signaling ion that interacts with calcium binding proteins to mediate numerous physiological functions, including fertilization, contraction, metabolism, cell cycle progression, signal transduction, replication, gene expression, and electrochemical responses (Carafoli et al., 2007; Gifford et al., 2007; Berridge et al., 1997). In the cytoplasm, intracellular $\mathrm{Ca}^{2+}$ concentration at the resting level is about $100 \mathrm{nM}$, while the extracellular $\mathrm{Ca}^{2+}$ concentration is about $2 \mathrm{mM}$, creating a gradient between the intracellular and extracellular $\mathrm{Ca}^{2+}$ concentrations (Vogel et al., 1994; Clapham, 2007). Such low cytoplasmic $\mathrm{Ca}^{2+}$ concentration is well maintained by ATPdependent $\mathrm{Ca}^{2+}$ pumps, which pump $\mathrm{Ca}^{2+}$ out of the cell or into specialized organelles such as the sarcoplasmic reticulum in muscle cells. Intracellular $\mathrm{Ca}^{2+}$ mediates its effects and signaling by association to numerous proteins including membrane binding proteins and cytoplasmic proteins. Subsequently, the $\mathrm{Ca}^{2+}$ signal is transduced into biological functions by triggering different intracellular pathways (Carafoli, 2007; Clapham, 2007).

The regulation of a single $\mathrm{Ca}^{2+}$ ion in numerous cellular processes remarkably highlights its versatility in cellular functions (Carafoli et al., 2001; Berridge, 2001). The versatility of $\mathrm{Ca}^{2+}$ signaling has been attributed to its speed, amplitude, and wide range of spatial and temporal signals (Berridge, 2001). Each cell type has distinctive components to create various $\mathrm{Ca}^{2+}$ signaling systems with different spatial and temporal dynamics (Berridge et al., 2003). A common $\mathrm{Ca}^{2+}$ signaling network consists of four functional 
components with the $\mathrm{Ca}^{2+}$ transients being controlled by the ON/OFF reactions outlined in Figure 1.1 (Berridge, 1997; Berridge, 2001; Berridge et al., 2003).

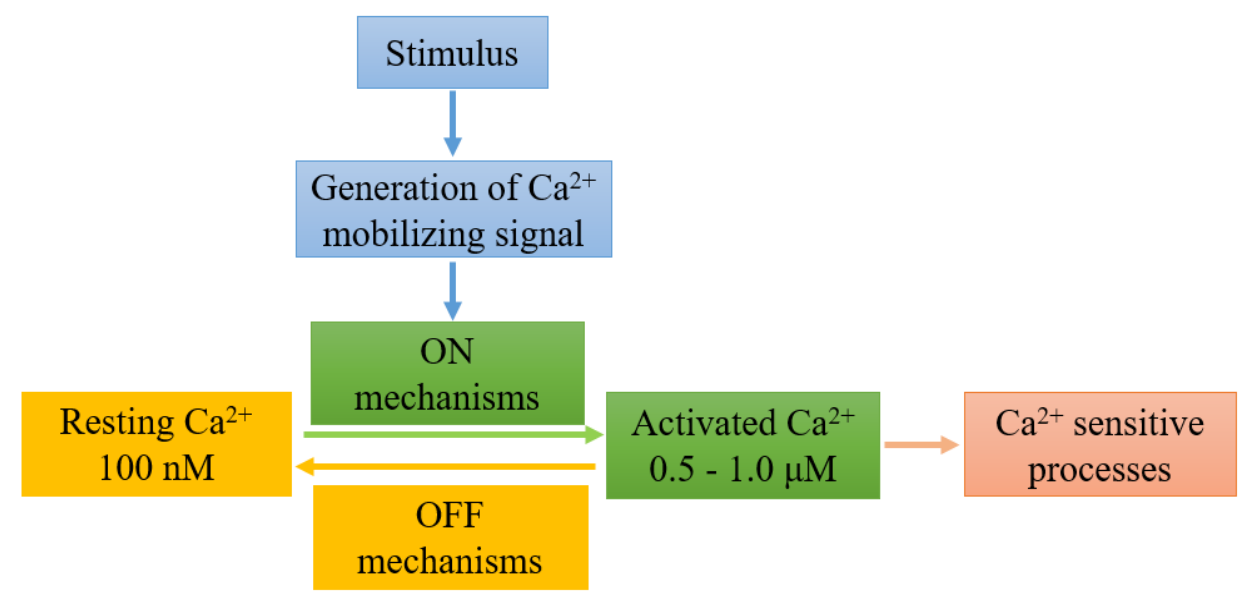

Figure 1.1: Scheme illustrating a common intracellular $\mathrm{Ca}^{2+}$ signaling network. Adapted from (Berridge et al., 2000).

The $\mathrm{Ca}^{2+}$ signaling network is initiated by the entry of $\mathrm{Ca}^{2+}$ into the cell. During the ON mechanism, the stimulus depolarizes a cell membrane causing a large electrochemical gradient across the cell membrane to activate various $\mathrm{Ca}^{2+}$ entry channels, such as voltageoperated channels (VOCs), which allow $\mathrm{Ca}^{2+}$ entry from extracellular medium (Berridge et al., 2003). In addition, $\mathrm{Ca}^{2+}$ can also be released into the cytoplasm from internal storage held within the membranes of the endoplasmic reticulum (ER) and the sarcoplasmic reticulum (SR). The process is tightly controlled by various channels such as inositol 1,4,5triphosphate receptor (InsP3R) and ryanodine receptor (RYR) (Berridge, 2001). The $\mathrm{Ca}^{2+}$ that flows into the cytoplasm during the ON mechanism does not remain free (Gifford et al., 2007). Instead, most of the cytoplasmic $\mathrm{Ca}^{2+}$ is bound to calcium buffer proteins, while only a small portion of free $\mathrm{Ca}^{2+}$ binds to calcium effectors such as calmodulin $(\mathrm{CaM})$, troponin, and neuronal $\mathrm{Ca}^{2+}$ sensor proteins in the brain. These $\mathrm{Ca}^{2+}$ effectors activate 
various $\mathrm{Ca}^{2+}$ sensitive processes that translate $\mathrm{Ca}^{2+}$ signals into cellular responses by triggering many different signaling pathways (Berridge, 2001). Finally, when the $\mathrm{Ca}^{2+}$ signal has carried out its functions, it is rapidly removed from the cytoplasm by various pumps and exchangers via the OFF mechanism (Berridge, 2001; Berridge et al., 2003).

\subsection{Intracellular calcium binding proteins}

Intracellular $\mathrm{Ca}^{2+}$ binding proteins (CaBPs) are classified into two classes depending on their functions: $\mathrm{Ca}^{2+}$ buffer proteins and $\mathrm{Ca}^{2+}$ sensor proteins (Gifford et al., 2007). $\mathrm{Ca}^{2+}$ buffer proteins such as calbindin and parvalbumin, which are found in many cell types of various organs, bind $\mathrm{Ca}^{2+}$ with high affinity but do not undergo any significant conformational changes (Skelton et al., 1994; Ikura, 1996). The main function of $\mathrm{Ca}^{2+}$ buffer proteins is to modulate the $\mathrm{Ca}^{2+}$ signals as they load $\mathrm{Ca}^{2+}$ during the $\mathrm{ON}$ mechanism and unload $\mathrm{Ca}^{2+}$ during the OFF mechanism (Gifford et al., 2007). Another important function of $\mathrm{Ca}^{2+}$ buffers is to control $\mathrm{Ca}^{2+}$ signals transmitted throughout the cell as they limit both the amplitude and duration of $\mathrm{Ca}^{2+}$ signal (Braunewell et al., 1999). In contrast, $\mathrm{Ca}^{2+}$ sensor proteins bind to $\mathrm{Ca}^{2+}$ and undergo significant conformational changes, which allows them to interact with diverse proteins and biomacromolecules to regulate various $\mathrm{Ca}^{2+}$ sensitive processes (Braunewell et al., 1999; Burgoyne et al., 2001). Subsequently, $\mathrm{Ca}^{2+}$ sensor proteins frequently serve as effector or modulator proteins to transduce $\mathrm{Ca}^{2+}$ signals into appropriate physiological responses (Braunewell et al., 1999). Calcium binding proteins have been shown to be involved in several aspects of $\mathrm{Ca}^{2+}$ regulation in many cell types including neurons (Burgoyne et al., 2001). Since the objectives of this dissertation are focused on the $\mathrm{Ca}^{2+}$ signal transduction and target interactions of the downstream regulatory element antagonist modulator (DREAM) protein, which is a particular member 
of neuronal $\mathrm{Ca}^{2+}$ sensor (NCS) proteins, general aspects of NCS proteins, including their structure-function relationships, will be briefly discussed in the next section.

\subsection{Neuronal calcium sensor (NCS) proteins and the biological functions of $\mathrm{Ca}^{2+}$ binding to NCS proteins}

The NCS protein family constitutes the largest class of intracellular calcium binding proteins found in many organisms ranging from yeast to humans. These NCS proteins are characterized by their remarkable ability to bind and release $\mathrm{Ca}^{2+}$ over physiological ranges of intracellular $\mathrm{Ca}^{2+}$ concentrations and to undergo conformational changes (Braunewell et al., 1999; Burgoyne et al., 2001). Subsequently, these effectors interact with and regulate various activities of the targeted proteins. All NCS proteins contain four calcium binding helix-loop-helix motifs (namely EF-hands) (Burgoyne et al., 2001). The conserved sequence $\mathrm{CPxG}$ ( $\mathrm{x}$ is any residue) in the $\mathrm{Ca}^{2+}$ binding loop of the first EF-hand prevents EF-hand 1 from binding to $\mathrm{Ca}^{2+}$ or $\mathrm{Mg}^{2+}$, an abundant intracellular divalent cation with very similar chemical properties to $\mathrm{Ca}^{2+}$ (Romani and Scarpa, 1992; Gifford et al., 2007). On the other hand, EF-hands 2, 3, and 4 are able to bind $\mathrm{Ca}^{2+}$ with micromolar or submicromolar affinity (Braunewell et al., 1999; Burgoyne et al., 2001; Ames et al., 2012; Burgoyne et al., 2015). The NCS proteins are primarily expressed in photoreceptor cells and neurons. They control almost all aspects of neuronal functions including regulation of neurotransmission, short-term synaptic plasticity, regulation of $\mathrm{Ca}^{2+}$ channel, neuronal growth, learning (neuronal calcium sensor protein 1), anti-apoptosis (hippocalcin protein), guanylyl cyclase activation and recycling (neurocalcin- $\delta$, visinin-like proteins 1 and 2 ), light adaptation (recoverin), regulation of retinal guanylyl cyclase (guanylate cyclaseactivating proteins 1,2 , and 3 ), regulation of $\mathrm{Kv} 4.3$ and $\mathrm{Kv} 1.5$ potassium channels 
(potassium channel interacting proteins (KChIP) 1, 2, 3, and 4), regulation of presenilin processing (KChIP 3 and 4), and repression of gene transcription (KChIP 3) (Pongs et al., 1993; Burgoyne et al., 2001; Burgoyne et al., 2004; Burgoyne et al., 2007).

The functions of NCS proteins are diverse as they control numerous neuronal events in $\mathrm{Ca}^{2+}$ signaling pathways. The diversity has been attributed to distinct types of $\mathrm{Ca}^{2+}$ signals that differ in magnitude, duration, and localization (Burgoyne et al., 2001; Burgoyne et al., 2007). As a consequence of these actions of various NCS proteins, the $\mathrm{Ca}^{2+}$ signal is transduced into specific changes in cellular function. In addition, these distinct outcomes of the $\mathrm{Ca}^{2+}$ signal directly depend on the ability of $\mathrm{Ca}^{2+}$ sensing of different proteins in the NCS family, the localization of NCS proteins relative to the $\mathrm{Ca}^{2+}$ signal, and interactions of NCS proteins with specific targeted proteins (Burgoyne, 2007).

The ability of $\mathrm{Ca}^{2+}$ sensing, commonly known as $\mathrm{Ca}^{2+}$ binding affinity, varies in different proteins of the NCS family. The adjustment to $\mathrm{Ca}^{2+}$ affinity of individual proteins depends on the variation in the sequence of each EF-hand (Burgoyne, 2007). All members of the NCS family possess four canonical EF-hands, each of which is composed of a nineresidue entering $\alpha$-helix, a nine-residue $\mathrm{Ca}^{2+}$ binding loop, and an eleven-residue exiting $\alpha$-helix. The entering helix is oriented almost at a right angle to the exiting helix when $\mathrm{Ca}^{2+}$ is bound (Figure 1.2) (Gifford et al., 2007).

Most frequently, the $\mathrm{Ca}^{2+}$ ion is coordinated by seven ligands that are provided by oxygen atoms in the side chain of residues in the EF-hand $\mathrm{Ca}^{2+}$ binding loop (namely EFhand loop) and in the peptide backbone. In some proteins, the oxygen atom is provided by a water molecule stabilized by a hydrogen bond to one residue in the loop (Figure 1.2) (Gifford et al., 2007). 


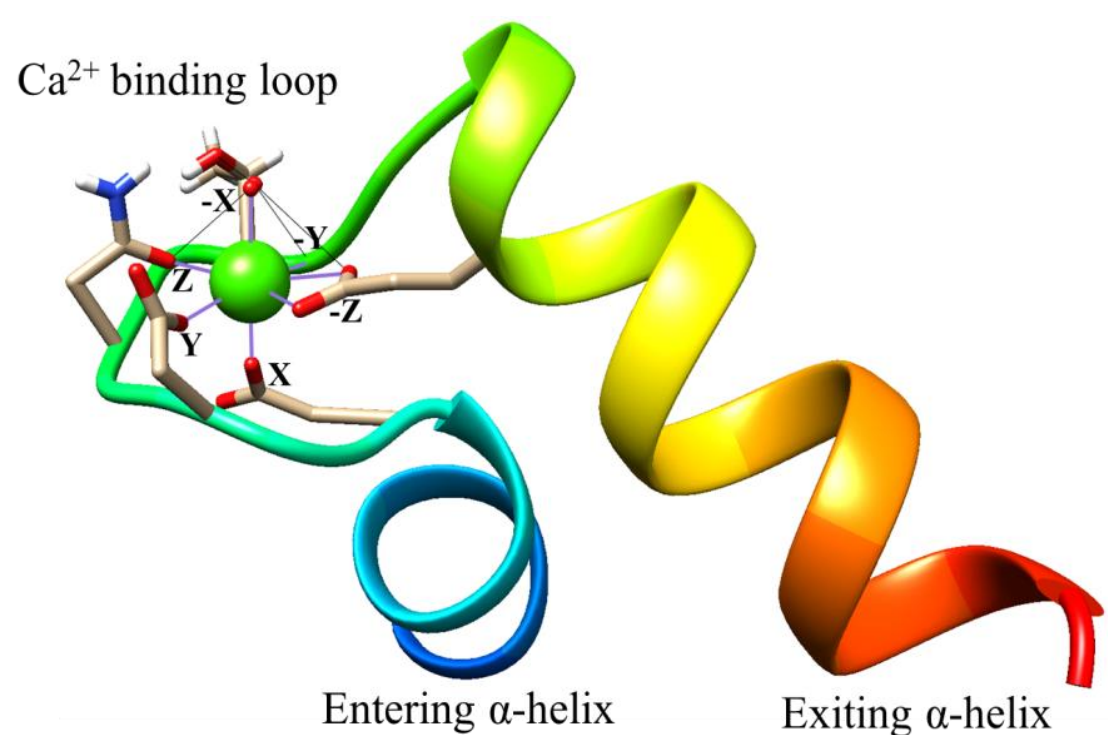

Figure 1.2: A single EF-hand 2 of recoverin (PDB code: 4YI8). The EF-hand consists of an entering $\alpha$-helix, a $\mathrm{Ca}^{2+}$ binding loop, and an exiting $\alpha$-helix. The $\mathrm{Ca}^{2+}$ atom (green sphere) is coordinated by a canonical $\mathrm{Ca}^{2+}$ binding loop illustrating the pentagonal bipyramidal coordination of the $\mathrm{Ca}^{2+}$ ion (purple lines) and the hydrogen bonding found in the loop (black lines). The side chain oxygen atoms are in red and the coordinating oxygen atoms from water are shown as red spheres.

Amino acid residues that bind to $\mathrm{Ca}^{2+}$ typically contain an oxygen atom in the side chain, such as asparagine, glutamine, aspartic acid, and glutamic acid (Falke et al., 1994; Gifford et al., 2007). Indeed, $\mathrm{Ca}^{2+}$ ligands are more frequently provided by the side chain of an aspartic acid residue, which reflects the preference of the EF-hand loop for ligands of the less bulky side chain (McPhalen et al., 1991; Gifford et al., 2007). The geometry of a canonical EF-hand is a pentagonal bipyramid, in which five of the seven $\mathrm{Ca}^{2+}$ ligands are provided by side chains of amino acid residues in the $\mathrm{Ca}^{2+}$ binding loop, and the other two ligands are supplied by the side chain of a glutamic acid residue located at the twelfth position of the $\mathrm{Ca}^{2+}$ binding loop (Figure 1.3) (Gifford et al., 2007) . 
Canonical $\mathrm{Ca}^{2+}$ binding loop

\begin{tabular}{|c|c|c|c|c|c|c|c|c|c|c|c|c|}
\hline Residue position & 1 & 2 & 3 & 4 & 5 & 6 & 7 & 8 & 9 & 10 & 11 & 12 \\
\hline Coordinate ligand & $+\mathrm{X}$ & & $+\mathrm{Y}$ & & $+Z$ & & $-\mathrm{Y}$ & & $-X$ & & & $-Z$ \\
\hline Most common & Asp & Lys & Asp & Gly & Asp & Gly & Thr & Ile & Asp & Phe & Glu & Glu \\
\hline Occurrence (\%) & 100 & 29 & 76 & 56 & 52 & 96 & 23 & 68 & 32 & 23 & 29 & 92 \\
\hline Frequently & & Ala & Asn & Lys & Ser & Phe & Val & Ser & Tyr & Asp & Asp & \\
\hline observed & & Gln & & Arg & Asn & & Lys & Leu & Thr & Ala & Lys & \\
\hline & & Thr & & Asn & & & Gln & & Glu & Thr & Ala & \\
\hline & & Val & & & & & Tyr & & Asn & Leu & Pro & \\
\hline & & Ile & & & & & Glu & & Gly & Glu & Asn & \\
\hline & & Ser & & & & & Arg & & Gln & Lys & & \\
\hline
\end{tabular}

Figure 1.3: The sequence of a canonical $\mathrm{Ca}^{2+}$ binding loop. $\mathrm{The}^{2+2+}$ ligands are indicated by their positions in the loop. The ninth $\mathrm{Ca}^{2+}$ ligand (-X coordinate) is typically provided by a water molecule. Adapted from reference (Gifford et al., 2007).

In addition, the residues in the $\mathrm{Ca}^{2+}$ binding loop are aligned on the pentagonal bipyramid axes in a manner that the oxygen atoms in the side chain of residues at positions $3,5,7$, and 12 align along the vertices $+\mathrm{Y},+\mathrm{Z},-\mathrm{Y}$, and $-\mathrm{Z}$ of the planar pentagon, respectively. Oxygen atoms in the side chain of residues at positions 1 and 9 chelate with $+\mathrm{X}$ and $-\mathrm{X}$ axes respectively on the axial position, which is perpendicular to the $\mathrm{Y} / \mathrm{Z}$ plane as shown in Figure 1.2 (Strynadka et al., 1989; Gifford et al., 2007).

The four EF-hands in NCS proteins always occur in pairs, which form two discrete domains: one is at the $\mathrm{N}$-terminus, and the other is at the $\mathrm{C}$-terminus (Figure 1.4) (Burgoyne et al., 2001). The pairing of EF-hands in NCS and other EF-hand proteins often displays the positive cooperativity for $\mathrm{Ca}^{2+}$ binding, which minimizes the required $\mathrm{Ca}^{2+}$ signal to reach protein saturation, regulating their target proteins (Gifford et al., 2007). 


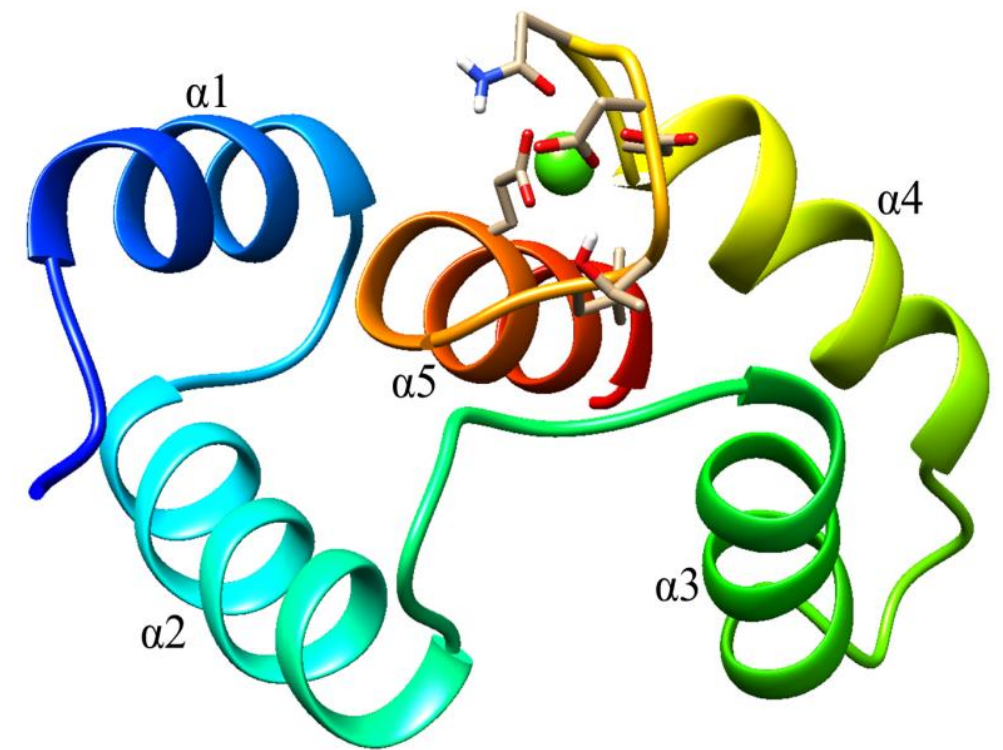

Figure 1.4: N-terminal domain of recoverin contains a pair of EF-hands (PDB code: 4YI8, residues: 1-94). Alpha helices, denoted by $\alpha, 1$ to 5 are respectively labeled. EF-hand 1 is composed of $\alpha 2$ and $\alpha 3$; likewise EF-hand 2 contains $\alpha 4$ and $\alpha 5 . \mathrm{Ca}^{2+}$ is shown as a green sphere.

As previously mentioned, the diverse functions of NCS proteins in neuronal $\mathrm{Ca}^{2+}$ signaling pathways are related to the differences of structural properties of NCS proteins such as the number of bound $\mathrm{Ca}^{2+}$ in the proteins and $\mathrm{Ca}^{2+}$ binding affinity (Burgoyne et al., 2001; Burgoyne, 2007). The molecular details of the NCS proteins' structures have been extensively reviewed in literatures (Braunewell et al., 1999; Burgoyne et al., 2001; Burgoyne et al., 2004; Burgoyne, 2007; Haynes et al., 2008; McCue et al., 2010; Burgoyne et al., 2012; Ames et al., 2012; Burgoyne et al., 2015). Here, I highlight the differences in the structure among proteins in the NCS family to exemplify the structure-function relationship of these proteins in $\mathrm{Ca}^{2+}$ signal pathways.

According to Burgoyne et al., NCS proteins are classified into five classes on the basis of their available sequences in the human genome (Figure 1.5) (Burgoyne et al., 2001). In this context, class A contains only a single gene of frequenin protein, which is 
also known as neuronal $\mathrm{Ca}^{2+}$ sensor protein 1 (NCS 1). In contrast, class B is comprised of three members of visinin-like proteins (VILIP 1,2 , and 3), hippocalcin, and neurocalcin- $\delta$ protein. Class $\mathrm{C}$ is the second smallest class in the NCS family and contains a single recoverin protein. Class D is composed of guanylate cyclase activating proteins (GCAPs) 1, 2, and 3. Class $\mathrm{E}$ is comprised of four potassium channel interacting proteins (KChIPs) 1, 2, 3, and 4 (Burgoyne et al., 2001).

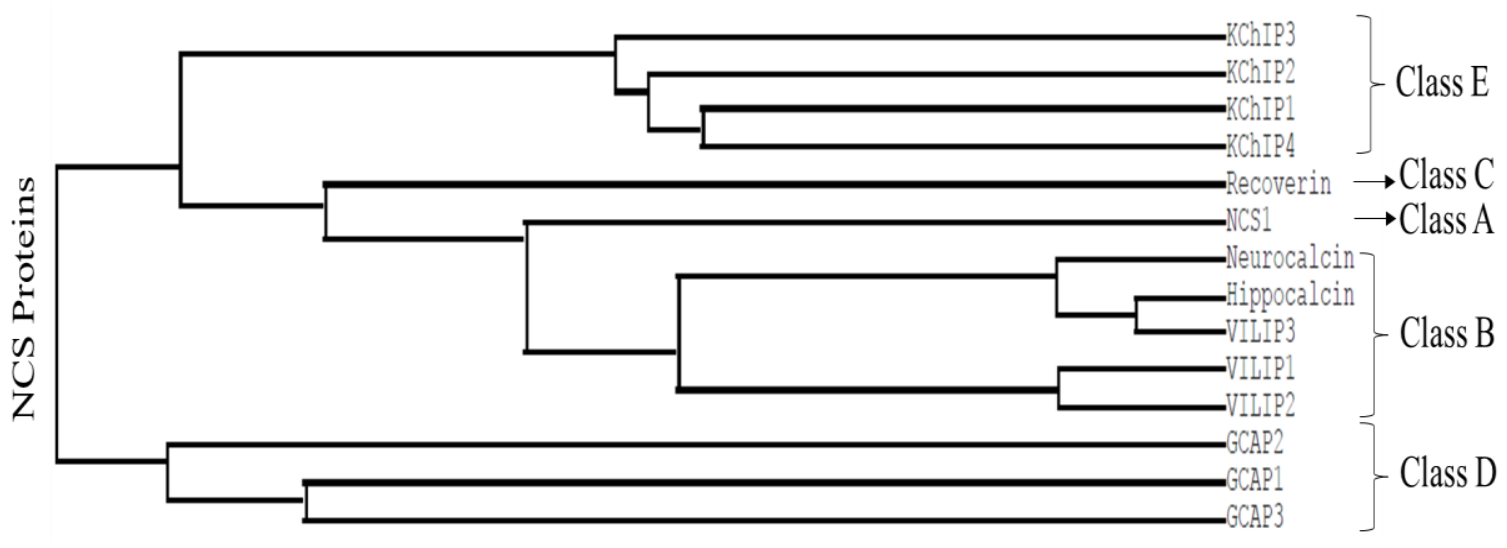

Figure 1.5: Representation of phylogenetic relationships in NCS proteins. Adapted from (Burgoyne et al., 2001).

Structurally, all members of the NCS family are small proteins containing between 190 and 270 amino acid residues, which are arranged into eight to twelve $\alpha$-helices. Amino acid sequences of NCS proteins are highly similar, particularly in the regions comprised of $\mathrm{Ca}^{2+}$ binding loops (Figure 1.6). In addition, the structures of NCS proteins are heterogeneous in $\mathrm{Ca}^{2+}$ free forms, but become more compact when $\mathrm{Ca}^{2+}$ is bound (Burgoyne et al., 2001). 


NCS1
GCAP1
GCAP2
GCAP3
Hippocalcin
Recoverin
VILIP1
VILIP2
VILIP3
KChIP1
KChIP2
KChIP3
KChIP4
NCS1
GCAP1
GCAP2
GCAP3
Hippocalcin
Recoverin
VILIP1
VILIP2
VILIP3
KChIP1
KChIP2
KChIP3
KChIP4

NCS1
GCAP1
GCAP2
GCAP3
Hippocalcin
Recoverin
VILIP1
VILIP2
VILIP3
KChIP1
KChIP2
KChIP3
KChIP4

NCS1

GCAP

GCAP

GCAP3

Hippoc

VILIP1

Recoverin
VILIP2

VILIP3

KChIPI

KChIP2

KChIP3

KChIP4

NCS1

GCAP

GCAP2

GCAP3

Hippocalci

Recoverin

VILIP1

VILIP2

VILIP

KChIP

KChIP3

KChIP2

\section{EF-2 $\mathrm{Ca}^{2+}$ binding loo}

KGFIKDCPSGQLDAAGFOKIYKOFFPFGDPTKFATFVENVFD ENKDGRTEFSEFIQALSV 91

KKFMTECPSGQLTLYEFRQFFGLKNLSPSASQYVEOMFETFD FNKDGYIDFMEYVAALSL 82

KKFVMECPSGTLFMHEFKRFFKVTD-DEEASQYVEGMFRAFDKNGDNTIDELEYVAALNL 84

RTFMMEYPSGLOTLHEFKTLLGLOGLNOKANKHIDQVYNTFDTNKDGFYDELEFIAAVNL 83

RGFLKCPTGILNVDEFKKIYANFFPYGDASKFAEHVFRTFDTNSDGTTDEREFIUALV 91

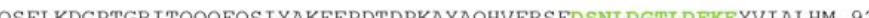

KGFLKDCPSGRLNLEEFQQLYVKFFPYGDASKFAQHAFRTFDKNGDGTIDFREFICALSI 91

KGFLKDCPSGILNLEEFQOLYIKFFPYGDASKFAQHAFRTFDKNGD GTIDFREFICALSV 91

KGFLKDCPTGHLTVDEFKKIYANFFPYGDASKFAEHVFRTFDTNGDGTIDFREFIIALSV 91

RGFKNECPSGVVNEDTFKOIYAQFFPHGDASTYAHYLFNAFDTTQTGSVKFEDFVTALSI 12 RGFKNECPSGIVNEENFKQIYSQFFPQGDSSTYATFLFNAFDTNHDGSVSFED FVAGLSV 171 RGFKNECPTGLVDEDTFKLIYAQFFPQGDATTYAHFLFNAFDADGNGAIHFEDFVVGLSI 157 RGFKNECPSGVVNEETFKEIYSQFFPQGDSTTYAHFLFNAFD TDHNGAVSFEDFIKGLSI 1 NCS1 GCAP1 GCAP2 GCAP3 Recoverin

VILIP1

VILIP2

VILIP3

KChIP1

KChIP2

KChIP4
EF-3 $\mathrm{Ca}^{2+}$ binding loop

SRGTLDEKLRWAFKLYDIDNDGYITRNEMLDIVDAIYQMVGNT--VE--LPEEENTPEK 147 VLKGKVEQKLRWYFKLYDVDGNGCIDRDELLTI IQAIRAINP--------CSDTTMTAEE 134 VLRGTLEHKLKWTFKIYDKDGNGCIDRLELLNIVEGIYQLKKACRRELQTEQGQLLTPEE 144 IMQEKMEQKLKWYFKLYDADGNGSIDKNELLDMFMAVQALNG---------QQTLSPEE 133 TSRGRLEQKLMWAFSMYDLDGNGYISREEMLEIVQAIYKMVSSV--MK--MPEDESTPEK 147 TSRGSFEQKLNWAFNMYDLDGDGKITRVEMLEIIEAIYKMVGTVTMMK--MNEDGLTPEQ 149 TTAGKTNQKLEWAFSLYDVDGNGTISKNEVLEIVMAIFKMITPEDVKL--LPDDENTPEK 150 TSRGSFEQKLNWAFEMYDLDGDGRITRLEMLEI IEAIYKMVGTVIMMR--MNQDGLTPQQ 149 TSRGKLEQKLKWAFSMYDIDGNGYISRSEMLEIVQAIYKMVSSV--MK--MPEDESTPEK 147 LLRGTVHEKLRWTFNLYDINKDGYINKEEMMDIVKAIYDMMGKYTYPV--LK--EDTPRQ 184 ILRGTVDDRLNWAFNLYDLNKDGCITKEEMLDIMKS IYDMMGKYTYPA--LR--EEAPRE 227 LLRGTVHEKLKWAFNLYDINKDGYITKEEMLAIMKS IYDMMGRHTYPI--LR--EDAPAE 213 LLRGTVQEKLNWAFNLYDINKD GYITKEEMLDIMKAIYDMMGKCTYPV--LK--EDAPRQ 207

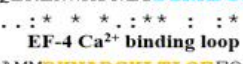

RVDRIFAMM KNAD GKLTLQEFQEGSKADPSIVQALSLYDGLV----------------- 190 FTDTVFSKIDVIGDGELSIEEFIEGVQKDQMLLDTLTRSLDLTRIVRRLQNGEQDEEGAD 194 VVDRIFLLVDENGD GQLSINEFVEGARRDKWVMKMLQMDMNPSSWLAQQRR--KSAMF-- 200 FINLVFHKID INND GEITIEEF INGMAKDQDLLEIVYKSFDFSNVLRVICNGKOPDMETD 193 RTEKIFRQMD INAND GKLSLEEFIRGAKSDPSIVRLLQCDPSSASQF---------------- 193 RAEKIWKYFGKNDDDKLTEKEFIEGT LANKEI LRLIQFEPQKVKEKM--KNA-------- 200

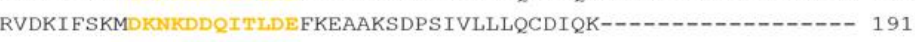
RVDKIFKKMDQDKDDQITLEEFKEAAKSDPSIVLLLQCDMQK----------------- 191 RTDKIFRQMD TNND GKL SLEEFIRGAKSDPSIVRLLQCDPSSASQF-------------- 193 HVDVFFQKMDKNKD GIVTLDEFLESCQEDDNIMRSLQLFQNVM----------------- 227 HVERFFEKMD RNQD GVVTIEEFLEACQKDENIMSSMQLFENVI------------- 256 HVESFFOKMDRNKD GVVTIEEFIESCOKDENIMRSMQLFDNVI------------------ 270 HVETFFQKMDKNKD GVVTIDEFIESCQKDENIMRSMQLFENVI------------------- 250 Hippocalcin

\section{EAAE--AAG------- 201}

SSKSPDKAGLGKVKMK 209

Figure 1.6: Sequence alignment of NCS proteins from classes A to E. Conserved residues are represented by a star, whereas asterisks indicate identical residues, colons indicate very similar residues, and periods are for weakly similar residues. 


\subsubsection{Class A: Neuronal calcium sensor 1}

Neuronal calcium sensor 1 (NCS 1 ) is the unique protein in class A. In NCS 1 protein, EF-hands 2, 3, and 4 can bind to two (Pongs et al., 1993) or three $\mathrm{Ca}^{2+}$ ions with high affinity (Ames et al., 2000; Bourne et al., 2001; Heidarsson et al., 2012). NCS 1 protein also possesses a myristoyl group located at the N-terminal domain (Figure 1.7).

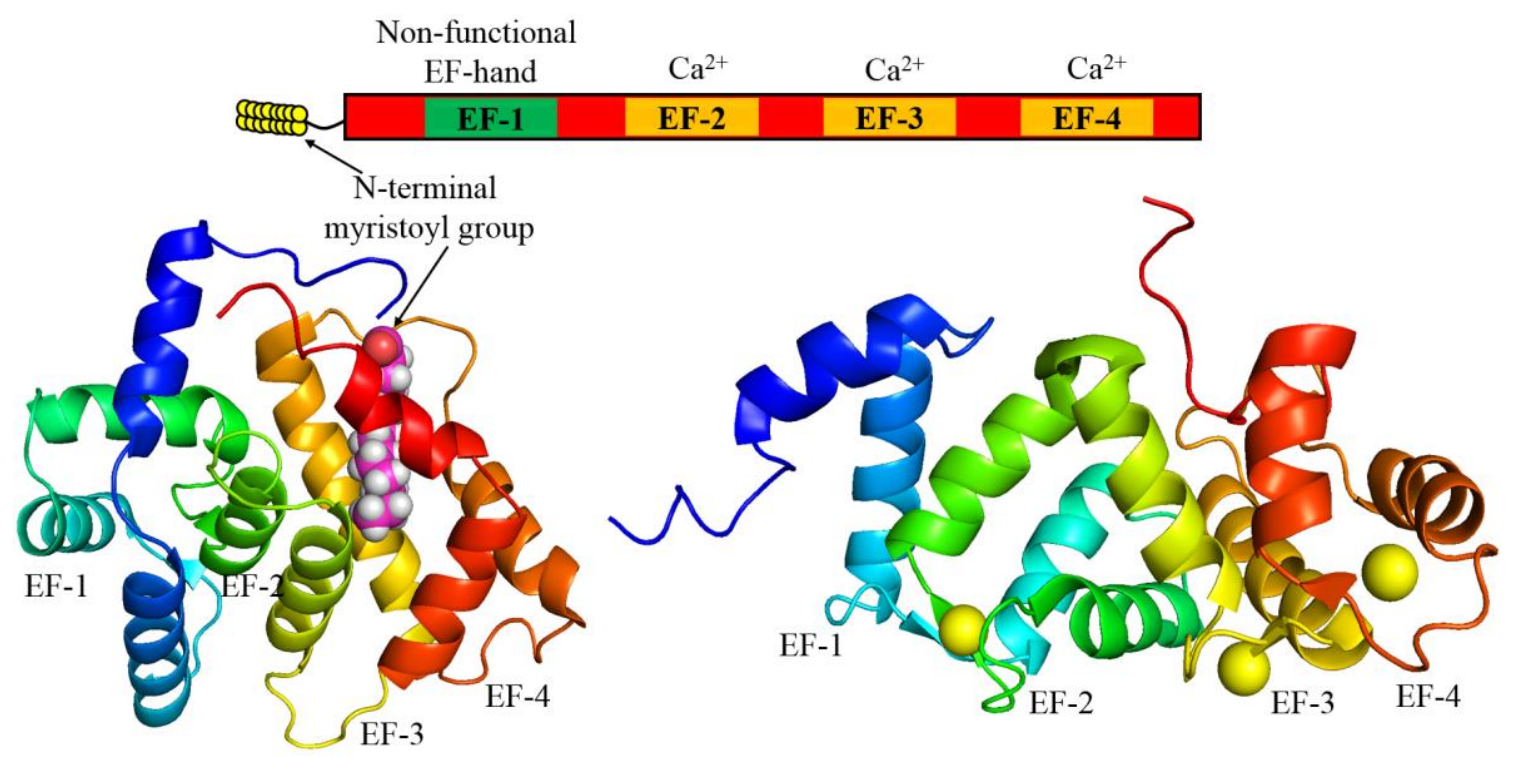

Figure 1.7: Top: Schematic diagram of EF-hands in NCS1 protein. Bottom left: structure of myristoylated ApoNCS1 (PDB code: 2L2E). Bottom right: structure of three $\mathrm{Ca}^{2+}$ bound at EF-hands 2, 3, and 4 of non-myristoylated NCS-1 (PDB code: 2LCP). The EF-hands 1, 2,3 , and 4 of NCS1 are colored in cyan, green, yellow, and red, respectively. The $\mathrm{Ca}^{2+}$ ions are the yellow spheres and the myristoyl group is colored in pink.

In most NCS proteins, extrusion of the myristoyl group allows the proteins to interact with the lipid bilayer membrane when $\mathrm{Ca}^{2+}$ is bound at EF-hands (Dason et al., 2012). In contrast, exposure of the myristoyl group in NCS 1 protein is independent of $\mathrm{Ca}^{2+}$ binding (O'Callaghan et al., 2002). However, when $\mathrm{Ca}^{2+}$ is bound, NCS 1 associates more strongly with the lipid bilayer membrane (Handley et al., 2010). The three dimensional structure of myristoylated ApoNCS 1 reveals that the myristoyl group is sequestered inside 
a cavity formed by EF-hand 3 and 4 in the C-terminal domain. In addition, these EF-hands and the fatty acyl chain are oriented in a parallel fashion, in which the myristoyl moiety is surrounded by hydrophobic residues from EF-hands (Ames et al., 2000; Ames et al., 2012). On the other hand, binding of $\mathrm{Ca}^{2+}$ at EF-hands 2, 3, and 4 in the non-myristoylated NCS 1 (Figure 1.7, left) enhances the association of NCS 1 with membranes to some degrees. Therefore, the enhancement of membrane binding observed in $\mathrm{Ca}^{2+}$ bound NCS 1 cannot be attributed solely to the $\mathrm{N}$-myristoyl moiety. However, $\mathrm{Ca}^{2+}$ induced conformational changes increase NCS 1 protein's tendency to interact with a hydrophobic surface by means independent of the N-myristoyl group.(Ames et al., 2000).

\subsubsection{Class B: visinin-like proteins, hippocalcin, and neurocalcin}

Class B of NCS proteins is comprised of three members of visinin-like proteins (VILIP) 1, 2, and 3, hippocalcin, and neurocalcin- $\delta$ protein. The proteins in class B are expressed primarily in the brain, but they are also found in peripheral organs (Braunewell et al., 2009).

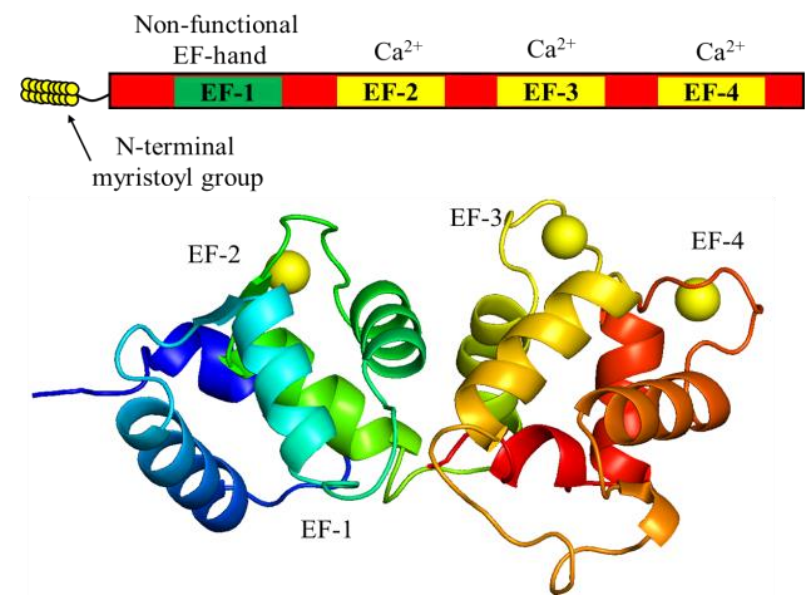

Figure 1.8: The crystal structure of $\mathrm{Ca}^{2+}$ bound non-myristoylated neurocalcin (PDB: 1BJF (Vijay-Kumar, 1999)). The EF-hands 1-4 are labeled accordingly as EF-1, EF-2, EF-3, and EF-4. The $\mathrm{Ca}^{2+}$ ion (yellow sphere) is bound at EF-2, EF-3, and EF-4. The EF-1 is disabled from binding of $\mathrm{Ca}^{2+} / \mathrm{Mg}^{2+}$ due to a Cys-Pro sequence in its $\mathrm{Ca}^{2+}$ binding loop. 
The biological functions of proteins in class B have not been well established because these proteins display differences in their $\mathrm{Ca}^{2+}$ affinities, membrane binding kinetics, and interactions with intracellular targets (Braunewell et al., 2009). It was proposed that VILIP-1 interacts with GCAPs (Spilker et al., 2002) and hippocalcin interacts with neuronal apoptosis inhibitor proteins (Lindholm et al., 2002). The 3D structure was resolved for neurocalcin and this protein exhibits similar fold as observed for other NCS proteins (Figure 1.8) (Vijay-Kumar, 1999).

\subsubsection{Class C: Recoverin}

The single protein in class $\mathrm{C}$ is recoverin. Among NCS members, recoverin was the first protein to be discovered (Dizhoor et al., 1991). It is expressed in photoreceptor cells, where it performs the main physiological function in regulation of light sensitivity by regulating rhodopsin kinase in a $\mathrm{Ca}^{2+}$ dependent manner (Calvert et al., 1995; Chen et al., 1995; Makino et al., 2004). The NMR structures of ApoRecoverin (Tanaka et al., 1995), $\mathrm{Ca}^{2+}$ bound at EF-hand 3 (Ames et al., 2002), and $\mathrm{Ca}^{2+}$ bound at both EF-hands 2 and 3 (Ames et al., 1997) were determined and are shown in Figure 1.9. Four EF-hands in recoverin are organized into $\mathrm{N}$ - and $\mathrm{C}$-terminal domains, connected together by a $\mathrm{U}$-shape linker (Ames et al., 2012). Unlike NCS1, the myristoyl group in ApoRecoverin is sequestered inside the hydrophobic cavity formed by EF-hands 1 and 2 at the $\mathrm{N}$-terminus (Tanaka et al., 1995). When $\mathrm{Ca}^{2+}$ ion is bound at EF-hand 3, the myristoyl group remains located inside the hydrophobic cavity, but the carbonyl end of the myristoyl group is displaced away from EF-hand 3 and becomes somewhat solvent exposed (Ames et al., 2012). When $\mathrm{Ca}^{2+}$ is bound at both EF-hands 2 and 3, the myristoyl group is completely forced out of the hydrophobic cavity and interacts with the lipid bilayer membrane. This 
enables recoverin to translocate from the cytosol to the disc membrane (Ames et al., 2012). Moreover, the similar structures of myristoylated and non-myristoylated $\mathrm{Ca}^{2+}$ bound recoverin suggest that the extrusion of the myristoyl group in recoverin does not depend on the interior protein structure as shown in Figure 1.9 (Ames et al., 2012).

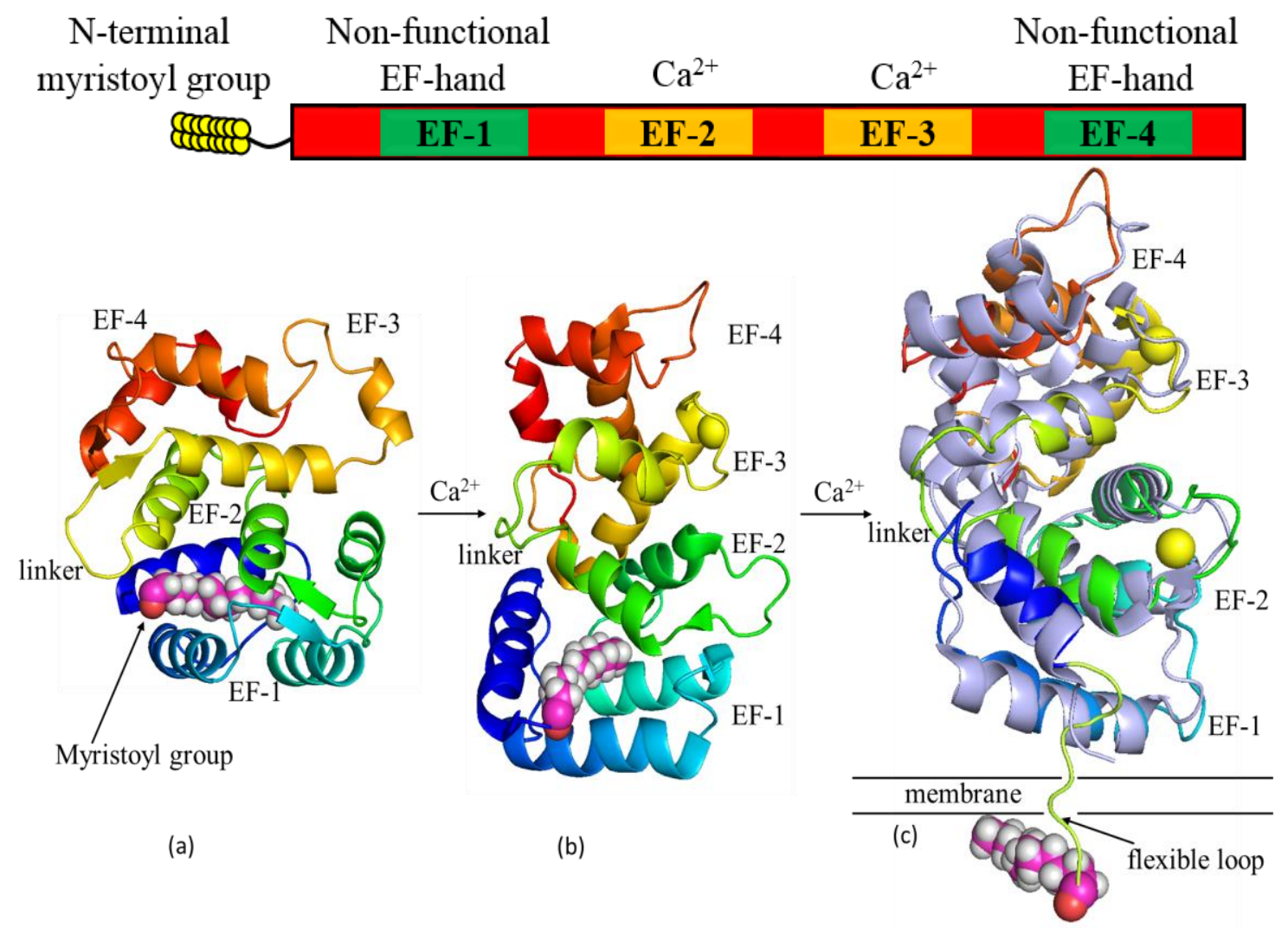

Figure: 1.9: Top: Schematic diagram of EF-hands in recoverin. Bottom: (a) structure of myristoylated ApoRecoverin (PDB code: 1IKU), (b) $1 \mathrm{Ca}^{2+}$ ion bound at EF-3 (PDB code: 1OMR), and (c) $\mathrm{Ca}^{2+}$ ion is bound at EF-hands 2 and 3 (PDB code: $1 \mathrm{JSA}$ ). The structure of two $\mathrm{Ca}^{2+}$ bound non-myristoylated recoverin is shown in light blue in (c) (PDB code: 4YI8). EF-hands 1, 2, 3, and 4 in recoverin are colored in cyan, green, yellow, and red, respectively. The $\mathrm{Ca}^{2+}$ ions (yellow spheres) are bound at EF-hand 2 and 3. The myristoyl group is shown as pink spheres and the $\mathrm{N}$-terminal eight-residue-flexible loop is highlighted in yellow. 


\subsubsection{Class D: Guanylyl cyclase activating proteins}

Proteins in class D are guanylyl cyclase activating proteins (GCAPs) which are expressed in photoreceptor cells. Their main function is to activate retinal guanylyl cyclase (RetGC) at low cytosolic $\mathrm{Ca}^{2+}$ concentration upon light activation and to inhibit the RetGC at high $\mathrm{Ca}^{2+}$ level in the dark (Dizhoor et al., 1994). Unlike recoverin, $\mathrm{Ca}^{2+}$ binding to GCAPs induces small conformational changes and thus does not force the myristoyl group out to the solvent exterior (Lim et al., 2014). In addition, EF-hands 2 and 3 in GCAPs can bind both $\mathrm{Mg}^{2+}$ and $\mathrm{Ca}^{2+}$ ions, whereas EF-hand 4 only binds to $\mathrm{Ca}^{2+}$ (Peshenko et al., 2006). Structures and molecular mechanisms of $\mathrm{Mg}^{2+}$ and/or $\mathrm{Ca}^{2+}$ binding to GCAP 1 have been well established, yet information about GCAPs 2 and 3 remains unclear. Therefore, GCAP 1 protein will be used as a model to depict the structure-function relationship of the GCAP class. Biophysical characterization of GCAP 1 shows that ApoGCAP 1 exhibits a flexible and unstructured molten-globule-like protein which does not bind and activate RetGC (Peshenko et al., 2004; Lim et al., 2009).

Binding of $\mathrm{Mg}^{2+}$ to GCAP 1 at EF-hands 2 (Figure 1.10, bottom left) stabilizes the protein tertiary structure which allows the protein to interact with and activate RetGC. Binding of $\mathrm{Ca}^{2+}$ to GCAP 1 at EF-hands 2, 3, and 4 (Figure 1.10 bottom right) induces a distinct conformation that inhibits RetGC (Dizhoor et al., 1994; Peshenko et al., 2004; Peshenko et al., 2006; Lim et al., 2009; Lim et al., 2014). 


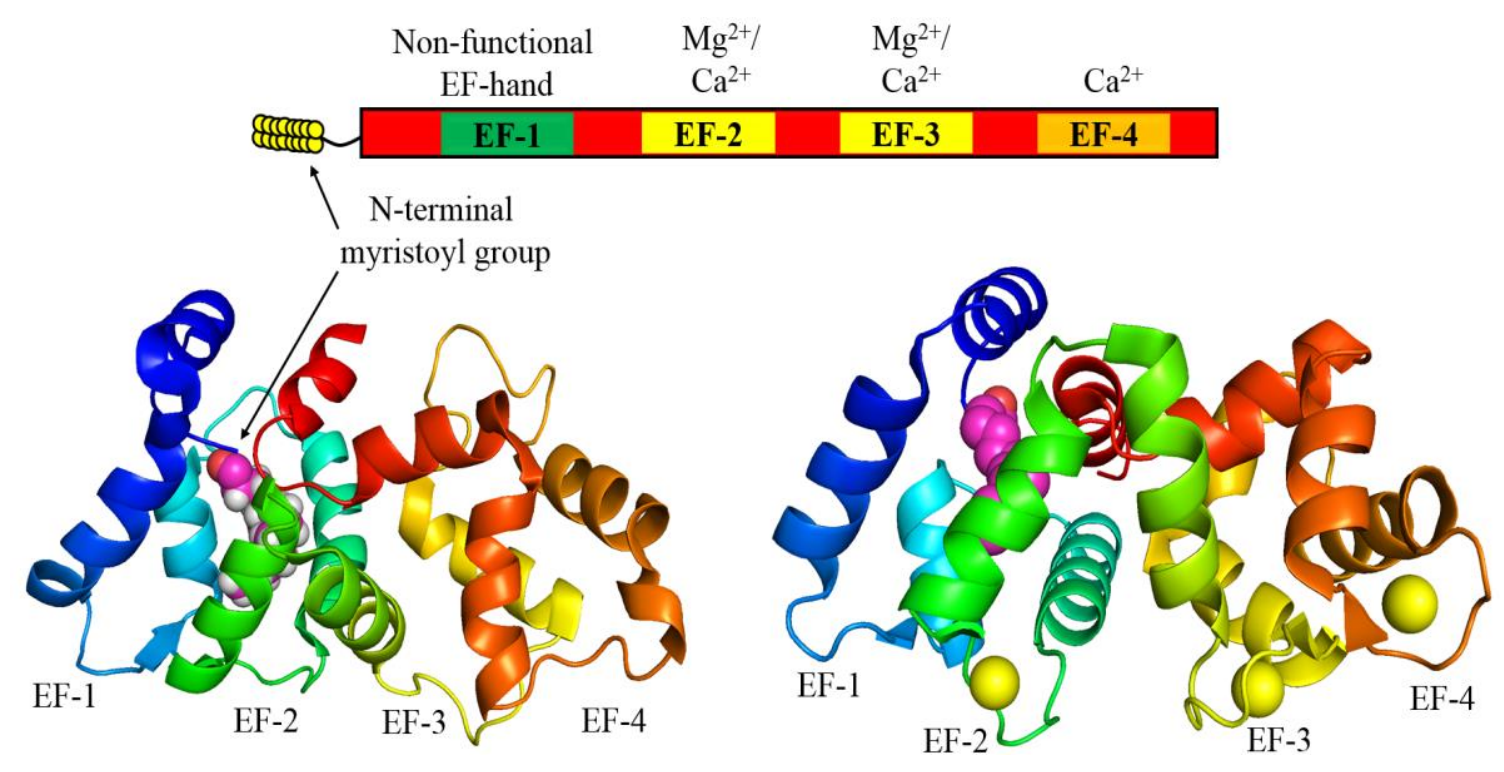

Figure 1.10: Top: Schematic diagram of EF-hands in GCAPs. Bottom: (left) structure of $\mathrm{Mg}^{2+}$ bound at EF-hand 2 of myristoylated GCAP 1 mutant V77E $\left(\mathrm{Mg}^{2+}\right.$ is not shown, PDB code: 2NA0) and (right) structure of $\mathrm{Ca}^{2+}$ bound at EF-hands 2, 3, and 4 in GCAP 1 (PDB code: 2R2I). EF-hands 1 to 4 are highlighted in cyan, green, yellow, and red, respectively. The $\mathrm{Ca}^{2+}$ ions are shown as yellow spheres.

\subsubsection{Class E: Potassium channel interacting proteins}

The proteins in class $\mathrm{E}$ are comprised of potassium channel interacting proteins (KChIP) 1-4. The KChIPs share a high sequence similarity over the four EF-hands with about $40 \%$ identity, whereas the N-terminal sequence is considerably diverse (Burgoyne, 2004). Alternative splicing of the four human KChIP genes generates different protein variants with diverse $\mathrm{N}$-terminal domains. Some of the isoforms have myristoylation or palmitoylation sites that assist membrane association and localization (Figure 1.11). However, the topology for KChIP isoforms remains unknown (Burgoyne, 2004). The four KChIP proteins and additional splice variants are expressed in neuronal cells and KChIP2 is also expressed in cardiac myocytes (An et al., 2000; Morohashi et al., 2002; Holmqvist et al., 2002). The expression patterns of KChIPs have considerable importance for 
understanding diverse functions of KChIP proteins in modulation of different aspects of neuronal regulation.

\begin{tabular}{|c|c|c|c|c|c|}
\hline cescen & EF-1 & EF-2 & EF-3 & EF-4 & KChIP1.1 \\
\hline & EF-1 & EF-2 & EF-3 & EF-4 & KChIP1.2 \\
\hline & EF-1 & EF-2 & EF-3 & EF-4 & KChIP1.3 \\
\hline & EF-1 & EF-2 & EF-3 & EF-4 & KChIP2.1 \\
\hline & EF-1 & EF-2 & EF-3 & EF-4 & $\mathrm{KChIP2.2}$ \\
\hline & EF-1 & EF-2 & EF-3 & EF-4 & $\mathrm{KChIP2.3}$ \\
\hline & EF-1 & EF-2 & EF-3 & EF-4 & KChIP2.4 \\
\hline & EF-1 & EF-2 & EF-3 & EF-4 & KChIP2.5 \\
\hline & EF-1 & EF-2 & EF-3 & EF-4 & KChIP3.1 \\
\hline & EF-1 & EF-2 & EF-3 & EF-4 & KChIP3.2 \\
\hline & EF-1 & EF-2 & EF-3 & EF-4 & KChIP4.1 \\
\hline & EF-1 & EF-2 & EF-3 & EF-4 & KChIP4.2 \\
\hline & EF-1 & EF-2 & EF-3 & EF-4 & KChIP4.3 \\
\hline & EF-1 & EF-2 & EF-3 & EF-4 & KChIP4.4 \\
\hline & EF-1 & EF-2 & EF-3 & EF-4 & KChIP4.5 \\
\hline & EF-1 & EF-2 & EF-3 & EF-4 & KChIP4.6 \\
\hline $\begin{array}{l}\text { N-ter } \\
\text { varia }\end{array}$ & & $\mathrm{Col}$ & do & 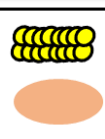 & stoylation sit \\
\hline
\end{tabular}

Figure 1.11: KChIPs and splice variants in class E. Some KChIP isoforms have myristoylation or palmitoylation sites, assisting membrane association and localization. The GenBank accession number for human KChIP1 isoforms 1.1, 1.2, 1.3 DQ148478, DQ148477, and DQ148476. The GenBank accession number for human KChIP2 isoforms 2.1, 2.2, 2.3, 2.4, and 2.5 are NM-01491, DQ148480, DQ148481, DQ148482, and DQ148483. The GenBank accession number for human KChIP3 isoforms 3.1 and 3.2 are DQ148485 and DQ148486. The GenBank accession number for human KChIP4 isoforms are DQ148487, DQ148488, DQ148491, DQ148489, DQ148490, and DQ148492, respectively. Adapted from (Burgoyne, 2007).

The crystallographic study revealed that KChIP1 interacts with the N-terminal of $\mathrm{Kv} 4.3$ results in an octomeric structure of four primary Kv4 subunits and four KChIP subunits forming heteromultimeric $\mathrm{Kv}$ channels. The crystal structure reveals that a 
monomer of KChIP1 interacts with two T1 domains of Kv4.3 (Pioletti et al., 2006). The first binding site is through hydrophobic interaction between the N-terminus of Kv4.3 and the hydrophobic cavity on the KChIP1 surface, being exposed upon $\mathrm{Ca}^{2+}$ association to KChIP1 (Pioletti et al., 2006). The second binding site is through the helix 2 in KChIP1 and the residues in the loop of $\mathrm{T} 1$ domain (Pioletti et al., 2006). The interaction between KChIP1 and Kv4 channel is $\mathrm{Ca}^{2+}$ independent, however, binding of $\mathrm{Ca}^{2+}$ to KChIP1 promotes the channel activity (An et al., 2000; Pioletti et al., 2006).

\subsubsection{KChIP3/DREAM/calsenilin}

Among proteins in class E, KChIP3 is also known as calsenilin or downstream regulatory element antagonist modulator (DREAM) protein according to their identical gene sequence (Buxbaum et al., 1998; Carrion et al., 1999; An et al., 2000). Because of that, herein DREAM is used to avoid using different names for the same protein.

The DREAM protein has been shown to be a multifunctional neuronal calcium sensor protein that is expressed in the central nervous system, thyroid glands, immune system and testis (Carrion et al., 1999). The physiological functions of DREAM are involved in various interactions with the targeted DNA and proteins both in and outside of the nucleus. In the cytoplasm, KChIP3 interacts with $\mathrm{Kv} 4.3$ potassium channels to modulate A-type potassium currents (An et al., 2000). KChIP3 also interacts with the Cterminal fragments of presenilins to facilitate the activity of gamma-secretase and increase the production of $\beta$-amyloid plaques in Alzheimer's disease (Buxbaum et al., 1998). In the

nucleus, $\mathrm{Ca}^{2+}$ free KChIP3 directly binds to specific downstream regulatory elements of the prodynorphin and c-fos genes to repress the transcription of these genes. The DREAM protein also interacts with a silencer sequence in the 3' untranslated region of the apoptotic 
gene $h r k$ and represses the expression of the downstream reporter genes in $\mathrm{Ca}^{2+}$ dependent manner (Sanz et al., 2001). In addition, at low level intracellular $\mathrm{Ca}^{2+}$ concentration, the two leucine-charged residue-rich domains (LCDs) in DREAM interact with the two LCDs located in the kinase-inducible domain and in the leucine zipper domain of aCREM, resulting in the loss of DREAM binding to DRE sites and derepression (Ledo et al., 2002).

\subsubsection{Structural insight from DREAM}

The DREAM protein contains 256 amino acid residues ( $29 \mathrm{kDa}$ ) (Carrion et al., 1999; Craig, et al., 2002), which constitute four EF-hands and a non-conserved N-terminal region (residues 1 - 64) as shown in Figure 1.12 (Buxbaum et al., 1998; Carrion et al., 1999; An et al., 2000). The N-terminal extension region contains a greater number of hydrophobic residues that cause inclusion body formation and aggregation during protein folding (Fink, 1998, Osawa et al., 2001). However, deletion of this region (residues 1-64) does not inhibit binding of DNA or dimerization in DREAM (Osawa et al., 2001; Osawa et al., 2005; Lusin et al., 2008). Furthermore, the truncated construct of DREAM (residues 65-265) also fully activates the Kv4 channels (An et al., 2000).

Structural characterization of DREAM reveals that EF-hand 1 does not bind $\mathrm{Ca}^{2+}$ or $\mathrm{Mg}^{2+}$ because of the conserved sequence $\mathrm{CPxG}$ (Figure 1.12) in the first $\mathrm{Ca}^{2+}$ binding loop similar to that obtained in the crystal structure of recoverin (Flaherty et al., 1993), KChIP1 (Zhou et al., 2004; Scannevin et al., 2004), frequenin (Bourne et al., 2001), and neurocalcin (Vijay-Kumar et al., 1999). Substitution of the glutamic acid residue by an aspartic acid residue at the twelfth position in the $\mathrm{Ca}^{2+}$ binding loop of EF-hand 2 causes this site to selectively bind to $\mathrm{Mg}^{2+}$ with a high affinity $\left(\mathrm{K}_{\mathrm{d}} \sim 13 \mu \mathrm{M}\right)$ (da Silva et al., 1995, Craig et al., 2002, Osawa et al., 2005). On the other hand, EF-hands 3 and 4 exclusively 
bind to $\mathrm{Ca}^{2+}$ with a relatively high affinity $\left(\mathrm{K}_{\mathrm{d}} \sim 1 \mu \mathrm{M}\right)$ due to the conserved glutamic acid residue at the twelfth position in the $\mathrm{Ca}^{2+}$ binding loop of EF-hands 3 and 4 (Figure 1.12)

(Osawa et al., 2001).

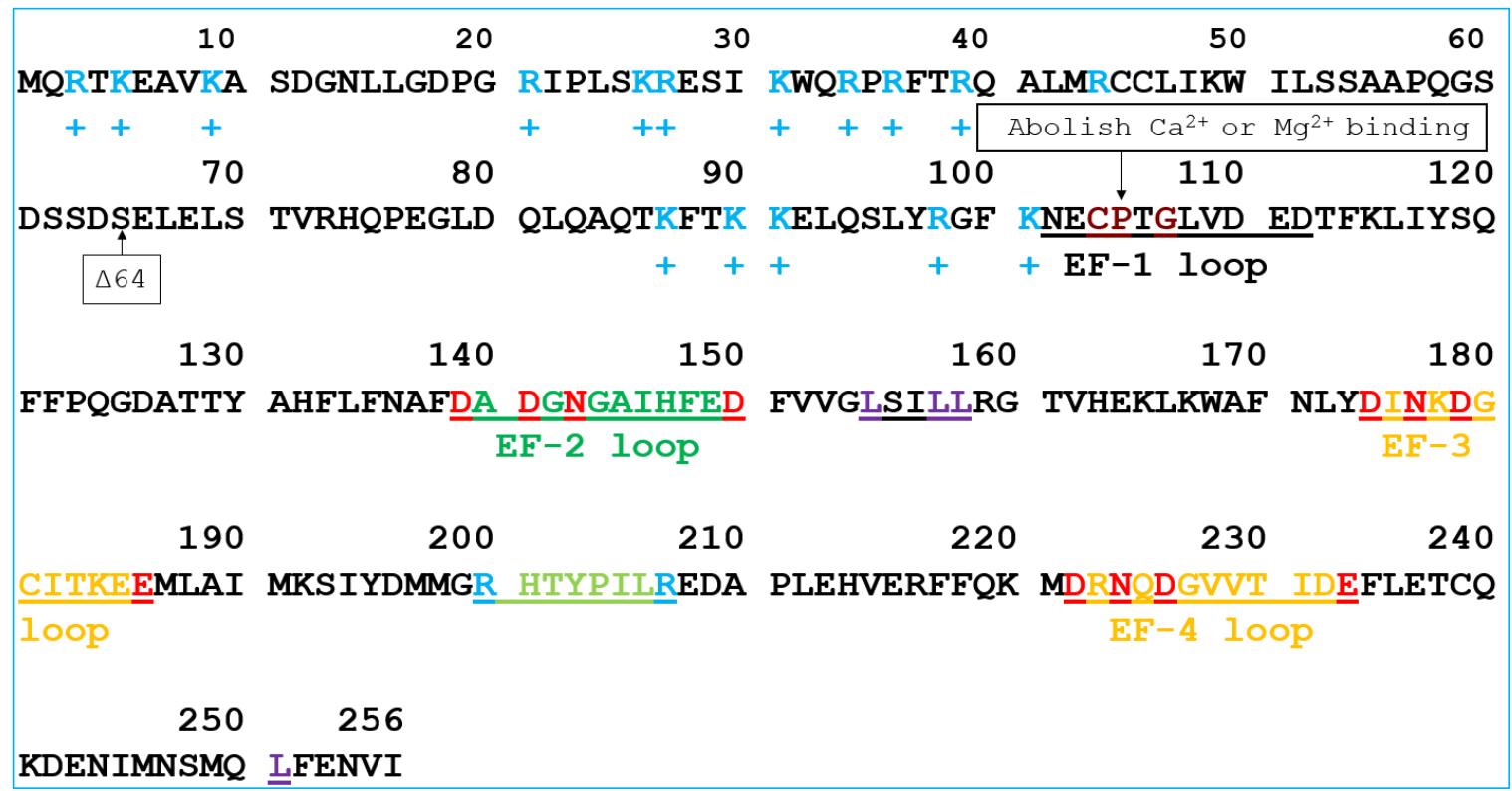

Figure 1.12: The amino acid sequence of mouse DREAM (Gene code: Q9QXT8). The $\mathrm{Ca}^{2+}$ and/or $\mathrm{Mg}^{2+}$ binding loops of EF-hands 1, 2, 3, and 4 are underlined. The negatively charged amino acids involved in $\mathrm{Ca}^{2+} / \mathrm{Mg}^{2+}$ coordination are shown in red. The conserved sequence $\mathrm{CPxG}$ (in brown) in the first EF-hand eliminates binding of $\mathrm{Ca}^{2+} / \mathrm{Mg}^{2+}$ at this site. The flexible loop connecting $\alpha 7$ from EF-hands 3 and $\alpha 8$ from EF-hand 4 is shown in light green and underlined, while the positively residues R200 and R207 in the loop are shown in blue. The leucine residues (155LxxLL159, and L251) at $\mathrm{Ca}^{2+}$ DREAM dimeric interface are highlighted in purple.

The three-dimensional NMR structure of $\mathrm{Ca}^{2+}$ bound DREAM (residues 76 - 256, PDB entry: 2JUL (Lusin et al., 2008)) as shown in Figure 1.13 reveals that DREAM consists of $10 \alpha$-helices, evenly distributed into the $\mathrm{N}$ - and C-terminal domains. The Nterminal domain comprises EF-hand 1 ( $\alpha 2$ and $\alpha 3$, residues 90 - 120) and EF-hand $2(\alpha 4$ and $\alpha 5$, residues 128 - 159). The C-terminal domain consists of EF-hand 3 ( $\alpha 6$ and $\alpha 7$, residues 163 - 198) and EF-hand 4 ( $\alpha 8$ and $\alpha 9$, residues 184 - 240) (Figure 1.13) (Lusin et al., 2008). 


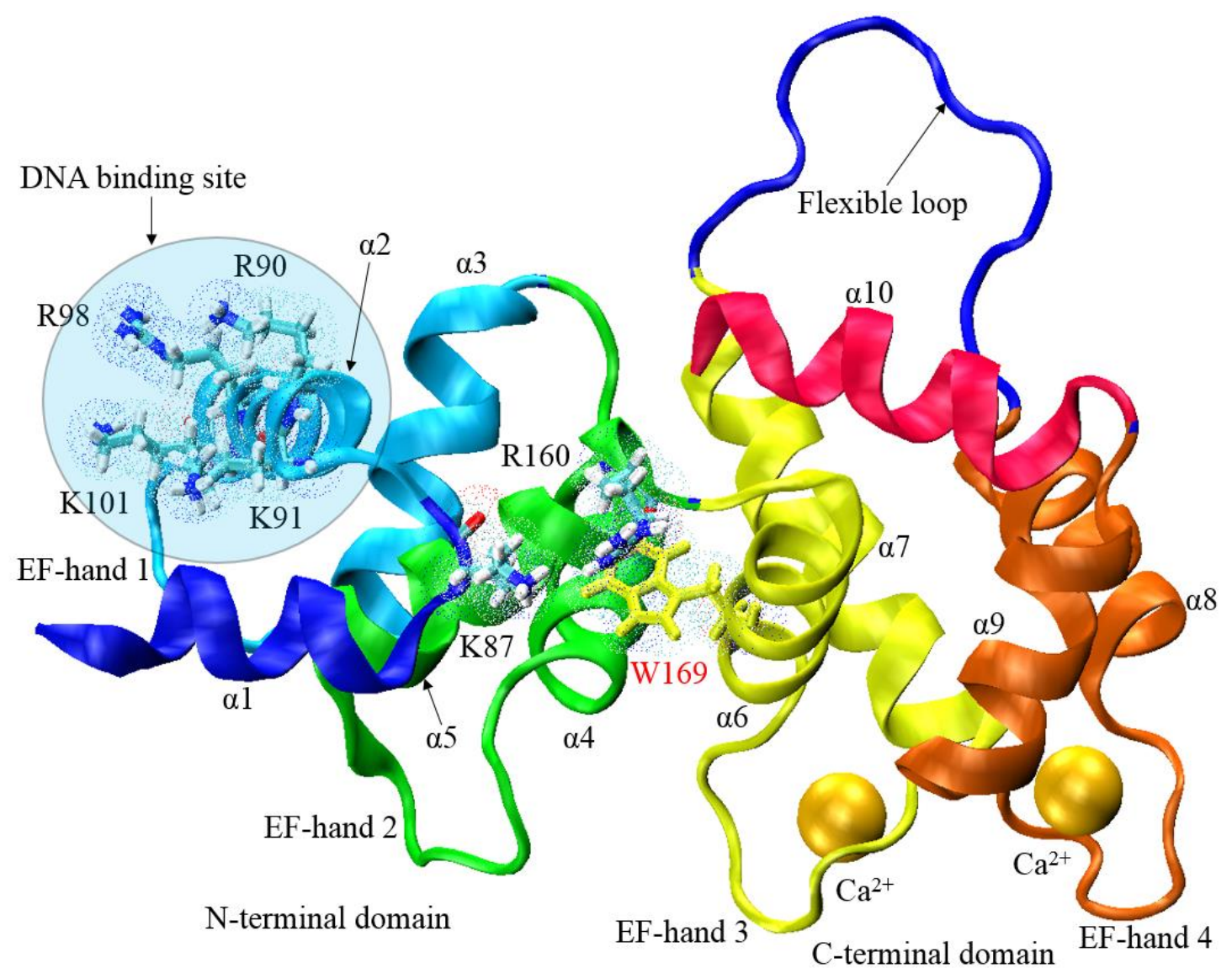

Figure 1.13: Three dimensional cartoon structure of $\mathrm{Ca}^{2+} \mathrm{DREAM}$ (PBS: 2JUL, residues 76 - 256) (Lusin et al., 2008). The structure DREAM consists of $10 \alpha$-helices (43\% secondary structure content) connected by random coils, turns, and a few beta sheets (2\%). From left to right, the positively charged residues postulated to facilitate DNA binding (K87, K90, K91, R98, K101, R160) are shown in licorice. The EF-hands 1, 2, 3, and 4 are shown in cyan, green, yellow, and orange, respectively. The N-terminal $\alpha$-helix $(\alpha 1)$ and the C-terminal alpha helix $(\alpha 10)$ are labeled in blue and red, respectively. The single intrinsic tryptophan residue (W169) is shown in yellow licorice. The long flexible loop connecting $\alpha 7$ and $\alpha 8$ is shown in blue. The $\mathrm{Ca}^{2+}$ ion (yellow sphere) is bound to EF-hands 3 and 4.

The two domains in $\mathrm{Ca}^{2+}$ DREAM are linked together through the central hydrophobic cluster comprising of residues Y130, F133, and A137 from $\alpha 4$ and residues W160, L173, I190, and M197 from $\alpha 6$ and $\alpha 7$ of DREAM (Figure 1.14). 


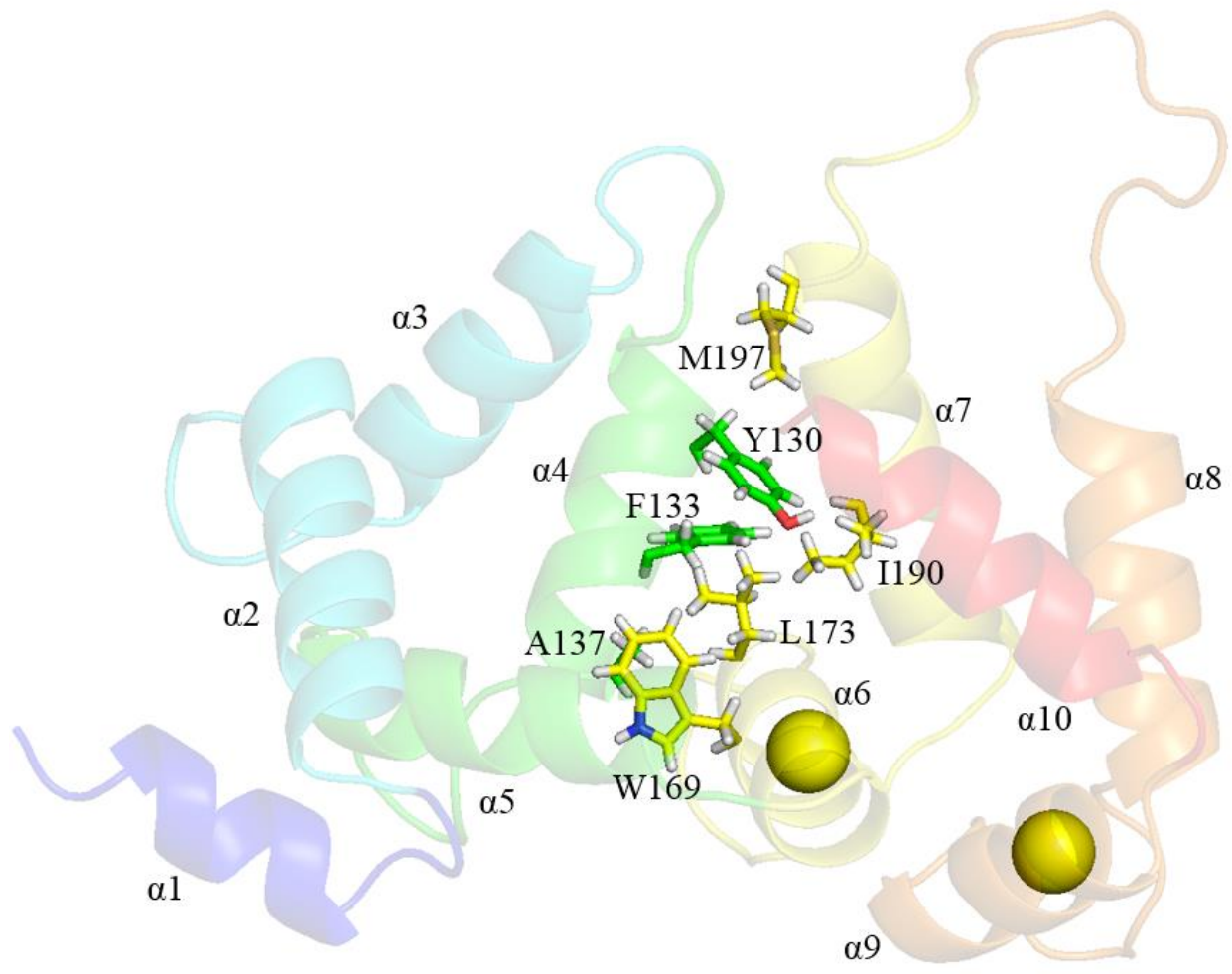

Figure 1.14: The central hydrophobic cluster (shown in stick) connecting the $\mathrm{N}$ - and the Cterminal domains of DREAM (PDB: 2JUL) (Lusin et al., 2008).

Moreover, another hydrophobic cluster was also determined at the C-terminal domain of $\mathrm{Ca}^{2+}$ DREAM, comprising of residues M246, M249, F252, and I256 from $\alpha 10$ (in red), residues L167, A170, M191, and L194 from EF-hand 3 (in yellow) and residues F218, and M222 from EF-hand 4 (in orange) of DREAM (Figure 1.15). The hydrophobic clusters in DREAM structure were shown to be essential for DREAM interactions with the $\mathrm{T} 1$ domain of $\mathrm{K}_{\mathrm{V}} 4.3$ channels as well as with small hydrophobic molecules as arachidonic acid and NS5806. (Gonzalez and Miksovska, 2014; Gonzalez, Pham et al., 2014) 


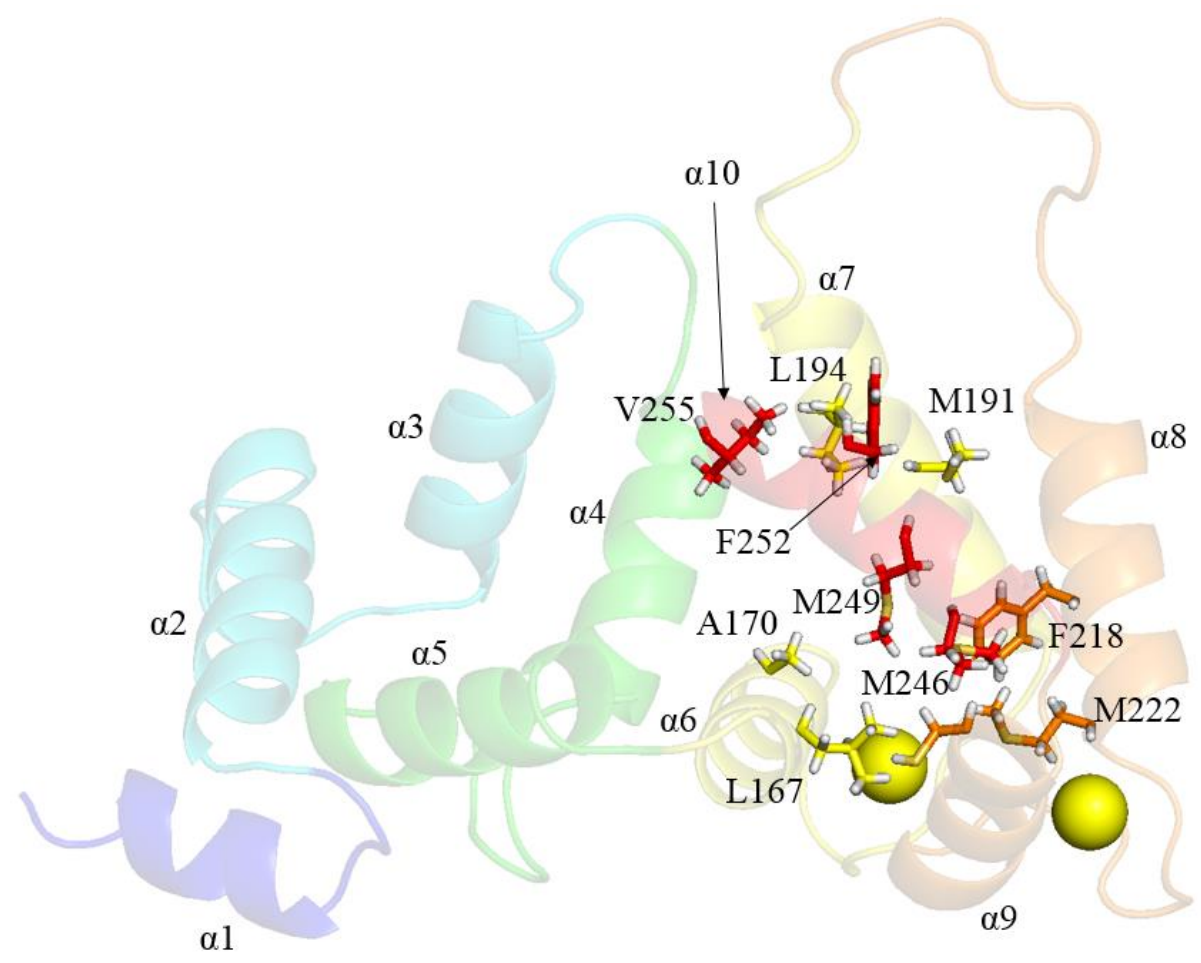

Figure 1.15: The C-terminal hydrophobic cluster (as shown in stick) of DREAM (Lusin et al., 2008).

\subsubsection{The $\mathrm{Ca}^{2+}$ and $\mathrm{Mg}^{2+}$ binding properties in DREAM}

The binding affinity for $\mathrm{Mg}^{2+}$ and $\mathrm{Ca}^{2+}$ in DREAM has been studied intensively. Craig et al. demonstrated that one $\mathrm{Ca}^{2+}$ binds to the first EF-hand in DREAM with a high affinity $\left(\mathrm{K}_{\mathrm{d}} \sim 0.6 \mu \mathrm{M}\right)$ and another $\mathrm{Ca}^{2+}$ binds to the second EF-hand with a lower affinity, with a $\mathrm{K}_{\mathrm{d}}$ ranging from 1 to $10 \mu \mathrm{M}$, relative to that of the first EF-hand (Craig et al., 2002). Osawa et al. showed that the first $\mathrm{Ca}^{2+}$ ion binds to EF-hand 3 and the second $\mathrm{Ca}^{2+}$ ion subsequently binds to EF- hand 4 in DREAM (Osawa et al., 2005). On the other hand, EFhand 2 in DREAM selectively binds to $\mathrm{Mg}^{2+}$ with a high affinity $\left(\mathrm{K}_{\mathrm{d}} \sim 13 \mu \mathrm{M}\right)$ (Osawa et al., 2005; Lusin et al., 2008). Binding of $\mathrm{Ca}^{2+}$ has been shown to induce the dimeric formation of DREAM. However, our current knowledge of the mechanism of $\mathrm{Ca}^{2+}$ induced 
dimeric formation is limited. The proposed structure of the DREAM dimer was determined according to the directed mutational studies by Lusin et al. 2008 as well as the crystal structure of KChIP1 (Zhou et al., 2004). By using size-exclusion chromatography and pulsed-field gradient diffusion studies, Osawa et al. 2005 showed that the association of $\mathrm{Ca}^{2+}$ stabilizes a dimeric DREAM. Lusin et al. 2008 derived a model for dimeric DREAM in the $\mathrm{Ca}^{2+}$ bound form using the dimeric X-ray structure of KChIP1 (Zhou et al., 2004). In this model, the residues L155, L158, and L159 (155LxxLL159, x: any amino acid residue) on the N-terminal domain of the first DREAM molecule interact with residue L251 on the C-terminal domain of the second DREAM molecule and these two DREAM monomers are oriented in the head-to-tail orientation (Lusin et al., 2008). Lusin et al. 2008 also speculated that residues in the C-terminal domain of the first DREAM molecule cover the positively charged residues K90, K91, R98, and K101 in $\alpha 2$ of the second DREAM molecule, subsequently eliminating the interaction of DREAM with DNA. In general, binding of $\mathrm{Ca}^{2+}$ to DREAM induces significant conformational changes. However, the proposed model structure of DREAM dimer has never been verified in experimental studies. In addition, significantly less is known about the impact of $\mathrm{Mg}^{2+}$ and/or $\mathrm{Ca}^{2+}$ on DREAM conformational dynamics as well as the role of conformational dynamics in DREAM upon interaction with target proteins or DNA. 


\section{OBJECTIVES}

Understanding the conformational dynamics and stability associated with $\mathrm{Ca}^{2+}$ and/or $\mathrm{Mg}^{2+}$ binding to DREAM is crucial for delineating the structure-dynamics-function relationship of DREAM, as well as for elucidating the mechanisms of interaction of DREAM with DNA or presenilins. The critical barrier for envisioning the molecular mechanism of the interaction between DREAM and its interacting partners lies in the lack of NMR/crystal structures of the Apo and $\mathrm{Mg}^{2+} \mathrm{DREAM}$. The primary goal of this project was to delineate the structure-dynamics-function relationship of DREAM upon binding with $\mathrm{Ca}^{2+}$ and/or $\mathrm{Mg}^{2+}$ in modulation of the interaction with presenilin 1 and DNA. To accomplish this goal, I employed a combination of fluorescence spectroscopy, circular dichroism, isothermal titration calorimetry, molecular dynamics simulation, and computational docking to investigate the impact of $\mathrm{Mg}^{2+}$ and/or $\mathrm{Ca}^{2+}$ on the conformational dynamics of DREAM, the functional roles of individual EF-hands in DREAM, and the binding interface between DREAM and DNA as well as between DREAM and presenilin 1.

Here, I hypothesize that the structural changes associated with $\mathrm{Ca}^{2+}$ binding to $\mathrm{EF}$ hands 3 and 4 are propagated from the $\mathrm{C}$-terminal domain into the $\mathrm{N}$-terminal domain leading to the dissociation of the DREAM-DNA complex. In addition, I propose that there is a high affinity binding site for DNA that is formed by the interfacing of residues from DREAM with nucleotides at the target DRE site in prodynorphin gene for DREAM. Finally, I speculate that residues in the hydrophobic cavity at the C-terminal domain and residues in the long flexible loop connecting $\alpha 7$ and $\alpha 8$ in DREAM interface with residues from the C-terminal fragment of presenilin 1. Findings from this project will provide 
insight into the molecular interaction of DREAM with presenilins and DNA at the amino acid residue level. These findings also provide insight into the roles of individual EF-hands in DREAM in regulating DREAM conformational transition under physiological conditions, which can be used as a model to uncover the functional properties of EF-hands in other neuronal calcium sensor proteins and to characterize the effects of $\mathrm{Mg}^{2+}$ on various $\mathrm{Ca}^{2+}$ signaling pathways in cells. These results are also important to understand the role of DREAM under physiological and pathological conditions and may provide a structure based design of a novel drug compounds to treat Alzheimer's disease and chronic pain. To accomplish the research goals, the following objectives were carried out.

\subsection{Objective 1}

Characterize the structural changes associated with $\mathrm{Mg}^{2+} / \mathrm{Ca}^{2+}$ binding to DREAM wild-type (residues 65 - 256), DREAM C-terminal domain (namely DREAM-C, residues 161 - 256), and DREAM EF-hand mutants (inactive EF-hand 2: D150 $\rightarrow \mathrm{N}$, inactive EFhand 3: E186 $\rightarrow$ Q, and inactive EF-hand 4: E234 $\rightarrow$ Q)

Previous studies have shown that $\mathrm{Ca}^{2+}$ binds to ApoDREAM tetramer promoting DREAM dimerization, whereas the $\mathrm{Mg}^{2+}$ binds to DREAM leading to an equilibrium between tetramer and monomer of the protein. However, the detailed mechanism of $\mathrm{Ca}^{2+} / \mathrm{Mg}^{2+}$ induced structural/dynamic changes remains unknown. To characterize the conformational dynamics associated with $\mathrm{Ca}^{2+} / \mathrm{Mg}^{2+}$ binding to DREAM protein, I took advantage of the single intrinsic tryptophan residue (W169) located in the central hydrophobic pocket between EF-hand 2 and 3 to monitor conformational changes in DREAM, DREAM-C, and DREAM EF-hand mutants (namely DREAM-D150N, DREAM-E186Q, and DREAM-E234Q for DREAM mutants with an inactive EF-hand 2, 
3 , or 4, respectively) using fluorescence steady-state and time-resolved spectroscopy. In addition, DREAM stability in the Apo and $\mathrm{Ca}^{2+}$ and/or $\mathrm{Mg}^{2+}$ bound forms were probed in unfolding studies. The outcomes of this part in the project are described in chapter 4 (published in Pham et al., 2015), chapter 5, and partially in chapter 7.

\subsection{Objective 2}

Characterize the molecular mechanism between DREAM and presenilin 1 as well as the binding interface of the DREAM-presenilin 1 C-terminal fragment complex.

Previous studies demonstrated that DREAM interacts with the C-terminal fragment of presenilins 1 and 2 leading to the formation of $\mathrm{A} \beta$ plaques in Alzheimer's disease. However, insight into the molecular mechanism of the interaction between DREAM and presenilins as well as the impact of $\mathrm{Mg}^{2+}$ and/or $\mathrm{Ca}^{2+}$ on the complex formation remain unknown. The binding between DREAM and presenilin 1 C-terminal fragment (PS1-CTF) was probed by determining the binding constant between DREAM and peptides constituting the PS1-CTF using steady-state and time-resolved fluorescence spectroscopy. Subsequently, the oligomerization of the complex was characterized using time-resolved polarization. The residues at DREAM-presenilin binding interface were identified using computational approaches. The results of this part in the project are described in chapter 6 (published in Pham and Miksovska, 2016).

\subsection{Objective 3}

Determine the functional roles of individual EF-hands in DREAM as well as elucidate molecular mechanism of interaction between DREAM and DNA.

Previous studies revealed that ApoDREAM tetramer binds to the DRE sequence of prodynorphin gene (namely DNA), whereas association of $\mathrm{Ca}^{2+}$ at either EF-hand 3 or 4 in 
DREAM promotes DREAM dimerization and dissociates the DREAM-DNA complex. However, in the absence of ApoDREAM structure, the mechanism of interaction between DREAM and DNA remains unclear. To accomplish this goal, I employed isothermal titration calorimetry (ITC) to determine thermodynamic parameters for DREAM and DREAM mutants binding to 25-oligomer DNA. Furthermore, the interaction of DREAM and DNA was also probed using time-resolved anisotropy to monitor oligomeric states of the complex upon $\mathrm{Mg}^{2+}$ and $\mathrm{Ca}^{2+}$ association. Previously, according to the structure of DREAM monomer, it was proposed that basic and positively charged residues K90, K91, Q94, R98, and K101 in $\alpha 2$ of DREAM form hydrogen bonds and electrostatic interactions with DNA (Lusin et al., 2008). The binding interface of the ApoDREAM-DNA complex as well as the conformational dynamics of ApoDREAM upon DNA association were also examined using computational approaches. The results of this part in the project are described in chapter 7.

\subsection{Objective 4}

Characterize the kinetics and thermodynamics associated with $\mathrm{CO}$ binding or releasing from horse heart myoglobin (Mb) and microperoxidase-11 (MP-11) encapsulated in sodium bis-(2-ethylhexyl) sulfosuccinate (AOT) reverse micelles: A model system to study myristoylated and palmitoylated NCS proteins in their lipid membrane association state.

DREAM and most NCS proteins possess either a myristoylation or a palmitoylation site at their N-terminus (Burgoyne, 2007), which serves as an element for membrane targeting and anchoring of proteins (Valentine et al., 2010). Understanding membrane

association, controlled by $\mathrm{Ca}^{2+}$ triggered exposure of the myristoylation/palmitoylation, is 
crucial to delineate protein-protein interactions mediating signal transduction at the membrane (Valentine et al., 2010). However, characterizations of the conformational dynamics of DREAM and other NCS proteins at $\mathrm{Ca}^{2+}$ saturated state are often frustrated due to the poor solubility of these proteins (Tanaka et al., 1995; Ames et al., 1997; Valentine et al., 2010). One of the common approaches to obtain a highly soluble and wellbehaved protein is to delete the myristoylation/palmitoylation region containing hydrophobic residues as done in DREAM (the most common form of purified DREAM protein is the construct containing residues $65-256)$. However, this approach prevents our understanding of the role of the myristoylation/palmitoylation site in the DREAM interactions with targeted proteins. The long term goal of this project is to investigate the conformational dynamics of DREAM full length construct (residues 1 - 256) in its membrane-anchored state and the interaction of presenilins with DREAM full length encapsulated in reverse micelles. The most critical and difficult stage of this project is to develop a reverse micelle encapsulation model to investigate the impacts of aqueous solvation and confinement on the structure, stability, and dynamics of full length DREAM. Therefore, at the beginning stage of this project, I investigated the conformational dynamics of myoglobin $(\mathrm{Mb})$ and microperoxidase-11 (MP-11) proteins in the restricted environment of reverse micelles. Photoacoustic calorimetry (PAC) and transient absorption (TA) were used to characterize the kinetics and thermodynamics associated with carbon monoxide (CO) binding or releasing from the heme pocket of horse heart Mb and MP-11. The $\mathrm{Mb}$ and MP-11 proteins were chosen as model proteins for this study because the mechanism of $\mathrm{CO}$ binding or releasing from the heme group of these proteins is well understood. In addition, these proteins are highly soluble and stable in buffer solution. 
Therefore, the characterization of conformational dynamics of $\mathrm{Mb}$ and MP-11 encapsulated in reverse micelles is a good model system to study $\mathrm{Ca}^{2+}$ triggered exposure of palmitoylation in DREAM encapsulated in reverse micelles upon photolysis of caged $\mathrm{Ca}^{2+}$ compound. The results of this part are described in chapter 8 (partially published in reference (Larsen et al., 2011)). 


\section{MATERIALS AND METHODS}

\subsection{Materials}

The plasmids encoding for constructs of mouse DREAM wild-type (DREAM, residues 65 - 256), DREAM-C terminal domain (DREAM-C, residues 161 - 256), DREAM-EF hand mutants: DREAM-D150N, DREAM-E186Q, and DREAM-E234Q (where the EF-hands 2, 3, and 4 in DREAM are inactivated by site-direct mutations D150 $\rightarrow \mathrm{N}, \mathrm{E} 186 \rightarrow \mathrm{Q}$, and E234 $\rightarrow$ Q, respectively), DREAM-KChIP1, and DREAM-NCS1 chimera (where DREAM construct fused with KChIP1 or NCS1 flexible loop) with T7 promoter and six His tagged on the carboxyl terminus were purchased from GeneCopoeia (Rockville, MD, USA). The DNA sequence '5-

CAGGGGCCTCCTTGACTCCGGCTTC-3' and its complement sequence corresponding to downstream regulatory elements (DRE) of human prodynorphin gene (namely DNA) were also purchased from GeneCopoeia. The synthetic peptides derived for HL $\beta$ (residues 355PESRAAVQELSSSIL369) and HL9 (residues 445FYFATDYLVQPFMDQLAFHQFYI467) of human presenilin 1 C-terminal fragment (herein named HL $\beta$ and HL9) with FITC covalently attached to their amino-terminus were purchased from ProImmune (Sarasota, FL, USA). Dithiothreitol (DTT), acrylamide, 1.0 M solution of magnesium chloride, and 1,8-ANS were purchased from SigmaAldrich. Guanidine hydrochloride $(\mathrm{GuHCl})$, and $0.1 \mathrm{M}$ solution of calcium chloride were purchased from Fisher Scientific. The hydrophobic fluorescent probes 2,6-ANS and nile red were purchased from Invitrogen and used without further purification. Nickel nitrilotriacetic acid (Ni-NTA) super flow resin was purchased from Qiagen and diethylaminoethyl Sepharose fast flow resin (DEAE) was purchased from GE Healthcare. 
All other reagents were purchased from Fisher Scientific and Sigma-Aldrich at analytical grade and used without further purification.

\subsection{Methods}

\subsubsection{DREAM protein expression}

The expression vectors of DREAM, DREAM-C, DREAM-D150N, DREAME186Q, DREAM-E234Q, DREAM-KChIP1, and DREAM-NCS1 were transformed into Escherichia coli strain BL21 (Stratagene) by using the heat shock technique for protein expression.

\subsubsection{DREAM protein isolation and purification}

Recombinant DREAM protein isolation and purification were carried out using the method described previously (Osawa et al. 2001; Osawa et al., 2005; Lusin et al., 2008). A single colony of transformed DREAM cells was suspended in $50 \mathrm{~mL}$ of Luria-Bertani medium supplemented with $100 \mathrm{mg} \mathrm{L}^{-1}$ of ampicillin and grown overnight at $37{ }^{\circ} \mathrm{C}$ and 250 RPM in a vigorous shaker (MaxQ 400) for a starter culture. Subsequently, $5 \mathrm{~mL}$ of starter culture were transferred into $1 \mathrm{~L}$ of Terrific Broth medium supplemented with 100 $\mathrm{mg} \mathrm{L}^{-1}$ ampicillin. Cells were incubated at $37^{\circ} \mathrm{C}$ and $250 \mathrm{rpm}$ until the optical density at $600 \mathrm{~nm}\left(\mathrm{OD}_{600}\right)$ reached between 0.8 to 1.0 , then the protein expression was induced by adding $700 \mu \mathrm{M}$ isopropyl 1-thio- $\beta$-D-galactopyranoside (IPTG) to the cell culture. The cells were further incubated at $25^{\circ} \mathrm{C}$ for $16 \mathrm{~h}$ before harvesting by centrifugation at $4{ }^{\circ} \mathrm{C}$ and 10,000 rpm for 10 min (Allegra 64R, Beckman Coulter).

After harvesting, one gram of DREAM wet cells was homogenized in $5 \mathrm{~mL}$ of lysis buffer containing $20 \mathrm{mM}$ Tris-HCl, $\mathrm{pH} 8.0,300 \mathrm{mM} \mathrm{NaCl}, 1 \mathrm{mM} \beta$-mercaptoethanol, $20 \%$ glycerol, 1mM phenylmethyl sulfonyl fluoride (PMSF), 0.2\% Tween 20, $20 \mu \mathrm{g} / \mathrm{mL}$ DNase 
I, $5 \mu \mathrm{g} / \mathrm{mL}$ lysozyme, and $5 \mathrm{mM} \mathrm{MgCl}_{2}$. The suspension was then sonicated with a sonic dismembrator (model 100, Fisher Scientific) for 30 intervals of 20 seconds with 2 min of resting on ice between each interval. The membrane debris and unbroken cells were removed by high spin centrifugation at $4{ }^{\circ} \mathrm{C}$ and 10,000 rpm for $1 \mathrm{~h}$ (Allegra 64R, Beckman Coulter).

The supernatant was collected and loaded into a Ni-NTA super flow column (Qiagen) equilibrated with buffer A containing 20 mM Tris- $\mathrm{HCl}$, pH 8.0, $300 \mathrm{mM} \mathrm{NaCl}, 1 \mathrm{mM} \beta$ mercaptoethanol, and $20 \%$ glycerol. The column was consecutively washed with buffer A containing 5, 10, 20, 30, and $40 \mathrm{mM}$ imidazole until absorbance at $280 \mathrm{~nm}\left(\mathrm{~A}_{280 \mathrm{~nm}}\right)$ was less than 0.03 to remove impurities. DREAM protein was eluted with buffer A containing $250 \mathrm{mM}$ imidazole. Purified DREAM protein was dialyzed overnight in 2 liters of buffer B comprised of $20 \mathrm{mM}$ Tris- $\mathrm{HCl}, \mathrm{pH} 7.4,1 \mathrm{mM}$ EDTA, and $1 \mathrm{mM}$ DTT to remove imidazole and $\mathrm{NaCl}$. The DREAM protein was then applied onto a DEAE column equilibrated with buffer B at $4{ }^{\circ} \mathrm{C}$. The DEAE column was sequentially washed with buffer B containing 20,40,60, and $80 \mathrm{mM} \mathrm{NaCl}$ until $\mathrm{A}_{280 \mathrm{~nm}}$ was below 0.02. Finally, DREAM protein was eluted with $300 \mathrm{mM} \mathrm{NaCl}$ in buffer $\mathrm{B}$. The purity of protein was assessed using SDS-PAGE electrophoresis.

\subsubsection{Protein purity assay}

\subsubsection{Sodium dodecyl sulfate polyacrylamide gel electrophoresis}

Purity of DREAM protein was verified using one-dimensional gel electrophoresis. In sodium dodecyl sulfate polyacrylamide gel electrophoresis (SDS-PAGE) method, the negatively charged sodium dodecyl sulfate (SDS) coasted protein migrates in response to an electrical field created by the negative cathode attached to the upper buffer chamber and 
the positive anode attached to the lower buffer chamber through pores formed by polyacrylamide in the presence of sodium dodecyl sulfate (Gallagher, 2006). The standard continuous SDS-PAGE, in which Tris-tricine was used for both gel and electrode solution, was used in this experiment. Electrophoresis gels containing 4 - $20 \%$ Tris - Glycine gradient were purchased from Lonza (PAGEr ${ }^{\mathrm{TM}}$ Gold Precast Gels). The electrophoresis running buffer and treatment buffer were prepared according to the chemical compositions in Tables 3.1 and Table 3.2, respectively. The samples were prepared by mixing protein solution (about $3 \mathrm{mg} / \mathrm{ml}$ ) with treatment buffer. The samples were then boiled for $10 \mathrm{~min}$ in a water bath. Subsequently, $10 \mu \mathrm{L}$ of the protein mixed with treatment buffer was loaded into each well of the electrophoresis gel. Electrophoresis was carried out at a constant voltage $(120 \mathrm{~V})$ with varying current using a power supply (Model FB300, Fisher Scientific) until the protein samples reached the bottom. The gel was removed and stained in the Coomassie blue solution containing $0.1 \%$ Coomassie blue dye, $50 \%$ methanol, and $10 \%$ acetic acid overnight. The gel was then de-stained in a solution containing $50 \%$ methanol, $10 \%$ acetic acid, and 40\% water for about 3 hours.

Table 3.1: Chemical components for electrophoresis running buffer

\begin{tabular}{|l|c|}
\hline \multicolumn{1}{|c|}{ Chemical components } & Concentration \\
\hline Tris-HCl buffer (pH 8.3) & $0.025 \mathrm{M}$ \\
\hline Glycine & $0.192 \mathrm{M}$ \\
\hline SDS & $0.1 \%$ \\
\hline
\end{tabular}


Table 3.2: Chemical components for sample treatment buffer

\begin{tabular}{|l|c|}
\hline \multicolumn{1}{|c|}{ Chemical components } & Concentration \\
\hline Tris-HCl buffer (pH 6.8) & $0.125 \mathrm{M}$ \\
\hline SDS & $4 \%$ \\
\hline Glycerol & $20 \%$ \\
\hline Bromophenol blue & $0.02 \%$ \\
\hline DTT & $0.2 \mathrm{M}$ \\
\hline
\end{tabular}

\subsubsection{DREAM protein concentration determination}

\subsubsection{Bicinchoninic acid assay (BCA)}

Bicinchoninic acid forms a purple complex with $\mathrm{Cu}^{1+}$ in an alkaline solution, which has a strong absorbance at $562 \mathrm{~nm}$ (Smith et al., 1985). Since amino acid residues such as tryptophan, tyrosine, and cysteine reduce $\mathrm{Cu}^{2+}$ to $\mathrm{Cu}^{1+}$ at $37^{\circ} \mathrm{C}$ (Wiechelman et al., 1988), the concentration of proteins can be determined by monitoring the color produced in the reaction of bicinchoninic acid with $\mathrm{Cu}^{1+}$ (Smith et al., 1985). In this assay, the procedure was followed as described in the BCA protein assay reagent kit (Pierce Biotechnology, Rockford, IL). The standard curve was generated by measuring maximum absorption at $562 \mathrm{~nm}$ for bovine serum albumin standard protein with concentrations varying from 25 $\mu \mathrm{g} / \mathrm{ml}$ to $2,000 \mu \mathrm{g} / \mathrm{ml}$. DREAM protein concentration was then calculated from the calibration curve of BSA. Consequently, the extinction coefficient of DREAM protein at $280 \mathrm{~nm}$ was determined (Table 3.3).

Table 3.3: DREAM protein extinction coefficient at $280 \mathrm{~nm}$

\begin{tabular}{|l|c|}
\hline \multicolumn{1}{|c|}{ Protein } & $\varepsilon_{280 \mathrm{~nm}}\left(\mathrm{M}^{-1} \mathrm{~cm}^{-1}\right)$ \\
\hline DREAM, DREAM-D150N, DREAM-E186Q, & \\
DREAM-E234Q, and DREAM-KChIP1 & 19,000 \\
\hline DREAM-C & 11,000 \\
\hline
\end{tabular}




\subsubsection{Ultraviolet-Visible (UV-Vis) spectroscopy assay}

Since aromatic amino acid residues such as phenylalanine, tyrosine, and tryptophan in protein absorb light with an absorbance maxima ranging from $260 \mathrm{~nm}$ to $280 \mathrm{~nm}$, protein concentration can be quickly determined using the Beer-Lambert law (equation 3.1) when the protein extinction coefficient is known.

$$
A=-\log \left(\frac{I}{I_{0}}\right)=\varepsilon \cdot l \cdot C
$$

In this expression, $\mathrm{A}$ is absorption, $\mathrm{I}_{0}$ and $\mathrm{I}$ are light intensity before and after transmitted through a sample, which has concentration, $\mathrm{C}$, in unit of $\mathrm{M}$; path length, $l$, in unit of $\mathrm{cm}$; and the molar extinction coefficient, $\varepsilon$, in unit of $\mathrm{M}^{-1} \mathrm{~cm}^{-1}$.

\subsubsection{Fluorescence spectroscopy}

Fluorescence spectroscopy has been widely used in many applications in biochemistry and biophysics research. The advantage of this technique is its high sensitivity, which allows the detection of signals at low concentration ranging from $\mathrm{nM}$ to $\mu \mathrm{M}$ (Boens et al., 2007; Lakowicz, 2010).

\subsubsection{The light absorption and emission of fluorophores}

The process of light absorption and emission of fluorophores are often described by a simple electronic state diagram, known as Jablonski diagram, as shown in Figure 3.1 (Lakowicz, 2006; Periasamy and Clegg, 2010).

After light is absorbed at a particular wavelength, fluorophores in an illuminated volume of the sample undergo an instantaneous electronic transition from the singlet ground state $\left(\mathrm{S}_{0}\right)$ into the singlet of the first $\left(\mathrm{S}_{1}\right)$ or second $\left(\mathrm{S}_{2}\right)$ state. The transition time of 
the light absorption process is too short $\left(10^{-15} \mathrm{~s}\right)$ for significant displacement of the nuclei, a phenomenon known as the Frank-Codon principle (Lakowicz, 2006). At room temperature, since the thermal energy is not adequate to populate higher vibrational levels, light absorption typically occurs from the lowest vibrational level of the ground state.

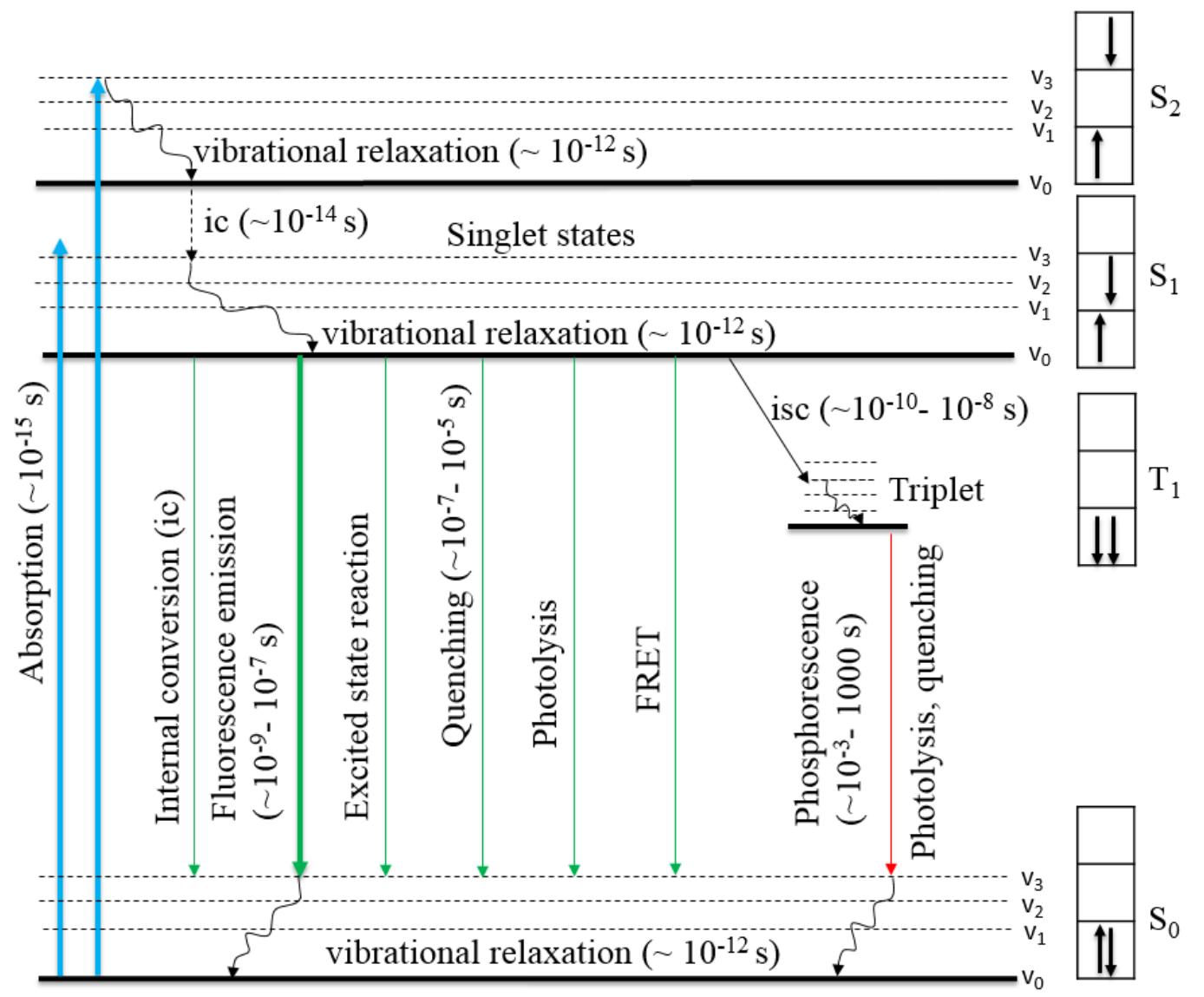

Figure 3.1 The Perrin-Jablonski diagram. The arrows represent the transition of a quantum change. The transitions begin at the lowest vibrational levels of each electronic state $\left(\mathrm{S}_{0}\right.$, $S_{1}$, and $S_{2}$ ) for both absorption (blue arrows) and de-excitation (green arrows). The transition from the excited state to the ground state always happens from the first lowest level of $\mathrm{S}_{1}$. The non-emissive de-excitation transitions are internal conversion (ic), intersystem crossing (isc), excited state reaction, quenching, photolysis, and FRET. The excited molecule passes from the singlet $\left(\mathrm{S}_{1}\right)$ to the triplet $\left(\mathrm{T}_{1}\right)$ state via isc. The emission from $\mathrm{T}_{1}$ is phosphorescence (red arrow). The other de-excitation transitions from $\mathrm{T}_{1}$ are similar to that of the $S_{1}$. Adapted from (Lakowicz 2006; Periasamy and Clegg 2010). 
In general, fluorophores are usually excited to some higher vibrational levels ( $\mathrm{v}_{1}$, $\mathrm{v}_{2}$, or $\left.\mathrm{v}_{3}\right)$; however, they quickly relax to the lowest vibrational level ( $\left.\mathrm{v}_{\mathrm{o}}\right)$ of each electronic state via the thermal relaxation pathway. In addition, the excited fluorophores in the $S_{2}$ state also quickly relax to the $\mathrm{v}_{0}$ of $\mathrm{S}_{1}$ state in less than $10^{-14} \mathrm{~s}$ via an internal conversion (IC) and vibrational relaxation pathways (Figure 3.1). Therefore, the transition from the excited state to the ground state of fluorophores always occurs from the $v_{0}$ level of $S_{1}$ state. When promoted to the $v_{0}$ level of $S_{1}$ state, fluorophores undergo fast vibration relaxation before returning to the singlet ground state through either radiative pathway (emission of a photon, $k_{r}$ ) or non-radiative pathways including vibrational relaxation, and internal conversion $\left(k_{i c}\right)$. With a very low probability, the excited fluorophores can pass to the triplet state $\left(\mathrm{T}_{1}\right)$ via the intersystem crossing process $\left(k_{i s c}\right)$; however, transition from $\mathrm{T}_{1}$ to $\mathrm{S}_{0}$ state is forbidden (Figure 3.1). In the presence of quenching molecules, the excited fluorophores may also be dynamically or statically quenched by surrounding quenchers $\left(k_{q}\right)$ (Lakowicz, 2006).

\subsubsection{Characteristics of fluorescence emission}

\subsection{Fluorescence emission spectra}

The interesting process of de-excitation transitions is the emission of photons. The emission spectrum is represented by a plot of fluorescence intensity as a function of emission wavelength. One important characteristics of the emissive process is that fluorescence emission occurs at lower energies (or longer wavelengths) relative to its absorption energy, known as the Stokes shift phenomenon. In addition, the same emission spectrum is observed irrespective of its excitation wavelengths, also known as Kasha's

rule. However, the shape and maxima of an emission spectrum also depend upon molecular 
relaxation of the solvent and energy transfer to quenchers surrounding the fluorophore's environment (Lakowicz, 2006).

\subsection{Fluorescence quantum yield}

The quantum yield $(\Phi)$ of fluorophores provides information about the efficiency of the fluorescence process. Fluorescence quantum yield can be defined by the fraction of rate constants of the radiative emission of a photon $\left(k_{r}\right)$ over the total rate constants of both radiative and non-radiative processes, including internal conversion $\left(k_{i c}\right)$ and intersystem crossing $\left(k_{i s c}\right)$ as shown in equation 3.2 (Lakowicz, 2006).

$$
\Phi=\frac{k_{r}}{k_{r}+k_{i c}+k_{i s c}}
$$

\subsection{Quantum yield measurements}

Fluorescence quantum yield can be calculated by using either an absolute or a relative method (Wurth et al., 2013). Quantum yield experiments performed in this dissertation project were determined using the relative method described by Williams et al. (Williams et al., 1983). The method involves the use of a well characterized standard sample with known quantum yield $\left(\Phi_{\mathrm{ST}}\right)$ and the quantum yield of testing sample $\left(\Phi_{\mathrm{x}}\right)$ is calculated by equation 3.3.

$$
\Phi_{X}=\Phi_{S T}\left(\frac{\operatorname{Grad}_{X}}{\operatorname{Grad}_{S T}}\right)\left(\frac{\eta_{X}^{2}}{\eta_{S T}^{2}}\right)
$$

In this expression, Grad is the gradient from the plot of integrated fluorescence intensity versus absorbance, and $\eta$ is the refractive index of the solvent. Subscript $x$ and st denote the testing and standard samples, respectively. 


\subsection{Fluorescence lifetime}

Fluorescence lifetime is defined as the average time that the fluorophore resides in its electronic excited state following excitation (Lakowicz, 2006). Thus, fluorescence lifetime $(\tau)$ of a fluorophore is the reciprocal of the sum of radiative and non-radiative rate constants as shown in equation 3.4.

$$
\tau=\frac{1}{k_{r}+k_{i s}+k_{i s c}}
$$

Fluorescence lifetime is important for biophysical studies because lifetime of a fluorophore provides useful molecular information about structural fluctuation and conformational heterogeneity as a result of structural perturbations near the fluorophore in the investigating system (Valeur, 2002; Lakowicz, 2006; Periasamy, 2010). Fluorescence lifetime measurements can be performed by either time-domain (TD) or frequency-domain (FD), also known as phase-modulation approach. In the TD approach, the fluorophore is excited with a short laser pulse and fluorescence emission intensity is recorded as a function of time. On the other hand, in the FD approach, when a fluorophore is excited with a modulated sinusoidally light source at high frequencies, the lifetime of the fluorophore causes the emission to be delayed in time, called phase shift $(\phi)$, and a decrease in the peak-to-peak height, also known as modulation, with respect to that of modulated excitation (Figure 3.2) (Gratton et al. 1984; Lakowicz, 2006; Ross and Jameson 2008). The phase shift is measured from the zero-crossing times of the modulated components, whereas modulation (M) is the ratio of signal amplitude (AC) at modulation frequency, $\omega$, to the average signal (DC) (Figure 3.2) (Gratton et al., 1984; Ross and Jameson, 2008, Lakowicz, 2006). Since throughout my dissertation project fluorescence lifetime 
measurements were performed with a FD fluorimeter, a brief discussion about the theory and data analysis of FD fluorescence lifetimes is introduced to give insight into the significance of fluorescence lifetimes of the studies in this project.

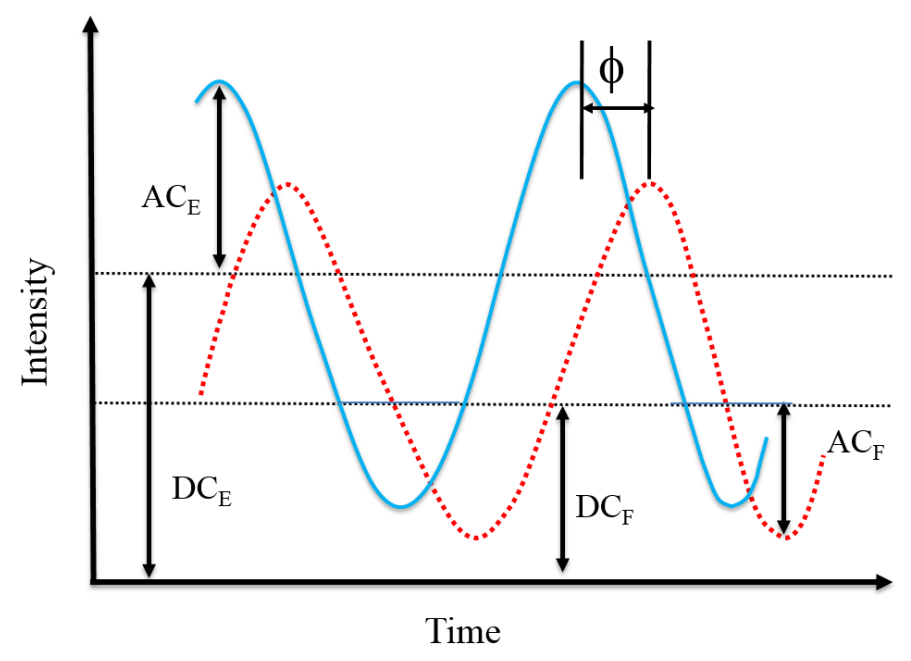

Figure 3.2: Relative phase shift $(\phi)$ and modulation of the excitation (subscript E) and emission light (subscript F) in FD fluorescence spectroscopy. AC and DC indicate the amplitude and offset of the respective waves. Adapted from (Ross and Jameson, 2008).

\subsection{Basic principles of frequency-domain lifetime}

\section{measurements}

For a sample containing $\mathrm{n}$ fluorophores, each of which has the same probability of emitting in a given period of time, the excitation intensity and the fluorescence emission intensity are described by equations 3.5 and 3.6, respectively (Ross and Jameson, 2008).

$$
\begin{aligned}
& E(t)=E_{0}\left[1+M_{E} \sin (\omega t)\right] \\
& F(t)=F_{0}\left[1+M_{F} \sin (\omega t+\phi)\right]
\end{aligned}
$$

In these expressions, $\mathrm{E}(\mathrm{t})$ and $\mathrm{E}_{0}$ are the excitation intensities, $\mathrm{F}(\mathrm{t})$ and $\mathrm{F}_{0}$ are the fluorescence emission intensities at time $t$ and time zero, respectively, $\phi$ is the phase shift 
between the excitation and emission, and $\omega$ is the angular modulation frequency equal to $2 \pi$ of the linear frequency $f$ (in radian/s). The modulation of excitation $\left(\mathrm{M}_{\mathrm{E}}\right)$ and the modulation of emission $\left(\mathrm{M}_{\mathrm{F}}\right)$ are described by equations 3.7, 3.8, and 3.9.

$$
\begin{gathered}
M_{F}=\frac{A C_{F}}{D C_{F}} \\
M_{E}=\frac{A C_{E}}{D C_{E}} \\
M=\frac{M_{F}}{M_{E}}
\end{gathered}
$$

In these expressions, $\mathrm{AC}$ and $\mathrm{DC}$ are signal amplitude and the average signal at modulation frequency, $\omega$, respectively. The subscripts $\mathrm{E}$ and $\mathrm{F}$ indicate excitation and fluorescence emission, respectively. $\mathrm{M}$ is the relative modulation of the emission and excitation modulations.

The characteristic phase lifetime $\left(\tau_{\phi}\right)$ and modulation lifetime $\left(\tau_{\mathrm{M}}\right)$ at any single modulation frequency can be calculated by using equations 3.10 and 3.11 (Ross and Jameson 2008).

$$
\begin{aligned}
\tau_{\phi} & =\omega^{-1} \tan \phi \\
\tau_{M} & =\frac{1}{\omega}\left(\frac{1}{M^{2}}-1\right)^{\frac{1}{2}}
\end{aligned}
$$


These phases and modulation lifetimes are equal $\left(\tau_{\phi}=\tau_{\mathrm{M}}\right)$ only if the intensity decay is a single exponential. However, most samples of interest display more than one lifetime, which cannot be resolved by using equations 3.10 and 3.11. In this case, the lifetimes $\left(\tau_{\phi}\right.$ and $\left.\tau_{M}\right)$ measured at a particular frequency are the result of the weighting various components in the emission (Lakowicz, 2006). In addition, the phase lifetime is shorter than the modulation lifetime $\left(\tau_{\phi}<\tau_{M}\right)$, and both $\tau_{\phi}$ and $\tau_{M}$ decrease at higher modulation frequencies (Lakowicz, 2006).

For a multi-exponential decay system, the phase and modulation values have to be measured in a wide range of modulation frequencies using a variable-frequency instrument. Alternatively, the phase and modulation values can be expressed in the waveforms of sine (S) and cosine $(\mathrm{G})$ functions as shown in equations 3.12 and 3.13 (Ross and Jameson, 2008):

$$
\begin{aligned}
& S=\sum_{i} f_{i} M_{i} \sin \phi_{i} \\
& G=\sum_{i} f_{i} M_{i} \cos \phi_{i}
\end{aligned}
$$

The parameters $\phi_{i}$ and $\mathrm{M}_{i}$ are described in equations 3.14 and 3.15, respectively. Parameter $f_{i}$ is the fractional intensity of the $i^{\text {th }}$ components $\left(\Sigma f_{i}=1\right)$.

$$
\begin{aligned}
& \phi=\tan ^{-1}\left(\frac{S}{G}\right) \\
& M=\left(S^{2}+G^{2}\right)^{\frac{1}{2}}
\end{aligned}
$$


The functions $\mathrm{S}$ and $\mathrm{G}$ are sine and cosine transforms of the impulse response function according to equations 3.14 and 3.15 (Ross and Jameson, 2008). These expressions can be used for any intensity decay law such as Gaussian distribution or discrete distribution and can also be transformed numerically into expressions 3.16 and 3.17 (Lakowicz, 2006).

$$
\begin{array}{r}
S=\frac{\sum_{i} \frac{\alpha_{i} \omega \tau_{i}^{2}}{\left(1+\omega^{2} \tau_{i}^{2}\right)}}{\sum_{i} \alpha_{i} \tau_{i}} \\
G=\frac{\sum_{i} \frac{\alpha_{i} \tau_{i}}{\left(1+\omega^{2} \tau_{i}^{2}\right)}}{\sum_{i} \alpha_{i} \tau_{i}}
\end{array}
$$

In these above expressions, $\alpha_{i}$ corresponds to the pre-exponential decay factor, $\tau_{i}$ represents the individual lifetimes, and $\sum_{i} \alpha_{i} \tau_{i}$ is proportional to the steady-state intensity. The relationship between $\alpha_{\mathrm{i}}$ and $f_{i}$ in equations 3.16 and 3.17 is given by expression 3.18, assuming that the quantum yield of emitting species are proportional to their lifetimes (Lakowicz, 2006; Ross and Jameson, 2008).

$$
f_{i}=\frac{\alpha_{i} \tau_{i}}{\sum_{j} \alpha_{j} \tau_{j}}
$$

In the least-square analysis, the parameters $\alpha_{i}$ and $\tau_{i}$ are varied to yield the best fit between the measured data and calculated values justified by a minimum value for the reduced chi-squared value $\left(\chi^{2}\right)$ in 3.19 (Lakowicz, 2006). 


$$
\chi^{2}=\frac{1}{v} \sum\left[\frac{\phi_{m}-\phi_{c}}{\delta \phi}\right]^{2}+\frac{1}{v} \sum\left[\frac{M_{m}-M_{c}}{\delta M}\right]^{2}
$$

In this expression, $\phi$ and $M$ correspond to the phase and modulation values, respectively, the subscripts $c$ and $m$ indicate the calculated and measured values, and $\delta \phi$ and $\delta \mathrm{M}$ are the standard deviations of each phase and modulation measurement, respectively. The value $v$ is the number of degrees of freedom, which is equal to twice the number of frequencies minus the number of variable parameters. The best fit between the calculated values and the experimental values are typically characterized by a $\chi^{2}$ value close to 1 (Lakowicz, 2006). In the frequency-domain approach, Fluorescence lifetime data are often described in terms of the contributions of various components to the emission or fractional intensities, $f_{i}$, which correspond to the contribution of the $\mathrm{i}^{\text {th }}$ component to the photocurrent. On the other hand, the pre-exponential, $\alpha_{i}$, is usually reported in the time-domain approach (Ross and Jameson, 2008).

\subsection{Fluorescence quenching}

Fluorescence quenching provides useful information on the accessibility of the fluorophore to the solvent. The accessible process depends not only on the structure of the molecules surrounding the fluorophore, but also on the fluorophore charged environment (Jameson, 2014). The quenching process can occur via dynamic quenching (also known as collisional quenching), static quenching, or simultaneous dynamic and static quenching (Jameson, 2014). 


\subsection{Collisional quenching}

Collisional quenching occurs when the excited fluorophore comes in contact with an atom or molecule that can facilitate the non-radiative transitions to the ground state (Valeur, 2002). Thus, fluorescence quantum yield and fluorescence lifetime of a single exponential system in the presence of quenchers are given by equations 3.20 and 3.21 (Valeur, 2002).

$$
\begin{aligned}
& \Phi=\frac{k_{r}}{k_{r}+k_{i s}+k_{i s c}+k_{q}[Q]}=\frac{k_{r}}{1 / \tau_{0}+k_{q}[Q]} \\
& \frac{\tau_{0}}{\tau}=1+k_{q}[Q]
\end{aligned}
$$

In these expressions, $k_{q}$ is the rate of quenching, [Q] is the quencher concentration, and $\tau_{0}$ and $\tau$ are lifetimes in the absence and presence of quenchers, respectively. Other parameters have the same meaning as described previously.

Since quantum yield is proportional to fluorescence emission intensity, the combination of equations 3.20 and 3.21 gives the Stern-Volmer equation (Valeur, 2002).

$$
\frac{\Phi_{0}}{\Phi}=\frac{I_{0}}{I}=1+k_{q} \tau_{0}[Q]=1+K_{S V}[Q]
$$

In this expression, $\mathrm{I}_{0}$ and $\mathrm{I}$ are steady-state fluorescence intensities in the absence and presence of quencher $\mathrm{Q}$, respectively. The Stern-Volmer constant $\mathrm{K}_{\mathrm{sv}}$ is equal to $\mathrm{k}_{\mathrm{q}} \tau_{0}$. Thus, the bimolecular quenching constant $\left(k_{q}\right)$ can be calculated from $\mathrm{K}_{\mathrm{sv}}$ and determined from the plot of $\mathrm{I}_{0} / \mathrm{I}$ against the quencher concentration $[\mathrm{Q}]$ when the excited-state lifetime in the absence of quencher $\left(\tau_{0}\right)$ is known (Valeur, 2002). 


\subsection{Static quenching}

Static quenching occurs when a fluorophore interacts with a quencher to form a non-fluorescent complex in the ground state. Upon light absorption, the complex rapidly returns to the ground state without emission of a photon. Thus, the steady-state fluorescence intensity decreases upon addition of Q, but lifetime of the fluorophore is unaffected (Lakowicz, 2006; Valeur, 2002). The static quenching can be described by equation 3.23.

$$
\frac{I_{0}}{I}=1+K_{s v}[Q]
$$

The linear dependence of $\mathrm{I}_{0} / \mathrm{I}$ in the expression for static quenching (equation 3.23) is identical to that of dynamic quenching in equation 3.22, except that the quenching constant $\left(\mathrm{K}_{\mathrm{sv}}\right)$ is the association constant.

\subsubsection{Fluorescence anisotropy}

Fluorescence anisotropy measurement provides useful information on molecular mobility, size, shape, and flexibility of molecules, fluidity of a medium, and other parameters (Valeur, 2002).

\subsection{Basic principles and theory of fluorescence anisotropy}

In a homogenous solution, fluorophores are randomly oriented in the ground state. When these fluorophores are excited with vertically polarized light, whose electric vector has transition moments oriented along the z-axis (Figure 3.3), the intensity of emission can be measured through a polarizer. If the emission polarizer is oriented parallel $(\|)$ to the zaxis, the observed intensity is called $\mathrm{I}_{\| \cdot}$. Likewise, if the polarizer is perpendicular $(\perp)$ to $\mathrm{z}$ - 
axis, the observed intensity is called $\mathrm{I}_{\perp}$. The anisotropy $(\mathrm{r})$ is calculated from these $\mathrm{I}_{\|}$and $\mathrm{I}_{\perp}$ intensity components by using equation 3.24 (Lakowicz, 2006).

$$
r=\frac{I_{\|}-I_{\perp}}{I_{\|}+2 I_{\perp}}
$$

Since the intensity difference between parallel and perpendicular components $\left(I_{\|}-I_{\perp}\right)$ is normalized by the total intensity $\left(I_{\|}+2 I_{\perp}\right)$, anisotropy is independent of the fluorophore concentration (Lakowicz, 2006).

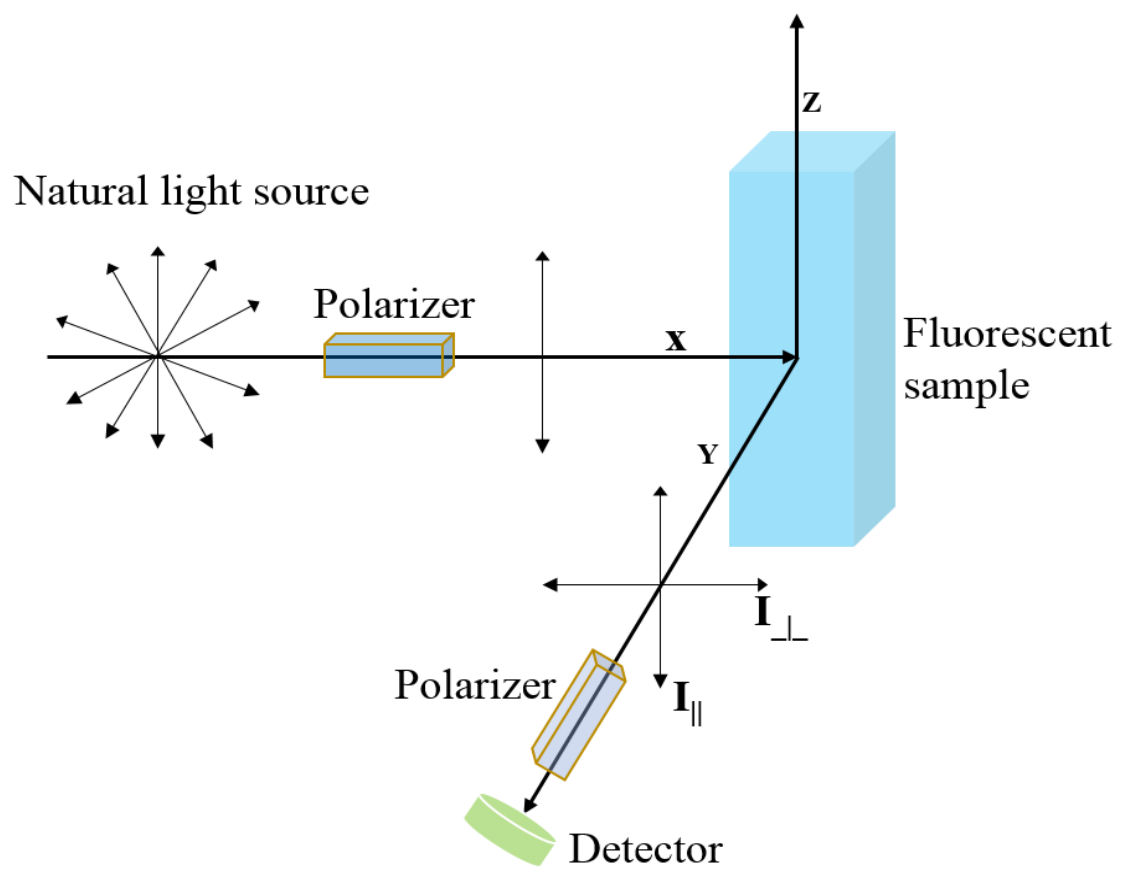

Figure 3.3: Schematic diagram for measurement of fluorescence anisotropy. Adapted from (Lakowicz, 2006).

Upon excitation by polarized light, a fluorophore, whose dipole is oriented with angles $\theta$ and $\phi$ respectively relative to the z-axis and $y$-axis as shown in Figure 3.4, creates the electric field (E), described by equation 3.25. 


$$
E(\theta, \phi)=k \frac{\sin \theta}{r} \hat{\theta}
$$

In this expression, $\mathrm{k}$ is a constant, $\mathrm{r}$ is the distance from the fluorophore, and $\hat{\boldsymbol{\theta}}$ is the unit vector along the $\theta$ coordinate.

The intensity of emission of that fluorophore is proportional to the square of the electric field, described by equation 3.26 (Lakowicz, 2006).

$$
I(\theta, \phi)=k^{2} \frac{\sin ^{2} \theta}{r^{2}} \hat{r}
$$

where $\hat{r}$ is a unit vector in the direction of propagation.

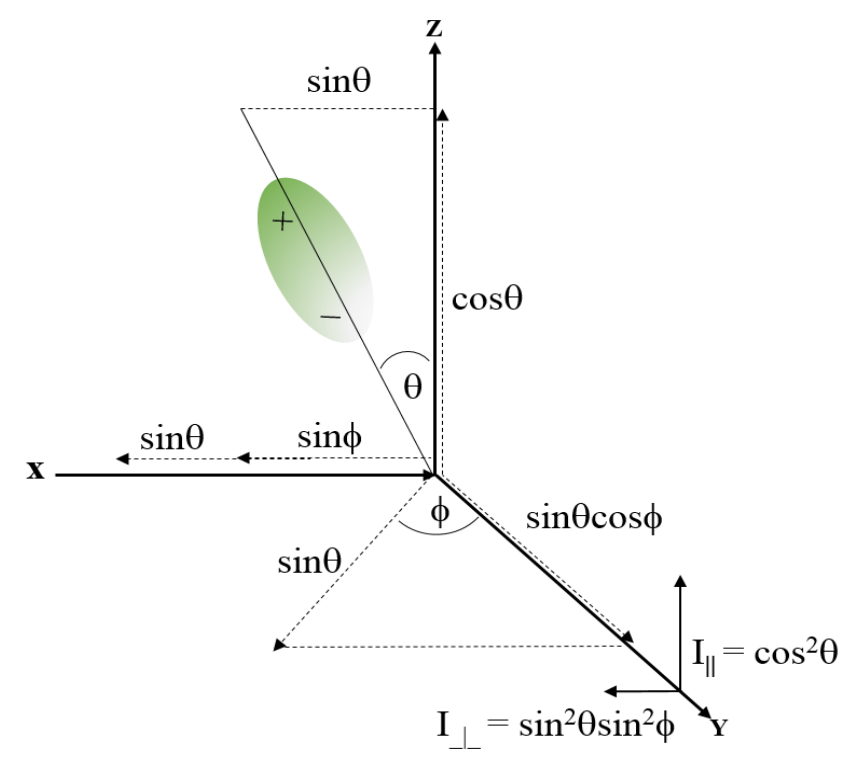

Figure 3.4: Schematic diagram illustrating emission intensities for a single fluorophore in a coordinate system. Adapted from (Lakowicz, 2006).

Since the electric field points in the same direction as the emission transition moment, the projection of the field onto the z-axis is proportional to $\cos \theta$ and the intensity is proportional to $\cos ^{2} \theta$. Likewise, the field along the $\mathrm{x}$-axis is proportional to $\sin \theta \sin \phi$ and 
the intensity is proportional to $\sin ^{2} \theta \sin ^{2} \phi$. Hence, $I_{\|}$and $I_{\perp}$ components can be expressed by equations 3.27 and 3.28 (Lakowicz, 2006).

$$
\begin{aligned}
& I_{\|}(\theta, \phi)=\cos ^{2} \theta \\
& I_{\perp}(\theta, \phi)=\sin ^{2} \theta \sin ^{2} \phi
\end{aligned}
$$

Because all the excited fluorophores at angle $\phi$ from the $y$-axis are symmetrically distributed around the z-axis, the population of excited fluorophores is oriented with values of $\phi$ from 0 to $2 \pi$ with equal probability. The average value of $\sin ^{2} \phi$ is given by equation 3.29 .

$$
\left\langle\sin ^{2} \phi\right\rangle=\frac{\int_{0}^{2 \pi} \sin ^{2} \phi d \phi}{\int_{0}^{2 \pi} d \phi}=\frac{1}{2}
$$

Therefore, equation 3.28 can be written as equation 3.30 .

$$
I_{\perp}(\theta, \phi)=\frac{1}{2} \sin ^{2} \theta
$$

Assuming that the probability of a collection of fluorophores, oriented at an angle between $\theta$ and $\theta+d \theta$ relative to z-axis, is $f(\theta)$, then, $\mathrm{I}_{\|}$and $\mathrm{I}_{\perp}$ can be rewritten by equations 3.31 and 3.32 .

$$
\begin{aligned}
& I_{\|}=\int_{0}^{\pi / 2} f(\theta) \cos ^{2} \theta d \theta=k\left\langle\cos ^{2} \theta\right\rangle \\
& I_{\perp}=\frac{1}{2} \int_{0}^{\pi / 2} f(\theta) \sin ^{2} \theta d \theta=\frac{k}{2}\left\langle\sin ^{2} \theta\right\rangle
\end{aligned}
$$

where $\mathrm{k}$ is an instrumental constant.

Using $\sin ^{2} \theta=1-\cos ^{2} \theta$, equation 3.32 can be rewritten by equation 3.33. 


$$
r=\frac{3\left\langle\cos ^{2} \theta\right\rangle-1}{2}
$$

In this expression, the anisotropy is determined by the average value of $\cos ^{2} \theta$, where $\theta$ is the angle of the emission dipole relative to the z-axis (Lakowicz, 2006).

\subsection{Steady-state anisotropy}

The steady-state anisotropy can be calculated from an average of the anisotropy decay $\mathrm{r}(\mathrm{t})$ over the intensity decay $\mathrm{I}(\mathrm{t})$ by using equation 3.34 (Lakowicz, 2006).

$$
r=\frac{\int_{0}^{\infty} I(t) r(t) d t}{\int_{0}^{\infty} I(t) d t}
$$

For a single-exponential intensity decay with lifetime, $\tau$, the steady-state anisotropy is given by equation 3.35 .

$$
r=\frac{r_{0}}{1+\tau / \theta}
$$

In this expression, $\mathrm{r}_{0}$ is the limiting anisotropy and $\theta$ is the angle of the emission dipole relative to the z-axis.

\subsection{Frequency-domain anisotropy decay}

Frequency-domain (FD) anisotropy decay is also known as the dynamic polarization method (Jameson, 2014). Anisotropy decay provides information on rotational modalities of a fluorophore as a function of time. Thus, it allows us to investigate the

dynamics of the system such as size, shape, and flexibility (Lakowicz, 2006; Ross and Jameson, 2008). 


\subsection{Theory of FD anisotropy decay}

In FD anisotropy decay, a fluorophore is illuminated with vertically polarized, modulated light, and the parallel and perpendicular emission intensities are observed through a rotatable polarizer. The observable quantities for the anisotropy decay measurements are the phase shift $\left(\Delta_{\omega}\right)$ between perpendicular $\left(\phi_{\perp}\right)$ and parallel $\left(\phi_{\|}\right)$ emission components at the modulation frequency $(\omega)$ and the ratio of the modulated amplitudes of the parallel $\left(\mathrm{M}_{\|}\right)$and perpendicular $\left(\mathrm{M}_{\perp}\right)$ components of emission (Figure 3.5) (Lakowicz, 2002).

The changes of $\Delta_{\omega}$ and $\Lambda_{\omega}$ are because of the rotation of the excited fluorophore during the excited state lifetime, which changes the orientation of the emission dipole of the fluorophore (Ross and Jameson, 2008). The relation between the observed $\Delta_{\omega}$ and $\Lambda_{\omega}$ with respect to rotational parameters of fluorophores in a single rotating spherical system is displayed in equations 3.36 and 3.37 (Weber, 1977; Ross and Jameson, 2008).

$$
\begin{aligned}
& \Delta_{\omega}=\tan ^{-1}\left[\frac{18 \omega r_{0} R}{\left(k^{2}+\omega^{2}\right)\left(1+r_{0}-2 r_{0}^{2}\right)+6 R\left(6 R+2 k+k r_{0}\right)}\right] \\
& \Lambda_{\omega}=\left[\frac{\left(\left(\left(1-r_{0}\right)+6 R\right)^{2}+\left(1-r_{0}\right)^{2} \omega^{2}\right)}{\left[\left(1+2 r_{0}\right) k+6 R\right]^{2}+\left(1+2 r_{0}\right)^{2} \omega^{2}}\right]^{\frac{1}{2}}
\end{aligned}
$$

In these expressions, $\mathrm{r}_{0}$ is the limiting anisotropy, which is observed in the absence of rotation, $\mathrm{k}$ is the radiative rate constant $(1 / \tau)$ of the fluorophore, and $\mathrm{R}$ is the rotational 
diffusion coefficient, which is equal to twice the reciprocal of the rotational relaxation time ( $\rho$ ), where $\rho$ is equal three times the rotational correlation time (Ross and Jameson, 2008).

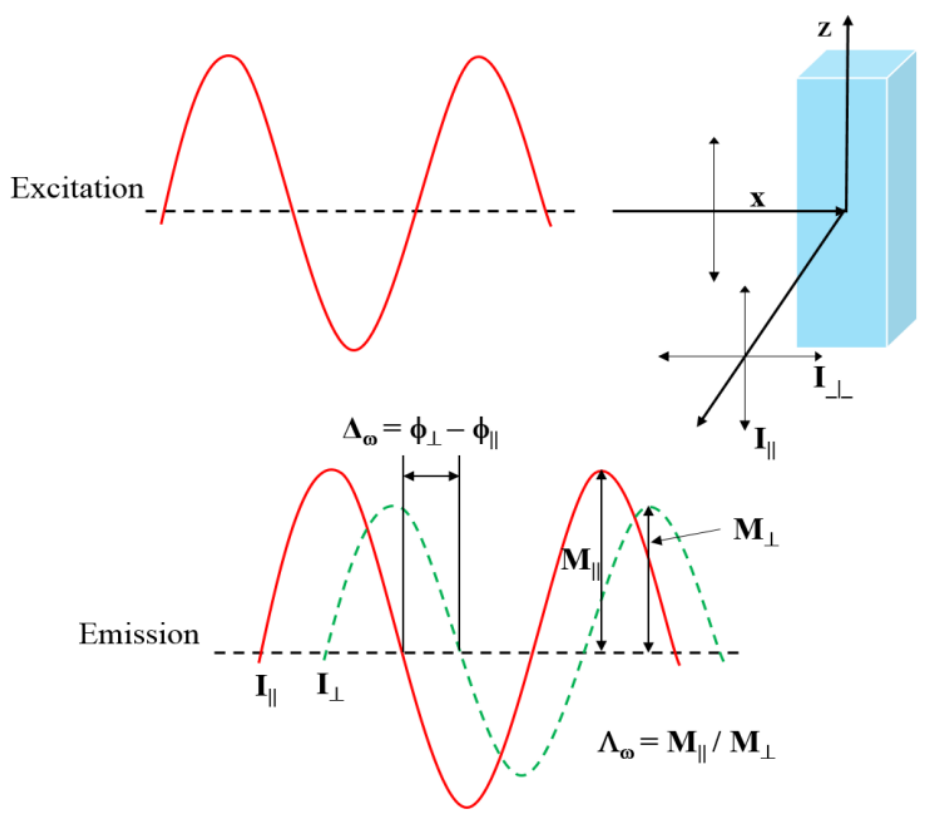

Figure 3.5: Schematic diagram for measurement of anisotropy decays with frequencydomain. Adapted from (Lakowicz, 2002).

\subsection{Data analysis of anisotropy decay}

In FD anisotropy decay approach, the phase $\operatorname{shift}\left(\Delta_{\mathrm{c} \omega}\right)$ and modulation ratio $\left(\Lambda_{\mathrm{c} \omega}\right)$ are measured using equations 3.38 and 3.39 .

$$
\begin{aligned}
& \Delta_{c \omega}=\tan ^{-1}\left(\frac{D_{\|} N_{\perp}-N_{\|} D_{\perp}}{N_{\|} N_{\perp}+D_{\|} D_{\perp}}\right) \\
& \Lambda_{c \omega}=\left(\frac{N_{\|}^{2}+D_{\|}^{2}}{N_{\perp}^{2}+D_{\perp}^{2}}\right)^{\frac{1}{2}}
\end{aligned}
$$


In these expressions, parameters $\mathrm{D}$ and $\mathrm{N}$ are expressions for the sine and cosine transforms of the individual polarized decays as shown in equations 3.40 and 3.41 (Weber, 1977; Lakowicz, 2006).

$$
\begin{aligned}
& N_{k}=\int_{0}^{\infty} I_{k}(t) \sin \omega t d t \\
& D_{k}=\int_{0}^{\infty} I_{k}(t) \cos \omega t d t
\end{aligned}
$$

where $\mathrm{k}$ indicates the perpendicular and parallel orientations.

Thus, anisotropy decay parameters can be obtained by minimizing the squared deviations between measured and calculated values using equation 3.42 .

$$
\mathrm{X}^{2}=\frac{1}{v} \sum_{\omega}\left(\frac{\Delta_{\omega}-\Delta_{c \omega}}{\delta \Delta}\right)^{2}+\frac{1}{v} \sum_{\omega}\left(\frac{\Lambda_{\omega}-\Lambda_{c \omega}}{\delta \Lambda}\right)^{2}
$$

where $\delta \Delta$ and $\delta \Lambda$ are the uncertainties in the differential phase and modulation ratio, respectively (Joseph R. Lakowicz 2006).

\subsection{Anisotropy decay fitting models}

Anisotropy decay models are varied since the size, shape, and flexibility of the fluorophore, and its local environment can affect anisotropy decays of the investigated system. A spherical molecule displays a single rotational correlation time. However, the anisotropy decay for a non-spherical fluorophore is more complicated because there are different rotational rates around each axis (Lakowicz, 2006). Several anisotropy decay models routinely used for data analysis throughout my dissertation project are described 
below. For the first case of a spherical system, the decay of anisotropy with time, $\mathrm{I}(\mathrm{t})$, is given by 3.43 (Jameson, 2014).

$$
r=\frac{I_{\|}-I_{\perp}}{I_{\|}+2 I_{\perp}}=r_{0} e^{-t / \theta}
$$

In the case of a mixture of large and small particles, anisotropy decay reflects the sum of exponential components as displayed in equation 3.44a (Jameson, 2014).

$$
r(t)=r_{1} e^{-t / \theta_{1}}+r_{2} e^{-t / \theta_{2}}
$$

In the case of a local rotation of a probe attached to a spherical particle, the decay anisotropy is given by equation 3.44 b (Jameson, 2014).

$$
r(t)=r_{1} e^{-t / \theta_{1}}+r_{2} e^{-\left(t / \theta_{1}+t / \theta_{2}\right)}
$$

In these expressions, $r_{1}$ and $r_{2}$ are anisotropy amplitudes, $\theta_{1}$ and $\theta_{2}$ are respectively the rotational correlation time of fast (local) motion and slow (global) motion of a mixed system.

The experimentally determined rotational correlation times can be compared to expected values $\left(\theta_{\text {calc }}\right)$ for a spherical protein calculated by using the Stokes-Einstein equation 3.45 (Lakowicz, 2006).

$$
\theta_{\text {calc }}=\frac{\eta V}{R T}
$$

where $\eta$ is the viscosity of water $(\eta=1.02 \mathrm{cP})$ (Korson et al., 1969), $\mathrm{V}$ is the hydrodynamic molecular volume, $\mathrm{R}$ is the ideal gas constant, and $\mathrm{T}$ is the temperature $\left(20^{\circ} \mathrm{C}\right)$. 


\subsubsection{Fluorescence stopped-flow kinetics}

\subsection{Basic principles of fluorescence stopped-flow kinetics}

Fluorescence stopped-flow kinetics is a useful method that directly measures the kinetics of a bi-molecular reaction. Kinetics parameters provide reaction rates, which can be used to investigate mechanisms and binding constants (Gibson, 1969; Hargrove, 2005). In this method, small volumes of protein and ligand, such as a short peptide, are rapidly driven from syringes A and B through valves A and B into an observation mixer chamber as shown in Figure 3.6.

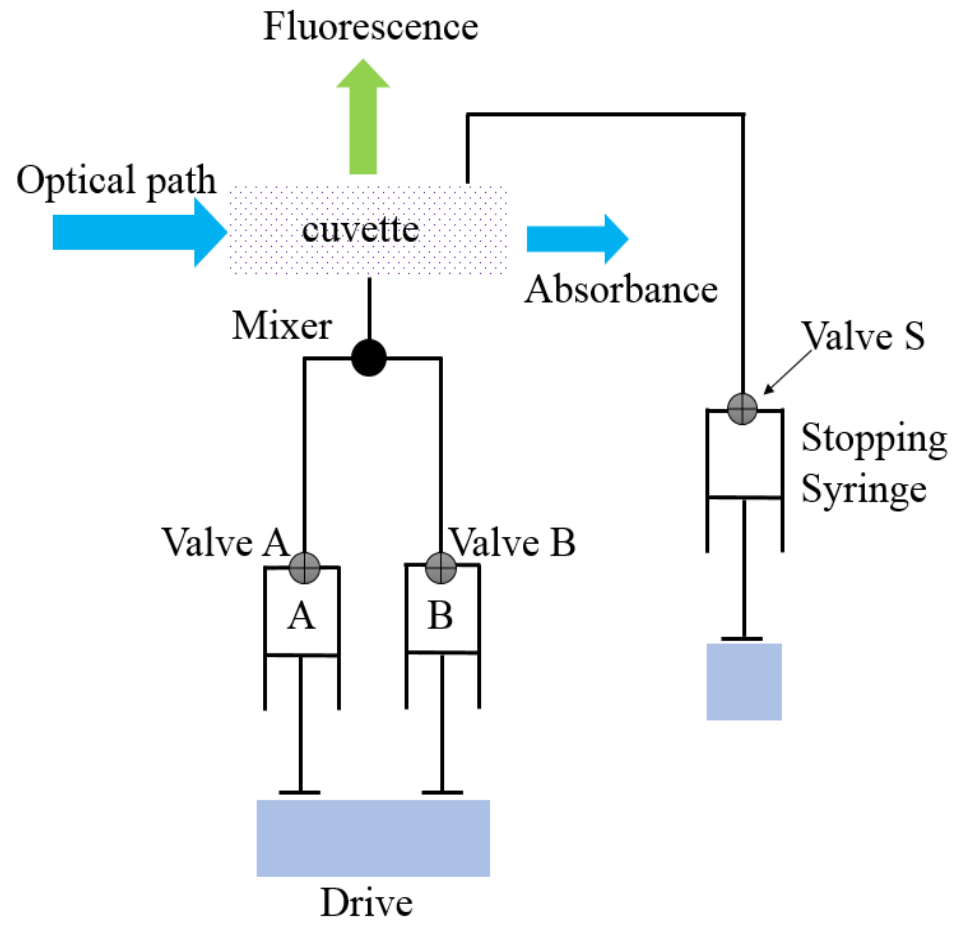

Figure 3.6: Schematic diagram of a stopped-flow mixer. Reaction A and B are driven into the mixer through valves A and B. The previous content of the tube and cuvette are flushed out the stopping syringe via valve $S$ that is open during flow. When the plunger of the stopping syringe hits the block, the valve $S$ is closed and the contents of the cuvette can be probed during reaction mixture. Adapted from (Hargrove, 2005). 
The resultant reaction volume displaces the content of an observation cuvette and thus fills it with freshly mixed reagents. As long as the stopping syringe is filled with old reaction solution, the plunger hits a block causing the flow to be stopped instantaneously (Figure 3.6). The time during which the solution enters the observation cuvette is known as the dead time of a stopped-flow system, which is less than $8 \mathrm{~ms}$ for a fast kinetic stoppedflow system, model SFA-20 (TgK Scientific).

\subsection{Fluorescence stop-flow kinetics measurements and data analysis}

The kinetics measurements were performed with a ChronosFD spectrofluorometer (ISS, Champaign, IL) coupled to a SFA-20 rapid stopped-flow apparatus (TgK Scientific, Bradford-on-Avon, UK). Kinetics data were analyzed using single and double exponential decay models (Origin 8 software, OriginLab Corp. Northampton, MA).

\subsubsection{Fluorophores}

Fluorophores are classified into intrinsic and extrinsic probes. The intrinsic fluorophores are natural aromatic amino acid residues in proteins including tryptophan, tyrosine, and phenylalanine. On the other hand, extrinsic fluorophores are fluorescence emissive compounds such as FITC, DNS-Cl, ethidium bromide (EtBr), or ANS. Since many proteins and DNA of interest are nonfluorescent, extrinsic fluorophores are frequently labeled on the proteins or DNA to create a fluorescent system. The fluorophores, routinely used in this project, are listed in Table 3.4. 
Table 3.4: List of extinction coefficients $\left(\varepsilon \lambda_{\mathrm{nm}}\right)$, emission maximum wavelength $\left(\right.$ Em. $\left.\lambda_{\max }\right)$ of fluorophores used.

\begin{tabular}{|l|c|c|c|l|}
\hline \multicolumn{1}{|c|}{ Chemical } & $\begin{array}{c}\varepsilon_{\lambda \mathrm{nm}} \\
\left(\mathrm{M}^{-1} \mathrm{~cm}^{-1}\right)\end{array}$ & Solvent & References & \multicolumn{1}{|c|}{ Manufactures } \\
\hline 1,8-ANS & $\begin{array}{c}7,800 \\
\left(\varepsilon_{372 \mathrm{~nm}}\right)\end{array}$ & Methanol & Diwu et al., 1997 & Sigma \\
\hline 2,6 -ANS & $\begin{array}{c}27,000 \\
\left(\varepsilon_{319 \mathrm{~nm})}\right.\end{array}$ & Methanol & Diwu et al., 1997 & Molecular Probes \\
\hline Nile red & $\begin{array}{c}19,600 \\
\left(\varepsilon_{552 \mathrm{~nm}}\right)\end{array}$ & DMSO & Castro et al., 2005 & Molecular Probes \\
\hline $\begin{array}{l}\text { Fluorescein } \\
\text { isothyocyanate (FITC) }\end{array}$ & $\begin{array}{c}75,000 \\
\left(\varepsilon_{493 \mathrm{~nm}}\right)\end{array}$ & Water & Jobbágy et al., 1973 & Molecular Probes \\
\hline $\begin{array}{l}\text { Ethidium bromide } \\
\text { (EtBr) }\end{array}$ & $\begin{array}{c}5,680 \\
\left(\varepsilon_{478 \mathrm{~nm})}\right)\end{array}$ & Water & Garbett et al. 2004 & Thermal Fisher \\
\hline
\end{tabular}

\subsubsection{Circular dichroism spectroscopy}

Circular dichroism (CD) is an excellent method to evaluate the secondary structure, folding, and binding properties of proteins because CD measurements can be performed with low concentration of protein $(<20 \mu \mathrm{g} / \mathrm{ml})$ in a physiological buffer within a few hours (Greenfield, 2006). The principle, applications, and analysis of CD data have been intensively described by Greenfield (Greenfield, 2004; Greenfield, 2004; Greenfield, 2006; Greenfield, 2006; Greenfield, 2006; Greenfield, 2015). Therefore, the basic principles of CD spectroscopy and its applications in evaluating the secondary structure of the DREAM protein as well as protein stability will be concisely highlighted in the next sections.

\subsubsection{Basic principles of circular dichroism}

A beam of light consists of electric and magnetic fields which oscillate in all planes perpendicular to the propagating direction. Linearly polarized light, generated by passing the light through a prism, has an electric field (E) that vibrates sinusoidally in a single plane 
(Kuball, 1999). If linearly polarized light passes through a birefringent material, the electric field will be separated into two components of equal magnitude.

Superposition of these two sinusoidal waves with a phase-shift equal to a quarter of its wavelength $(\lambda / 4)$ creates circular polarized waves, whose electric field rotates circularly about their propagating direction while the vector retains constant magnitude (Figure 3.7) (Berova et al., 2000). If observed from the front, the circularly polarized light can be seen as the result of two vectors of equal length: one rotating clockwise (right handed, $E_{R}$ ) and the other rotating counter-clockwise (left handed, $E_{L}$ ) as shown in Figure 3.7 (Greenfield, 2006).

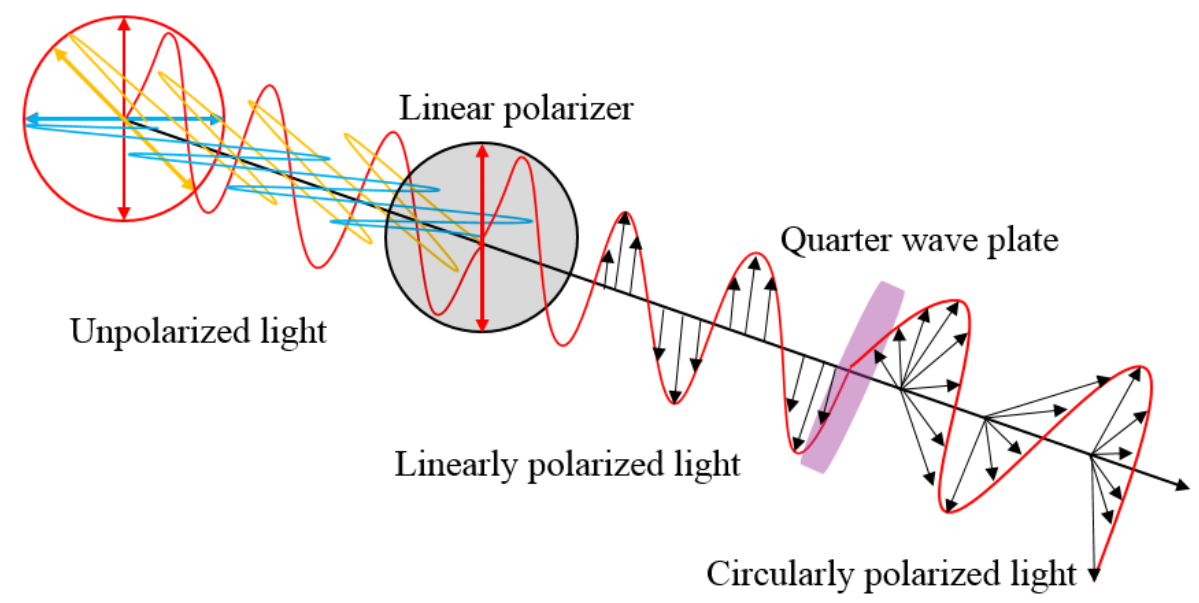

Figure 3.7: Scheme representing linearly polarized light that is converted using a linear polarizer and the circularly polarized light that is created using a quarter-wave $(\lambda / 4)$ plate. Adapted from (Berova et al., 2000).

If the circular polarized light passes through an isotropic environment (achiral samples), it will not experience any difference as shown in Figure 3.8A. However, when circular polarized light passes through chiral samples such as amino acids (except glycine), the $E_{L}$ and $E_{R}$ components are absorbed differently, resulting in an elliptical polarization as shown in Figure 3.8B (Kelly et al., 2005). 
The unequal absorption $(\Delta \mathrm{A})$ of the $\mathrm{E}_{\mathrm{L}}$ and $\mathrm{E}_{\mathrm{R}}$ components of circular polarized light by chiral samples is known as $\mathrm{CD}$. The $\mathrm{CD}$ instrument measures the difference in absorbance $(\Delta A)$ between $E_{L}$ and $E_{R}$ components of circular polarized light $\left(A_{L}-A_{R}\right)$ described in equation 3.46 (Berova et al., 2000; Kelly et al., 2005).

$$
\Delta A=A_{L}-A_{R}=\left(\varepsilon_{L}-\varepsilon_{R}\right) \cdot l \cdot C=\Delta \varepsilon \cdot l \cdot C
$$

In this expression, parameters $\varepsilon_{\mathrm{L}}$ and $\varepsilon_{\mathrm{R}}$ are extinction coefficients of the sample for $\mathrm{E}_{\mathrm{L}}$ and $E_{\mathrm{R}}$ components, respectively. $C$ is concentration of the sample, and $l$ is the path length of the optical cell.

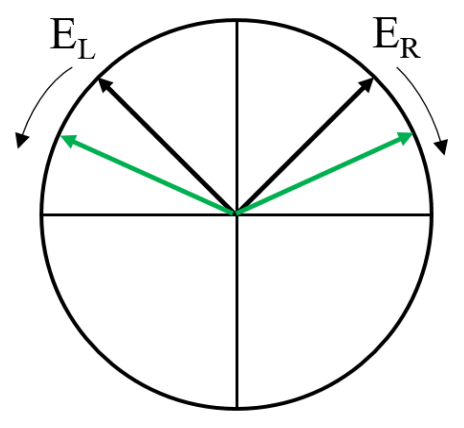

A

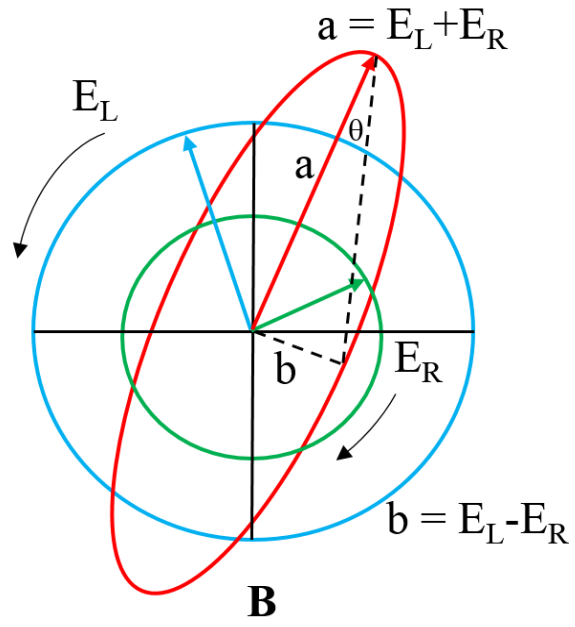

B

Figure 3.8: A: when circularly polarized light passes through achiral samples, its $E_{L}$ and $E_{\mathrm{R}}$ components of are constant. B: when circularly polarized light passes through chiral samples, $\mathrm{E}_{\mathrm{L}}$ (blue) and $\mathrm{E}_{\mathrm{R}}$ (green) components are absorbed in distinctly, resulting in elliptical polarized light (red). Adapted from (Kelly et al., 2005).

The unit of CD is reported in degrees of ellipticity $(\theta)$, whose tangent is the ratio of the minor (b) to the major (a) axis of ellipse as shown in Figure 3.8B, as a function of wavelength (Berova et al., 2000). 


$$
\theta=32.98(\triangle A)
$$

The molar ellipticity $([\theta])$ in $\operatorname{deg} \mathrm{cm}^{2} \mathrm{dmol}^{-1}$ is converted using equation 3.48 (Berova et al., 2000).

$$
[\theta]=\frac{100(\Delta A)}{C \cdot l}
$$

In measurements of proteins or peptides, the molar concentration unit is calculated using mean residue weight (MRW), which is the molar weight of the protein divided by the number of amino acid residues per molecule. Thus, the residue ellipticity $\left([\theta]_{\mathrm{MR}}\right)$ can be reported in the unit of degrees $\mathrm{cm}^{2} \mathrm{dmol}^{-1}$ by equation 3.49 , where $\mathrm{C}$ is concentration expressed in $\mathrm{mg} \mathrm{ml}^{-1}$ (Berova et al., 2000).

$$
[\theta]_{M R}=\frac{\theta \times(M R W)}{10 \times C \times l}
$$

\subsubsection{Information from CD studies of proteins}

Analysis of a CD spectrum in the far and near UV regions provides information about the proteins' secondary and tertiary structures. Chromophores of interest in proteins widely absorb circularly polarized light ranging from $190 \mathrm{~nm}$ to $650 \mathrm{~nm}$ (Berova et al., 2000).

Information on the secondary structure of a protein can be characterized using a far UV CD spectrum (between $190 \mathrm{~nm}$ and $250 \mathrm{~nm}$ ). For instance, $\alpha$-helical structure shows a strong peak centered at $192 \mathrm{~nm}$ according to perpendicular $\pi \rightarrow \pi^{*}$ transition and intensively negative peaks centered at $208 \mathrm{~nm}$ and $222 \mathrm{~nm}$ according to parallel $\pi \rightarrow \pi^{*}$ transition and $n \rightarrow \pi^{*}$ transition, respectively (Figure 3.9). On the other hand, $\beta$-sheet 
displays a positive peak at $196 \mathrm{~nm}$ according to $n \rightarrow \pi^{*}$ transition and a negative peak at $218 \mathrm{~nm}$ according to $\pi \rightarrow \pi^{*}$ transition. The random coil shows a positive peak at $212 \mathrm{~nm}$ and $195 \mathrm{~nm}$ according to the transitions of $\pi \rightarrow \pi^{*}$, and $n \rightarrow \pi^{*}$, respectively (Figure 3.9) (Berova et al., 2000).

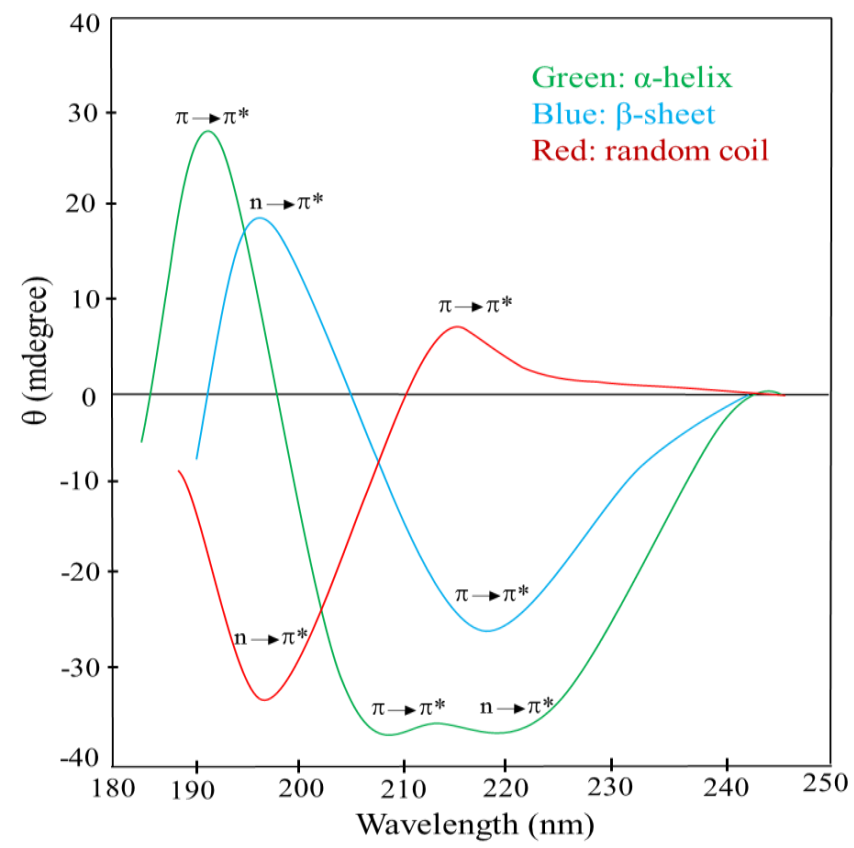

Figure 3.9: Far UV CD spectra associated with various types of secondary structure of proteins. Adapted from (Berova et al., 2000).

Aromatic side chains of phenylalanine, tyrosine, tryptophan, and disulphide bonds absorb circular polarized light near the UV region ranging from $260 \mathrm{~nm}$ to $320 \mathrm{~nm}$. A near UV CD spectrum reflects the micro environment of aromatic amino acid side chains such as the types of aromatic amino acids present, their mobility, the nature of their environment, and their spatial disposition in the protein (Kelly et al., 2005). For instance, tryptophan shows a fine peak between $290 \mathrm{~nm}$ and $305 \mathrm{~nm}$, while tyrosine displays a broad peak between $275 \mathrm{~nm}$ and $282 \mathrm{~nm}$ and a shoulder at $285 \mathrm{~nm}$. Phenylalanine shows a sharp band between $255 \mathrm{~nm}$ and $270 \mathrm{~nm}$ (Figure 3.10), whereas a disulphile bond has a weak and 
broad peak centered at $260 \mathrm{~nm}$ (Kelly et al., 2005). However, the actual shape and magnitude of the near UV CD spectrum of a protein containing aromatic side chains depends on the nature of their environment as previously mentioned (Kelly et al., 2005).

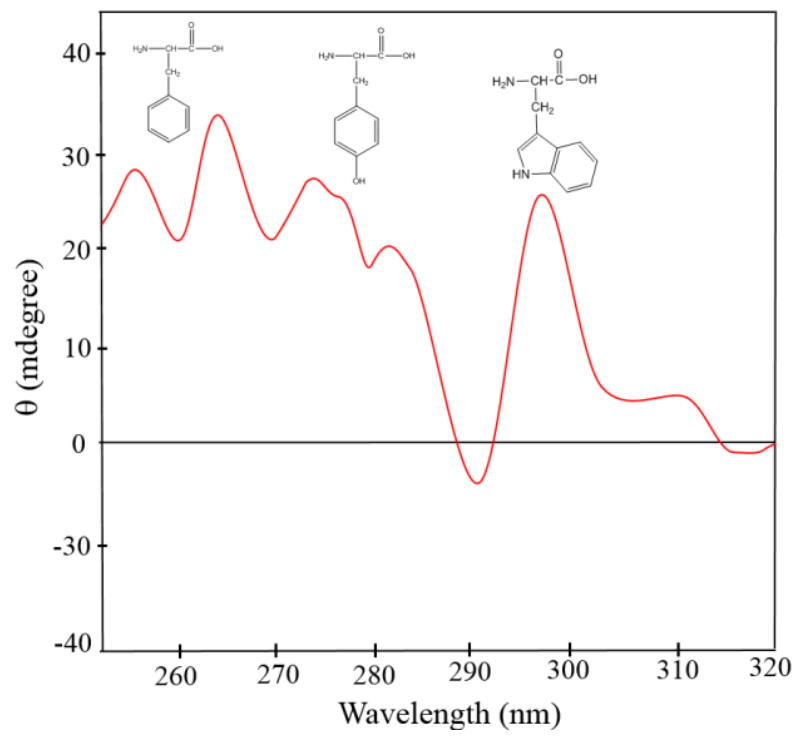

Figure 3.10: Near UV CD spectrum for various aromatic side chains of proteins. Adapted from (Kelly, Jess et al. 2005).

Furthermore, cofactors such as heme and flavin only display a strong CD signal when associated with protein partners. Thus, the CD signals in the appropriate spectral region are excellent probes for cofactor association (Kelly et al., 2005). For instance, a CD spectrum of hemeproteins shows a strong peak centered around $410 \mathrm{~nm}$ with other bands in range from 350 to $650 \mathrm{~nm}$ depending on spin state and coordination of the heme iron (Kelly et al., 2005).

\subsubsection{CD measurements of DREAM protein}

The CD measurements of DREAM protein were performed on a JASCO J-810 CD spectropolarimeter (JASCO Inc. Easton, MD). A sample containing $40 \mu \mathrm{M}$ of purified DREAM protein was placed in a quartz cuvette with $0.1 \mathrm{~cm}$ path length. The far UV CD 
spectra were collected at $20 \mathrm{~nm} / \mathrm{min}$ scan rate from 190 to $260 \mathrm{~nm}$ with $1 \mathrm{~nm}$ band width, 8 seconds response time, $1 \mathrm{~nm}$ data pitch, and 5 accumulations. The baseline was acquired in the same way with buffer solution and the CD spectra of protein were corrected by subtracting from the baseline. All the $\mathrm{CD}$ spectra were recorded at $16^{\circ} \mathrm{C}$.

\subsubsection{Sample preparation for CD measurements}

To investigate $\mathrm{Mg}^{2+}$ and/or $\mathrm{Ca}^{2+}$ induced conformational changes in DREAM protein, $\mathrm{Ca}^{2+}$ and $\mathrm{Ca}^{2+} \mathrm{Mg}^{2+}$ bound DREAM samples were prepared by adding $\mathrm{CaCl}_{2}$ or $\mathrm{MgCl}_{2}$ solutions to protein samples to a final concentration of $1 \mathrm{mM} \mathrm{Ca}^{2+}$ or $5 \mathrm{mM} \mathrm{Mg}^{2+}$. Apo and $\mathrm{Mg}^{2+}$ DREAM and DREAM-C samples were prepared by adding $1 \mathrm{mM}$ EDTA and $1 \mathrm{mM}$ EGTA with $5 \mathrm{mM} \mathrm{MgCl} 2$ to protein solutions, respectively. To minimize the difference on $\mathrm{CD}$ spectra of $\mathrm{Apo}, \mathrm{Mg}^{2+}, \mathrm{Ca}^{2+}$, and $\mathrm{Ca}^{2+} \mathrm{Mg}^{2+}$ bound forms due to the mismatch of protein concentration, four samples with identical protein concentration (40 $\mu \mathrm{M})$ were prepared from an initial stock protein which was split into individual sample and EDTA, EGTA, $\mathrm{Mg}^{2+}$, or $\mathrm{Ca}^{2+}$ was added in equal volumes.

Table 3.5: Volumes and concentrations of protein and chemicals used to prepare CD samples of Apo, $\mathrm{Mg}^{2+}, \mathrm{Ca}^{2+}$, and $\mathrm{Ca}^{2+} \mathrm{Mg}^{2+}$ bound DREAM with identical concentration of DREAM protein

\begin{tabular}{|c|c|c|c|c|c|c|}
\hline $\begin{array}{l}\text { DREAM } \\
\text { Samples }\end{array}$ & $\begin{array}{c}\text { Vol. of } \\
500 \mu \mathrm{M} \\
\text { DREAM } \\
(\mu L)\end{array}$ & $\begin{array}{c}\text { Vol. of } \\
0.1 \mathrm{M} \\
\text { EDTA } \\
(\mu L)\end{array}$ & $\begin{array}{c}\text { Vol. of } \\
0.1 \mathrm{M} \\
\text { EGTA } \\
(\mu L)\end{array}$ & $\begin{array}{c}\text { Vol. of } \\
0.5 \mathrm{M} \\
\mathrm{MgCl}_{2} \\
(\mu L)\end{array}$ & $\begin{array}{c}\text { Vol. of } \\
0.1 \mathrm{M} \\
\mathrm{CaCl}_{2} \\
(\mu L)\end{array}$ & $\begin{array}{l}\text { Vol. of } \\
\text { CD buffer } \\
(\mu L)\end{array}$ \\
\hline Apo & 32 & 4 & & & & 364 \\
\hline $\mathrm{Mg}^{2+}$ & 32 & & 4 & 4 & & 360 \\
\hline $\mathrm{Ca}^{2+}$ & 32 & & & & 4 & 364 \\
\hline $\mathrm{Ca}^{2+} \mathrm{Mg}^{2+}$ & 32 & & & 4 & 4 & 360 \\
\hline
\end{tabular}


The buffer used for CD experiments contains $20 \mathrm{mM}$ Tris-HCl pH 7.4, $1 \mathrm{mM}$ DTT, and 10 mM LDAO freshly prepared in decalcified water. The concentrations and volumes of stock DREAM protein, EDTA, EGTA, $\mathrm{CaCl}_{2}, \mathrm{MgCl}_{2}$, and $\mathrm{CD}$ buffer are listed in Table 3.5.

\subsubsection{Isothermal titration calorimetry (ITC)}

\subsubsection{Principle of ITC}

The technical details and the principle of isothermal titration calorimetry have been described elsewhere (McKinnon et al., 1984; Jelesarov and Bosshard 1999; VelazquezCampoy et al., 2004; Privalov and Ebrary, 2012). Briefly, consider the simplest binding reaction between a macromolecule $(\mathrm{M})$ which has a single binding site for the ligand $(\mathrm{L})$. The association constant $\left(\mathrm{K}_{\mathrm{a}}\right)$ is defined as:

$$
K_{a}=\frac{[M L]}{[M] \times[L]}
$$

In this expression, $[\mathrm{ML}],[\mathrm{M}]$, and $[\mathrm{L}]$ are the corresponding concentrations.

The thermodynamic relationship between the association constant $K_{a}$ and Gibbs energy of association $\Delta \mathrm{G}$ can be expressed in terms of enthalpy $(\Delta H)$ and entropy $(\Delta S)$ change in the process displayed by equation 3.51 .

$$
\Delta G=-R T \ln K_{a}=\Delta H-T \Delta S
$$

In this expression, $\mathrm{R}$ is the gas constant $\left(1.9872 \mathrm{cal} \mathrm{K}^{-1} \mathrm{~mol}^{-1}\right)$ and $\mathrm{T}$ is the absolute temperature (in Kelvin).

Isothermal Titration Calorimetry is the only method that directly determines all the thermodynamics parameters $\mathrm{n}$ (binding stoichiometry), $\mathrm{K}_{\mathrm{a}}$, and $\Delta \mathrm{H}$ associated with binding reaction in a single experiment. In addition, by performing the same experiment at several 
temperatures and constant pressure, the heat capacity, $\mathrm{C}$, can be obtained from the slope of enthalpy versus temperature plot.

Thermodynamic experiments were performed with VP-ITC MicroCalorimeter (MicroCal LLC, Northampton MA). ITC measures heat released or absorbed by the binding reaction of liquid samples. The VP-ITC instrument contains two identical coin shaped reference and sample cells which are accessible from the top and enclosed in an adiabatic outer shield as shown in Figure 3.11 (Ladbury and Chowdhry, 1996; Ladbury, 2004).

The reference cell is loaded with water or buffer and the sample cell is filled with macromolecule solution (DREAM protein or DNA). These two cells are in contact with the thermal reservoir and initially the temperature in both cells is maintained constant by an electronic power source. Any change in temperature $(\Delta T)$ between the reference and the sample cells results from either more or less heat being added to the sample cell which is balanced by the power feedback mechanism. During operation, the precise amounts of reactants (ligand) are titrated into the sample cell via the rotating microsyringe.

An injection of ligand (DREAM) into the macromolecule (DNA) sample results in the release (exothermic) or absorption (endothermic) of heat within the sample cell which causes a respectively negative or positive change in the power difference (DP) between reference and sample cells. Since DP has units of power $\left(\mathrm{Js}^{-1}\right)$, the time integral of the peak of each injection gives thermal energy $(\Delta \mathrm{H})$ (Ladbury and Chowdhry, 1996; Ladbury, 2004). The ITC data are then analyzed using fitting models in Origin 7 software to calculate binding stoichiometry (n), $\mathrm{K}_{\mathrm{a}}, \Delta \mathrm{H}$, and $\Delta \mathrm{S}$ (MicroCal, LLC, Northampton, USA). 


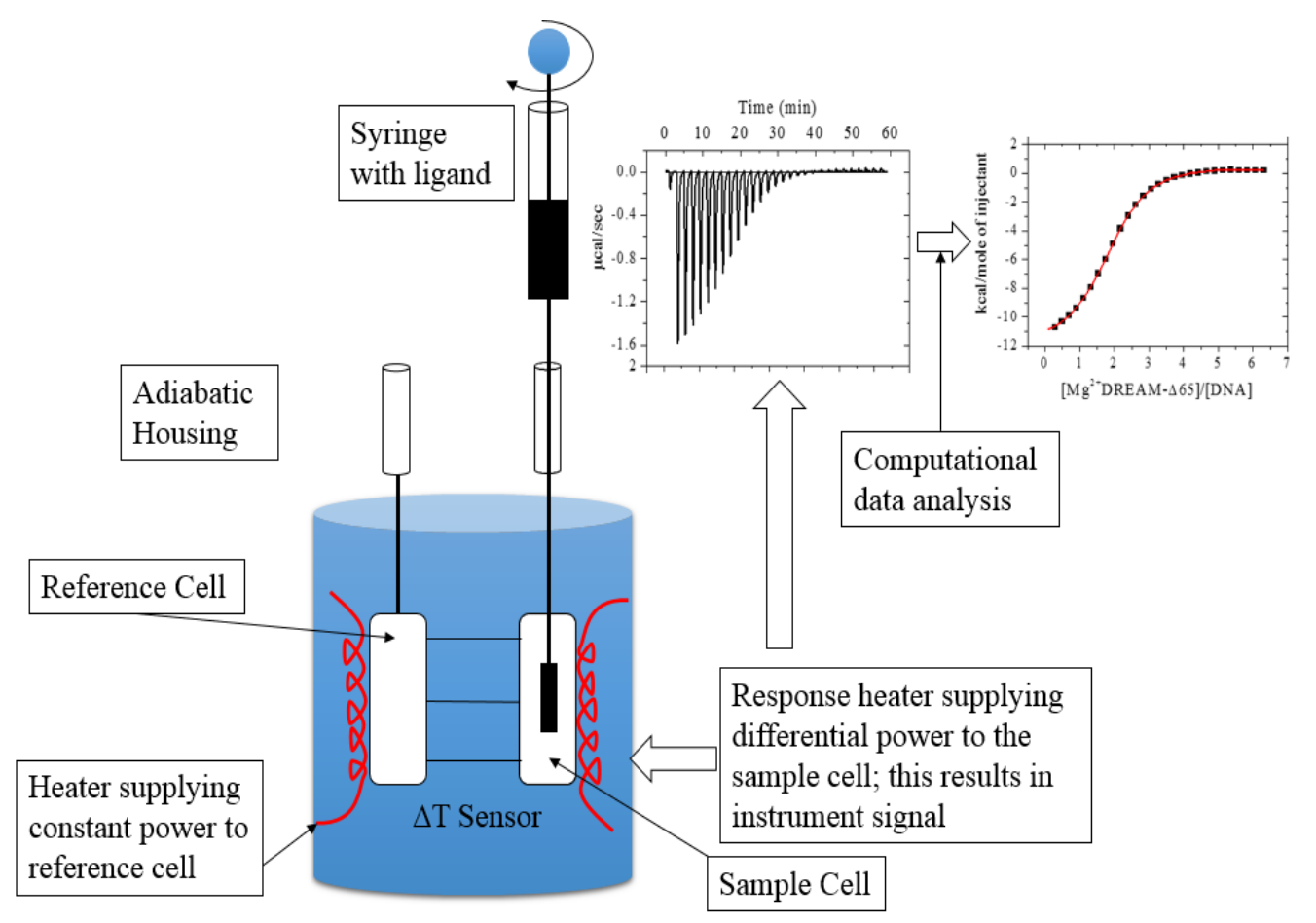

Figure 3.11: Schematic representation of an ITC instrument. The sample cell and the reference cell are kept at the same temperature. The reference cell is always kept at the experimental temperature. The ligand component of the interaction is placed in the syringe and the other in the cell. Upon the first titration (injection), the change in heat associated with binding (endothermic or exothermic) results in a change in temperature in the sample cell. A change in power (heat/s) is required to return the cells to identical temperatures $(\mathrm{T})$ $(\Delta \mathrm{T}=0)$. This change in power is recorded during injections. The binding isotherm and the integrated thermogram are shown in the top right. Adapted from (Ladbury and Chowdhry, 1996; Ladbury, 2004).

\subsubsection{ITC data analysis}

The heat released or absorbed (q) associated with each change of state after each injection is measured. The heat, $\mathrm{q}_{\mathrm{i}}$, is proportional to the increment in the concentration of the complex $[\mathrm{ML}]$ (the parameters $[\mathrm{ML}], \mathrm{M}$, and L were defined previously in equation 3.50) in the calorimetric cell after each injection, $i$, as shown in equation 3.52 .

$$
q_{i}=V \Delta H_{a}\left([M L]_{i}-[M L]_{i-1}\right)
$$


In this expression, $\Delta \mathrm{H}_{\mathrm{a}}$ is the enthalpy of binding and $V$ is the calorimetric cell volume. Equation 3.52 is used for a single titration (injection). However, an actual ITC experiment is done with series of injections. Thus, equation 3.53a is used.

$$
q_{i}=\Delta H(T) V\left[M_{T}\right]\left(\frac{K_{a}[L]_{i}}{1+K_{a}[L]_{i}}-\frac{K_{a}[L]_{i-1}}{1+K_{a}[L]_{i-1}}\right)
$$

The isothermal parameters $n, K_{a}$, and $\Delta H_{a}$ are directly obtained from the fitting curve of an isotherm using equation $3.53 \mathrm{~b}$ because the total concentration of ligand ([L]) and the ratio of total ligand relative to the total protein concentration ([M]) are known (Figure 3.12).

$$
[L]_{i}=\frac{\left[L_{T}\right]-\left[M_{T}\right]-1 / K_{a} \pm \sqrt{\left(\left[L_{T}\right]-\left[M_{T}\right]-1 / K_{a}\right)^{2}-4\left[L_{T}\right]}}{2}
$$

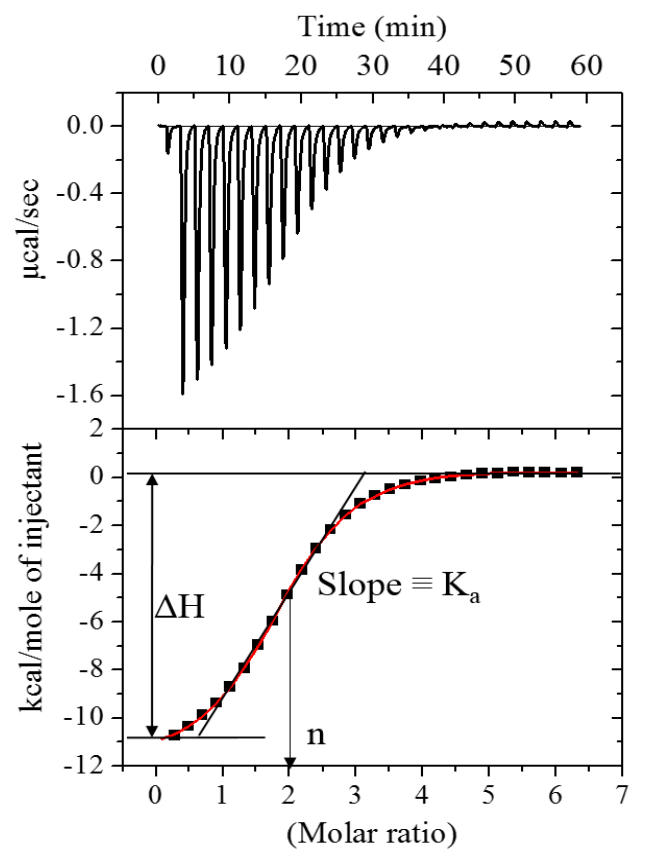

Figure 3.12: A schematic diagram illustrating isothermal parameters being generated from an isotherm fitting curve. 


\subsection{Methods used in chapter 4}

\subsubsection{Steady-state fluorescence measurements}

Steady-state emission spectra were measured using a PC1 fluorimeter (ISS, Champaign, IL). For steady-state emission measurements, $40 \mu \mathrm{M}$ DREAM in $20 \mathrm{mM}$ Tris buffer $\mathrm{pH}$ 7.4, $1 \mathrm{mM}$ DTT and $10 \mathrm{mM} \mathrm{N}, \mathrm{N}$-dimethyldodecylamine $\mathrm{N}$-oxide (LDAO) was placed in a $0.2 \times 1.0 \mathrm{~cm}$ quartz cell and emission spectra were recorded using $295 \mathrm{~nm}$ excitation. The spectra were corrected for the inner filter effect and the quantum yield for W169 emission was calculated using equation 3.3 in section 3.2.5.2.2.1, and the quantum yield of tryptophan $(\Phi=0.13$ ) as a standard (Lakowicz, 2006).

\subsubsection{Fluorescence quenching}

Quenching studies were performed by measuring W169 fluorescence emission intensity in the range from $300 \mathrm{~nm}$ to $450 \mathrm{~nm}$ using an excitation wavelength of $295 \mathrm{~nm}$, after addition of small aliquots of freshly prepared $10 \mathrm{M}$ acrylamide solution following 5 min incubation. The decrease in the emission intensity at $340 \mathrm{~nm}$ and $335 \mathrm{~nm}$ for the $\mathrm{Ca}^{2+}$ free and $\mathrm{Ca}^{2+}$ bound form of DREAM, respectively, was corrected for the inner filter effect. The data were analyzed using the Stern-Volmer equation (equation 3.22 in section 3.2.5.2.4.1). Before determination of the Stern-Volmer constant, the emission spectra were corrected for an inner filter effect as described above (Stegemeyer, 1969; Puchalski et al., 1991).

\subsubsection{Fluorescence lifetime measurements}

The fluorescence lifetime measurements were performed on a ChronoFD fluorimeter (ISS, Illinois-Champaign). The output of a $280 \mathrm{~nm}$ light emitting diode was frequency modulated in the range between 5 to $250 \mathrm{MHz}$. The emission was collected 
through a $320 \mathrm{~nm}$ long pass filter (Andover Inc.) and detected using a photomultiplier tube (PMT) (R928, Hamamatsu). The fluorescence decay data were analyzed using a combination of a single Gaussian distribution and a single discrete component according to equations 3.54 and 3.55 (Togashi et al., 2010).

$$
\begin{aligned}
& I(t)=a_{1} \int_{0}^{\infty} \rho(\tau)^{-t / \tau} d \tau+a_{2} \exp -\left(t / \tau_{2}\right) \\
& \rho(\tau)=\frac{1}{w_{1} \sqrt{2 \pi}} \exp \left[-\frac{1}{2}\left(\frac{\tau-\tau_{1}}{w_{1}}\right)^{2}\right]
\end{aligned}
$$

In these expressions, $a_{1}$ and $a_{2}$ represent the amplitude of each term in equation 3.54 and $\tau_{1}$ is the mean decay time of the Gaussian distribution with a width of distribution, $w_{l}$, whereas $\tau_{2}$ is the lifetime of the discrete single exponential term. The data analysis was performed using the Globals for Spectroscopy Software (Laboratory of Fluorescence Dynamics, University of California, Irvine, USA). The reference compound used in lifetime measurements was 2,5-diphenyloxazole (PPO) in ethanol ( $\tau=1.40 \mathrm{~ns})$ (Boens et al., 2007).

The average lifetimes $\langle\tau\rangle$ in were calculated using equations 3.56 and 3.57 .

$$
\begin{array}{r}
<\tau>=\frac{\alpha_{1} \tau_{1}^{2}+\alpha_{2} \tau_{2}^{2}}{\alpha_{1} \tau_{1}+\alpha_{2} \tau_{2}} \\
f_{i}=\frac{\int_{0}^{\infty} \alpha_{i}(\tau) \cdot \tau_{i} d \tau}{\int_{0}^{\infty} \Sigma_{i} \alpha_{i}(\tau) \cdot \tau_{i} d \tau}
\end{array}
$$


In these expressions, $\tau_{1}$ and $\tau_{2}$ are fluorescence lifetime components corresponding to the normalized pre-exponential decay $\alpha_{1}$ and $\alpha_{2}$ calculated from the exponential decay fraction $\left(f_{i}\right)$ using equation 3.57 , respectively.

\subsubsection{DREAM stability studies}

Guanidine hydrochloride $(\mathrm{GuHCl})$ induced protein unfolding was monitored by measuring the $\mathrm{CD}$ spectra as a function of increasing $\mathrm{GuHCl}$ concentration. Samples for CD measurements were prepared by solubilizing $40 \mu \mathrm{M}$ DREAM in $20 \mathrm{mM}$ Tris- $\mathrm{HCl}, 1$ mM DTT, $10 \mathrm{mMLDAO}, \mathrm{pH} 7.4$, and incubated in the presence of $\mathrm{GuHCl}$ for $30 \mathrm{~min}$ prior to CD measurements. The CD spectra were recorded using a Jasco J-810 CD-spectrometer at $16{ }^{\circ} \mathrm{C}$. DREAM unfolding traces were analyzed as a two-step process involving an intermediate state described by expression 3.58 .

$$
\mathrm{N} \leftrightarrow \mathrm{I} \leftrightarrow \mathrm{U}
$$

In this expression, $\mathrm{N}$ corresponds to the native state, I to the partially unfolded intermediate state, and $\mathrm{U}$ to the unfolded state.

The unfolding curves were fit using the following equation (Santoro and Bolen, 1988; Hung et al., 2003).

$$
Y(G u H C l)=\frac{Y_{N}+Y_{I} \exp \left[\frac{-\left(\Delta G_{I}^{\circ}-m_{I}[D]\right)}{R T}\right]+Y_{U} \exp \left[\frac{-\left(\Delta G_{I}^{\circ}-m_{I}[D]+\Delta G_{U}^{\circ}-m_{U}[D]\right)}{R T}\right]}{1+\exp \left[\frac{-\left(\Delta G_{I}^{\circ}-m_{I}[D]\right)}{R T}\right]+\exp \left[\frac{-\left(\Delta G_{I}^{\circ}-m_{I}[D]+\Delta G_{U}^{\circ}-m_{U}[D]\right)}{R T}\right]}
$$

In this equation, $\mathrm{Y}(\mathrm{GuHCl})$ is the $\mathrm{CD}$ signal at $220 \mathrm{~nm}, \Delta \mathrm{G}$ is the standard free energy for the unfolding, $\mathrm{m}$ is the dependence of the free energy on the denaturant concentration, $\mathrm{T}$ is the temperature $(\mathrm{T}=289 \mathrm{~K}),[\mathrm{D}]$ is the concentration of $\mathrm{GuHCl}$, and $\mathrm{R}$ is the ideal gas 
constant, $\mathrm{R}=8.314 \mathrm{~J} \mathrm{~K}^{-1} \mathrm{~mol}^{-1}$. The parameters $\mathrm{Y}_{\mathrm{N}}, \mathrm{Y}_{\mathrm{I}}$, and $\mathrm{Y}_{\mathrm{U}}$ represent the $\mathrm{CD}$ signal at $220 \mathrm{~nm} \mathrm{CD}$ signal for each molecular species at zero $\mathrm{GuHCl}$ concentration.

\subsubsection{Molecular dynamics simulations}

The molecular dynamics (MD) simulations in this chapter were performed in collaboration with Dr. Prem Chapagain in the Department of Physics, Florida International University. The time series MD trajectories were obtained from explicit solvent, all-atom simulations using the molecular dynamics simulation package NAMD (Phillips et al., 2005) with the CHARMM27 force field (Brooks et al., 1983). The initial NMR structure of the DREAM protein was obtained from the Protein Data Bank (PBS code: 2JUL (Lusin et al., 2008)). The protein was solvated using the VMD package (Humphrey et al., 1996) with a box cutoff set to $10 \AA$. This resulted in a simulation box of dimensions

$81.5 \times 68.6 \times 58.1 \AA$ A. The solvated system was electrically neutralized by adding $12 \mathrm{Na}^{+}$ions randomly in the bulk water using the VMD autoionize plugin. The particle mesh Ewald method (van Dijk et al., 2006) was used to treat long-range interactions with a $12 \AA$ nonbonding cutoff. Energy minimization (50000 steps) was performed using the conjugate gradient and line search algorithm. The system was then heated for 90 ps with a linear gradient of $20 \mathrm{~K} / 6 \mathrm{ps}$ from 20 to $300 \mathrm{~K}$. At $300 \mathrm{~K}$, the system was equilibrated for $910 \mathrm{ps}$ with a 2 fs integration time step in the NVT (constant number, volume, and temperature) ensemble. Langevin dynamics was used to maintain the temperature at $300 \mathrm{~K}$. The production run was 67 ns using NVT dynamics with 2 fs time steps. The same procedure was used to setup another system of a DREAM protein without calcium ions to simulate the Apo state of the protein. An additional $270 \mathrm{~ns}$ simulation was conducted for calcium 
bound DREAM using AMBER03 force fields with a $10 \AA$ PME cut off and 1.25 fs integration time, all other parameters being identical.

\subsection{Methods used in chapter 5}

\subsubsection{Fluorescence steady-state measurements}

Samples for fluorescence measurements were prepared by diluting DREAM-C in $20 \mathrm{mM}$ Tris- $\mathrm{HCl}, \mathrm{pH}$ 7.4, and $1 \mathrm{mM}$ DTT. Appropriate volume of $1 \mathrm{mM}$ EDTA, $5 \mathrm{mM}$ $\mathrm{Mg}^{2+}, 1 \mathrm{mM}$ EGTA, or $1 \mathrm{mM} \mathrm{Ca}^{2+}$, was added into $40 \mu \mathrm{M}$ DREAM-C samples to prepare Apo, $\mathrm{Mg}^{2+}, \mathrm{Ca}^{2+}$, or $\mathrm{Ca}^{2+} \mathrm{Mg}^{2+}$ bound protein.

Steady-state emission spectra of W169 and acrylamide quenching measurements were carried out similar to that performed with DREAM wild-type (sections 3.2.3).

Emission spectra of 1, 8-ANS and nile red in the presence of DREAM-C were measured using $370 \mathrm{~nm}$ and $470 \mathrm{~nm}$ excitation, respectively. The concentration of 1, 8ANS $\left(\varepsilon_{372 \mathrm{~nm}}=7,800 \mathrm{M}^{-1} \mathrm{~cm}^{-1}\right)$ (Diwu et al., 1997) and nile red $\left(\varepsilon_{552 \mathrm{~nm}}=19,600 \mathrm{M}^{-1} \mathrm{~cm}^{-1} \mathrm{in}\right.$ DMSO) (Castro et al., 2005) were determined spectrophotometrically. Equilibrium dissociation constants for 1,8-ANS or nile red were obtained by titrating concentrated protein into 1,8-ANS or nile red solution, while keeping the concentration of the fluorescence probe constant. Dissociation constants were recovered by nonlinear fit of the titration curves using a non-cooperative single binding site Hill equation as described in reference (Hawe et al., 2011).

\subsubsection{Fluorescence lifetime measurements}

Frequency-domain fluorescence lifetime and anisotropy decay measurements were performed using a ChronosFD spectrofluorometer (ISS, Champaign, IL). The frequency modulated output of a $280 \mathrm{~nm}$ laser diode was used as excitation light in Trp lifetime 
measurements and the emission was collected through a $320 \mathrm{~nm}$ long pass filter. 2,5diphenyloxazole (PPO) in ethanol $(\tau=1.40 \mathrm{~ns})$ was used as a lifetime reference (Boens et al., 2007). The lifetime of 1,8-ANS and nile red were determined using a frequency modulated $370 \mathrm{~nm}$ and $470 \mathrm{~nm}$ laser diode as an excitation source, respectively, and emission was collected using 400 - $600 \mathrm{~nm}$ and 550 - $700 \mathrm{~nm}$ band pass filters, respectively. The fluorescence lifetime of 1,4-bis(5-phenyloxazol-2-yl)benzene (POPOP) in ethanol ( $\tau$ $=1.35 \mathrm{~ns})$ (Boens et al., 2007) and rhodamine B in water $(\tau=1.7 \mathrm{~ns})($ Boens et al, 2007) were employed as lifetime references for 1,8-ANS and nile red, respectively. Multiple lifetime data from at least three different protein preparations were simultaneously fit using the global analysis approach with the lifetime parameters set as linked variables (Global WE software, Laboratory of Fluorescence Dynamics).

The time-resolved anisotropy measurements of 1, 8-ANS:DREAM-C complexes were performed as described previously(Gonzalez and Miksovska, 2014). A double associative decay model was used to determine the rotational correlation times. The $\chi^{2}$ parameter and residuals were used as criterion for goodness of fit for all the models used.

\subsubsection{DREAM-C stability measurements}

Guanidine hydrochloride $(\mathrm{GuHCl})$ stock solution was prepared by dissolving $\mathrm{GuHCl}$ in $20 \mathrm{mM}$ Tris-HCl, pH 7.4, and $1 \mathrm{mM}$ DTT buffer to obtain a final concentration of $\mathrm{GuHCl}$ of $8.0 \mathrm{M}$. Thirty eight samples for each form of Apo, $\mathrm{Mg}^{2+}, \mathrm{Ca}^{2+}, \mathrm{or} \mathrm{Ca}^{2+} / \mathrm{Mg}^{2+}$ bound DREAM-C at a constant protein concentration $(40 \mu \mathrm{M})$ and various $\mathrm{GuHCl}$ concentrations $(0.2-7.4 \mathrm{M})$ were prepared for unfolding measurements. To ensure that an unfolding equilibrium was reached, samples were incubated at $20{ }^{\circ} \mathrm{C}$ for 30 min prior to each measurement. 
Far UV-CD spectra were recorded on JASCO J-810 CD spectropolarimeter (JASCO Inc. Easton, MD) from 260 to $200 \mathrm{~nm}$ using $0.1 \mathrm{~cm}$ path length quartz cell. Protein unfolding curves were obtained by monitoring changes in ellipticity at $220 \mathrm{~nm}$ as a function of $\mathrm{GuHCl}$ concentrations. Unfolding curves were analyzed using two-state unfolding model $N \leftrightarrow U$ (Santoro and Bolen, 1988; Hung et al., 2001).

$$
Y_{o b s}=\left\{Y_{N}+Y_{U} \exp \left(\frac{-\left(\Delta G_{U}-m_{U}[D]\right)}{R T}\right)\right\} /\left\{1+\exp \left(\frac{-\left(\Delta G_{U}-m_{U}[D]\right)}{R T}\right)\right\}
$$

In this expression, the observed CD signal $\left(Y_{o b s}\right)$ is expressed in terms of the signal of the native state $\left(Y_{N}\right)$ and unfolded state $\left(Y_{U}\right) . \Delta G_{U}$ represents the unfolding free energy change, $\mathrm{m}_{\mathrm{U}}$ describes the free energy dependences on the denaturant concentration $([D]), \mathrm{R}$ is the universal gas constant $\left(1.987 \mathrm{cal} \mathrm{mol}^{-1} \mathrm{~K}^{-1}\right)$, and $\mathrm{T}$ is the temperature.

The fraction of the native state was determined from the unfolding equilibrium constant using equations 3.61 and 3.62 .

$$
\begin{aligned}
& K_{U}=\exp \left(\frac{-\Delta G_{U}}{R T}\right) \\
& f_{N}=\frac{1}{\left(1+K_{U}\right)}
\end{aligned}
$$

\subsubsection{Isothermal Titration Calorimetry (ITC) measurements}

Thermodynamic parameters for $\mathrm{Ca}^{2+}$ and $\mathrm{Mg}^{2+}$ binding to DREAM-C were determined using a VP-ITC titration calorimeter (Microcal Inc. Northampton, MA). ITC buffer (20 mM Tris-HCl, pH 7.6, 0.5 mM DTT, and $150 \mathrm{mM} \mathrm{NaCl}$ ) was prepared using decalcified water and filtered through Chelex-100 resin (Bio-Rad). The protein stock solution was dialyzed against ITC buffer overnight. To minimize artifacts from 
mismatched buffers, $1.2 \mathrm{mM} \mathrm{CaCl}_{2}$ and $5 \mathrm{mM} \mathrm{Mg} \mathrm{Cl}_{2}$ solutions were prepared by diluting $0.1 \mathrm{M} \mathrm{CaCl}_{2}$ standard solution and $1.0 \mathrm{M} \mathrm{MgCl}_{2}$ solution in the final ITC buffer, respectively. All buffer solutions were stored in plastic bottles previously boiled in decalcified water. The cell sample and injection syringe were extensively cleaned with decalcified water and then with ITC buffer. The reaction cell was loaded with $70 \mu \mathrm{M}$ DREAM-C solution $(1.40 \mathrm{~mL})$ and the concentration of $\mathrm{Ca}^{2+}$ or $\mathrm{Mg}^{2+}$ in syringe $(297 \mu \mathrm{L})$ was $1.2 \mathrm{mM}$ or $5.0 \mathrm{mM}$, respectively. For $\mathrm{Ca}^{2+}$ binding to DREAM-C in presence of $\mathrm{Mg}^{2+}$, $1.2 \mathrm{mM}$ solution of $\mathrm{Ca}^{2+}$ was titrated into $70 \mu \mathrm{M}$ DREAM-C that was pre-saturated with 5 $\mathrm{mM} \mathrm{Mg}{ }^{2+}$. Twenty eight aliquots $(10 \mu \mathrm{L}$ each $)$ of $\mathrm{Ca}^{2+}$ or $\mathrm{Mg}^{2+}$ solutions were titrated into protein solution with 2 min intervals between injections. Parallel experiments were carried out for titration of $\mathrm{Ca}^{2+}$ or $\mathrm{Mg}^{2+}$ solution into the dialysate buffer as a control for heats of dilutions of ligand. The temperature was kept at $25^{\circ} \mathrm{C}$ and the stirring speed was kept at 307 rpm. ITC data were analyzed using Origin 7 ITC data analysis software (OriginLab Corp. Northampton, MA). The data obtained for $\mathrm{Ca}^{2+}$ binding to DREAM-C and $\mathrm{Mg}^{2+}$ DREAM-C were analyzed using a two binding sites model as described previously (Jelesarov and Bosshard, 1999; Martinez et al., 2013).

\subsection{Methods used in chapter 6}

\subsubsection{Sample preparation}

Fluorescence samples were prepared from stock protein diluted in a buffer containing $20 \mathrm{mM}$ Tris-HCl, $\mathrm{pH}$ 7.4, and $1 \mathrm{mM}$ DTT. Presenilin 1 C-terminal fragment (PS1-CTF) peptides were freshly solubilized in the same buffer and stored at $4{ }^{\circ} \mathrm{C}$. The peptide concentration was determined spectrophotometrically using extinction coefficient 
of fluorescein isothyocyanate (FITC), $\varepsilon_{493 \mathrm{~nm}}=75,000 \mathrm{M}^{-1} \mathrm{~cm}^{-1}$. Absorption spectra were measured using a single beam spectrophotometer (Cary-50, Varian).

\subsubsection{Fluorescence steady-state measurements}

Fluorescence steady-state anisotropy measurements were performed on a ChronosFD spectrofluorometer (ISS Inc., Champaign, IL). The samples were excited at $470 \mathrm{~nm}$ and the emission was collected using 500-700 $\mathrm{nm}$ band pass filters (Andover corp. Salem, NH). Titrations of HL $\beta$ and HL9 into DREAM were conducted in a $2 \mathrm{~mm} \times 10$ mm quartz cuvette with excitation along the short path-length. Equilibrium dissociation constants $\left(\mathrm{K}_{\mathrm{d}}\right)$ were determined by a non-linear fit of the change in fluorescence anisotropy using equation 1 (Leon et al., 2009).

$$
\Delta A=\frac{F}{2}\left[K_{d}+n P_{t}+L_{t}-\sqrt{\left(K_{d}+n P_{t}+L_{t}\right)^{2}-4 n P_{t} L_{t}}\right]
$$

where $\Delta A$ is change in fluorescence anisotropy, $\mathrm{K}_{\mathrm{d}}$ is dissociation constant, $n$ is the number of binding sites, $P_{t}$ is the total DREAM protein concentration, $L_{t}$ is total PS1 peptide concentration, and $F$ is the fluorescence anisotropy scaling factor.

For PS1 peptide displacement studies, the NS5806 drug compound (Calloe et al., 2009) was titrated into $\mathrm{Ca}^{2+}$ DREAM and PS1-HL9 complex. The dissociation constant of NS5806 for $\mathrm{Ca}^{2+}$ DREAM was recovered using a single binding site displacement equation 3.64 (Epps et al., 1995; Chuang et al., 2008; Gonzalez et al., 2014).

$$
\frac{\Delta \text { Anisotropy }_{\text {measured }}}{\Delta \text { Anisotropy }_{\max }}=\alpha+\frac{\beta\left[L_{t}\right]}{\left(K_{i}+\left[L_{t}\right]\right)\left(1+\frac{I_{t}}{K_{d}}\right)}
$$


In this expression, $\Delta$ Anisotropy is change in fluorescence steady-state anisotropy. Parameters $\alpha$ and $\beta$ are proportionality constants, $K_{i}$ is the dissociation constant of PS1HL9 and $\mathrm{Ca}^{2+}$ DREAM (measured separately, mentioned in previous sections), $\mathrm{I}_{\mathrm{t}}$ is the total concentration of NS5806 and $\mathrm{K}_{\mathrm{d}}$ is its dissociation constant.

\subsubsection{Fluorescence time-resolved anisotropy measurements}

Anisotropy decay was measured using a $470 \mathrm{~nm}$ laser diode, and the emission was collected through 500-700 nm band pass filters (Andover corp. Salem, NH). Anisotropy data were analyzed using a double-exponential decay model (VINCI software, ISS Inc., Champaign, IL) described by equation 3.44 or 3.45 in section 3.2.5.3, assuming a spherical shape of DREAM-peptide complex (Jameson, 2014).

\subsubsection{Fluorescence stop-flow kinetics}

The kinetics of DREAM binding to HL9 were measured with a ChronosFD spectrofluorometer coupled to a SFA-20 rapid stopped-flow apparatus (TgK Scientific, Bradford-on-Avon, UK). The cell was illuminated with $295 \mathrm{~nm}$ excitation and fluorescence emission at $520 \mathrm{~nm}$ due to fluorescence resonance energy transfer (FRET) from the single tryptophan residue (W169) in DREAM (donor) to FITC (acceptor) labeled on HL9 was monitored. In control experiments, the buffer $\left(20 \mathrm{mM}\right.$ Tris- $\mathrm{HCl}$, $\mathrm{pH} 7.4,1 \mathrm{mM} \mathrm{CaCl}_{2}$, and $1 \mathrm{mM}$ DTT) was used instead of $\mathrm{Ca}^{2+}$ DREAM protein. Kinetics data were analyzed using single and double exponential decay models (Origin 8 software, OriginLab Corp. Northampton, MA).

\subsubsection{Computational protein-protein docking study}

The NMR structures of $\mathrm{Ca}^{2+}$ bound DREAM (PDB: 2JUL, molecule 1) and PS1CTF (PDB: 2KR6, molecule 1) were used as receptor and ligand inputs, respectively. The 
inter-protein complex of DREAM and PS1-CTF was performed on the ClusPro 2.0 proteinprotein docking server (Kozakov et al., 2006; Kozakov et al. 2013; Comeau et al, 2004; Comeau et al., 2004). In the first step, ClusPro 2.0 program uses PIPER, a fast Fourier transform based docking program, to calculate a pairwise interaction potential as part of its scoring as shown in equation 3.65 .

$$
E=E_{\text {attr }}+w_{1} E_{\text {rep }}+w_{2} E_{\text {elec }}+w_{3} E_{\text {pair }}
$$

where $E_{a t t r}$ and $E_{r e p}$ are the attractive and repulsive forces contributing to the van der Waals interaction energy $\left(E_{v d w}\right)$, whereas $E_{\text {elec }}$ and $E_{\text {pair }}$ are electrostatic and desolvation energies, respectively. The coefficients $w_{1}, w_{2}$, and $w_{3}$ are the weights of the corresponding energetic terms (Kozakov et al., 2006; Kozakov et al., 2013). After performing PIPER docking, the best 1,000 energy structures were clustered for ranging by using pairwise root mean square deviation (RMSD) as the distance measured (Comeau et al., 2004). The biophysical meaning of clustering is to isolate energy basins of highly populated energy areas (Kozakov et al., 2013). Finally, the output clusters are displayed in scoring schemes namely balanced, electrostatic-favored, hydrophobic favored, and van der Waals and electrostatics complexes. There are several available protein-protein docking programs such as ZDOCK (Pierce et al., 2014), RosettaDock (Lyskov et al., 2008), and HADDOCK (de Vries et al., 2010). However, ClusPro 2.0 was chosen for performing $\mathrm{Ca}^{2+}$ DREAM and PS1 docking as it uses the PIPER algorithm to rapidly evaluate protein-protein interactions. In addition, since experimental results showed that interactions between $\mathrm{Ca}^{2+}$ DREAM and PS1-HL9 are driven by not only electrostatic interactions but also hydrophobic interactions, various post-docking processing methods available in ClusPro 2.0 score the resultant complexes of $\mathrm{Ca}^{2+}$ DREAM-PS1-CTF in a manner that can be comparable with experimental data. The 
predicted $\mathrm{Ca}^{2+}$ DREAM-PS1-CTF structure was analyzed using the YASARA Structure program version 13.1.25 (Krieger et al., 2002) and the academic version of PyMOL 1.7.4 software (Schrodinger, 2015).

\subsection{Methods used in chapter 7}

\subsubsection{Tryptophan steady-state emission measurements}

The fluorescence emission of tryptophan (W169) in DREAM-EF hand mutants D150N, E186Q, and E234Q was measured as described for DREAM wild-type in chapter 4 (section 3.3.1).

\subsubsection{Tryptophan fluorescence lifetime measurements}

The frequency-domain fluorescence lifetime measurements of W169 in DREAMEF hand mutants D150N, E186Q, and E234Q and fluorescence data analysis were carried out as described for DREAM wild-type in chapter 4 (section 3.3.3).

\subsubsection{Fluorescence anisotropy decay measurements}

Oligomeric states of the DREAM-DNA complex were resolved by fluorescence anisotropy decay measurements. Ethidium bromide was (EtBr) used as a fluorescence probe since its fluorescence emission and lifetime significantly increase in the presence of double stranded DNA (Garbett et al., 2004; Lakowicz, 2006). Time-resolved anisotropy was measured using a $470 \mathrm{~nm}$ laser diode and the emission was collected through $500-700$ nm band pass filters (Andover corp. Salem, NH). Anisotropy decay data were analyzed using equations 3.44 and 3.45 as described previously in section 3.2.5.3 for a mixed system of free DNA and the DNA bound DREAM complex, assuming a spherical shape for both systems. (VINCI software, ISS Inc., Champaign, IL) (Jameson, 2014). 


\subsubsection{Circular dichroism measurements of DREAM mutants}

Circular dichroism (CD) measurements of DREAM EF-hand mutants were performed with a Jasco J-815 CD spectropolarimeter. All CD spectra of DREAM mutants were measured at $40 \mu \mathrm{M}$ protein concentration through a $1 \mathrm{~mm}$ path in $20 \mathrm{mM}$ Tris- $\mathrm{HCl}$ buffer, pH 7.4, 1 mM DTT, and 10 mM LDAO. All measurements were conducted at 16 ${ }^{\circ} \mathrm{C}$, and each $\mathrm{CD}$ spectrum represents an average of 5 scans.

\subsubsection{Thermodynamics of DREAM and DNA complex}

Isothermal titration calorimetry (ITC) was employed to measure the thermodynamic interactions between DREAM/DREAM mutants and DNA. These measurements were performed using a VP-ITC MicroCalorimeter (MicroCal LLC, Northampton MA). The ITC buffer (20 mM Tris-HCl, $0.5 \mathrm{mM}$ DTT, and $10 \mathrm{mM}$ LDAO, $\mathrm{pH}$ 7.4) was prepared using decalcified water filtered through a Chelex-100 resin (BioRad) column. Purified DREAM and DREAM EF-hand mutants were dialyzed overnight against the ITC buffer, and the DNA sample was prepared in the ITC buffer (20 mM Tris$\mathrm{HCl}, 0.5 \mathrm{mM}$ DTT, and $10 \mathrm{mM}$ LDAO, $\mathrm{pH}$ 7.4) in order to minimize artifacts due to mismatched buffers. The cell sample and injection syringe were extensively cleaned with decalcified water and then with the ITC buffer. The reaction cell was filled with $15 \mu \mathrm{M}$ dsDNA solution $(1.40 \mathrm{~mL})$, and the injection syringe was loaded with $500-550 \mu \mathrm{M}$ DREAM protein solution $(297 \mu \mathrm{L})$. For measurements of $\mathrm{Mg}^{2+}$ DREAM and $\mathrm{Ca}^{2+} \mathrm{DREAM}$ binding to DNA, both DREAM and DNA samples were prepared in the presence of 100 $\mu \mathrm{M} \mathrm{Mg}^{2+}$ and $1 \mathrm{mM} \mathrm{Ca}^{2+}$, respectively. Twenty nine aliquots (with injection volume set to $4 \mu \mathrm{L}$ for the first injection, and $10 \mu \mathrm{L}$ for the following injections) of DREAM were titrated into DNA solution with 2 min intervals between injections. The temperature was kept at 
$25{ }^{\circ} \mathrm{C}$ and the stirring speed was set at $307 \mathrm{rpm}$. Parallel experiments were carried out for titration of DREAM into the dialysate buffer as a control for the heats of dilution. ITC data were analyzed using the Origin 7 software package provided by the manufacturer (OriginLab Corp. Northampton, MA). The first titration point from each ITC experiment was deleted when fitting to binding models due to possible diffusive mixing of protein near the tip of the syringe. The data obtained for DREAM and DREAM EF-hand mutants binding to DNA were analyzed using a two binding sites model, except for the analysis of $\mathrm{Ca}^{2+}$ DREAM binding to DNA which was fitted using a sequential binding sites model (Jelesarov and Bosshard, 1999; Martinez et al., 2013).

\subsubsection{Molecular dynamics simulations and modeling of DREAM and DREAM mutants}

Since 3D structures of both Apo and $\mathrm{Mg}^{2+}$ DREAM are not available, the MD simulation structure of ApoDREAM was constructed from the NMR structure of $\mathrm{Ca}^{2+}$ DREAM (PDB: 2JUL, molecule \#1 (Lusin et al., 2008)) by deleting the two $\mathrm{Ca}^{2+}$ atoms. In addition, the structure of $\mathrm{Mg}^{2+} \mathrm{DREAM}$ was also constructed from the NMR structure of $\mathrm{Ca}^{2+}$ DREAM (PDB: 2JUL, molecule \#1 (Lusin et al., 2008)) with the $\mathrm{Mg}^{2+}$ binding coordination at EF-hand 2 (residues 139 - 150) being replaced by the $\mathrm{Mg}^{2+}$ binding coordination (residues 116 - 127) from the NMR structure of $\mathrm{Mg}^{2+}$ bound calcium-andintegrin-binding protein $1\left(\mathrm{Mg}^{2+} \mathrm{CIB} 1\right.$, PDB entry: 2L4I) (Huang et al., 2011). The other mismatched residues within the $\mathrm{Mg}^{2+}$ binding loop of $\mathrm{Mg}^{2+} \mathrm{CIB} 1$ were also replaced with the corresponding residues from the $\mathrm{Mg}^{2+}$ binding loop of DREAM. The coordinative binding of $\mathrm{Mg}^{2+}$ in the structure of $\mathrm{Mg}^{2+} \mathrm{CIB} 1$ was chosen because the critical residues at positions $1,3,5$, and 12 in the EF-hand $\mathrm{Mg}^{2+}$ binding loop of CIB1 (DxDxDxxxxxxD; 
is any amino acid) are close in homology to that of DREAM EF-hand 2 (DxDxNxxxxxxD; $\mathrm{x}$ is any amino acid). In addition, I observed that the geometry of the $\mathrm{Mg}^{2+}$ binding loop in CIB1 is very close to that of DREAM protein. Moreover, the structures of DREAM EFhand mutants $(\mathrm{D} 150 \rightarrow \mathrm{N}, \mathrm{E} 186 \rightarrow \mathrm{Q}$, and E234 $\rightarrow$ Q) were constructed using the plugin mutagenesis feature in the CHARMM-GUI web server (Jo et al., 2008) using the NMR structure of $\mathrm{Ca}^{2+}$ DREAM (PDB: 2JUL, molecule \#1 (Lusin et al., 2008)) as the initial structure. The CHARMM-GUI web server was utilized to prepare the initial coordination files for MD simulations (Jo et al., 2008). The DREAM models were solvated using TIP3P waters, in a rectangular box with $10 \AA$ distance between edges of a biomolecule and those of the solvation box. The system was neutralized with $0.15 \mathrm{M} \mathrm{NaCl}\left(59 \mathrm{Na}^{+}\right.$ions and 39 $\mathrm{Cl}^{-}$ions), and the initial configuration of ions was determined through short Monte Carlo (MC) simulation (2000 steps) using a primitive model (Jo et al., 2008). The simulations were performed under periodic boundary conditions (PBC) and with the Particle-Mesh Ewald (PME) method (van Dijk et al., 2006) with a 12 Å nonbonding cutoff (Jo et al., 2008). Energy minimization (50000 steps) was performed using the SHAKE algorithm, while the system was set at a temperature of $303.15 \mathrm{~K}$ and a 2 fs time step in NVT (constant volume and temperature) (Jo et al., 2008). The production run was $100 \mathrm{~ns}$ using NPT (constant pressure and temperature) dynamics with 2 fs time steps (Jo et al., 2008). All the bond lengths involving hydrogen atoms were fixed for both energy minimization and production runs. Simulations were carried out using the NAMD (Phillips et al., 2005) simulation software and the CHARMM27 force field with CMAP corrections (Mackerell, 2004; Bjelkmar et al., 2010). All simulations of ApoDREAM, ApoDREAM-D150N, $\mathrm{Mg}^{2+}$ DREAM, $\mathrm{Ca}^{2+}$ DREAM, $\mathrm{Ca}^{2+}$ DREAM-E186Q, and $\mathrm{Ca}^{2+}$ DREAM-E234Q were 
performed on an Intel i7-4770 and GTX-TITAN black super-clocked (1072 MHz) system using NAMD 2.11 (NVIDIA CUDA acceleration) (Phillips et al., 2005). Trajectory files were saved every $20 \mathrm{ps}$. The frames of all simulations of Apo and $\mathrm{Ca}^{2+}$ DREAM were then compared with that obtained for Apo and $\mathrm{Ca}^{2+}$ DREAM from our previous studies in chapter 4 (Pham et al., 2015) to verify accuracy of the simulations.

\subsubsection{Analysis of MD simulations}

Calculations of root mean square deviations (RMSD) and root mean squared fluctuations (RMSF) of DREAM protein were performed using VMD 1.9.2 software (Humphrey et al., 1996). The RMSD was calculated for the alpha carbons $\left(\mathrm{C}_{\alpha}\right)$ of the whole protein (residues 76 to 256) using the equilibrated structure of the corresponding DREAM as the frame of reference. The RMSF were calculated using an average trajectory simulation of all alpha carbons of DREAM protein between 20 and $100 \mathrm{~ns}$ simulation time (to skip the relaxation period at the beginning of the simulation time $(0-20 \mathrm{~ns})$ ). Conformational dynamics of DREAM and DREAM mutants were analyzed using dynamic network analysis of 100 ns simulation trajectory. The dynamical network analysis was performed using the VMD plugin Network Setup feature (Sethi et al., 2009). In dynamic network analysis, each node represents the alpha carbon of an amino acid, and the edges connecting non-neighboring alpha carbons represent atoms which interacted over $75 \%$ of the time within $4.5 \AA$. The thickness of the edges represents the degree of correlation between the nodes. These networks were then classified into communities of dynamically coupled residues by using the Girvan-Newman algorithm (Girvan and Newman, 2002). Within a given community, the amino acids are more correlated to each other than to the amino acids in the neighboring communities. The connections between communities 
represent dynamic hubs, which reflects the dynamic fluctuations from the most propagated community to the rest.

\subsubsection{Computational docking of DREAM and DNA interaction}

The initial structure of ApoDREAM protein was obtained from 100 ns MD simulation of ApoDREAM. The standard B-DNA conformation of the DRE sequence (5'GAAGCCGGAGTCAAGGAGGCCCCTG-3') of prodynorphin gene was built using a 3D-DART (3DNA-Driven DNA analysis and rebuilding tools) modelling server (van Dijk and Bonvin, 2009). There are several available programs including 3D-DART that convert the sequence of DNA into a 3D structure using regular nucleotide building blocks (Dixit and Beveridge, 2006; Lu and Olson, 2003; van Dijk and Bonvin, 2009). The high accuracy of these programs was discussed elsewhere (van Dijk and Bonvin, 2009). In this study, 3DDART program was chosen because the server not only returns the $3 \mathrm{D}$-structural models of the double stranded DNA, but it also allows us to generate the DNA restraint file for DREAM and DNA docking input used in the HADDOCK (High Ambiguity-Driven DOCKing) program (van Dijk and Bonvin 2009; de Vries et al., 2010; Wassenaar et al., 2012). The ambiguous interaction restraints (AIR), including the active residues and nucleotides in DREAM and DNA located at the predicted interaction interface, were determined based on experimental results previously published (Carrion et al. 1999; Ledo et al., 2000; Lusin et al., 2008). The HADDOCK 2.2 web server was used to build models for ApoDREAM-DNA complexes from the unbound structures of ApoDREAM and double stranded DNA (Wassenaar et al., 2012; de Vries et al., 2010). The HADDOCK docking program was run with default settings. Residues K87, K90, K91, R98, K101, R160, and K166 in the ApoDREAM protein and nucleotides G10, T11, C12, A13, T38, 
G39, A40, and C41 in the double stranded DNA were designated as active restraints to drive the docking. Passive residues and nucleotides, defined as the solvent accessible neighbors of active residues, were automatically determined by the HADDOCK program (Wassenaar et al., 2012; de Vries et al., 2010). Initially, 1000 complexes of the ApoDREAM and DNA were generated by performing rigid-body docking and the lowest rigid body score (R.B. score), calculated using equation 3.66, was selected (van Dijk and Bonvin, 2009).

$$
\text { R.B.score }=1.0 * \text { Elec }+1.0 * v d W-0.05 * B S A+1.0 * \text { Dsolv }+1.0 \text { AIR }
$$

In this expression, the parameters Elec, $v d W, B S A, D s o l v$, and AIR are intermolecular electrostatic, van der Waals, buried surface are, desolvation, and AIR energies, respectively. Following rigid-body docking, 200 complexes with best rigid-body scores were selected for further refinement using the semi-flexible docking, in which side chains and the backbone of the protein at the predicted interface and the entire DNA were allowed to move (van Dijk and Bonvin 2009). Finally, water refinement of the ApoDREAM-DNA complex was performed with $100 \mathrm{MD}$ heating steps at 100, 200, and $300 \mathrm{~K}$ followed by sampling steps at $300 \mathrm{~K}$ and $500 \mathrm{MD}$ cooling steps at 300,200 , and $100 \mathrm{~K}$ in a water box (van Dijk and Bonvin, 2009). The final HADDOCK score (H.D. score) was recalculated using equation 3.67 (van Dijk and Bonvin, 2009).

$$
\text { H.D.score }=1.0 * E l c+1.0 * v d W+1.0 * \text { Dsolv }+1.0 * A I R
$$

The parameters Elc, $v d W, D s o l v$, and AIR were defined previously in equation 3.66. 


\subsection{Materials and methods used in chapter 8}

\subsubsection{Materials}

Chemicals such as urea, Tris buffer and proteins including horse heart myoglobin (Mb) and microperoxidase 11 (MP-11) were purchased from Sigma-Aldrich Chemical Co (St. Louis, MO). Bis (2-ethylhexyl) sulfosuccinate sodium salt (AOT) and sodium dithionite were purchased from Fluka Chemical Corp (Milwaukee, WI). ACS grade heptane $\left(\mathrm{C}_{7} \mathrm{H}_{16}\right)$ was purchased from Fisher Scientific (New Jersey 07410). Argon (Ar) and carbon monoxide $(\mathrm{CO})$ gases are in pressure bottles and purchased at purities at $99.97 \%$.

\subsubsection{Sample preparation}

A solution of $0.1 \mathrm{M}$ AOT reverse micelles was prepared by adding AOT to the mixture of heptane and varying amounts of water. The AOT mixture was stirred until the solution became transparent. The AOT sample was placed into a 1 x $1 \mathrm{~cm}$ quartz cuvette, sealed with a rubber septum cap and purged with Ar gas for $20 \mathrm{~min}$. Next, the desired volume of a stock solution of horse heart $\mathrm{Fe}^{2+} \mathrm{Mb}$ or $\mathrm{Fe}^{2+} \mathrm{MP}-11$ in $100 \mathrm{mM}$ Tris buffer, $\mathrm{pH}$ 7.0, was anaerobically injected into the AOT reverse micelle solution to achieve a final value of $\mathrm{w}_{\mathrm{o}}=40 \mathrm{in}$ a total solution volume of $3 \mathrm{~mL}$. The sample was then flushed with $\mathrm{CO}$ for at least $5 \mathrm{~min}$ and allowed to equilibrate for an additional $20 \mathrm{~min}$. Complete formation of $\mathrm{CO}-\mathrm{Mb}$ or CO-MP-11 was optically determined.

\subsubsection{Absorption measurements}

The UV/Vis absorption spectra were measured using a spectrophotometer (Varian/Carry 400, Varian Instruments, Walnut Creek, CA) from 350 to $700 \mathrm{~nm}$ using 1 $\mathrm{nm}$ steps, $0.5 \mathrm{~s} / \mathrm{step}$ and an auto adjusted baseline. The UV/Vis spectra provide 
verifications for the formation of the deoxy form (ferrous heme iron) and $\mathrm{CO}$ ligated formation by visual inspection absorbance at the Soret band.

\subsubsection{Transient absorption}

The ligand (CO) dynamic rebinding kinetics was studied by employing a homebuilt transient absorption instrument described in detail in (Miksovska et al., 2006, Larsen et al., 2011). Briefly, the photodissociation was trigged by a $532 \mathrm{~nm}, 7 \mathrm{~ns}$ width, and $1 \mathrm{~Hz}$ repetition pulse initiated by a double Nd:YAG laser pulse (Minilite II, Continuum). The probe beam generated from the 150-W Xe arc lamp (Thermo-Oriel) was passed through a monochromator, and guided through the sample housed in a $1.0 \mathrm{~cm}$ variable temperature controlled cell holder (Quantum Northwest). The emerging light was focused on the slit of the second monochromator (Micro HR, Jobin Yvon) and detected by a photomultiplier tube (PMT) connected to a high-gain amplifier. The photocurrent was converted into voltage and digitized by digital oscilloscope (Tektronix). Data were analyzed using OriginPro 8.0 software (OriginLab Corpration).

\subsubsection{Photoacoustic calorimetry}

The experimental setup has been described previously (Larsen et al. 2011). Briefly, a 7 ns pulse from a frequency doubled Nd:YAG laser (532 nm, Continuum Minilite II $\mathrm{Nd}$ :YAG laser) is passed through a pin-hole and directed onto the face of a temperature regulated cell holder (Quantum Northwest, temperature controled to within $0.01^{\circ} \mathrm{C}$ ) adapted for a $2 \mathrm{MHz}$ PZT piezoelectric transducer which is coupled to the face of the quartz sample cell via a thin layer of vacuum grease. The signal is amplified using a Panametrics model 5978 ultrasonic preamplifier and digitized by a Picoscope 3200 Oscilloscope running on a desktop PC. Deconvolution of the acoustic waves is performed with software 
developed by Dr. Randy Larsen in the department of Chemistry, University of South Florida (LarsenWare2009V1). Acoustic simulation software is also available. The LarsenWare2009V1 can deconvolute both single exponential decay models as well as stretched exponential decays. 


\section{CALCIUM AND MAGNESIUM MODULATE CONFORMATIONAL}

DYNAMICS AND STABILITY OF DOWNSTREAM REGULATORY ELEMENT ANTAGONIST MODULATOR

(The work described in this chapter was published in K., Pham, G., Dhulipala, W. G., Gonzalez, B. S., Gerstman, C., Regmi, P.P., Chapagain, J., Miksovska (2015), Protein Sci.,24(5):741-751)

\subsection{Background and significance}

The EF-hand motif $\mathrm{Ca}^{2+}$ binding proteins are involved in the regulation of numerous intracellular processes ranging from gene expression, cell division, cell growth, as well as apoptosis. All members of this family carry at least one pair of EF-hand calcium binding sites that bind calcium with equilibrium affinity constants ranging from $10^{4} \mathrm{M}$ in the case of S100 proteins, to $10^{8} \mathrm{M}$ as found for parvalbumin (Gifford et al., 2007). The protein affinity for $\mathrm{Ca}^{2+}$ is fine-tuned by the amino acid sequence of the EF-hand binding site, overall structure of the $\mathrm{Ca}^{2+}$ sensor, and interactions with other intracellular partners. Despite relatively high sequence similarities, EF-hand proteins exhibit large structural diversity in the Apo and $\mathrm{Ca}^{2+}$ bound forms that is essential for specific interactions with intracellular partners and functional diversity (Bhattacharya et al., 2004).

Depending on the amino acid sequence, structural and functional properties, and the organization of the $\mathrm{N}$ - and C- terminal domains, the intracellular EF-hand proteins can be divided into three groups. The members of the first group that includes calmodulin and troponin $\mathrm{C}$ have two independent domains connected by a flexible $\alpha$-helical linker. Calcium association leads to a substantial reorganization of the $\mathrm{C}$ - and $\mathrm{N}$-terminal domains and exposure of hydrophobic patches on the protein surface. In calmodulin, the highly 
dynamic protein structure enables specific interactions with a large number of intracellular targets (Bhattacharya et al., 2004). The second group of EF-hand proteins includes dimeric S100 proteins. Unlike the calmodulin family, structural changes linked to $\mathrm{Ca}^{2+}$ binding to EF-hands in S100 proteins are relatively modest and result in exposure of a wider and flatter hydrophobic crevice on the protein surface that facilitates binding to intracellular partners (Chazin, 2011).

The third group of EF-hand proteins consists of neuronal calcium sensors (NCS) that are predominantly expressed in neuronal tissue where they regulate numerous intracellular processes including vision transduction, potassium voltage channel kinetics, and DNA expression (Burgoyne and Weiss, 2001; Burgoyne, 2007; Ames and Lim, 2012). All members of NCS family carry four EF-hand binding sites, although only two or three EF-hands are active and bind bivalent ions with a high affinity (Ames and Lim, 2012). Some members of the NCS family undergo post-translational modifications such as fatty acid esterification at the $\mathrm{N}$-terminus. The presence of the fatty acid modulates the range of $\mathrm{Ca}^{2+}$ triggered conformational switching and facilitates protein association to the membrane (Haynes and Burgoyne, 2008). The C- and N-terminal domains in NCS are each organized in a globular arrangement and are connected by a short U-shaped linker with a solvent-exposed hydrophobic groove stretching between the $\mathrm{C}$ - and $\mathrm{N}$-terminal domains (Ames and Lim, 2012).

The DREAM protein, also known as KChIP3 or calsenilin, belongs to the subfamily of NCS potassium channel interacting proteins (KChIP), which includes three additional members (KChIP1, KChIP2, and KChIP4) (An et al., 2000). All members share a conserved region of 180 residues along with highly variable $\mathrm{N}$-termini of 35 to 100 residues 
(Schwenk et al., 2008). In DREAM, EF-hand 1 has no function due to the conserved residues $\mathrm{C} 104$ and $\mathrm{P} 105$ on the first $\mathrm{Ca}^{2+}$ binding loop which prevent this site from binding to $\mathrm{Ca}^{2+}$ and/or $\mathrm{Mg}^{2+}$. On the other hand, the canonical EF-hand 3 and EF-hand 4 functionally bind $\mathrm{Ca}^{2+}$ with a high affinity $(\mathrm{Kd} \sim 1-10 \mu \mathrm{M})$ and EF-hand 2 structurally binds $\mathrm{Mg}^{2+}(\mathrm{Kd} \sim 14 \mu \mathrm{M})$ (Osawa et al., 2005). KChIP1, DREAM, and KChIP4 are predominantly expressed in the brain where they regulate gating properties of Kv4.3 channels and the surface expression of the Kv4.3 complex (Jerng et al., 2004; Zhou et al., 2004). Unlike other members of the KChIP subfamily, DREAM interacts with numerous intracellular partners including calmodulin (Ramachandran et al., 2012), DNA (Carrion et al., 1999), presenilin (Buxbaum et al., 1998; Jo et al., 2003; Jo et al., 2005; Buxbaum, 2004), and CREB/CREM transcription factors (Ledo et al., 2000; Ledo et al., 2002).

The role of $\mathrm{Ca}^{2+}$ in regulating DREAM functions and the structural basis of DREAM interactions with multiple partners are not fully understood. Although the solution structure of $\mathrm{Ca}^{2+}$ bound DREAM (residues 76 - 256) is known (Lusin et al., 2008) as well as the solution structure of the C-terminal domain (residues $161-256$ ) of $\mathrm{Ca}^{2+}$ bound DREAM (Yu et al., 2007), it remains unclear how the $\mathrm{Ca}^{2+} / \mathrm{Mg}^{2+}$ association to the EFhands alters the structural properties of DREAM. Considering the large number of DREAM interacting proteins, understanding the structural diversity could provide important insight into the relationship between the sequence, structure, and target diversity of DREAM and NCS proteins in general. Since the unique tryptophan (W169) positions between the N- and C-terminal hydrophobic clusters in DREAM (Figure 4.1) is ideal for monitoring conformational response to changes in $\mathrm{Ca}^{2+} / \mathrm{Mg}^{2+}$ concentration, I have combined time-resolved fluorescence, CD spectroscopy, and molecular dynamics 
simulations to probe the dynamics and structural diversity of DREAM under various conditions. The heterogeneous W169 emission decay indicates that DREAM adopts multiple conformations in the apo and $\mathrm{Ca}^{2+}$ bound forms. $\mathrm{Ca}^{2+}$ binding to EF-hands is associated with an increase in protein rigidity and a decrease of W169 solvent exposure. DREAM unfolds through a transition state that is populated at a $\mathrm{GuHCl}$ concentration around $4.5-5.0 \mathrm{M}$. The transition state is stabilized by $\mathrm{Ca}^{2+}$ binding to EF-hands 3 and 4 in DREAM. The presence of $\mathrm{Mg}^{2+}$ stabilizes the native structure of $\mathrm{Ca}^{2+}$ bound DREAM with respect to the transition state.

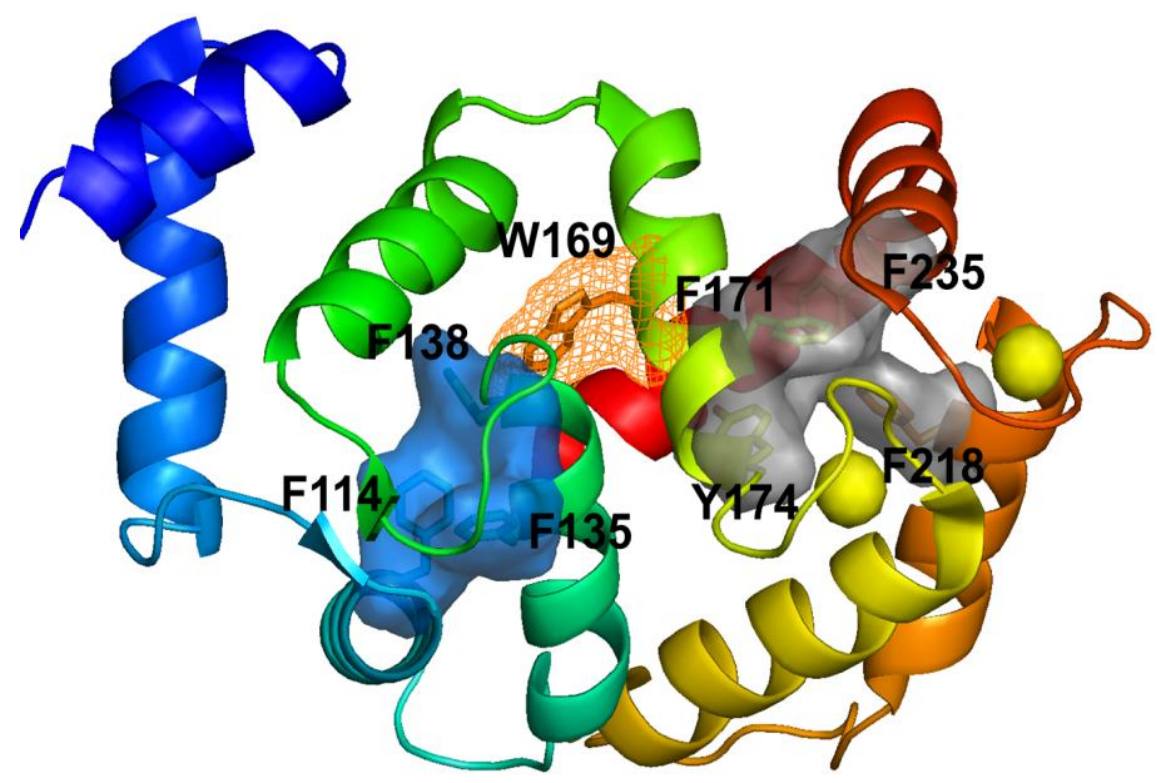

Figure 4.1: The aromatic clusters identified in DREAM C-terminus (grey solid surface) and $\mathrm{N}$-terminus (blue solid surface). EF-hand 1 in DREAM is shown in cyan, EF-hand 2 in green, EF-hand 3 in yellow, EF-hand 4 in orange, W169 in wireframe surface, and calcium ions shown as yellow spheres 


\subsection{Results}

\subsubsection{Steady-state emission spectra}

The emission spectra of ApoDREAM and $\mathrm{Mg}^{2+}$ DREAM are almost identical, and are broad with a maximum at $340 \mathrm{~nm}$ (Figure 4.2). Upon $\mathrm{Ca}^{2+}$ association to DREAM or $\mathrm{Mg}^{2+}$ DREAM, I observed that the two spectra change in the same manner with a decrease in the emission intensity (-18\% relative to that of $\mathrm{Apo}$ or $\left.\mathrm{Mg}^{2+} \mathrm{DREAM}\right)$ along with a concomitant hypsochromic shift of $5 \mathrm{~nm}$. Analogous $\mathrm{Ca}^{2+}$ triggered bathochromic shifts were reported previously, although the emission spectra presented here are approximately $5 \mathrm{~nm}$ blue shifted compared to the published results (Osawa et al., 2001). The protein was purified and studied in the presence of LDAO since the previous study had shown that the addition of LDAO reduces protein aggregation and stabilizes ApoDREAM and $\mathrm{Ca}^{2+}$ DREAM in its tetrameric and dimeric form, respectively (Osawa et al., 2001). I have observed that the presence of LDAO strongly affects the emission spectra of $\mathrm{Ca}^{2+}$ free and $\mathrm{Ca}^{2+}$ bound DREAM. For example, DREAM samples dialyzed against $20 \mathrm{mM}$ Tris buffer, $1 \mathrm{mM}$ DTT, and $10 \mathrm{mM}$ LDAO at $\mathrm{pH} 7.4$ for 48 hours provided emission spectra with a $\lambda_{\max }$ of $340 \mathrm{~nm}$ and $335 \mathrm{~nm}$ for Apo and $\mathrm{Ca}^{2+}$ DREAM, respectively, which are comparable to those reported by Osawa et al. (Osawa et al. 2001). On the other hand, emission spectra of DREAM samples prepared in the absence of LDAO are blue shifted with an emission maximum at $~ 330 \mathrm{~nm}$ for $\mathrm{Ca}^{2+}$ DREAM and $335 \mathrm{~nm}$ for ApoDREAM suggesting that LDAO binding to DREAM increases the solvent exposure of the W169 side-chain. The fluorescence quantum yield was determined to be $0.105 \pm 0.009$ for Apo and $\mathrm{Mg}^{2+} \mathrm{DREAM}$ and $0.090 \pm 0.011$ in $\mathrm{Ca}^{2+}$ and $\mathrm{Ca}^{2+} \mathrm{Mg}^{2+}$ DREAM. The bathochromic shift of the W169 emission maximum suggest that $\mathrm{Ca}^{2+}$ association to DREAM leads to a conformational 
transition upon which the W169 residue moves towards the hydrophobic core of the protein.

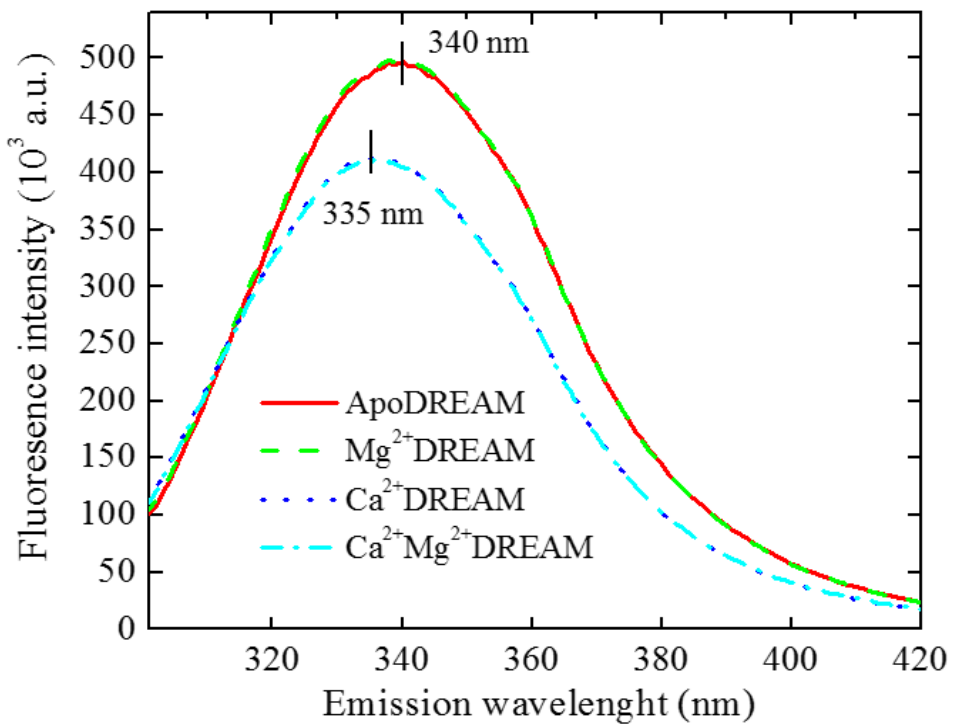

Figure 4.2: Fluorescence emission spectra of $\mathrm{Apo}, \mathrm{Mg}^{2+}, \mathrm{Ca}^{2+}$, and $\mathrm{Ca}^{2+} \mathrm{Mg}^{2+} \mathrm{DREAM}$ upon excitation at $295 \mathrm{~nm}$. Note that the emission spectrum of ApoDREAM and $\mathrm{Mg}^{2+}$ DREAM are almost identical, and likewise for $\mathrm{Ca}^{2+}$ DREAM and $\mathrm{Ca}^{2+} \mathrm{Mg}^{2+}$ DREAM.

\subsubsection{Fluorescence quenching}

Acrylamide, a polar, uncharged water-soluble molecule, was used in quenching studies to further probe the solvent accessibility of W169 in DREAM. The plot of the ratio of the DREAM fluorescence emission intensities in the absence and presence of the acrylamide quencher exhibits a linear dependence (Figure 4.3).

The Stern-Volmer constants were determined using equation 3.22 in section 3.2.5.2.4. The determined $\mathrm{K}_{\mathrm{sv}}$ constants are $2.80 \pm 0.01 \mathrm{M}^{-1}$ for ApoDREAM, $2.03 \pm$ $0.10 \mathrm{M}^{-1}$ for $\mathrm{Ca}^{2+} \mathrm{DREAM}$, and $1.78 \pm 0.01 \mathrm{M}^{-1}$ for $\mathrm{Ca}^{2+} \mathrm{Mg}^{2+}$ DREAM, confirming that the W169 side chain is less solvent accessible in the structure of $\mathrm{Ca}^{2+}$ bound DREAM. In the case of collisional quenching, the bimolecular quenching rate constant, $\mathrm{k}_{\mathrm{q}}=\mathrm{K}_{\mathrm{Sv}} /\langle\tau\rangle$, 
where $\langle\tau\rangle$ is the lifetime in the absence of the quencher, provides a better estimate of the quenching efficiency.

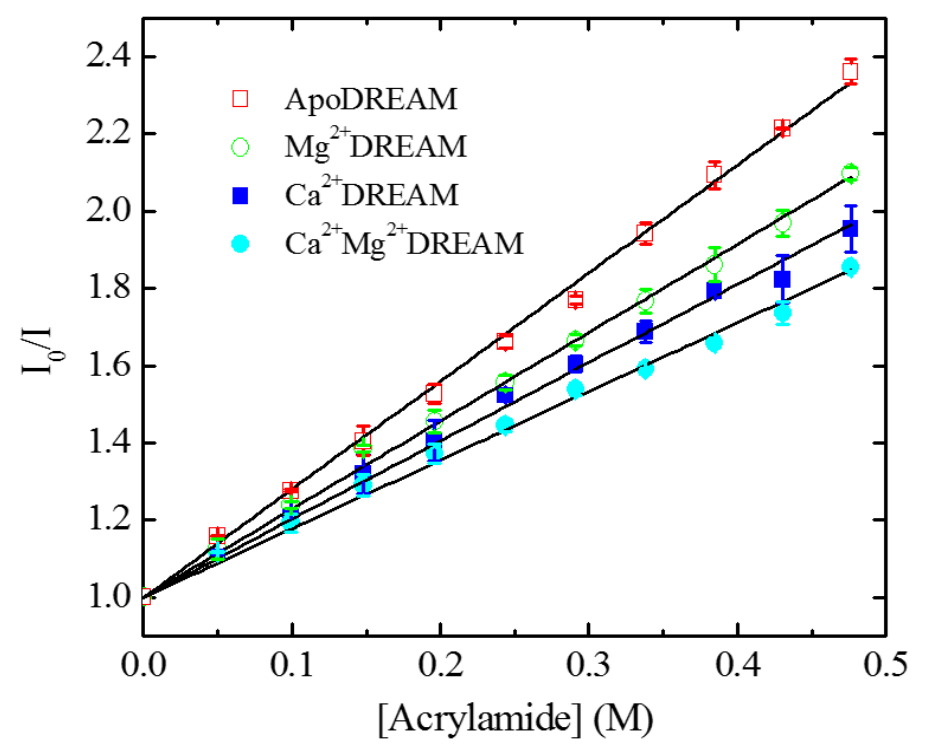

Figure 4.3: Stern-Volmer plot for acrylamide quenching of DREAM in the absence/presence of $\mathrm{Ca}^{2+}$ and $\mathrm{Mg}^{2+}$. Error bar represents standard deviations of three independent measurements.

Using the average value for the fluorescence lifetime (Table 4.2), the $\mathrm{k}_{\mathrm{q}}$ value was determined to be $6.2 \times 10^{8} \mathrm{M}^{-1} \mathrm{~s}^{-1}$ for ApoDREAM, $4.2 \times 10^{8} \mathrm{M}^{-1} \mathrm{~s}^{-1}$ for $\mathrm{Ca}^{2+}$ DREAM, and $3.7 \times 10^{8} \mathrm{M}^{-1} \mathrm{~s}^{-1}$ for $\mathrm{Ca}^{2+} \mathrm{Mg}^{2+} \mathrm{DREAM}$ (Table 4.1). Interestingly, $\mathrm{Mg}^{2+}$ association to DREAM results in a small but reproducible decrease in the Stern-Volmer constant and the bimolecular quenching constant, $\mathrm{K}_{\mathrm{sv}}=2.29 \pm 0.06 \mathrm{M}^{-1}$ and $\mathrm{k}_{\mathrm{q}}=5.0 \times 10^{8} \mathrm{M}^{-1} \mathrm{~s}^{-1}$ (Table 4.1), indicating that $\mathrm{Mg}^{2+}$ binding to EF-hand 2 causes a minor structural reorganization that impacts the W169 solvent accessibility. These quenching constants are listed in Table 4.1. Previously, I have shown that $\mathrm{Mg}^{2+}$ binding to DREAM leads to small changes in the fluorescence properties of 1,8-ANS DREAM complexes (Gonzalez et al., 2014), supporting the role of $\mathrm{Mg}^{2+}$ in stabilizing the tertiary structure of the DREAM protein. 
Table 4.1: Stern-Volmer constant $\left(\mathrm{K}_{\mathrm{sv}}\right)$ and bimolecular quenching constant $\left(\mathrm{k}_{\mathrm{q}}\right)$ of DREAM protein

\begin{tabular}{|l|c|c|}
\hline \multicolumn{1}{|c|}{ Protein } & $\mathrm{K}_{\mathrm{sv}}\left(\mathrm{M}^{-1}\right)$ & $\mathrm{k}_{\mathrm{q}}\left(\mathrm{x} 10^{8} \mathrm{M}^{-1} \mathrm{~s}^{-1}\right)$ \\
\hline ApoDREAM & 2.80 & 6.2 \\
\hline $\mathrm{Mg}^{2+} \mathrm{DREAM}$ & 2.29 & 5.0 \\
\hline $\mathrm{Ca}^{2+} \mathrm{DREAM}^{2+} \mathrm{Cg}^{2+} \mathrm{DREAM}$ & 2.03 & 4.2 \\
\hline $\mathrm{Ca}^{2+} \mathrm{Mg}^{-1}$ & 1.78 & 3.7 \\
\hline
\end{tabular}

\subsubsection{Fluorescence lifetime}

Since the tryptophan fluorescence lifetime is highly sensitive to small variations in the fluorophore environment, probing the lifetime of the single W169 residue in DREAM provides further insight into $\mathrm{Mg}^{2+} / \mathrm{Ca}^{2+}$ induced conformational changes, including alteration of local dynamics. The W169 lifetime was determined using phase modulation fluorescence spectroscopy. The phase shift and modulation ratio data were monitored in the frequency range from $5 \mathrm{MHz}$ to $250 \mathrm{MHz}$ and are presented in Figure 4.4.

Fluorescence lifetime data were analyzed using a combination of discrete exponentials and various distribution models (Gaussian, Lorentzian or uniform). The best fits based on residuals and $\chi^{2}$ values were obtained with a model composed of a discrete single exponential decay component and a continuous Gaussian distribution (Table 4.2). The discrete component exhibits a calcium independent lifetime of $\sim 7.5 \mathrm{~ns}$ whereas the Gaussian distribution is centered at $3.5 \mathrm{~ns}$ and $1.7 \mathrm{~ns}$ for $\mathrm{Ca}^{2+}$ free and $\mathrm{Ca}^{2+}$ bound DREAM, respectively. The width of the distribution is $\mathrm{w}_{1}=1.8 \mathrm{~ns}$ and $2.0 \mathrm{~ns}$ in the ApoDREAM and $\mathrm{Mg}^{2+}$ DREAM, respectively, and narrows upon $\mathrm{Ca}^{2+}$ binding to $\mathrm{w}_{1}=0.8 \mathrm{~ns}$ and $0.9 \mathrm{~ns}$ in $\mathrm{Ca}^{2+} \mathrm{DREAM}$ and $\mathrm{Ca}^{2+} \mathrm{Mg}^{2+} \mathrm{DREAM}$, respectively (Figure 4.5). In addition, the 
fractional contribution of the $7.5 \mathrm{~ns}$ component is $\sim 0.12$ in the Apo and $\mathrm{Mg}^{2+}$ DREAM and increases to 0.20 in $\mathrm{Ca}^{2+}$ bound forms of DREAM.

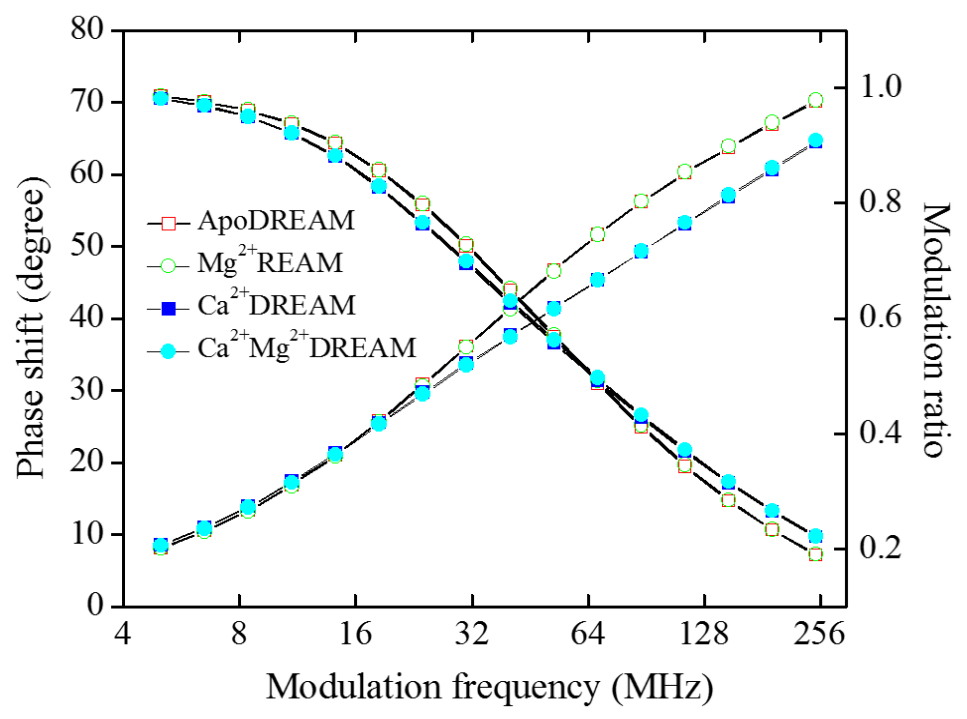

Figure 4.4: Frequency-domain intensity decay of $40 \mu \mathrm{M}$ ApoDREAM (open squares), $\mathrm{Mg}^{2+}$ DREAM (open circles), $\mathrm{Ca}^{2+}$ DREAM (closed squares), and $\mathrm{Mg}^{2+} \mathrm{Ca}^{2+}$ DREAM (closed circles). The solid lines represent the data fitting using a Gaussian distribution model and single exponential decay model. Note that the experimental data for ApoDREAM and $\mathrm{Mg}^{2+}$ DREAM are nearly identical, and likewise for the data for $\mathrm{Ca}^{2+}$ DREAM and $\mathrm{Ca}^{2+} \mathrm{Mg}^{2+}$ DREAM.

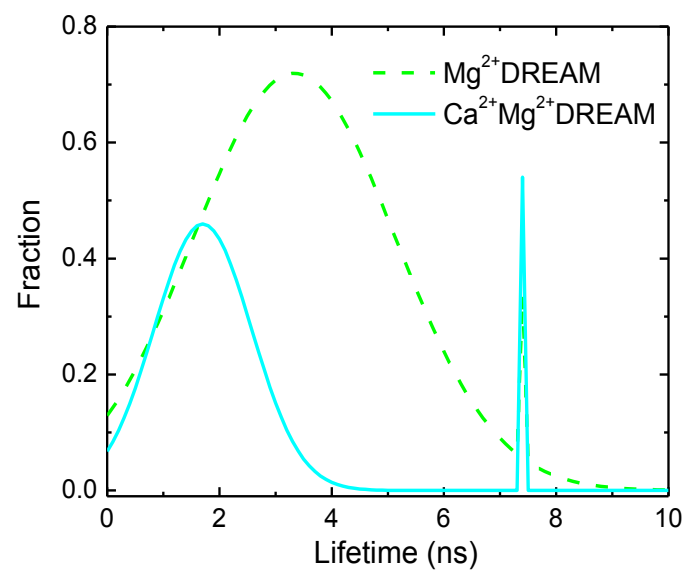

Figure 4.5: Fluorescence lifetime rotational distribution of $\mathrm{Mg}^{2+}$ DREAM and $\mathrm{Ca}^{2+} \mathrm{Mg}^{2+}$ DREAM using a continuous Gaussian distribution and a discrete single exponential decay component. To simplify, the lifetime rotational distribution curves of ApoDREAM and $\mathrm{Ca}^{2+}$ DREAM are not shown. 
Table 4.2: Emission decay parameters for DREAM using a continuous Gaussian distribution and a discrete component

\begin{tabular}{|l|c|c|c|c|c|c|c|c|c|}
\hline \multicolumn{1}{|c|}{ Protein } & $\begin{array}{c}\tau_{1} \\
(\mathrm{~ns})\end{array}$ & $\begin{array}{c}\mathrm{w}_{1} \\
(\mathrm{~ns})\end{array}$ & $f_{1}$ & $\alpha_{1}$ & $\begin{array}{c}\tau_{2} \\
(\mathrm{~ns})\end{array}$ & $f_{2}$ & $\alpha_{2}$ & $\begin{array}{c}\langle\tau\rangle \\
(\mathrm{ns})\end{array}$ & $\chi^{2}$ \\
\hline ApoDREAM & 3.6 & 2.0 & 0.77 & 0.88 & 7.5 & 0.23 & 0.12 & 4.5 & 1.7 \\
\hline $\mathrm{Mg}^{2+} \mathrm{DREAM}$ & 3.4 & 1.8 & 0.75 & 0.87 & 7.5 & 0.25 & 0.13 & 4.4 & 2.0 \\
\hline $\mathrm{Ca}^{2+} \mathrm{DREAM}$ & 1.8 & 0.8 & 0.44 & 0.78 & 7.4 & 0.56 & 0.22 & 4.8 & 1.7 \\
\hline $\mathrm{Ca}^{2+} \mathrm{Mg}^{2+} \mathrm{DREAM}$ & 1.8 & 0.9 & 0.48 & 0.80 & 7.6 & 0.52 & 0.20 & 4.8 & 1.0 \\
\hline
\end{tabular}

$\tau_{1}$ is the mean decay time of the Gaussian distribution with a width of distribution $w_{1}$. $\tau_{2}$ is the lifetime of the discrete single exponential term. The average lifetime $\langle\tau\rangle$ was calculated using equations 3.54 and 3.55 in Method section 3.2.10.3. Parameters $\alpha_{1}$ and $\alpha_{2}$ are normalized pre-exponential decay and $f_{1}$ and $f_{2}$ are exponential decay fractions.

\subsubsection{CD measurements}

The far-UV CD spectra of DREAM show the characteristic of alpha-helical proteins, with negative peaks at $222 \mathrm{~nm}$ and $208 \mathrm{~nm}$ (Figure 4.6). The magnitudes of the CD signals of ApoDREAM and $\mathrm{Mg}^{2+}$ DREAM are identical. However, when $\mathrm{Ca}^{2+}$ is bound to Apo and $\mathrm{Mg}^{2+} \mathrm{DREAM}$, magnitude of CD signal becomes more negative.

These results suggest that binding of $\mathrm{Ca}^{2+}$ to DREAM increases the alpha-helical content of the protein. Analysis of the far-UV CD spectra of DREAM using K2D protein secondary prediction software shows that DREAM protein increases about $16 \%$ of its alpha-helical content in the $\mathrm{Ca}^{2+}$ bound form relative to that of $\mathrm{Apo}$ and $\mathrm{Mg}^{2+}$ forms. 


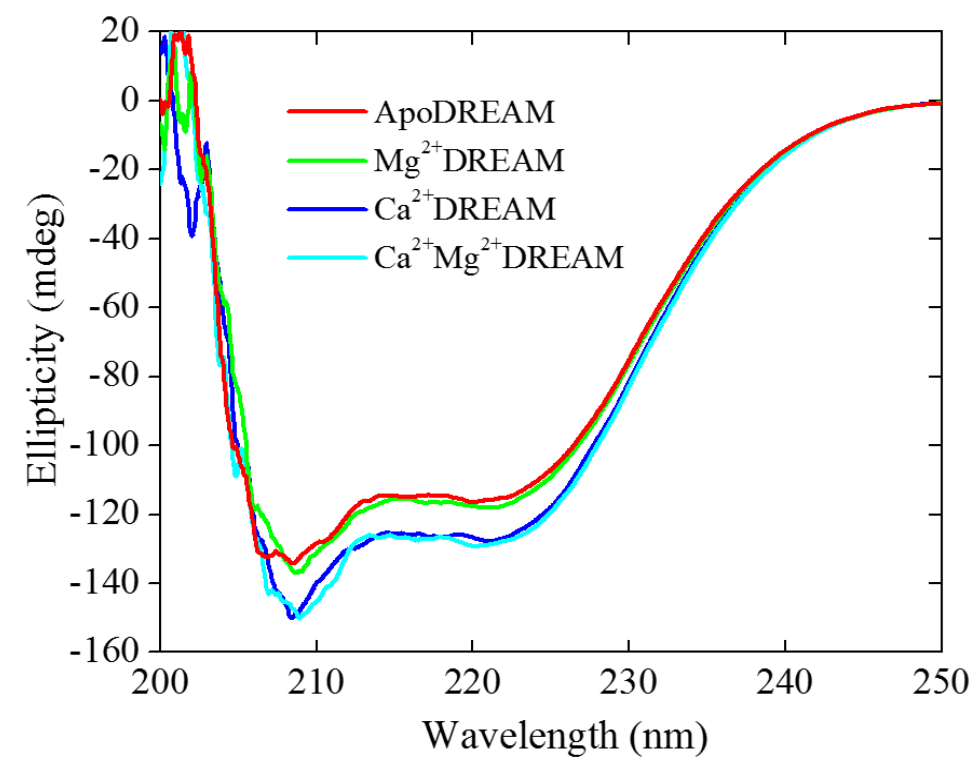

Figure 4.6: CD spectra of Apo, $\mathrm{Mg}^{2+}, \mathrm{Ca}^{2+}$, and $\mathrm{Ca}^{2+} \mathrm{Mg}^{2+}$ DREAM in the far-UV region. The concentration of the protein was $40 \mu \mathrm{M}$ in $10 \mathrm{mM}$ Tris buffer (pH 7.4), $1 \mathrm{mM}$ DTT, and $10 \mathrm{mM}$ LDAO. Spectra were corrected for the baseline of the buffer.

\subsubsection{DREAM stability}

The impact of $\mathrm{Ca}^{2+} / \mathrm{Mg}^{2+}$ binding to EF-hands on the protein stability was characterized by monitoring the $\mathrm{CD}$ signal at $220 \mathrm{~nm}$ as a function of increasing $\mathrm{GuHCl}$ concentration as shown in Figure 4.7 (a). The unfolding curves, shown in Figure 4.7 (b), clearly indicate the presence of a partially folded intermediate at $\mathrm{GuHCl}$ concentrations between 4.0 and 5.0 M. The experimental data were analyzed using a three-state model according to equation 3.60 in section 3.2.3.4 and the recovered thermodynamic parameters $\left(\Delta \mathrm{G}^{\circ}, \mathrm{m}\right.$, and $\left.\mathrm{C}_{\mathrm{m}}\right)$ are summarized in Table 4.2. $\mathrm{Ca}^{2+}$ association to DREAM increases the stability of the native state compared to the unfolded state by $\sim 4 \mathrm{kcal} \mathrm{mol}^{-1}$, whereas the contribution of $\mathrm{Mg}^{2+}$ to DREAM stability is significantly smaller. Interestingly, $\mathrm{Ca}^{2+}$ binding to EF-hands increases the stability of the native state with respect to the partially unfolded intermediate state by $\sim 2 \mathrm{kcal} \mathrm{mol}^{-1}$ in the presence of $\mathrm{Mg}^{2+}$, whereas the stability 
of the intermediate state with respect to the unfolded state is increased in both $\mathrm{Ca}^{2+} \mathrm{DREAM}$ and $\mathrm{Ca}^{2+} \mathrm{Mg}^{2+}$ DREAM by $\sim 2 \mathrm{kcal} \mathrm{mol}^{-1}$. The shallow folding transition between the native and partially unfolded intermediate state points to a low energy barrier between the native and intermediate state for Apo, $\mathrm{Mg}^{2+}$, and $\mathrm{Ca}^{2+} \mathrm{DREAM}$. The native state is more strongly stabilized with respect to the intermediate state in $\mathrm{Ca}^{2+} \mathrm{Mg}^{2+} \mathrm{DREAM}$, as evident from the steep transition between the folded and partially folded intermediate state.
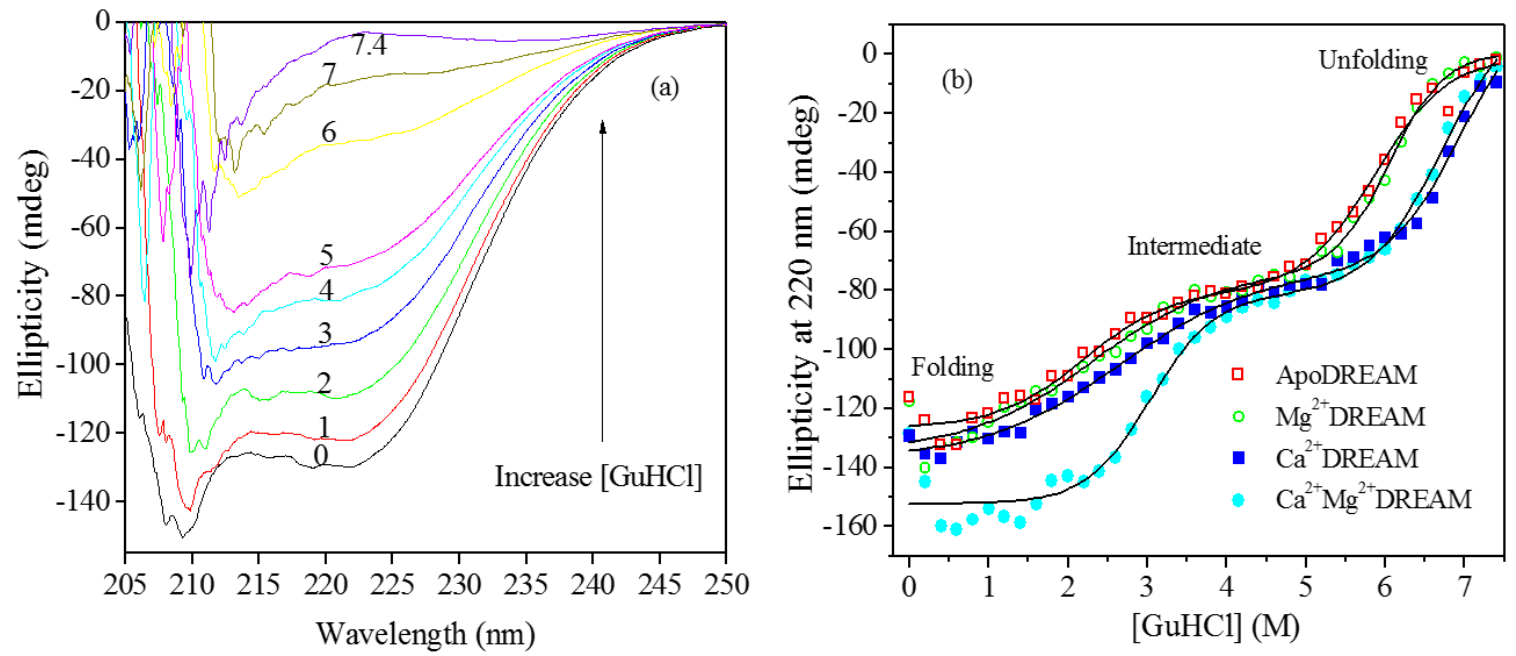

Figure 4.7: (a): CD spectra of $40 \mu \mathrm{M}$ ApoDREAM in the presence of $0,1,2,3,4,5,6,7$, and $7.4 \mathrm{M} \mathrm{GuHCl}$. (b): Representative equilibrium unfolding traces of DREAM as determined by the $C D$ signal change at $220 \mathrm{~nm}$. Solid black lines are fitting curves of experimental data using equation 3.60 in section 3.2.10.4. The thermodynamic parameters are reported in Table 4.3 . 
Table 4.3: Thermodynamic parameters for $\mathrm{GuHCl}$ induced denaturation of DREAM in the absence/presence of $\mathrm{Ca}^{2+}$ and $\mathrm{Mg}^{2+}$

\begin{tabular}{|c|c|c|c|c|c|c|c|}
\hline Protein & $\begin{array}{c}\Delta \mathrm{G}_{N \rightarrow I} \\
\text { kcal mol }^{-1}\end{array}$ & $\begin{array}{c}\mathrm{m}_{N \rightarrow I} \\
\text { kcal } M^{-1} \mathrm{~mol}^{-1}\end{array}$ & $\begin{array}{c}\mathrm{C}_{N \rightarrow I} \\
M\end{array}$ & $\begin{array}{c}\Delta \mathrm{G}_{I \rightarrow U} \\
\text { kcal mol }^{-1}\end{array}$ & $\begin{array}{c}\mathrm{m}_{I \rightarrow U} \\
\text { kcal } M^{-1} \mathrm{~mol}^{-1}\end{array}$ & $\begin{array}{c}\mathrm{C}_{I \rightarrow U} \\
M\end{array}$ & $\begin{array}{c}\Delta \mathrm{G}_{N \rightarrow U} \\
\text { kcal mol }^{-1}\end{array}$ \\
\hline ApoDREAM & $1.6 \pm 0.1$ & $0.8 \pm 0.1$ & 2.0 & $7.7 \pm 0.8$ & $1.3 \pm 0.1$ & 5.9 & $9.3 \pm 0.8$ \\
\hline $\mathrm{Mg}^{2+} \mathrm{DREAM}$ & $1.7 \pm 0.2$ & $0.8 \pm 0.1$ & 2.1 & $8.4 \pm 0.9$ & $1.4 \pm 0.2$ & 6.0 & $10.1 \pm 0.9$ \\
\hline $\mathrm{Ca}^{2+}$ DREAM & $1.6 \pm 0.3$ & $0.6 \pm 0.1$ & 2.0 & $11.3 \pm 0.1$ & $1.7 \pm 0.1$ & 6.5 & $12.9 \pm 0.3$ \\
\hline $\mathrm{Ca}^{2+} \mathrm{Mg}^{2+}$ DREAM & $3.8 \pm 0.4$ & $1.2 \pm 0.2$ & 3.2 & $10.5 \pm 1.2$ & $1.6 \pm 0.2$ & 6.5 & $14.3 \pm 1.3$ \\
\hline
\end{tabular}

The thermodynamic parameters were calculated using equation 3.60 in section 3.2.10.4 where N, I, and U correspond to the native state, transition state, and unfolded state, respectively. $\Delta \mathrm{G}$ is the standard free energy for the transition, $\mathrm{m}$ is the dependence of the free energy on the denaturant concentration, and $\mathrm{C}_{\mathrm{N} \rightarrow \mathrm{I}}$ and $\mathrm{C}_{\mathrm{N} \rightarrow U} \mathrm{U}$ represent denaturant concentration of half unfold from native to transition state and from transition state to unfolded state, respectively. $\mathrm{G}_{N \rightarrow U}$ represents the overall unfolding free energy from native to unfolded state. Errors were determined from standard deviations of at least three independent measurements. 


\subsubsection{Molecular dynamics studies}

Molecular dynamics simulations provide a complementary approach to monitor structural transitions in DREAM. Molecular dynamics simulations of Apo and $\mathrm{Ca}^{2+}$ bound DREAM were performed in collaboration with Dr. Prem Chapagain (Department of Physics, Florida International University). The root mean square fluctuation (RMSF) of $\mathrm{C}_{\alpha}$ atoms can be used to probe fluctuations of individual residues with respect to the average structure in the Apo and $\mathrm{Ca}^{2+}$ bound form of DREAM. The RMSF profile for a $40 \mathrm{~ns}$ time window is shown in Figure 4.8. The overall profiles are similar for Apo and $\mathrm{Ca}^{2+}$ bound DREAM. $\mathrm{Ca}^{2+}$ association to EF-hands 3 and 4 reduces the fluctuations of the $\mathrm{Ca}^{2+}$ binding loop in EF-hand 3 (residues 174 to 183), and to a smaller extent reduces the fluctuations in the binding loop of EF-hand 4 (residues 222 to 231). The flexibility of the long loop connecting $\alpha$-helix 7 to $\alpha$-helix 8 (residues 198 to 211 ) remains high in the $\mathrm{Ca}^{2+}$ bound form of the protein, in agreement with NMR results that show increased structural deviations in this region (Lusin et al., 2008). Interestingly, the C- terminal loop (residues 242 to 244 ) connecting $\alpha$-helix 9 and $\alpha$-helix 10 in the C-terminal domain exhibits high

flexibility in the $\mathrm{Ca}^{2+}$ bound structure. As expected, smaller changes in protein flexibility due to $\mathrm{Ca}^{2+}$ binding to DREAM are observed for the $\mathrm{N}$-terminal domain of the protein. 


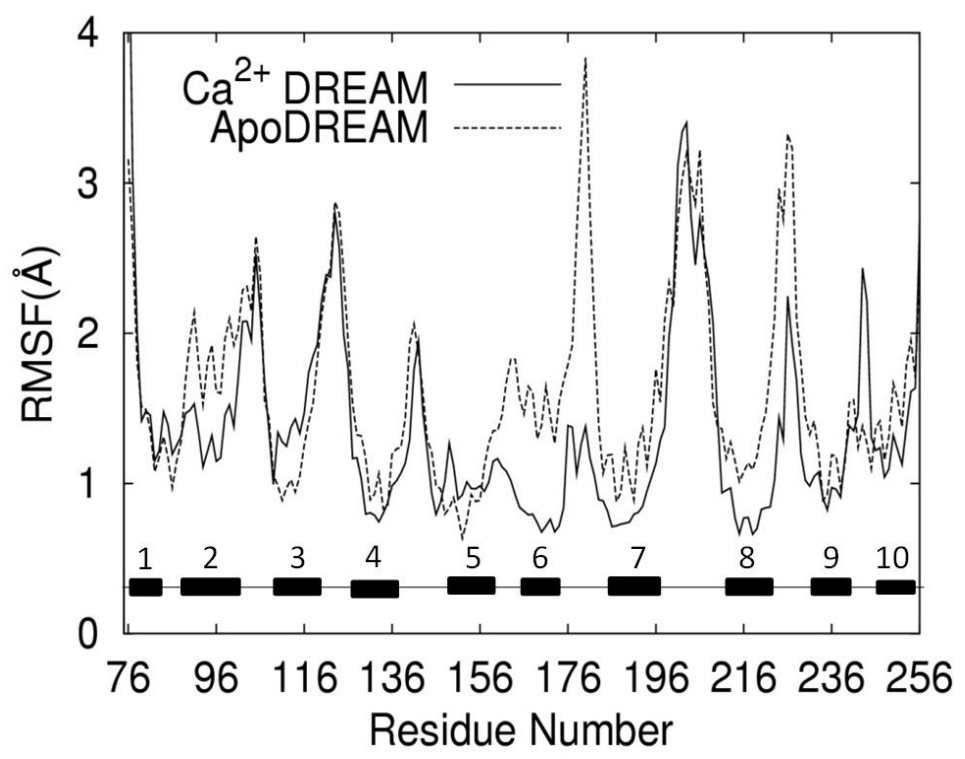

Figure 4.8: Root-mean-square fluctuations (RMSF) of the $\mathrm{C}_{\alpha}$ backbone in ApoDREAM and $\mathrm{Ca}^{2+}$ DREAM.

The superposition of MD simulation structures of ApoDREAM and $\mathrm{Ca}^{2+}$ DREAM monomers (Figure 4.9) shows high structural similarity. Detailed inspection of these structures reveals that the $\mathrm{N}$-terminal domain containing the non-functional EF-hand 1 and EF-hand 2 does not show significant structural differences between the Apo and $\mathrm{Ca}^{2+}$ bound forms apart from $\alpha$-helix 2, which moves away from $\alpha$-helix 5 in the $\mathrm{Ca}^{2+}$ bound protein. A larger structural reorganization is observed for the C-terminal domain and includes repositioning of the $\alpha$-helices within the individual EF-hand binding sites. Interestingly, the entering and exiting helices of the EF-hand 3 undergo a minor reorientation in the calcium bound state with respect to the Apostate, whereas $\mathrm{Ca}^{2+}$ association to EF-hand 4 is accompanied by the clear transition of the $\alpha$-helices from a perpendicular to a parallel orientation (Figure 4.9). Also, the C-terminal helix ( $\alpha$-helix 10) moves away from $\alpha$-helix 6 in the $\mathrm{Ca}^{2+}$ bound protein. 

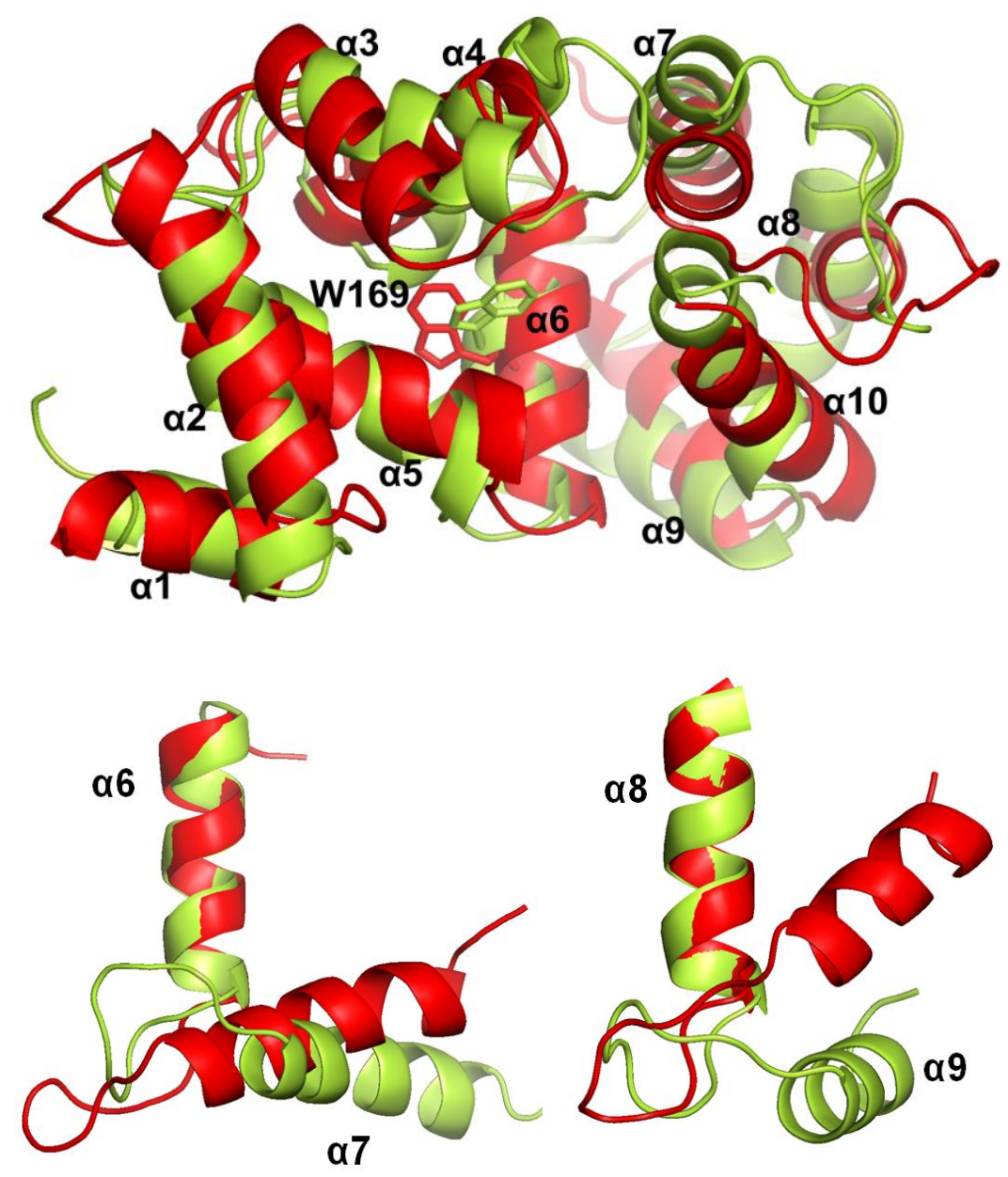

Figure 4.9: Top panel: Overlaid MD simulations structures of ApoDREAM (in red) and $\mathrm{Ca}^{2+}$ bound DREAM (in yellow). Alpha-helices $1-10$ in DREAM are labeled by $\alpha 1-\alpha 10$, respectively. EF-hand 1 is composed of $\alpha 2$ and $\alpha 3$, EF-hand 2 composed of $\alpha 4$ and $\alpha 5$, EFhand 3 composed of $\alpha 6$ and $\alpha 7$, EF-hand 4 composed of $\alpha 8$ and $\alpha 9$. The position of the W169 residue is shown with sticks. Bottom panel: $\mathrm{Ca}^{2+}$ triggered reorganization of the EFhand 3 (left) and EF-hand 4 (right).

\subsection{Discussion}

Previous studies have demonstrated that $\mathrm{Ca}^{2+}$ association to EF-hands 3 and 4 in the C-terminal domain of DREAM leads to the dissociation of the ApoDREAM tetramer into dimers, which decreases DREAM affinity for intracellular partners (Osawa et al., 2005), as well as DREAM monomer affinity for small hydrophobic molecules (Gonzalez 
and Miksovska, 2014). However, the detailed molecular mechanism of how $\mathrm{Ca}^{2+}$ association to the C-terminal domain EF-hands modulates the conformation of the DREAM monomer and the impact of $\mathrm{Ca}^{2+} / \mathrm{Mg}^{2+}$ association on DREAM affinity for intracellular partners remains elusive. Tryptophan emission has been widely used as a sensitive probe of structural transitions in proteins including protein folding, $\mathrm{Ca}^{2+} / \mathrm{Mg}^{2+}$ association to proteins and protein-protein interactions. In the DREAM structure (residues 65 to 256 ), the single $\mathrm{W} 169$ residue that is located at the interface between the $\mathrm{N}$ - and Cterminal domains provides a unique tool for characterization of $\mathrm{Ca}^{2+} / \mathrm{Mg}^{2+}$ triggered conformational dynamics of DREAM.

\subsubsection{Impact of $\mathrm{Ca}^{2+}$ binding on conformational dynamics of DREAM}

Steady-state emission data of W169 indicate that $\mathrm{Ca}^{2+}$ association to EF-hand 3 and EF-hand 4 triggers the reorganization of the interface between EF-hand 2 and EF-hand 3 with the W169 side chain being located in a less polar environment in the $\mathrm{Ca}^{2+}$ bound form. This is further supported by the emission quenching and fluorescence lifetime data. The smaller bimolecular quenching rate constant observed for $\mathrm{Ca}^{2+}$ and $\mathrm{Ca}^{2+} \mathrm{Mg}^{2+}$ DREAM is consistent with a reduced solvent accessibility to the W169 residue due to tertiary structure alterations and/or decreased conformational flexibility in the $\mathrm{Ca}^{2+}$ bound form of DREAM. The decrease in the structural flexibility is supported by the W169 lifetime data (Figure 4.4). The major fraction of the W169 emission decay can be described using a continuous Gaussian distribution model centered at $3.5 \mathrm{~ns}$ for $\mathrm{Apo}$ and $\mathrm{Mg}^{2+}$ DREAM and $1.7 \mathrm{~ns}$ for $\mathrm{Ca}^{2+}$ DREAM and $\mathrm{Ca}^{2+} \mathrm{Mg}^{2+}$ DREAM. The minor fraction $(\sim 10 \%$ in ApoDREAM and 20 $\%$ in $\mathrm{Mg}^{2+}$ and/or $\mathrm{Ca}^{2+}$ bound DREAM) exhibits a discrete single exponential decay with lifetime of $\sim 7.5 \mathrm{~ns}$. The full width at half maximum value of the lifetime distribution 
parameter, $w$, was found to be $2.0 \mathrm{~ns}$ for Apo and $\mathrm{Mg}^{2+}$ DREAM and $\sim 0.9 \mathrm{~ns}$ for $\mathrm{Ca}^{2+}$ bound DREAM. The observed decrease in the $w$ value suggests that $\mathrm{Ca}^{2+}$ binding to EFhands reduces the distribution of conformational microstates and/or modulates the dynamics of the inter-conversion between individual sub-states in DREAM (Alcala et al., 1987; Bodis et al., 2013). Several mechanisms were proposed to explain the observed heterogeneity of the tryptophan lifetime in single tryptophan proteins including the presence of tryptophan side chain rotamers, quenching by water molecules, electron transfer to the peptide carbonyl group, excited state electron or proton transfer, and intersystem crossing (Szabo and Rayner, 1980; Moncrieffe et al., 2000; Ross and Jameson, 2008). Interestingly, an overlay of the 15 lowest energy structures of $\mathrm{Ca}^{2+}$ bound DREAM determined by NMR spectroscopy (Lusin et al., 2008) and the structure of the DREAM Cterminal domain (residues 161-256) (Yu et al., 2007; Bodis et al., 2013) displays the presence of a single tryptophan rotamer ( $\mathrm{t}$ rotamer) in the DREAM structure. However, as shown in Figure 4.9, a transition from the $\mathrm{t}$ to $\mathrm{g}+$ rotamer of W169 side chain is observed during molecular dynamic simulations, which supports the idea that the bimodal distribution could arise from two rotameric orientations of W169 with the side chain of the g+ rotamer being more solvent exposed.

Inspection of the DREAM and DREAM C-terminal domain structures (PDB entry 2JUL and 2E6W, respectively) reveals four charged amino acid residues located within 6 $\AA$ of the W169 indole ring that may serve as efficient quenchers for the $t$ rotamer. Residues E165, K168, and N172 are located on the same $\alpha$-helix as W169, whereas E253 is found at the end of $\alpha$-helix 10 (Figure 4.10). 

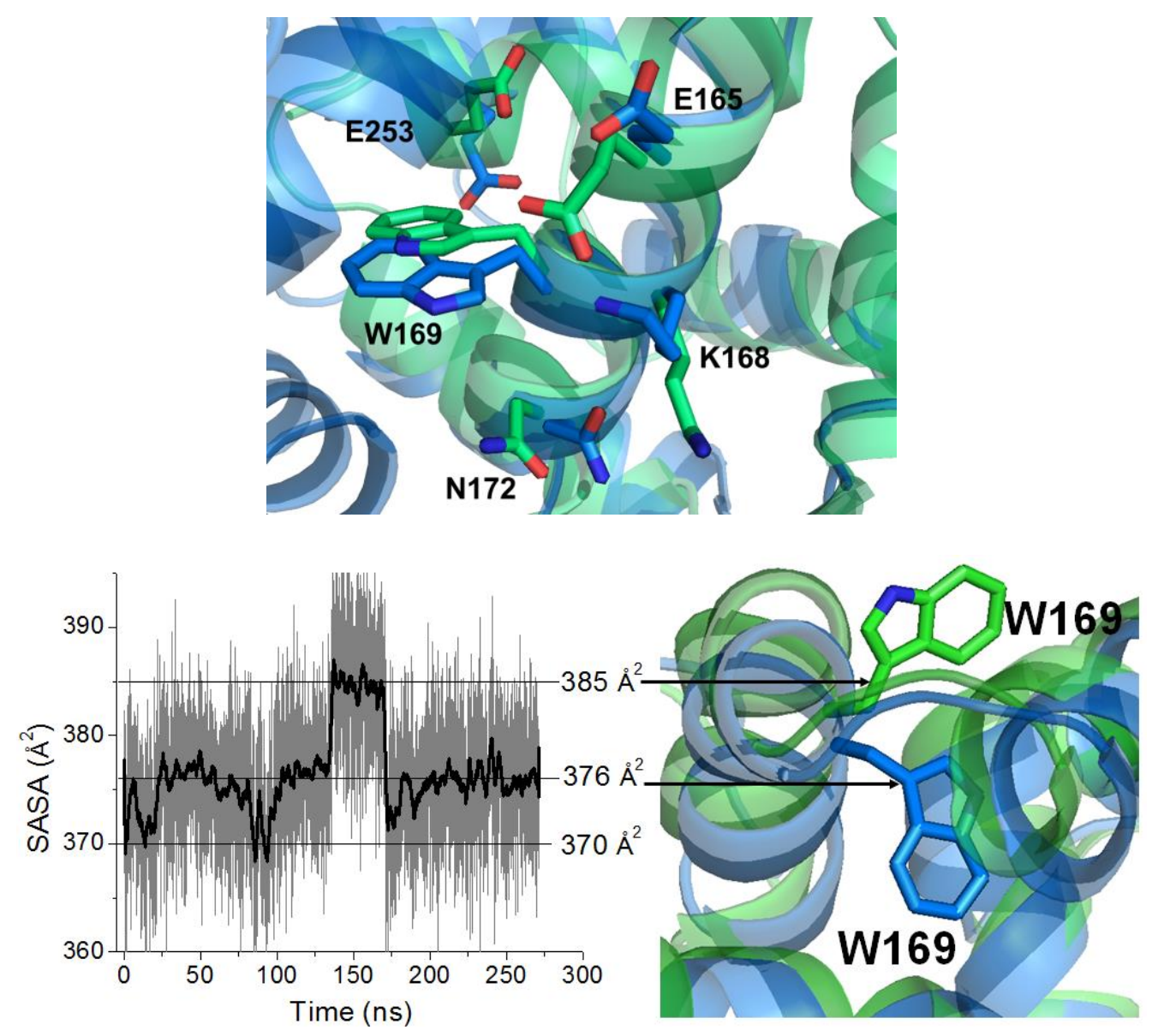

Figure 4.10: Top panel: charged and polar amino acid residues surrounding W169 in the structure of DREAM (PBD entry 2JUL, shown in blue) and C-terminal domain of DREAM (PBD entry 2E6W, shown in green). Bottom left panel: SASA of W169 in DREAM during 270 ns of the MD trajectory. Bottom right panel: Partially buried (in blue) and solvent exposed (in green) orientation of W169 side chain in DREAM structure.

In addition, the side chains of these residues are disordered in the NMR structures, which likely contribute to the observed heterogeneity of the W169 emission decay. In the absence of the NMR structure of ApoDREAM, it is difficult to pinpoint the origin of the slower W169 decay in Apo and $\mathrm{Mg}^{2+}$ DREAM. However, I speculate that the re-positioning of $\alpha$-helix 10 upon $\mathrm{Ca}^{2+}$ binding brings the E253 side chain closer to the W169 indole ring resulting in more efficient W169 emission quenching. Indeed, $\alpha$-helix 10 was found to be flexible in the structure of KChIP1 as it moves away from $\alpha$-helix 8 in the KChIP1:Kv4 
channel T1 domain complex (Wang et al., 2007). Also, the reorientation of $\alpha$-helix 10 was associated with increased affinity of $\mathrm{Ca}^{2+}$ bound DREAM for arachidonic acid and small hydrophobic molecules (Gonzalez and Miksovska, 2014).

The second W169 decay component is characterized by an unusually long lifetime of $\sim 7.5$ ns. It is unlikely that the presence of the second lifetime reflects other oligomerization forms of DREAM (monomer/tetramer in the case of $\mathrm{Ca}^{2+}$ free DREAM and monomer/dimer in the case of $\mathrm{Ca}^{2+}$ bound protein) since two comparable lifetimes were found for the C-terminal DREAM construct (residues 161-256) in the Apo form which does not form a tetramer or dimer in solution. On the other hand, a similar lifetime of $7.3 \mathrm{~ns}$ was detected in the multi-tryptophan protein beta-glycosidase from Sulfolobus solfataricus, and was associated with a rigid cluster of eight tryptophan residues (Bismuto, Nucci et al. 1999). However, the NMR structure (Figure 4.9, top) and RMSF from MD simulations (Figure 4.8) are not consistent with the presence of a rigid hydrophobic cluster surrounding W169 in DREAM. On the other hand, a single tryptophan residue in the calcium binding protein annexin was reported to exhibit two distinct lifetimes of $2.0 \mathrm{~ns}$ and $7.0 \mathrm{~ns}$ when annexin is associated to lipid vesicles (Follenius-Wund et al., 1997). The 7.0 ns decay was associated with the tryptophan side chain located in a more polar environment, at the interface between the protein surface and the lipid membrane (Follenius-Wund et al., 1997).

Thus, to further explore the dynamic behavior of the W169 side chain, a $250 \mathrm{~ns}$ molecular dynamics simulation was analyzed and the solvent accessible surface area (SASA) plot for W169 is shown in Figure 4.10. The W169 side chain samples three major conformations with distinct SASA values. The major and minor conformations are 
characterized by SASA values of $376 \AA^{2}$ and $370 \AA^{2}$, respectively, and can be attributed to the partially buried W169 side-chain within the interface between the C- and N-terminal domains of DREAM. The additional conformation with a SASA of $385 \AA^{2}$ corresponds to the solvent exposed indole ring (Figure 4.10). The binding site for the LDAO molecule on the DREAM surface is not known. The detergent molecule may bind into the hydrophobic cleft between the $\mathrm{N}$ - and $\mathrm{C}$-terminal domains in a similar way as a myristoyl group binds to ApoRecoverin protein. Thus, the interactions between the W169 indole ring and the polar head of the LDAO molecule bound to DREAM can contribute to a $7.5 \mathrm{~ns}$ W169 decay and the red-shifted emission spectra of DREAM samples prepared in the presence of LDAO. Furthermore, Liao et al. (Liao et al., 2009) have shown that the C-terminal domain of KChIP1 is required for KChIP1 association to phospholipid vesicles and the formation of KChIP1 - phospholipid vesicle complexes alters the protein structure. Taking into account a high sequence and structural homology of the C-terminal domain among the members of the KChIP subfamily, it is likely that DREAM forms similar complexes with phospholipid vesicles. Indeed, one can speculate that the DREAM conformation that is characterized by the $7.5 \mathrm{~ns}$ Trp lifetime reflects the conformation of DREAM associated with lipid vesicles or membranes.

\subsubsection{DREAM stability increases in the presence of $\mathrm{Ca}^{2+} / \mathrm{Mg}^{2+}$}

Association of $\mathrm{Mg}^{2+}$ and/or $\mathrm{Ca}^{2+}$ to DREAM EF-hands increases the stability of the protein, although to different extent. The unfolding data (Figure 4.7b) show that DREAM unfolds through a partially unfolded intermediate state that is populated at $\sim 4.5 \mathrm{M} \mathrm{GuHCl}$ in the case of Apo and $\mathrm{Mg}^{2+}$ DREAM and at $~ 5.0 \mathrm{M} \mathrm{GuHCl}$ for $\mathrm{Ca}^{2+}$ and $\mathrm{Ca}^{2+} \mathrm{Mg}^{2+} \mathrm{DREAM}$. Further increase in the $\mathrm{GuHCl}$ concentration promotes protein 
destabilization towards the unfolded state. Unlike small, single domain proteins that fold with two-state kinetics without populating intermediate state, multi-domain proteins commonly exhibit unfolding intermediates. Furthermore, protein stability and folding is modified by the presence of higher order clusters of aromatic residues (Burley et al., 1985; Song et al., 2013). Such residues are usually highly conserved and their substitution often leads to the destabilization of protein structure. I have identified two aromatic hydrophobic cores in the DREAM structure (Figure 4.1) that i) are formed by residues conserved among NCS members, ii) have a relative solvent accessibility smaller than $10 \%$ (Rost and Sander, 1994), and iii) groups side chains that are within a distance of $4.5 \AA$ of each other (Arunachalam and Gautham 2008). One hydrophobic core is formed by an aromatic tetramer of F171, Y174, F218, and F235 and is found at the C-terminal domain (Figure 4.1). All these residues are in a helical structural motif. The cluster formation brings together $\alpha$-helices 6,8 , and 9 , which may stabilize the C-terminal domain. The second hydrophobic core was found within the N-terminal domain and is a trimer of phenylalanine residues (F114, F135, and F138) that are found in $\alpha$-helices 3 and 4. Analogously to the C terminal domain, the interactions between these residues increase the stability of the Nterminal domain. In addition, W169 from the C-terminal domain interacts with F138 from the N-terminal domain and such inter-domain interactions may contribute to DREAM stability.

Considering that the $\mathrm{Ca}^{2+}$ association to EF-hand 3 and EF-hand 4 increases the stability of the partially unfolded intermediate with respect to the unfolded protein, I propose that $\alpha$-helices 6,8 , and 9 form a stable hydrophobic core that unfolds during the intermediate between the transition and unfolded states. The N-terminal domain that lacks 
more extended hydrophobic core(s) may be less stable than the $\mathrm{C}$-terminal domain and thus may be destabilized during the initial unfolding transition. This is further supported by the fact that the stability of the $\mathrm{N}$-terminal domain is increased by the $\mathrm{Mg}^{2+}$ association to EFhand 2, which is located in the N-terminal domain. Thus the unfolding data suggest that the $\mathrm{N}$-terminal domain unfolds during the first transition whereas the $\mathrm{C}$-terminal domain is destabilized at increased denaturant concentration. An analogous unfolding mechanism was reported previously for another member of the NCS family, neuronal calcium sensor 1 (NCS-1) (Muralidhar et al., 2005; Aravind et al., 2008; Heidarsson et al. 2013). That study reported that the folding mechanism and protein stability of NCS-1 are altered by the presence of $\mathrm{Ca}^{2+}$ and $\mathrm{Mg}^{2+}$ ions as well as by the protein myristoylation at the $\mathrm{N}$-terminus. Non-myristoylated NCS1 unfolds through a three step mechanism with an overall stability of $\Delta \mathrm{G}_{\mathrm{N} \rightarrow \mathrm{U}}=12.9 \pm 0.7 \mathrm{kcal} \mathrm{mol}^{-1}$ for ApoNCS1, and $\Delta \mathrm{G}_{\mathrm{N} \rightarrow \mathrm{U}}=14.5 \pm 1.6 \mathrm{kcal} \mathrm{mol}^{-1}$ for holo-NCS1. These values are comparable to those determined here for Apo and $\mathrm{Ca}^{2+}$ bound DREAM. These results indicate a similar folding mechanism for NCS1 and DREAM, and possibly other members of the NCS family. This is further supported by a kinetic study of folding mechanisms using optical tweezers (Heidarsson et al., 2013). The authors have reported that the folding of the $\mathrm{C}$-terminal domain precedes the folding of the $\mathrm{N}$-terminal domain, and that the C-terminal domain is stabilized by aromatic interactions between $\alpha$ helices 6, 8, and 9 .

\subsubsection{Summary}

Despite the low impact of $\mathrm{Mg}^{2+}$ association with EF-hand 2 on the DREAM tertiary structure, the presence of $\mathrm{Mg}^{2+}$ stabilizes the native state with respect to a partially unfolded state. The W169 lifetime data indicates that DREAM can adopt an additional conformation 
with a long W169 lifetime of $7.5 \mathrm{~ns}$ that may reflect conformations of DREAM populated in the presence of lipid membranes and vesicles. The results show that DREAM unfolding is a complex process that occurs through a partially unfolded, intermediate state. $\mathrm{The}^{2{ }^{2+}}$ association to EF-hand 3 and 4 stabilizes the partially unfolded state with respect to the unfolded state, whereas the association of $\mathrm{Mg}^{2+}$ increases the stability of the native state only in the presence of $\mathrm{Ca}^{2+}$. These results suggest that the intracellular fluctuations in $\mathrm{Ca}^{2+}$ and $\mathrm{Mg}^{2+}$ concentrations control the global conformation and stability of DREAM and likely regulate its interactions with intracellular partners. 


\section{CALCIUM AND MAGNESIUM BINDING TO THE C-TERMINAL DOMAIN MODULATES DREAM STRUCTURE, DYNAMICS, AND STABILITY}

(The results in this chapter were described in a manuscript that will be submitted by Pham, Gonzalez, and Miksovska)

\subsection{Background and significance}

Downstream regulatory element antagonist modulator (DREAM), also known as calsenilin or KChIP3, is a neuronal calcium sensor (NCS) involved in regulation of important neuronal processes including pain modulation, $A \beta$-amyloid production, and potassium channel activity (Buxbaum et al., 1998; Carrion et al., 1999; An et al., 2000). The intracellular function of DREAM depends on its intracellular localization and interactions with target proteins. $\mathrm{Ca}^{2+}$ free DREAM forms a tetramer that binds to the downstream regulatory element (DRE) sequence of prodynorphin and c-fos genes to repress transcription of these genes. Upon binding to $\mathrm{Ca}^{2+}$, DREAM tetramer dissociates into a stable dimer with a decreased affinity toward DRE (Carrion et al., 1999; Ledo et al., 2000; Osawa et al., 2001, Osawa et al., 2005). In the cytoplasm, DREAM interacts with the $\mathrm{C}$ terminal portion of Alzheimer's disease associated proteins, presenilin 1 and presenilin 2, and elevates the formation of A $\beta$-amyloid peptides (Buxbaum et al., 1998). In vivo studies have shown that DREAM overexpression is associated with an increased formation of A $\beta$-amyloid peptide and concomitant cell death (Jo et al. 2001; Jo et al., 2003; Jo et al., 2004). In addition, the level of $A \beta$ peptide was significantly reduced in the cerebellum and in the cortex of DREAM knock-out mice (Lilliehook et al., 2003). DREAM also interacts with A-type voltage-gated potassium channels (Kv4) and modulates both the kinetic properties and cell surface accumulation of Kv4 channels (An et al., 2000). 
Recently, it has been proposed that $\mathrm{Ca}^{2+}$ DREAM forms a complex with calmodulin, whereas ApoDREAM binds to a regulatory subunit of calcineurin (Ramachandran et al., 2012). The interactions with multiple neuronal proteins highlight a wide range of DREAM neuronal functions under physiological and pathological conditions.

Unlike traditional EF-hand calcium sensors, such as calmodulin and troponin C, that carry four EF-hands with a high affinity for $\mathrm{Ca}^{2+}$, members of the NCS family carry only 2 or 3 functional EF-hands that bind $\mathrm{Ca}^{2+}$ with a high affinity. In DREAM, EF-hand 1 does not bind to $\mathrm{Mg}^{2+}$ or $\mathrm{Ca}^{2+}$ ion due to the presence of conserved residues $\mathrm{C} 104$ and P105 on the first $\mathrm{Ca}^{2+}$ binding loop, which abolishes the affinity for $\mathrm{Mg}^{2+}$ and $\mathrm{Ca}^{2+}$ (Osawa et al., 2005; Lusin et al., 2008). The presence of Asp (D150) at the $12^{\text {th }}$ position of the EFhand 2 binding loop promotes specific binding to $\mathrm{Mg}^{2+}$ with relatively high affinity $\left(\mathrm{K}_{\mathrm{d}}=\right.$ $13 \mu \mathrm{M}$ ), whereas EF-hands 3 and 4 functionally bind to $\mathrm{Ca}^{2+}\left(\mathrm{K}_{\mathrm{d}} \sim 1-10 \mu \mathrm{M}\right)$ (Osawa et al., 2005; Yu et al., 2007; Lusin et al., 2008). Association of $\mathrm{Ca}^{2+}$ with DREAM triggers protein dimerization that inhibits the interaction with DNA (Lusin et al., 2008). The 3dimensional structures of DREAM and DREAM-C reveal that the C-terminal $\alpha$-helix (residues 243-254) and the residues from EF-hand 3 (L161, A170, M191, and L194) and EF-hand 4 (F218, M222, and C239) form a large hydrophobic cavity (Lusin et al., 2008) on at the C-terminal domain that provides a binding interface for the site 1 of the $\mathrm{K}_{\mathrm{v}} 4.3$ channels as well as for small hydrophobic molecules (Gonzalez and Miksovska 2014, Gonzalez et al., 2014). In addition, a hydrophobic cleft located between the flexible loop connecting EF-hand 3 and EF-hand 4 (residues 198 - 211) and the C-terminal $\alpha$-helix was thought as a binding site for interaction with target proteins during calcium signaling ( $\mathrm{Yu}$ et al., 2007). 
However, the structure of Apo and $\mathrm{Mg}^{2+}$ bound DREAM are currently unknown that prevents us from a full understanding of conformational transition associated with $\mathrm{Ca}^{2+}$ or $\mathrm{Mg}^{2+}$ binding to this neuronal regulator and the role of the $\mathrm{Ca}^{2+} / \mathrm{Mg}^{2+}$ in DREAM interactions with intracellular partners. Despite considerable efforts to understand the mechanism of $\mathrm{Ca}^{2+}$ signal transduction within DREAM protein, little information is known about the dynamics of $\mathrm{Ca}^{2+}$ induced conformational changes in the full length protein as well as in the C-terminal domain. In addition, the impact of $\mathrm{Ca}^{2+}$ and/or $\mathrm{Mg}^{2+}$ association on DREAM-C (residues 161-256) structure and its stability has not been fully established.

In this study I have characterized the conformational transition induced by $\mathrm{Ca}^{2+}$ or $\mathrm{Mg}^{2+}$ binding to DREAM-C by monitoring the fluorescence properties of an intrinsic tryptophan residue (W169). In addition, extrinsic fluorescent probes: 8-anilino-1naphthalenesulfonic acid ammonium salt (1,8-ANS) and 9-diethylamino-5Hbenzo[alpha]phenoxazine-5-one (nile red) were employed to monitor the hydrophobic properties on DREAM-C surface and the oligomerization state of DREAM-C as a function of $\mathrm{Ca}^{2+}$ or $\mathrm{Mg}^{2+}$. The thermodynamic parameters for $\mathrm{Ca}^{2+}$ or $\mathrm{Mg}^{2+}$ binding to DREAM-C were probed using isothermal titration calorimetry (ITC), and the effects of $\mathrm{Ca}^{2+} / \mathrm{Mg}^{2+}$ binding on DREAM-C stability were investigated in protein unfolding experiments.

\subsection{Results}

\subsubsection{Thermodynamics of $\mathrm{Ca}^{2+}$ and/or $\mathrm{Mg}^{2+}$ binding to DREAM-C}

The isotherm for $\mathrm{Ca}^{2+}$ association to ApoDREAM-C is shown in Figure 5.1 and the thermodynamic parameters are summarized in Table 5.1. 


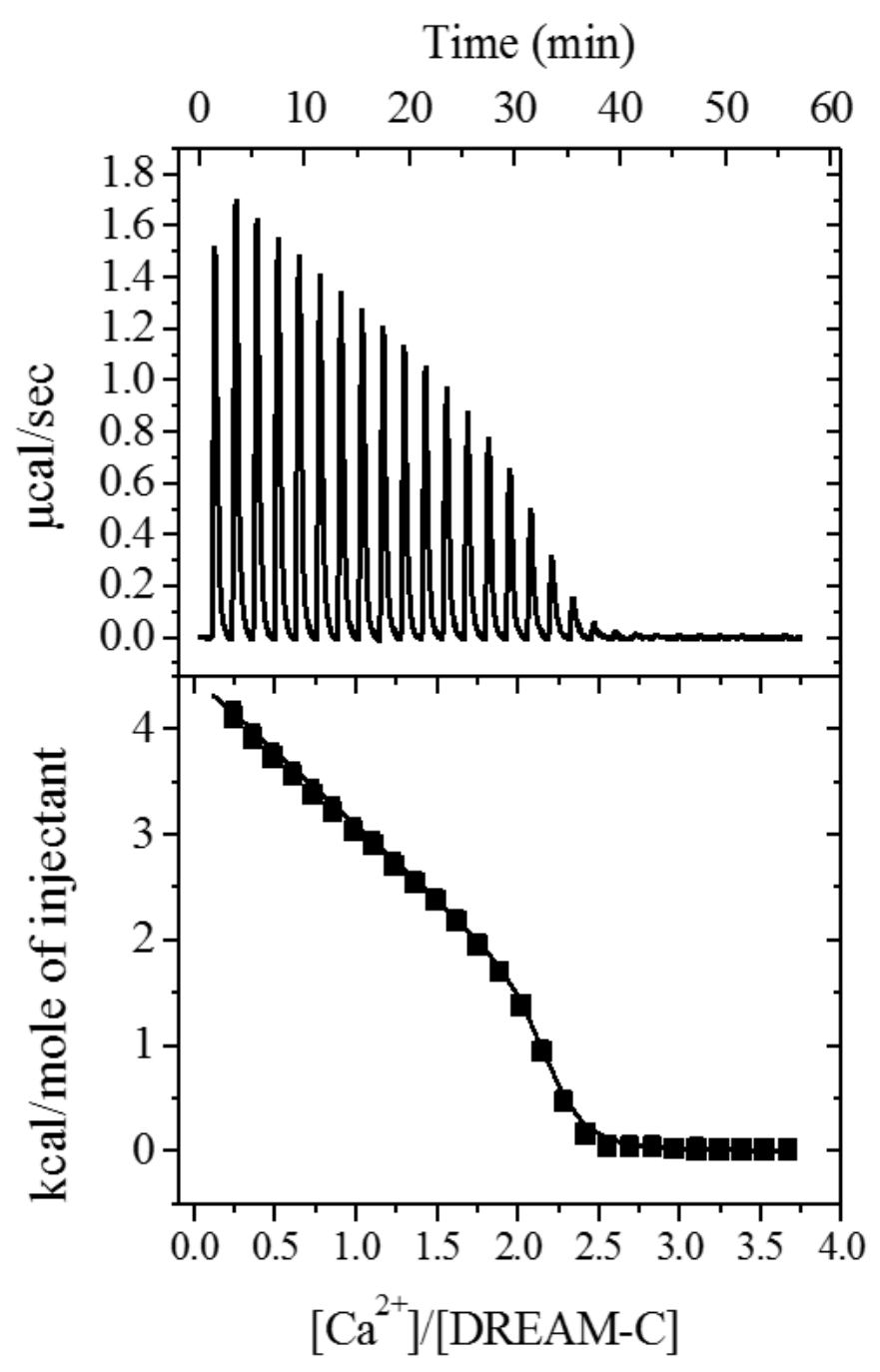

Figure 5.1: Binding isotherm for $\mathrm{Ca}^{2+}$ association to DREAM-C in the absence of $\mathrm{Mg}^{2+}$. The top panel represents a raw power titration curve, the bottom panel shows integration data, and the solid line represents the two sets of sites fitting curve.

Association of $\mathrm{Ca}^{2+}$ at EF-hands 3 and 4 of DREAM-C endothermically $\left(\Delta \mathrm{H}_{1}=5.5\right.$ $\mathrm{kcal} \mathrm{mol}^{-1}$ and $\left.\Delta \mathrm{H}_{2}=1.1 \mathrm{kcal} \mathrm{mol}^{-1}\right)$ with a sub-micromolar affinity $\left(\mathrm{K}_{\mathrm{d} 1}=0.3 \pm 0.1 \mu \mathrm{M}\right.$ and $\left.\mathrm{K}_{\mathrm{d} 2}=0.9 \pm 0.2 \mu \mathrm{M}\right)$, respectively. The positive enthalpy change is consistent with the high dehydration energy of the divalent $\mathrm{Ca}^{2+}$ ions upon binding to DREAM-C (Osawa et al., 2005). The binding isotherm for $\mathrm{Ca}^{2+}$ association to $\mathrm{Mg}^{2+} \mathrm{DREAM}-\mathrm{C}$ resulted in approximately a two-fold larger equilibrium dissociation constant for one binding site $\left(\mathrm{K}_{\mathrm{d} 1}\right.$ 
$=0.6 \pm 0.1 \mu \mathrm{M}$ and $\left.\mathrm{K}_{\mathrm{d} 2}=1.0 \pm 0.3 \mu \mathrm{M}\right)$ with the $\mathrm{Ca}^{2+}$ binding to one site being endothermic $\left(\Delta \mathrm{H}_{1}=9.4 \pm 4.0 \mathrm{kcal} \mathrm{mol}^{-1}\right)$ and to the other site being exothermic $\left(\Delta \mathrm{H}_{2}=-15.0 \pm 1.4 \mathrm{kcal}\right.$ $\left.\mathrm{mol}^{-1}\right)$. The analysis of the binding isotherm for $\mathrm{Mg}^{2+}$ association to ApoDREAM reveals the presence of two binding sites, with one site being a high affinity site $\left(K_{d 1}=180 \pm 10\right.$ $\mu \mathrm{M}$ and $\left.\Delta \mathrm{H}_{1}=1.5 \pm 0.1 \mathrm{kcal} \mathrm{mol}^{-1}\right)$ and another site with a milimolar affinity $\left(\mathrm{K}_{\mathrm{d} 2}=1.7 \pm\right.$ $0.1 \mathrm{mM}$ and $\left.\Delta \mathrm{H}_{2}=3.5 \pm 0.1 \mathrm{kcal} \mathrm{mol}{ }^{-1}\right)$. The endothermic $\mathrm{Mg}^{2+}$ association to ApoDREAM-C points out towards the absence of pronounced structural changes associated with $\mathrm{Mg}^{2+}$ binding to DREAM-C.

Table 5.1: Thermodynamic parameters for $\mathrm{Ca}^{2+}$ and/or $\mathrm{Mg}^{2+}$ binding to DREAM-C

\begin{tabular}{|l|c|c|c|c|c|c|c|c|}
\hline DREAM-C & $n_{1}$ & $\begin{array}{c}\mathrm{K}_{\mathrm{d} 1} \\
(\mu M)\end{array}$ & $\begin{array}{c}\Delta \mathrm{H}_{1} \\
(\mathrm{kcal} / \mathrm{mol})\end{array}$ & $\begin{array}{c}\Delta \mathrm{S}_{1} \\
(\mathrm{cal} / \mathrm{mol} \mathrm{K})\end{array}$ & $n_{2}$ & $\mathrm{~K}_{\mathrm{d} 2}$ & $\Delta \mathrm{H}_{2}$ & $\Delta \mathrm{S}_{2}$ \\
\hline $\begin{array}{l}\mathrm{Ca}^{2+} \text { versus } \\
\text { Apo }\end{array}$ & $0.9 \pm 0.1$ & $0.3 \pm 0.1$ & $5.5 \pm 0.8$ & 49 & $1.2 \pm 0.1$ & $0.9 \pm 0.2$ & $1.1 \pm 0.7$ & 31 \\
\hline $\begin{array}{l}\mathrm{Ca}^{2+} \text { versus } \\
\mathrm{Mg}^{2+} \text { bound }\end{array}$ & $1.3 \pm 0.2$ & $0.6 \pm 0.1$ & $9.4 \pm 4.0$ & 60 & $0.7 \pm 0.2$ & $1.0 \pm 0.3$ & $-15.0 \pm 1$ & -24 \\
\hline $\begin{array}{l}\mathrm{Mg}^{2+} \text { versus } \\
\text { Apo }\end{array}$ & 1.0 & $180 \pm 10$ & $1.5 \pm 0.1$ & 22 & 0.97 & $\begin{array}{l}1.7 \times 10^{3} \pm \\
0.1 \times 10^{3}\end{array}$ & $3.5 \pm 0.1$ & 25 \\
\hline
\end{tabular}

\subsubsection{Secondary structural changes induced by $\mathrm{Mg}^{2+}$ and/or $\mathrm{Ca}^{2+}$ binding}

Changes in the secondary structure of DREAM-C upon $\mathrm{Ca}^{2+}$ and/or $\mathrm{Mg}^{2+}$ biding were monitored by near-UV circular dichroism (Figure 5.2). A small, but reproducible decrease in the $\mathrm{CD}$ signal was observed at $220 \mathrm{~nm}$ upon addition of $\mathrm{Mg}^{2+}$ to ApoDREAM$\mathrm{C}$, and an additional increase in the $220 \mathrm{~nm}$ signal was recorded upon $\mathrm{Ca}^{2+}$ binding to $\mathrm{Mg}^{2+}$ DREAM, indicating that association of both divalent ions leads to an increase in the 
protein $\alpha$-helical structure. The CD signal for the $\mathrm{Ca}^{2+}$ DREAM-C in the absence of $\mathrm{Mg}^{2+}$ is identical to that measured in the presence of $\mathrm{Mg}^{2+}$, highlighting that $\mathrm{Ca}^{2+}$ association to $\mathrm{Mg}^{2+}$ DREAM-C cancels out structural changes triggered by $\mathrm{Mg}^{2+}$ binding. The increase in the $\alpha$-helical structure upon $\mathrm{Ca}^{2+}$ binding to EF-hands is common among the calcium binding proteins and has been observed previously for other EF-hand proteins (Burgoyne and Weiss, 2001).

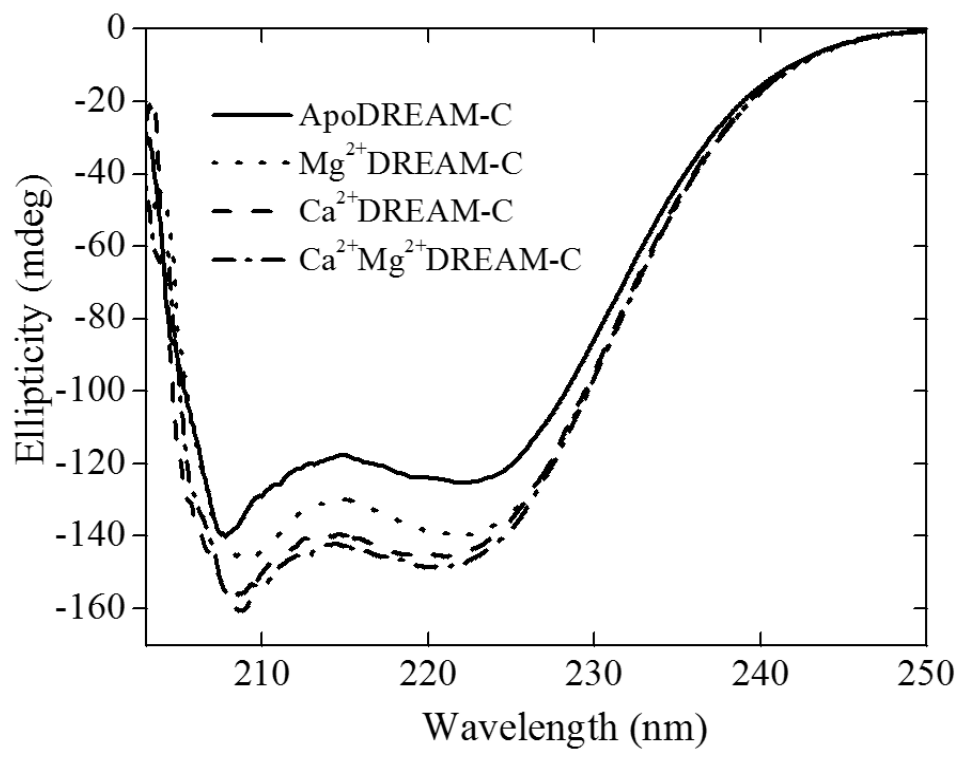

Figure 5.2: The CD spectra of DREAM-C in the presence/absence of $\mathrm{Ca}^{2+}$ and/or $\mathrm{Mg}^{2+}$

\subsubsection{Tertiary structural changes triggered by $\mathrm{Mg}^{2+}$ binding differ from those associated with $\mathrm{Ca}^{2+}$ binding}

The single tryptophan residue in DREAM-C (W169) provides an excellent probe to monitor changes in tertiary structure induced by the binding of $\mathrm{Mg}^{2+}$ and/or $\mathrm{Ca}^{2+}$ to DREAM-C. The W169 emission spectrum of ApoDREAM-C is broad with a maximum at $349 \mathrm{~nm}$ (Figure 5.3) and the emission intensity of DREAM-C increases, without a concomitant shift in the maximum wavelength, upon addition of $\mathrm{Mg}^{2+}$, indicating that in 
the W169 quenching is reduced in the $\mathrm{Mg}^{2+}$ bound form. The emission maximum at 349 $\mathrm{nm}$ observed for Apo and $\mathrm{Mg}^{2+}$ DREAM-C is similar to that of tryptophan in aqueous solution $(\sim 350 \mathrm{~nm})$, pointing out that the W169 side chain is located near a polar environment. Addition of $\mathrm{Ca}^{2+}$ to Apo or $\mathrm{Mg}^{2+}$ DREAM leads to a decrease in $\mathrm{W} 169$ emission intensity and a concomitant hypsochromic shift to $344 \mathrm{~nm}$ due to the transfer of the W169 side chain into a less polar surrounding. Analogously, as observed in CD measurements, the identical emission spectra of the $\mathrm{Ca}^{2+}$ and $\mathrm{Ca}^{2+} \mathrm{Mg}^{2+}$ bound form of DREAM-C suggest that $\mathrm{Ca}^{2+}$ binding to EF-hands cancels the structural changes induced by $\mathrm{Mg}^{2+}$ association to DREAM.

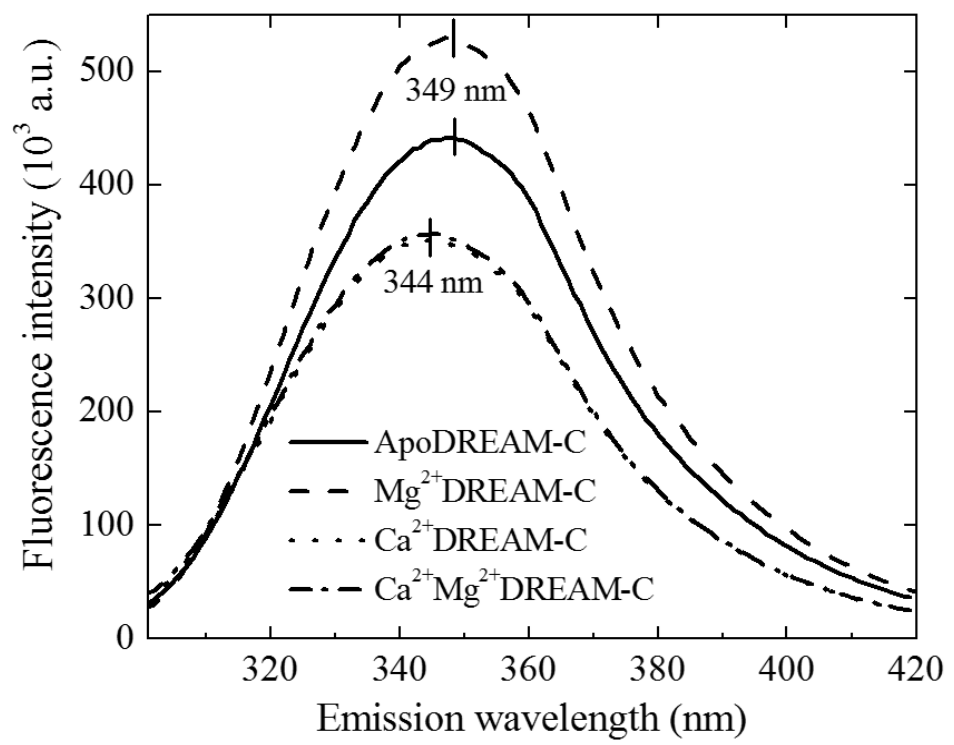

Figure 5.3: The fluorescence steady-state emission spectra of intrinsic W169 residue in DREAM-C.

The W169 emission quenching by acrylamide was used to further investigate the solvent accessibility of W169 site chain in ligand free and ligand bound DREAM-C and thus reflects ligand induced alteration of the tertiary structure and/or and conformational 
dynamics (Augusteyn et al., 1988). The Stern-Volmer plots are shown in Figure 5.4 and the Stern-Volmer constants are shown in Table 5.2.

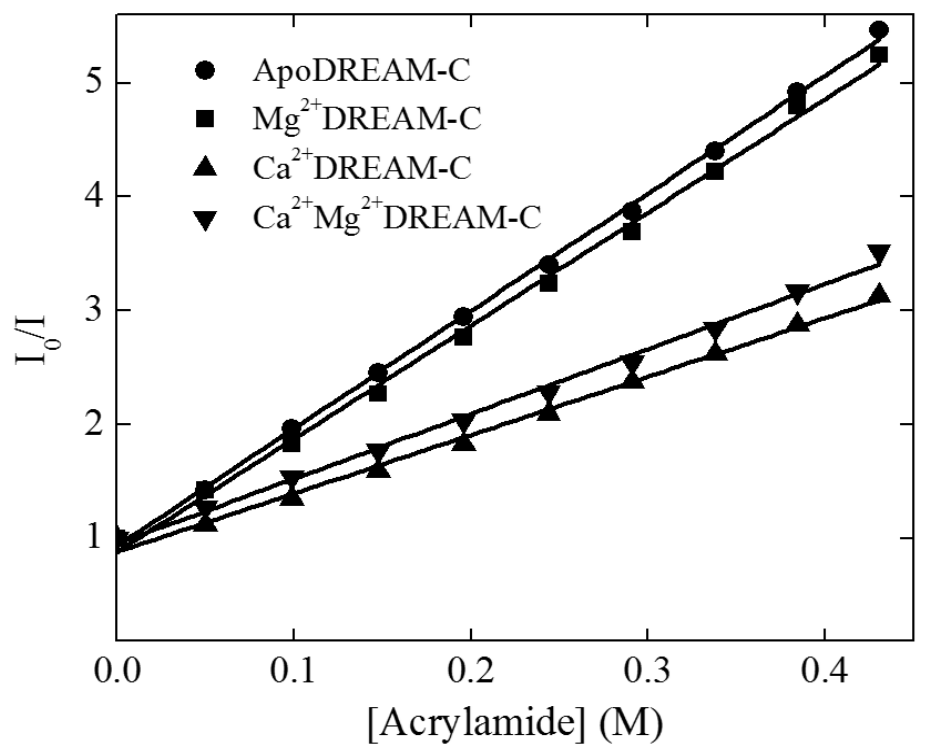

Figure 5.4: Stern-Volmer plot for acrylamide quenching of DREAM-C in the absence/presence of $\mathrm{Ca}^{2+}$ and $\mathrm{Mg}^{2+}$.

Table 5.2: The Stern-Volmer constants $\left(\mathrm{K}_{\mathrm{sv}}\right)$ for acrylamide quenching of DREAM-C

\begin{tabular}{|c|c|l|c|}
\hline Protein & $K_{S V}\left(M^{-1}\right)$ & \multicolumn{1}{|c|}{ Protein } & $K_{S V}\left(M^{-1}\right)$ \\
\hline ApoDREAM-C & $10.4 \pm 0.1$ & $\mathrm{Ca}^{2+}$ DREAM-C & $5.1 \pm 0.1$ \\
\hline $\mathrm{Mg}^{2+}$ DREAM-C & $9.9 \pm 0.2$ & $\mathrm{Ca}^{2+} \mathrm{Mg}^{2+}$ DREAM-C & $5.7 \pm 0.1$ \\
\hline
\end{tabular}

The increased $\mathrm{K}_{\mathrm{Sv}}$ values for the ApoDREAM-C and $\mathrm{Mg}^{2+}$ DREAM-C $\left(\mathrm{K}_{\mathrm{sv}}=10.4\right.$ $\pm 0.1 \mathrm{M}^{-1}$ and $9.9 \pm 0.2 \mathrm{M}^{-1}$, respectively) suggest that the W169 indole ring is nearly fully exposed to the solvent, in agreement with the steady-state emission data. Association of $\mathrm{Ca}^{2+}$ to Apo or $\mathrm{Mg}^{2+}$ DREAM-C induces a conformational change that significantly reduces the solvent accessibility of the $\mathrm{W} 169$ residue as evident from the $\mathrm{K}_{\mathrm{sv}}$ values of 5.1 $\pm 0.1 \mathrm{M}^{-1}$ and $5.7 \pm 0.1 \mathrm{M}^{-1}$, respectively. 
The frequency-domain intensity decay data for DREAM-C are shown in Figure 5.5. The data were analyzed using a Gaussian-Discrete double decay model and the recovered parameters are shown in Table 5.3.

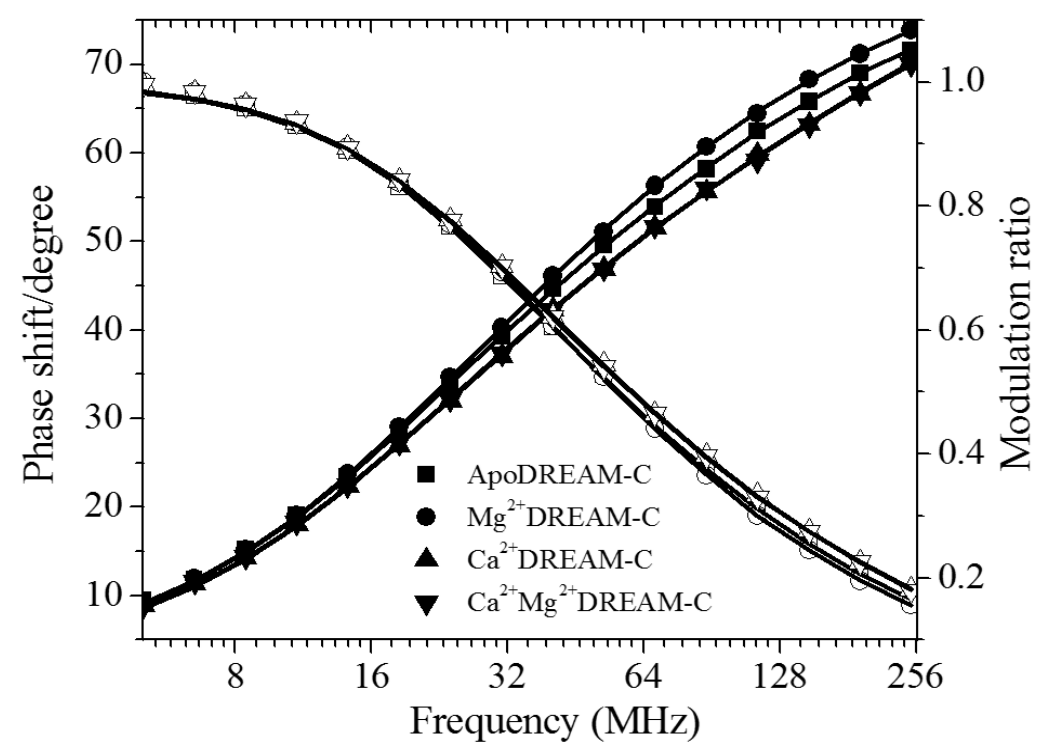

Figure 5.5: Frequency-domain intensity decays for W169 in the presence/absence of $\mathrm{Ca}^{2+}$ and/or $\mathrm{Mg}^{2+}$. Phase delays are solid symbols, modulation ratios are empty symbols, and the solid lines represent the calculated fitting curves.

The discrete component of the emission decay has a long lifetime of $\sim 7.3 \mathrm{~ns}$ that is independent of the presence of $\mathrm{Mg}^{2+}$ or $\mathrm{Ca}^{2+}$. The continuous Gaussian distribution model has a center value of distribution of $3.0 \mathrm{~ns}$ for ApoDREAM and $3.5 \mathrm{~ns}$ for $\mathrm{Mg}^{2+}$ DREAM-C, and decreases to $2.5 \mathrm{~ns}$ in the presence of $\mathrm{Ca}^{2+}$. The Gaussian distribution width decreases in the presence of $\mathrm{Ca}^{2+}$ from $1.6 \mathrm{~ns}$ as observed for ApoDREAM-C to 1.3 ns determined, for $\mathrm{Ca}^{2+}$ DREAM-C. 
Table 5.3: Fluorescence decay parameters for DREAM-C intrinsic tryptophan residue. Data were analyzed using a Gaussian-discrete model

\begin{tabular}{|c|c|c|c|c|c|c|}
\hline Protein & $\begin{array}{c}\tau_{1} \\
(\mathrm{~ns})\end{array}$ & $\begin{array}{c}w_{1} \\
(\mathrm{~ns})\end{array}$ & $f_{1}$ & $\begin{array}{c}\tau_{2} \\
(\mathrm{~ns})\end{array}$ & $f_{2}$ & $\chi^{2}$ \\
\hline ApoDREAM-C & 3.0 & 1.6 & 0.52 & 7.4 & 0.48 & 0.9 \\
\hline $\mathrm{Mg}^{2+} \mathrm{DREAM}-\mathrm{C}$ & 3.5 & 1.7 & 0.56 & 7.3 & 0.44 & 1.4 \\
\hline $\mathrm{Ca}^{2+}$ DREAM-C & 2.5 & 1.3 & 0.49 & 7.2 & 0.51 & 1.3 \\
\hline $\mathrm{Ca}^{2+} \mathrm{Mg}^{2+} \mathrm{DREAM}-\mathrm{C}$ & 2.5 & 1.3 & 0.49 & 7.2 & 0.51 & 1.4 \\
\hline \multicolumn{7}{|c|}{$\begin{array}{l}\tau_{1} \text { is the center of Gaussian distribution, } f \text { is fractional contribution, } w \text { is the Gaussian } \\
\text { distribution width, and } \tau_{2} \text { is lifetime of the discrete component. The constant } \\
\text { standard error of } 0.2^{\circ} \text { for the phase angle and } 0.004 \text { for modulation were used for } \\
\text { data analysis }\end{array}$} \\
\hline
\end{tabular}

\subsubsection{Binding of $\mathrm{Ca}^{2+}$ and/or $\mathrm{Mg}^{2+}$ triggers distinct changes in hydrophobic surface of DREAM-C protein}

The structural transition induced by $\mathrm{Ca}^{2+}$ and/or $\mathrm{Mg}^{2+}$ binding to DREAM-C in terms of changes in the hydrophobic surface was probed using the hydrophobic fluorescence probes 1,8-ANS and nile red. The steady-state emission spectra demonstrate that both probes bind to DREAM. However, unlike 1,8-ANS emission, the emission of nile red is sensitive to the structural changes triggered by $\mathrm{Mg}^{2+}$ association to DREAM-C (Figure 5.6, panels A and B).

Nile red in $20 \mathrm{mM}$ Tris buffer exhibits weak emission intensity with a maximum at $657 \mathrm{~nm}$. In the presence of ApoDREAM-C, the emission intensity increases approximately 2 times and the maximum shifts to $642 \mathrm{~nm}$. A further increase in the emission intensity and concomitant bathochromic shift to $628 \mathrm{~nm}$ were observed for nile red: $\mathrm{Mg}^{2+}$ DREAM-C complex. 

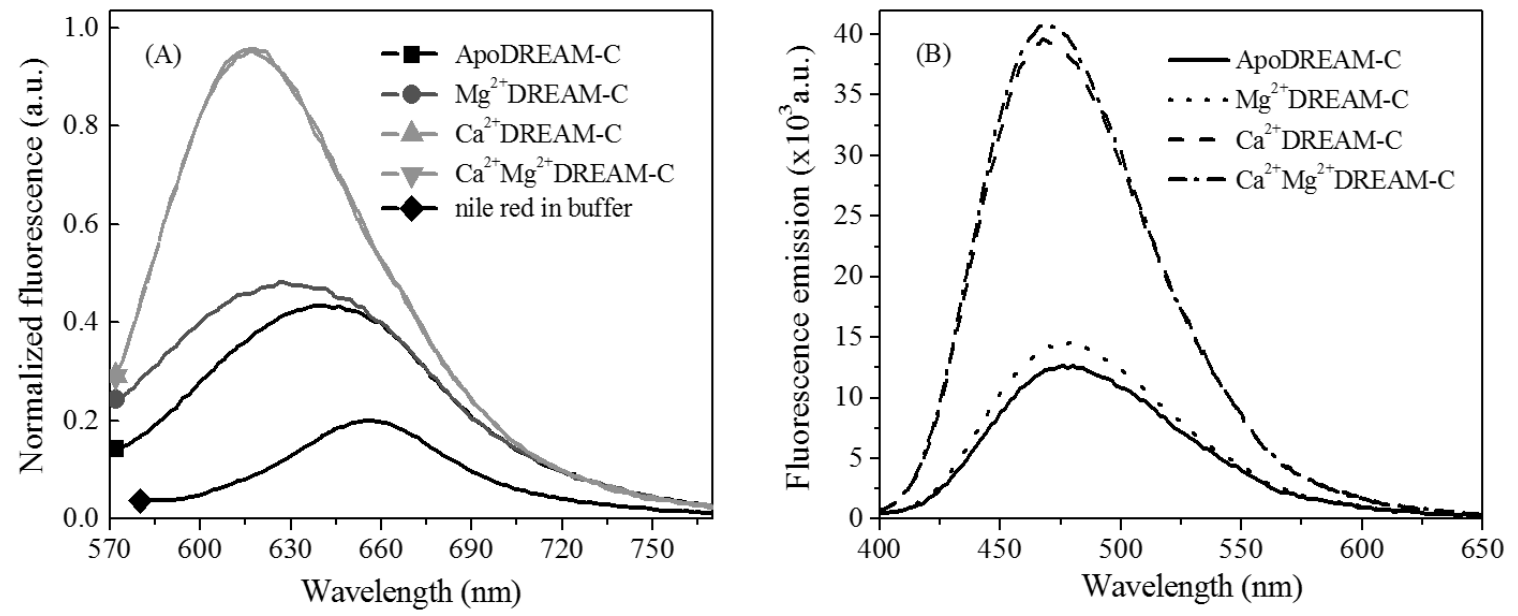

Figure 5.6: Fluorescence steady-state emission spectra of nile red (A) and 1,8-ANS (B) binding to DREAM-C in the presence or absence of $\mathrm{Ca}^{2+}$ and/or $\mathrm{Mg}^{2+}$.

In the presence of $\mathrm{Ca}^{2+} \mathrm{DREAM}$ and $\mathrm{Ca}^{2+} \mathrm{Mg}^{2+} \mathrm{DREAM}-\mathrm{C}$, the emission intensity of nile red increases approximately 5 times and exhibits a maximum at $616 \mathrm{~nm}$. These data are consistent with $\mathrm{Mg}^{2+}$ and $\mathrm{Ca}^{2+}$ triggered structural changes that result in a more hydrophobic binding site for nile red. The increase in the emission intensity of nile red: $\mathrm{Mg}^{2+} \mathrm{DREAM}-\mathrm{C}$ and nile red:Ca ${ }^{2+} \mathrm{DREAM}-\mathrm{C}$ complexes is consistent with the observed equilibrium dissociation constants (Table 5.4). Overall, the nile red binding to DREAM-C is relatively weak, $\left(\mathrm{K}_{\mathrm{d}}=122 \pm 53 \mu \mathrm{M}\right)$ and increases in the presence of $\mathrm{Mg}^{2+}$ and $\mathrm{Ca}^{2+}, \mathrm{K}_{\mathrm{d}}=50 \pm 5 \mu \mathrm{M}$ and $10 \pm 1 \mu \mathrm{M}$, respectively.

Table 5.4: Fluorescence decay parameters for nile red:DREAM-C. The experimental data were analyzed using a discrete component and continuous Gaussian distribution model.

\begin{tabular}{|l|c|c|c|c|c|c|c|}
\hline DREAM-C & $\mathrm{K}_{\mathrm{d}}(\mu \mathrm{M})$ & $\tau_{1}(\mathrm{~ns})$ & $f_{1}$ & $\tau_{2}(\mathrm{~ns})$ & $f_{2}$ & $\mathrm{w}$ & $\chi^{2}$ \\
\hline Apo & $122 \pm 53$ & 0.23 & 0.17 & $3.4 \pm 0.3$ & 0.83 & 1.6 & 0.86 \\
\hline $\mathrm{Mg}^{2+}$ & $50 \pm 5$ & 0.23 & 0.09 & $4.1 \pm 0.4$ & 0.91 & 1.3 & 0.85 \\
\hline $\mathrm{Ca}^{2+}$ & $10 \pm 1$ & 0.23 & 0.05 & $3.5 \pm 0.1$ & 0.95 & 1.7 & 0.69 \\
\hline $\mathrm{Ca}^{2+} \mathrm{Mg}^{2+}$ & $12 \pm 2$ & 0.23 & 0.06 & $3.4 \pm 0.2$ & 0.94 & 1.7 & 0.77 \\
\hline
\end{tabular}

The lifetime of the discrete component was set as a linked variable. Phase and modulation errors were set at $\leq 0.2^{0}$ and $\leq 0.002$ respectively. 


\subsubsection{Contribution of $\mathrm{Mg}^{2+}$ and $\mathrm{Ca}^{2+}$ in the stability of DREAM-C}

In order to investigate the impact of $\mathrm{Ca}^{2+} / \mathrm{Mg}^{2+}$ on DREAM-C stability, $\mathrm{GuHCl}$ induced unfolding of DREAM-C was monitored using circular dichroism. The unfolding profiles (Figure 5.7) were then analyzed using equation 3.61 in section 3.2.4.3 for a twostate unfolding model. The thermodynamic parameters are summarized in Table 5.5.

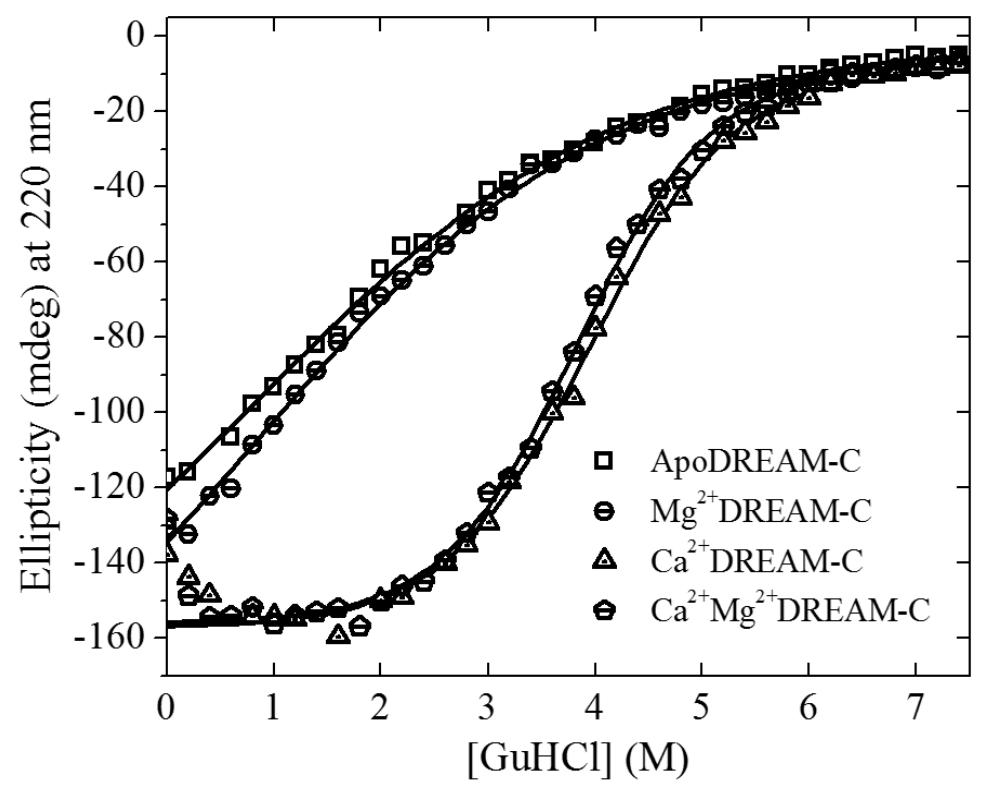

Figure 5.7: $\mathrm{GuHCl}$ induced unfolding DREAM-C in the presence or absence of $\mathrm{Ca}^{2+}$ and/or $\mathrm{Mg}^{2+}$. Unfolding parameters were recovered using equation 3.61 in section 3.2.4.3 for a two-state unfolding model.

The stability of Apo and $\mathrm{Mg}^{2+}$ DREAM-C is low with $\Delta \mathrm{G} \sim 0.3 \mathrm{kcal} \mathrm{mol}^{-1}$ suggesting that the DREAM-C is partially destabilized under native like conditions. Both unfolding curves have a non-sigmoidal profile that can be associated with a noncooperative protein unfolding. On the other hand, the unfolding profiles of $\mathrm{Ca}^{2+}$ DREAM$\mathrm{C}$ in the absence and presence of $5 \mathrm{mM} \mathrm{Mg}{ }^{2+}$ has a midpoint at $\sim 4 \mathrm{M} \mathrm{GuHCl}$ and are characterized with sigmoidal unfolding curves indicative of a cooperative unfolding 
transition. In addition, a small increase in the $\alpha$-helical structure at sub-denaturant concentration ( 0 to $1 \mathrm{M}$ ) was observed for $\mathrm{Ca}^{2+}$ bound DREAM-C. Analogous increase in the negative ellipticity was reported for $\mathrm{Ca}^{2+}$ bound form of the non-myristoylated neuronal calcium sensor 1 and were associated with the binding of denaturant molecules and concomitant changes in dihedral angles. The $\mathrm{Ca}^{2+}$ association to EF-hands 3 and 4 increases the protein stability by $\sim 3.0 \mathrm{kcal} \mathrm{mol}^{-1}$ compared to the Apo protein.

Table 5.5: Thermodynamic parameters for DREAM-C unfolding

\begin{tabular}{|l|c|c|c|}
\hline \multicolumn{1}{|c|}{ Protein } & $\begin{array}{c}\Delta \mathrm{G}_{\mathrm{U}} \\
\left(\mathrm{kcal} \mathrm{mol}^{-1}\right)\end{array}$ & $\begin{array}{c}\mathrm{m}_{\mathrm{U}} \\
\left(\mathrm{kcal} \mathrm{mol}^{-1} \mathrm{M}^{-1}\right)\end{array}$ & $\begin{array}{c}\mathrm{C}_{\mathrm{U}} \\
(\mathrm{M})\end{array}$ \\
\hline ApoDREAM-C & $0.35 \pm 0.01$ & $0.30 \pm 0.01$ & 1.2 \\
\hline $\mathrm{Mg}^{2+} \mathrm{DREAM}-\mathrm{C}$ & $0.31 \pm 0.01$ & $0.37 \pm 0.01$ & 0.8 \\
\hline $\mathrm{Ca}^{2+} \mathrm{DREAM-C}$ & $3.45 \pm 0.17$ & $0.87 \pm 0.04$ & 4.0 \\
\hline $\mathrm{Ca}^{2+} \mathrm{Mg}^{2+} \mathrm{DREAM}-\mathrm{C}$ & $3.60 \pm 0.17$ & $0.94 \pm 0.04$ & 3.8 \\
\hline $\begin{array}{l}\Delta \mathrm{G}_{\mathrm{U}}, \mathrm{m}_{\mathrm{U}}, \text { and } \mathrm{C}_{\mathrm{U}} \text { represent the unfolding free energy change describes, the free } \\
\text { energy dependent on the denaturant concentration, and denaturant concentration } \\
\text { of half unfold from native to unfolding state (subscript } \mathrm{U}) .\end{array}$ \\
\hline
\end{tabular}

\section{Discussion}

On the basics of the $\mathrm{Ca}^{2+}$ and $\mathrm{Mg}^{2+}$ binding properties, the canonical EF- hand binding sites can be divided into two classes: regulatory sites and structural sites. The structural sites bind $\mathrm{Mg}^{2+}$ at the resting $\mathrm{Ca}^{2+}$ concentration and $\mathrm{Mg}^{2+}$ is replaced by $\mathrm{Ca}^{2+}$ in response to an intracellular increase in the $\mathrm{Ca}^{2+}$ concentration. On the other hand, the regulatory binding sites are $\mathrm{Ca}^{2+}$ specific with a moderate affinity for $\mathrm{Ca}^{2+}$. $\mathrm{The}^{\mathrm{Mg}^{2+}}$ association to EF-hands was shown to be physiologically important as it modulates the EFhand affinity for $\mathrm{Ca}^{2+}$, stabilizes protein structure, and in DREAM directly alters protein 
function (Yamniuk et al. 2004; Wingard et al., 2005; Peshenko and Dizhoor 2006). The metal biding specificity of individual EF-hands cannot be determined based on the protein sequence, because the side chain of the $12^{\text {th }}$ loop residue is dynamically rotated. EF-hand loop flexibility and electrostatic potential are likely to contribute to the observed specificity (Henzl et al., 1998).

Both classes of EF-hand sites have been found among members of NCS family but the role of individual EF-hands varies among the NCS subfamilies. The EF-hand 1 is not functional among all members of this family, whereas the EF-hands 2, 3, and 4 can be classified either as a structural or regulatory binding sites (Osawa et al., 2005). Visinin-like protein 1 binds functionally to $\mathrm{Mg}^{2+}$ at EF-hand 3 in the absence of $\mathrm{Ca}^{2+}$, whereas upon increase in the $\mathrm{Ca}^{2+}$ concentration, the EF-hand 2 and EF-hand 3 binds $\mathrm{Ca}^{2+}$ cooperatively while EF- hand 4 is nonfunctional. In guanylyl cyclase activating protein-1, EF-hands 2, 3, and 4 are occupied by either $\mathrm{Mg}^{2+}$ or $\mathrm{Ca}^{2+}$, with $\mathrm{Mg}^{2+}$ binding to EF-hands 2 and 3 being essential for activation of photoreceptor membrane guanylyl cyclase (Peshenko and Dizhoor 2006). On the other hand, EF-hands 2 and 3 in neuronal calcium sensor 1 function as the structural binding sites, whereas EF-hand 4 binds calcium specifically (Aravind et al., 2008).

Among the members of the KChIP subclass, KChIP2, KChIP4 and DREAM bind selectively to $\mathrm{Mg}^{2+}$ at EF-hand 2 due to the substitution of Glu by Asp in the $12^{\text {th }}$ position, whereas the EF-hand 2 is nonfunctional in KChIP1 due to the Thr substitution in the position of the $3^{\text {rd }}$ and $5^{\text {th }}$ ligand. In all KChIPs, EF-hand 3 and 4 are regulatory sites that specifically bind $\mathrm{Ca}^{2+}$. In DREAM, EF hand 3 and 4 bind $\mathrm{Ca}^{2+}$ cooperatively with a $\mathrm{K}_{\mathrm{d}}$ value of 1 - 10 M (Osawa et al., 2001; Osawa et al., 2005; Ames et al., 2006; Lusin et al., 
2008). According to the results of NMR spectroscopy, it was proposed that $\mathrm{Mg}^{2+}$ association to EF-hand 2 stabilizes DREAM conformation and promotes DREAM association to the downstream regulatory element (DRE) sequence (Osawa et al., 2005). However, no significant changes in the protein secondary or tertiary structure takes place due to the $\mathrm{Mg}^{2+}$ association to EF-hand 2, as evident from the absence of W169 emission enhancement and/or increase in the $\alpha$-helical contain upon $\mathrm{Mg}^{2+}$ association to ApoDREAM (Osawa et al., 2005; Lusin et al., 2008; Pham et al. 2015; Gonzalez et al., 2016).

The ITC data presented here clearly show that the truncated form of DREAM that lacks the EF-hand 2, binds to $\mathrm{Mg}^{2+}$ with a sub-micromolar affinity, $\mathrm{K}_{\mathrm{d} 1}=180 \pm 10 \mu \mathrm{M}$. The association of $\mathrm{Mg}^{2+}$ to ApoDREAM-C alters the affinity of C-terminal domain EFhands for $\mathrm{Ca}^{2+}$ as the overall equilibrium dissociation constant increases from $520 \mathrm{nM}$ in the absence of $\mathrm{Mg}^{2+}$ to $770 \mathrm{nM}$ in the presence of $\mathrm{Mg}^{2+}$, respectively. The modulation of the affinity of EF-hand binding sites for $\mathrm{Ca}^{2+}$ by $\mathrm{Mg}^{2+}$ was observed previously in calmodulin and troponin $\mathrm{C}$ and attributed to the reduction of the on rate for $\mathrm{Ca}^{2+}$ binding (Gifford et al., 2007).

In addition, $\mathrm{Mg}^{2+}$ association alters DREAM-C secondary and tertiary structure, as evident from the increase in the $\alpha$-helical structure, enhancement of W169 emission, and a hypsochromic shift of the emission spectra in the nile red:Mg ${ }^{2+}$ DREAM-C complex compared to nile:red:ApoDREAM-C complex. The relatively modest increase in the $\alpha$ helical structure and the small enhancement of the nile red emission in $\mathrm{Mg}^{2+}$ DREAM-C indicates that the structural changes triggered by $\mathrm{Mg}^{2+}$ association to EF-hand 3 or EFhand 4 are minor compared to the conformational changes triggered by the $\mathrm{Ca}^{2+}$ binding. 
This is commonly observed in the EF-hand proteins as the absence of the structural transition due to $\mathrm{Mg}^{2+}$ association to EF-hands is commonly attributed to the six-fold coordination of $\mathrm{Mg}^{2+}$ compared to seven-fold ligation geometry for $\mathrm{Ca}^{2+}$ ions (Cates et al., 1999). The $\mathrm{Mg}^{2+}$ association to the isolated C-terminal domain of DREAM (residues 154 - 256) was reported previously by Craig et al (Craig et al., 2002) using micro-electrospray ionization spectrometry. The authors proposed that $\mathrm{Mg}^{2+}$ binds to EF-hand 3 or EF-hand 4 in the C-terminal domain with an affinity that is comparable to the results reported in this study.

Importantly, the structural changes induced by $\mathrm{Mg}^{2+}$ association to ApoDREAM$\mathrm{C}$ are reversed by addition of $\mathrm{Ca}^{2+}$ suggesting that DREAM-C can populate three distinct structures ApoDREAM-C, $\mathrm{Mg}^{2+}$ DREAM-C and $\mathrm{Ca}^{2+}$ DREAM-C. Such reversal of $\mathrm{Mg}^{2+}$ induced structural changes have been reported for another member of NCS family, neuronal calcium sensor 1 (Jeromin et al., 2004). Indeed, $\mathrm{Mg}^{2+}$ binding to the Apo form of NCS-1 leads to a comparable increase in the tryptophan emission and the subsequent $\mathrm{Ca}^{2+}$ addition to $\mathrm{Mg}^{2+}$ bound NCS-1 resulted in the Trp emission properties that were identical to those of $\mathrm{Ca}^{2+}$ bound NCS-1 as observed here for DREAM-C (Jeromin et al., 2004). Unlike DREAM, NCS-1 binds $\mathrm{Mg}^{2+}$ ion at both EF-hands 2 and 3 and the $\mathrm{Mg}^{2+}$ ion is replaced by $\mathrm{Ca}^{2+}$ as increased intracellular $\mathrm{Ca}^{2+}$ concentration suggesting that both sites belongs to the structural sites. Considering a high sequence similarity between the Cterminal domains of members of neuronal calcium sensor family, I hypothesize that the EF-hand 3 in DREAM-C binds $\mathrm{Mg}^{2+}$ ion with a high affinity. Since the EF-hand 3 is a regulatory site that does not bind $\mathrm{Mg}^{2+}$ in the full length DREAM, the results presented here suggest that the inter-domain interactions between the $\mathrm{C}$ - and $\mathrm{N}$-terminal domains 
modulate the metal binding properties of the EF-hand 3. The NMR structure of the DREAM protein reveals numerous contacts between the exiting $\alpha$-helix of EF-hand 2 and the residues from EF-hand 3, including a hydrogen bond between N136 and a carbonyl oxygen of D176 (+Y ligand in EF-hand 3) (previously described in chapter 1). The extensive inter-domain contacts include hydrophobic interactions between residues F133, L1733, I190, and W169 contribute to the overall stability of DREAM protein but may also modulate the $\mathrm{Ca}^{2+}$ binding properties of the EF-hand 3. Indeed, I speculate that in ApoDREAM-C, without contacts between EF-hand 2 and 3, allows an increase in flexibility of the EF-hand 3, which increases the affinity of the EF-hand 2 for $\mathrm{Mg}^{2+}$ in the absence of $\mathrm{Ca}^{2+}$.

\section{Summary}

The ITC data determined for association of $\mathrm{Ca}^{2+}$ to ApoDREAM-C shows that the binding affinity for $\mathrm{Ca}^{2+}$ association to EF-hand 3 and 4 in DREAM-C is consistent with that determined previously for DREAM (residues 65 - 256) (Osawa et al., 2005). However, the association of $\mathrm{Mg}^{2+}$ to ApoDREAM-C alters the affinity of C-terminal domain EFhands for $\mathrm{Ca}^{2+}$ as the overall equilibrium dissociation constant increases from $520 \mathrm{nM}$ in the absence of $\mathrm{Mg}^{2+}$ to $770 \mathrm{nM}$ in the presence of $\mathrm{Mg}^{2+}$, respectively. I have also shown that association of $\mathrm{Mg}^{2+}$ alters the DREAM-C secondary and tertiary structure as evident from the increase in the $\alpha$-helical structure. Importantly, the structural changes induced by $\mathrm{Mg}^{2+}$ association to ApoDREAM-C are reversed by addition of $\mathrm{Ca}^{2+}$ suggesting that

DREAM-C can populate three distinct structures ApoDREAM-C, $\mathrm{Mg}^{2+} \mathrm{DREAM}-\mathrm{C}$ and $\mathrm{Ca}^{2+}$ DREAM-C. These results also suggest that the inter-domain interactions between the 
C-terminal domain and $\mathrm{N}$-terminal domain is modulated by the metal binding properties of the EF-hand 3 in DREAM. 


\section{MOLECULAR INSIGHTS INTO DREAM AND PRESENILIN 1 C-TERMINAL FRAGMENT INTERACTIONS}

(The work described in this chapter was published in K., Pham and J., Miksovska (2016), FEBS letters, 590(8):1114-1122)

\subsection{Background and significance}

Downstream regulatory element antagonist modulator (DREAM), also known as calsenilin or KChIP3, belongs to the neuronal calcium sensor (NCS) family (Burgoyne et al., 2004). DREAM carries four EF-hand $\mathrm{Ca}^{2+}$ binding motifs (EF-hand) of which EFhands 3 and 4 functionally bind to $\mathrm{Ca}^{2+}$ with high affinity $\left(\mathrm{K}_{\mathrm{d}} \sim 1-10 \mu \mathrm{M}\right)$, whereas EFhand 2 preferentially interacts with $\mathrm{Mg}^{2+}\left(\mathrm{K}_{\mathrm{d}} \sim 13 \mu \mathrm{M}\right)$ and EF-hand 1 is inactive (Osawa et al., 2005). The NMR structure of $\mathrm{Ca}^{2+}$ bound mouse DREAM (PDB code: 2JUL, Figure 6.1) reveals that its amino- and carboxy-terminal domains are arranged in tandem and stabilized by a cluster of hydrophobic residues F114, F135, W169, F171, F218, and F235 (Lusin et al., 2008). A dynamic loop connecting EF-hands 3 and 4 in DREAM was suggested to be involved in the binding and recognition of target proteins (Yu et al., 2007). Several physiological functions of DREAM have been reported including prodynorphin gene transcriptional repression (Carrion et al., 1999; Osawa et al., 2001), potassium channel activity modulation (An et al., 2000), and enhancement of calcineurin activation by binding to calmodulin (Ramachandran et al., 2012). Interactions between DREAM and the carboxy-terminal fragment (CTF) of presenilin (PS) 1 and PS2 were proposed to be involved in many biological functions including amyloid precursor protein processing, beta amyloid peptide generation, neuronal apoptosis, calcium signaling in the endoplasmic recticulum, and regulation of N-cadherin processing (Buxbaum et al., 1998; Leissring et 
al., 2000; Jo et al., 2001; Lilliehook et al., 2002; Jo et al., 2004; Jo et al., 2005; Fedrizzi et al., 2008; Jang et al., 2011).

PS1 is a catalyst component of the $\gamma$-secretase complex which mediates cleavage of amyloid precursor protein to produce $\mathrm{A} \beta$ peptides. In order to activate $\gamma$-secretase complex, PS1 undergoes endoproteolysis within the hydrophobic sequence T291-A299 forming the amino-terminal fragment (NTF) and CTF (Podlisny et al., 1997; Li et al., 2014). Initially, it was suggested that PS1 consists of 6 transmembrane helices at NTF and one to three transmembrane helices at CTF (Lehmann et al., 1997; Dewji and Singer, 1997; Nakai et al., 1999; Li and Greenwald, 1998; Spasic et al., 2006). The NMR structure of human PS1-CTF (PDB entry: 2KR6, Figure 6.1) reveals that PS1-CTF consists of a long unstructured loop (residues 300 - 354, not shown in Figure 6.1) and a soluble helix $\beta$ (HL $\beta$, residues 355 - 369) at the amino-terminus. The core of PS1-CTF contains two transmembrane helices HL7 (residues 384 - 398) and HL8 (residues 407 - 428). The carboxyl HL9 (residues 441- 462) is an intramembranous helix, which is divided into two perpendicular helices due to the presence of proline 455 . The last four residues $(463-467)$ in PS1 are unstructured (Sobhanifar et al., 2010). NMR studies and molecular dynamic simulations of PS1-CTF incorporated in lipid environments demonstrated that residues in HL9 are exposed to the surrounding aqueous environment and experience greater dynamics (Sobhanifar et al., 2010). Recently, the cryo-electron microscopy structure of PS1 in human $\gamma$-secretase complex revealed that PS1 is comprised of nine helices, which are loosely organized and exhibit considerable flexibility (Bai et al., 2015).

A thorough understanding of the molecular mechanism of DREAM and PS1 interactions requires the identification of binding sites between DREAM and PS1-CTF as 
well as characterization of the role of $\mathrm{Ca}^{2+}$ and/or $\mathrm{Mg}^{2+}$ on DREAM and PS1-CTF complex formation. In this report, binding interfaces between DREAM and PS1-CTF were identified by determining the binding affinity for DREAM, DREAM with non-functional EF hand 3 or 4, and chimeric forms of DREAM association to synthetic peptides derived from residues 445 - 467 (HL9) and residues 355 - 369 (HL $\beta$ ) of PS1. The residues comprising HL9 were chosen because previous studies demonstrated that they interact with DREAM and a homologous protein KChIP4 in a yeast two-hybrid system (Buxbaum et al., 1998; Morohashi et al., 2002). In addition, the residues composing HL $\beta$ are in the region (residues 263 - 407) previously shown to interact with catenin, but have not been tested with DREAM (Zhou et al., 1997; Buxbaum et al., 1998). Since transmembrane helices 7 and 8 are composed of highly hydrophobic natural amino acid residues and were shown to be stably integrated in a lipid bilayer (Sobhanifar et al., 2010; Bai et al., 2015), these structural elements were not included in this study.

\subsection{Results}

\subsubsection{Computational protein-protein docking of DREAM and PS1-CTF}

Docking complex of $\mathrm{Ca}^{2+}$ DREAM:PS1-CTF, as shown in Figure 6.1, reveals that

the assembly of $\mathrm{Ca}^{2+}$ DREAM and PS1-CTF involves $\pi-\pi$ interactions in which the aromatic ring of F252 in DREAM stacks against the aromatic rings of F462 and F465 from HL9. The complex is further stabilized by a cation- $\pi$ interaction in which the planar guanidinium group of R200 interacts with the aromatic ring of F465 from HL9. Moreover, F462 and F465 from HL9 insert into a cavity formed by hydrophobic residues M191, I194, 

surface in Fig 6.1).

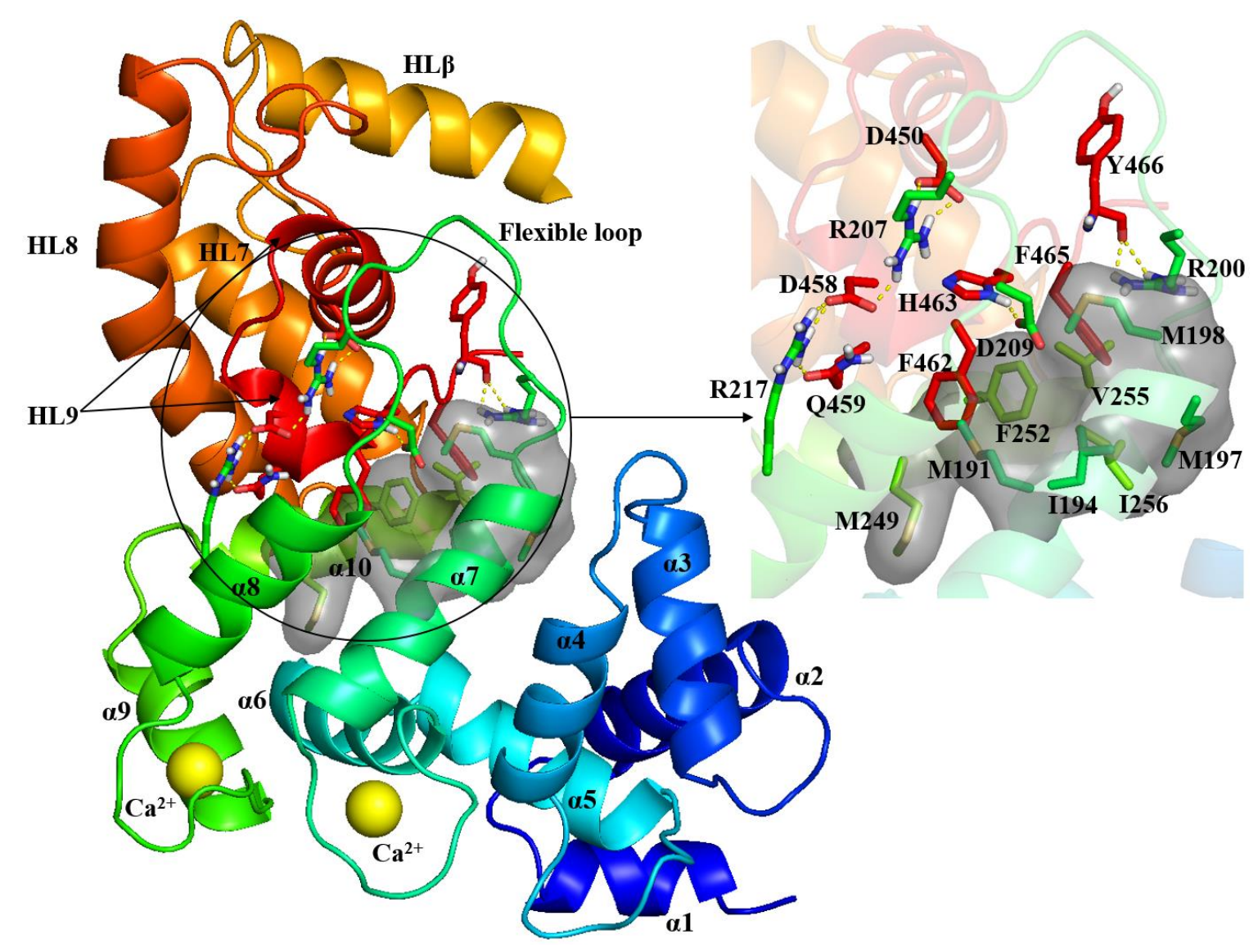

Figure 6.1: Left: Protein-protein docking complex of $\mathrm{Ca}^{2+}$ DREAM (PDB: 2JUL, molecule 1) and PS1-CTF (PDB: 2KR6, molecule 1). DREAM (residues 76 - 256) contains $10 \alpha$ helices respectively labeled $\alpha 1-\alpha 10$, and four EF-hands: EF-1, EF-2, EF-3, and EF-4. Two $\mathrm{Ca}^{2+}$ ions (yellow sphere) are bound at EF-3 and EF-4 loops in DREAM. PS1-CTF comprises two trans-membrane helices: HL7 and HL8, a solvent exposed helix HL $\beta$, and an intramembranous HL9 which is divided into two perpendicular helices. Right: Detailed presentation of the binding interfaces between $\mathrm{Ca}^{2+}$ DREAM and PS1-CTF. Amino acid residues from DREAM and PS1 are colored in green and red, respectively. Yellow dot lines represent for hydrogen bond interactions.

The residue F465 in PS1 plays an important role not only for interaction with DREAM, but also for interaction with anterior pharynx defective-1 (APH1) in the $\gamma$ secretase complex (Bai et al., 2015). Furthermore, assembly of DREAM and PS-CTF 
involves formation of salt bridges between R207 in DREAM and D450 and D458 from HL9. An additional salt bridge is found between R217 in DREAM and residue D458 from HL9. In addition, hydrogen bond interactions are found between the side chains of four pairs of residues: D450-R207, D458-R217, Q459-R217, and H463-D209, where D450, D458, Q459, and H463 are residues from PS1 and R207, R209, and R217 are residues from DREAM (Figure 6.1). Additional hydrogen bond is found between the carbonyl oxygen of Y466 from PS1 and side chain of R200 from DREAM. This hydrogen bond likely stabilizes side chain of R200 in order to direct its guanidinium group to the aromatic ring of F465 from PS1.

\subsubsection{Region of PS1-CTF in contact with DREAM}

In order to determine which regions of PS1-CTF are in contact with DREAM and investigate the impact of $\mathrm{Mg}^{2+} / \mathrm{Ca}^{2+}$ on DREAM and PS1-CTF complex formation, titrations of DREAM, DREAM with inactivated EF-hand 3 or 4, DREAM-C, and DREAM chimeric constructs into PS1 derived peptides were conducted in the presence of $1 \mathrm{mM}$ $\mathrm{Ca}^{2+}$ and/or $5 \mathrm{mM} \mathrm{Mg}^{2+}$. The titration curve for DREAM binding to HL9 is shown in Figure 6.2 and the dissociation constants are summarized in Table 6.1.

Table 6.1: Dissociation constant $\left(\mathrm{K}_{\mathrm{d}}\right)$ of DREAM binding to HL $\beta$ and HL9 in PS1-CTF

\begin{tabular}{|l|c|c|}
\hline \multirow{2}{*}{\multicolumn{1}{|c|}{ DREAM }} & \multicolumn{2}{c|}{$\mathrm{K}_{\mathrm{d}}(\mu \mathrm{M})$} \\
\cline { 2 - 2 } & HL9 & HL $\beta$ \\
\hline $\mathrm{Ca}^{2+}$ DREAM & $0.6 \pm 0.1$ & \\
\hline $\mathrm{Ca}^{2+} / \mathrm{Mg}^{2+}$ DREAM & $0.7 \pm 0.1$ & \multirow{2}{*}{ No binding } \\
\hline $\mathrm{Mg}^{2+}$ DREAM & $29 \pm 2$ & \\
\hline ApoDREAM & $183 \pm 12$ & \\
\hline
\end{tabular}



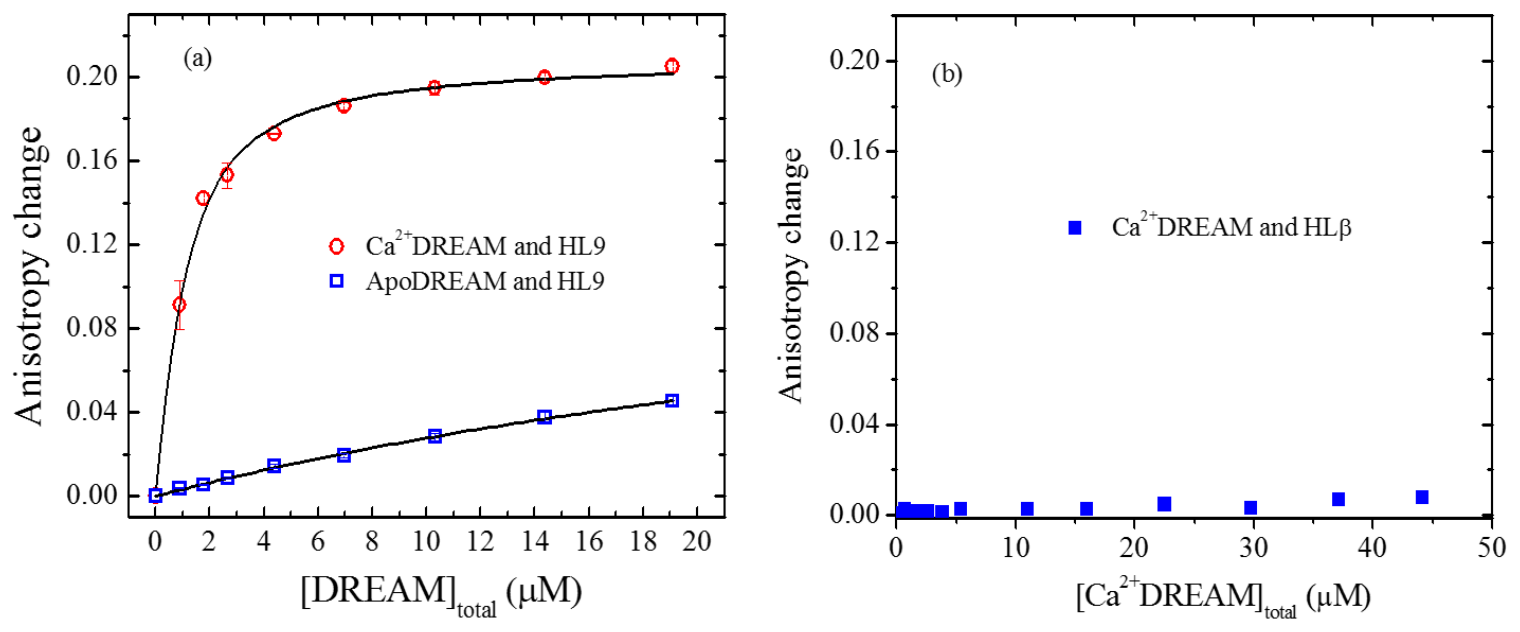

Figure 6.2: Titration curves for DREAM binding to HL9 (a) and HL $\beta$ (b) of PS1-CTF. Solid lines correspond to the best fit using equation 3.63 in section 3.5.2.

HL9 binds to $\mathrm{Ca}^{2+}$ DREAM and $\mathrm{Mg}^{2+} \mathrm{Ca}^{2+} \mathrm{DREAM}$ with a similar $\mathrm{K}_{\mathrm{d}}$ of $0.6 \mu \mathrm{M}$. In the absence of $\mathrm{Ca}^{2+}$, a weak interaction was determined for binding of HL9 to ApoDREAM $\left(\mathrm{K}_{\mathrm{d}}=183 \pm 12 \mu \mathrm{M}\right)$, whereas the $\mathrm{K}_{\mathrm{d}}$ value for HL9 association with $\mathrm{Mg}^{2+}$ DREAM was found to be $29 \pm 2 \mu \mathrm{M}$. Unlike HL9, the HL $\beta$ does not form a complex with DREAM.

\subsubsection{HL9 associates with DREAM at the carboxy-terminal domain}

Since our titration data indicate that HL9 associates with DREAM in the presence of $\mathrm{Ca}^{2+}$ and the $\mathrm{Ca}^{2+}$ binding sites are located at DREAM carboxy-terminal domain, I tested whether DREAM-C provides a binding site for HL9. Titration data of HL9 binding to DREAM-C (Figure 6.3) demonstrate that HL9 binds to $\mathrm{Ca}^{2+}$ DREAM-C with a $\mathrm{K}_{\mathrm{d}}$ of $11 \pm$ $1 \mu \mathrm{M}$ and a similar $\mathrm{K}_{\mathrm{d}}$ was determined for association of HL9 with $\mathrm{Ca}^{2+} \mathrm{Mg}^{2+}$ DREAM-C. On the other hand, a weaker affinity was obtained for HL9 binding to $\mathrm{Mg}^{2+}$ DREAM-C $\left(\mathrm{K}_{\mathrm{d}}\right.$ $\sim 146 \pm 12 \mu \mathrm{M}$ ) and no interaction was observed in the Apo form (Table 6.2). 


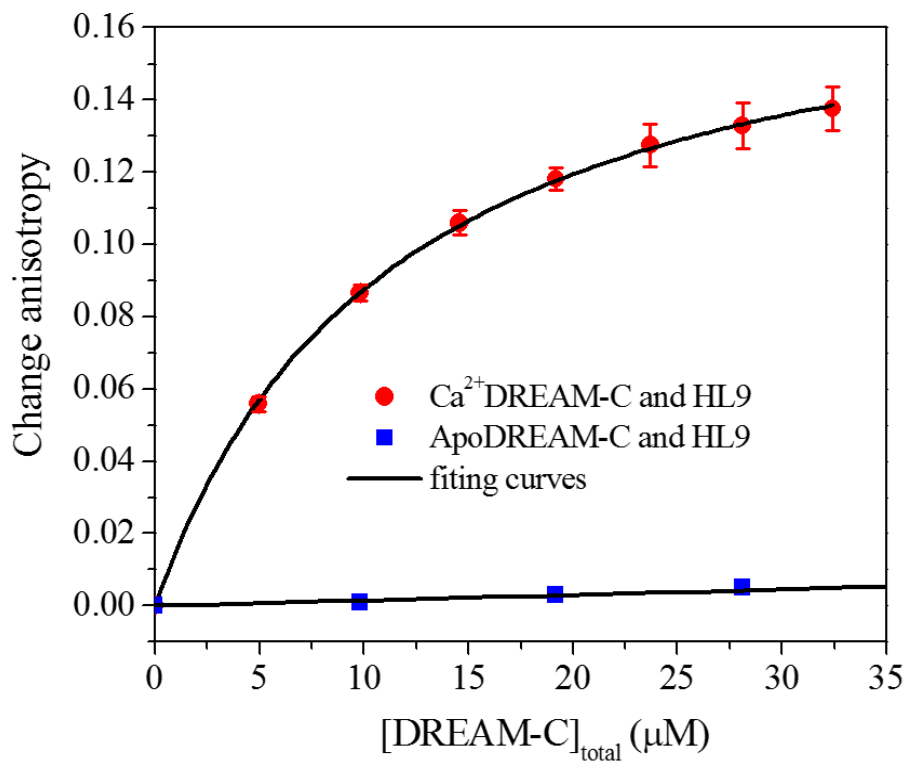

Figure 6.3: Titration curves for DREAM-C binding to HL9 of PS1-CTF in the presence and absence of $\mathrm{Ca}^{2+}$. Solid lines correspond to the best fit using equation 3.63 in section 3.5.2.

Table 6.2: Dissociation constant $\left(\mathrm{K}_{\mathrm{d}}\right)$ of DREAM-C binding to HL9 in PS1-CTF

\begin{tabular}{|l|c|}
\hline \multicolumn{1}{|c|}{ Protein } & $\mathrm{K}_{\mathrm{d}}(\mu \mathrm{M})$ \\
\hline $\mathrm{Ca}^{2+}$ DREAM-C & $11 \pm 1$ \\
\hline $\mathrm{Ca}^{2+} / \mathrm{Mg}^{2+}$ DREAM-C & $9 \pm 1$ \\
\hline $\mathrm{Mg}^{2+}$ DREAM-C & $146 \pm 12$ \\
\hline ApoDREAM-C & No binding \\
\hline
\end{tabular}

\subsubsection{Residues 200-207 in the DREAM flexible loop mediate HL9 association}

Bioinformatics analysis of the carboxy-terminal domain of presenilin-interacting proteins (DREAM and KChIP4) and a non-presenilin-interacting protein, such as NCS1 (Braunewell, 2005) suggests that the basic amino acid residues 200RHTYPILR207 in the flexible loop connecting $\alpha 7$ and $\alpha 8$ ( $\alpha 7 / \alpha 8$ loop) of DREAM may play a role in regulating the association of HL9 (Figure 6.4). 


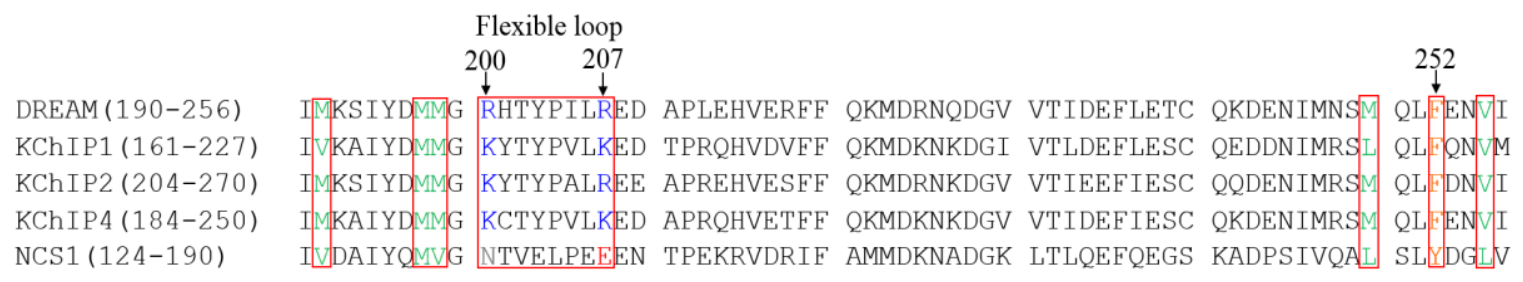

Figure 6.4: Sequence alignment of 66 amino acid residues in the carboxyl-terminus of mouse DREAM with various members of KChIPs subfamily and NCS1 protein using Clustal Omega program. The conserved hydrophobic residues at the binding interface with HL9 are highlighted in green. The conserved basic residues at positions 200 and 207 in the flexible loop of various members in KChIP subfamily are highlighted in blue. The neutral residue and the acidic residue of NCS1 corresponding to positions 200 and 207 in flexible loop are highlighted in grey and red, respectively. Aromatic residues corresponding to $\pi$ $\pi$ stacking interaction with PS1 are in orange.

To test whether these residues functionally mediate interaction with HL9, titrations of DREAM fused with NCS1 flexible loop (residues 200NTVELPEE207) were conducted. The titration curve for HL9 binding to $\mathrm{Ca}^{2+}$ DREAM-NCS1 (Figure 6.5a) demonstrates a lower affinity $\left(\mathrm{K}_{\mathrm{d}}=38 \pm 4 \mu \mathrm{M}\right)$ and a negligible interaction was, observed for binding of HL9 to ApoDREAM-NCS1.
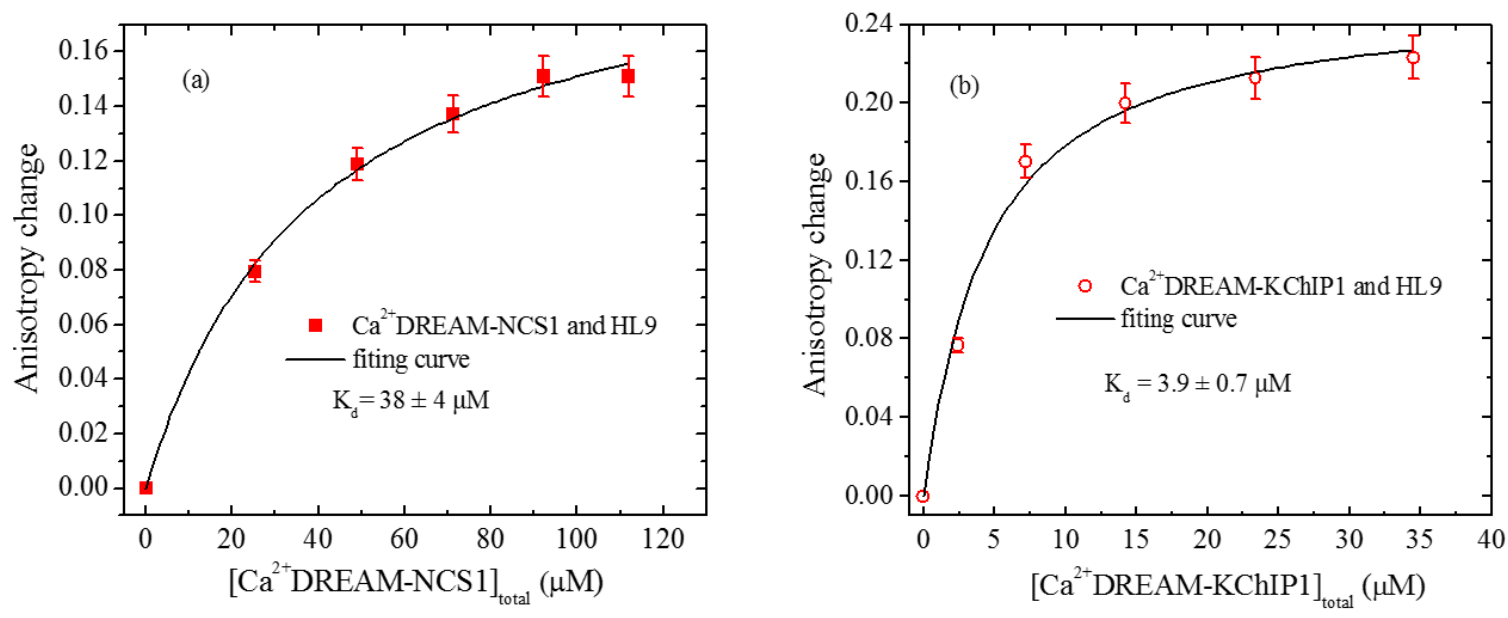

Figure 6.5: Titration curves for HL9 binding to $\mathrm{Ca}^{2+}$ DREAM-NCS1 (a) and $\mathrm{Ca}^{2+}$ DREAMKChIP1 (b). Solid lines correspond to the best fit using equation 3.63 in section 3.5.2.

Since residues in the $\alpha 7 / \alpha 8$ loop are conserved among various members of KChIP subfamily, I have also fused KChIP1 loop (200్TYPVLㅌ207) into $\alpha 7 / \alpha 8$ loop of 
DREAM and determined dissociation constant for binding to HL9 (Figure 6.5b). Interestingly, the HL9 binds to DREAM-KChIP1 with a lower affinity compared to that of $\operatorname{DREAM}\left(\mathrm{K}_{\mathrm{d}}=3.9 \pm 0.7 \mu \mathrm{M}\right)$.

\subsubsection{Binding of $\mathrm{Ca}^{2+}$ at EF-hand 3 functionally modulates the interaction of DREAM with HL9}

To investigate the mechanism by which $\mathrm{Ca}^{2+}$ binding to EF-hands 3 and 4 in DREAM mediates DREAM and HL9 interactions, binding of HL9 to DREAM-E234Q and DREAM-E186Q were studied. Titration data demonstrate that HL9 binds to DREAME234Q in the presence of $\mathrm{Ca}^{2+}$ and $\mathrm{Ca}^{2+} / \mathrm{Mg}^{2+}$ with a $\mathrm{K}_{\mathrm{d}}$ of $8 \mu \mathrm{M}$ (Figure 6.6a), whereas a weaker binding $\left(\mathrm{K}_{\mathrm{d}}=32 \mu \mathrm{M}\right)$ was observed for DREAM-E186Q mutant (Figure 6.6b).
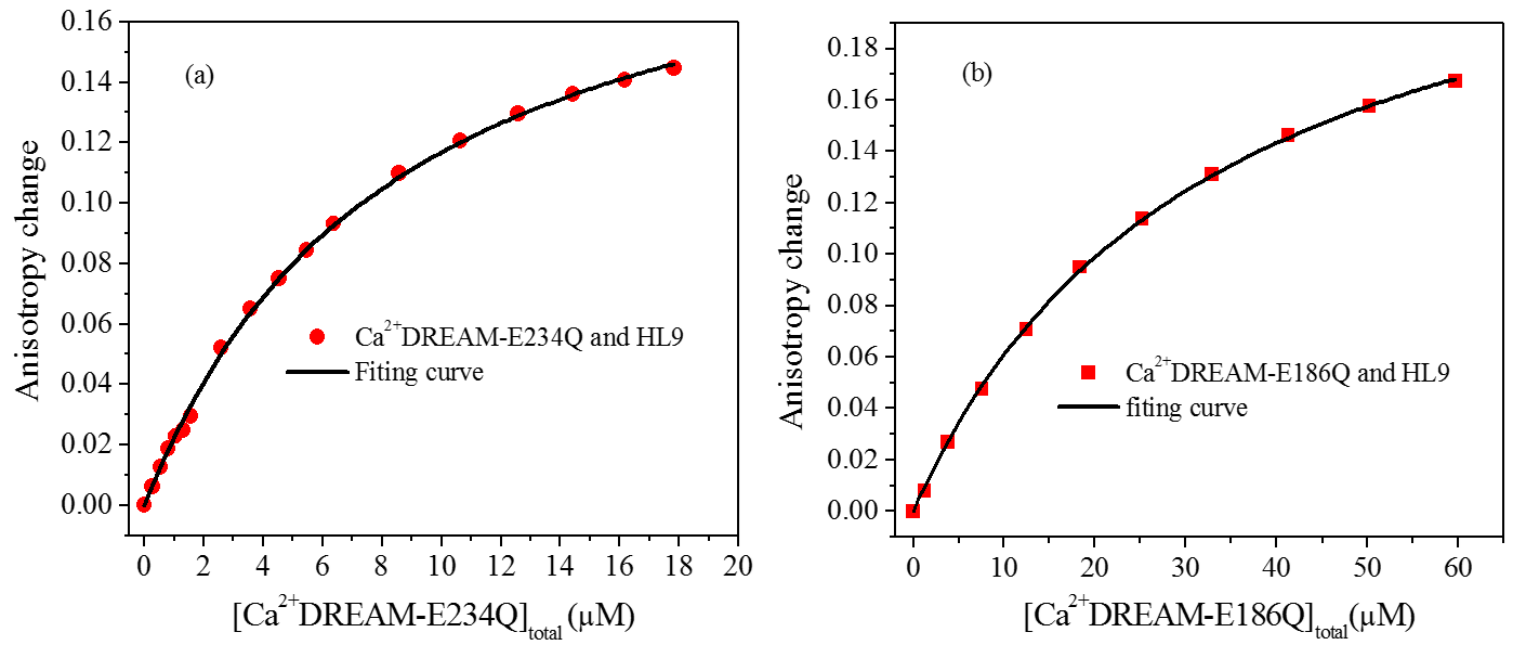

Figure 6.6: Titration curves for HL9 binding to $\mathrm{Ca}^{2+}$ DREAM-E234Q (a) and $\mathrm{Ca}^{2+}$ DREAM-E186Q (b). Solid lines correspond to the best fit using 3.63 in section 3.5.2.

These results also show that weak interaction was determined for HL9 binding to $\mathrm{Mg}^{2+}$ DREAM-E234Q and $\mathrm{Mg}^{2+}$ DREAM-E186Q. In addition, the HL9 exhibits very weak interaction with the Apo form of these DREAM mutants (Table 6.3). 
Table 6.3: Dissociation constant $\left(\mathrm{K}_{\mathrm{d}}\right)$ for HL9 binding to DREAM EF-hand mutants

\begin{tabular}{|l|c|c|}
\hline \multirow{2}{*}{} & \multicolumn{2}{|c|}{$\mathrm{K}_{\mathrm{d}}(\mu \mathrm{M})$} \\
\cline { 2 - 3 } & $\begin{array}{c}\text { DREAM-E234Q } \\
\text { (active EF-hand 3 })\end{array}$ & $\begin{array}{c}\text { DREAM-E186Q } \\
\text { (active EF-hand 4) }\end{array}$ \\
\hline $\mathrm{Ca}^{2+}$ & $8 \pm 1$ & $32 \pm 1$ \\
\hline $\mathrm{Ca}^{2+} \mathrm{Mg}^{2+}$ & $7 \pm 1$ & $30 \pm 2$ \\
\hline $\mathrm{Mg}^{2+}$ & $68 \pm 5$ & $55 \pm 2$ \\
\hline Apo & $146 \pm 14$ & $149 \pm 22$ \\
\hline
\end{tabular}

\subsubsection{Oligomerization states of DREAM in complex with HL9}

Since alternative oligomerization states of DREAM directly mediate the interactions with intracellular proteins and DNA and this process depends on $\mathrm{Ca}^{2+} / \mathrm{Mg}^{2+}$ occupancy at EF-hands (Osawa et al., 2001; Osawa et al., 2005; Lusin et al. 2008; Gonzalez and Miksovska, 2014), the oligomeric states of DREAM in complex with HL9 were characterized. The anisotropy decay traces for HL9 (circles) and $\mathrm{Ca}^{2+}$ DREAM:HL9 complex (squares) are shown in Figure 6.7a.

The data were fit into a two rotational correlation-time model where the fast rotational correlation time $\left(\theta_{2}\right)$ corresponds to the local rotation of FITC and the slow rotational correlation time $\left(\theta_{1}\right)$ reflects global rotation of HL9 or DREAM bound HL9 (Table 6.4). The values of local rotation of FITC probe in HL9 and in the complex of DREAM and HL9 are almost identical $\left(\theta_{2} \sim 1 \mathrm{~ns}\right)$, whereas the global rotation values increase from $2 \mathrm{~ns}$ in the case of HL9 in buffer solution to $12 \mathrm{~ns}$ and $20 \mathrm{~ns}$ when HL9 is bound to $\mathrm{Mg}^{2+}$ DREAM and $\mathrm{Ca}^{2+} \mathrm{DREAM} / \mathrm{Ca}^{2+} \mathrm{Mg}^{2+}$ DREAM, respectively. With the known molecular weight of HL9 and DREAM, the values of $\theta_{1}$ for DREAM monomer binding to one HL9 and DREAM dimer binding to two HL9 are estimated to be $11.4 \mathrm{~ns}$ 
and $22.7 \mathrm{~ns}$, respectively, using a degree of hydration of $0.43 \mathrm{ml} \mathrm{g}^{-1}$ for a spherical protein at $20{ }^{\circ} \mathrm{C}$ (Weber, 1953; Fischer et al., 2004). The experimentally determined $\theta_{1}$ value for $\mathrm{Mg}^{2+}$ DREAM and HL9 complex is $11.8 \mathrm{~ns}$ which matches well with the expected value of DREAM monomer bound to one HL9. However, the experimental $\theta_{1}$ value for HL9 in complex with $\mathrm{Ca}^{2+}$ DREAM/ $\mathrm{Ca}^{2+} \mathrm{Mg}^{2+}$ DREAM was determined to be $20 \mathrm{~ns}$, which is about $2 \mathrm{~ns}$ faster than the expected correlation time of a dimeric DREAM bound to two HL9. These results suggest that the structure of DREAM is more rigid when $\mathrm{Ca}^{2+}$ is bound to EF-hands 3 and 4.
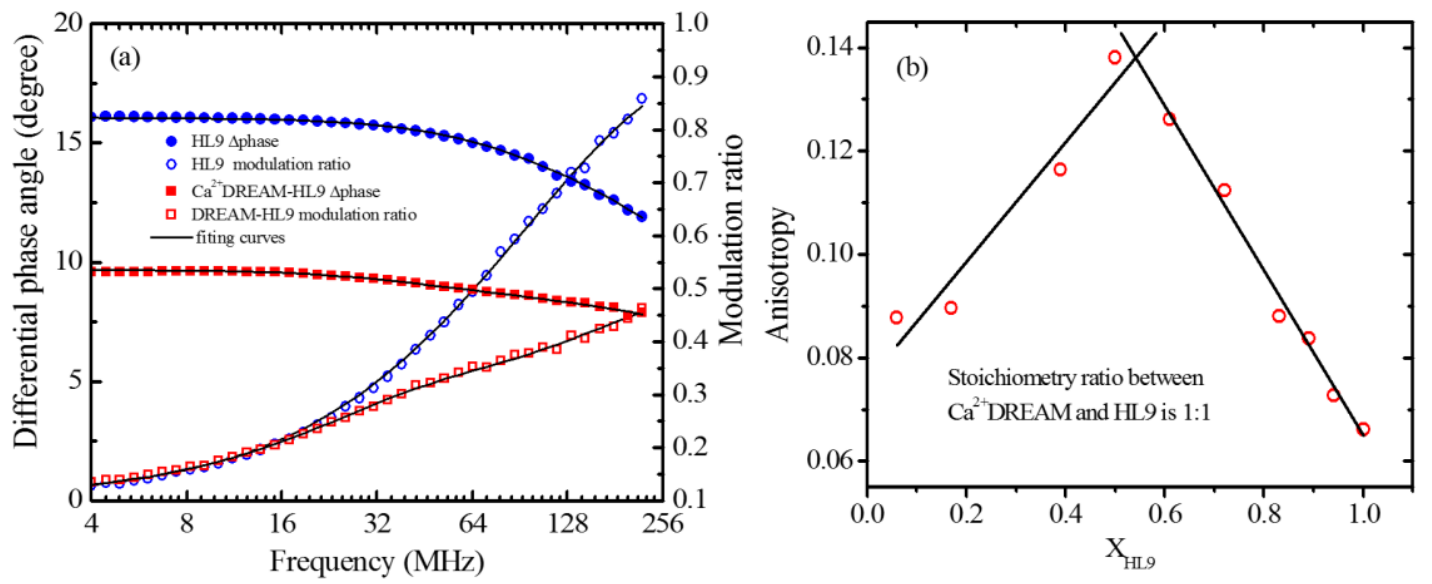

Figure 6.7: Panel (a) is time-resolved anisotropy of HL9 in buffer solution (circles) and HL9 bound $\mathrm{Ca}^{2+}$ DREAM (square). Differential phase angle and modulation ratio are shown as solid and empty symbols, respectively. Panel (b) is a Job plot curve represents a 1:1 stoichiometry for molecular association of HL9 with $\mathrm{Ca}^{2+}$ DREAM.

Furthermore, the Job plot (Figure 6.7b) shows a maximum at HL9 mole fraction ( $\mathrm{X}_{\mathrm{HL} 9}$ ) of 0.5 , what is consistent with a 1:1 stoichiometry for molecular association of HL9 with $\mathrm{Ca}^{2+}$ DREAM (Olson and Bühlmann, 2011; Furlong et al., 2013). These results further suggest that a dimeric DREAM binds to two HL9 in the presence of $\mathrm{Ca}^{2+}$ or $\mathrm{Ca}^{2+} \mathrm{Mg}^{2+}$. 
Table 6.4: Time-resolved anisotropy parameters for DREAM and HL9 complex

\begin{tabular}{|c|c|c|c|c|c|}
\hline & $\begin{array}{c}\theta_{1} \\
\text { (ns) }\end{array}$ & $r_{1}{ }^{a}$ & $\begin{array}{c}\theta_{2}{ }^{\mathrm{a}} \\
(\mathrm{ns})\end{array}$ & $\mathrm{r}_{2}^{\mathrm{a}}$ & $\chi^{2}$ \\
\hline HL9 in buffer & $1.7 \pm 0.2$ & 0.12 & 0.4 & 0.24 & 0.6 \\
\hline $\mathrm{Mg}^{2+}$ DREAM:HL9 & $11.8 \pm 2.0$ & 0.05 & 0.8 & 0.26 & 0.7 \\
\hline $\mathrm{Ca}^{2+}$ DREAM:HL9 & $20.4 \pm 0.8$ & 0.25 & 0.8 & 0.14 & 0.3 \\
\hline $\mathrm{Ca}^{2+} \mathrm{Mg}^{2+}$ DREAM:HL9 & $19.3 \pm 0.7$ & 0.26 & 0.7 & 0.13 & 0.3 \\
\hline
\end{tabular}

\subsubsection{Kinetics of HL9 association with DREAM}

Binding of HL9 to DREAM leads to an increase in emission intensity at $520 \mathrm{~nm}$ due to the fluorescence resonance energy transfer (FRET) between W169 in DREAM and FITC label on HL9 (inset in Figure 6.8).

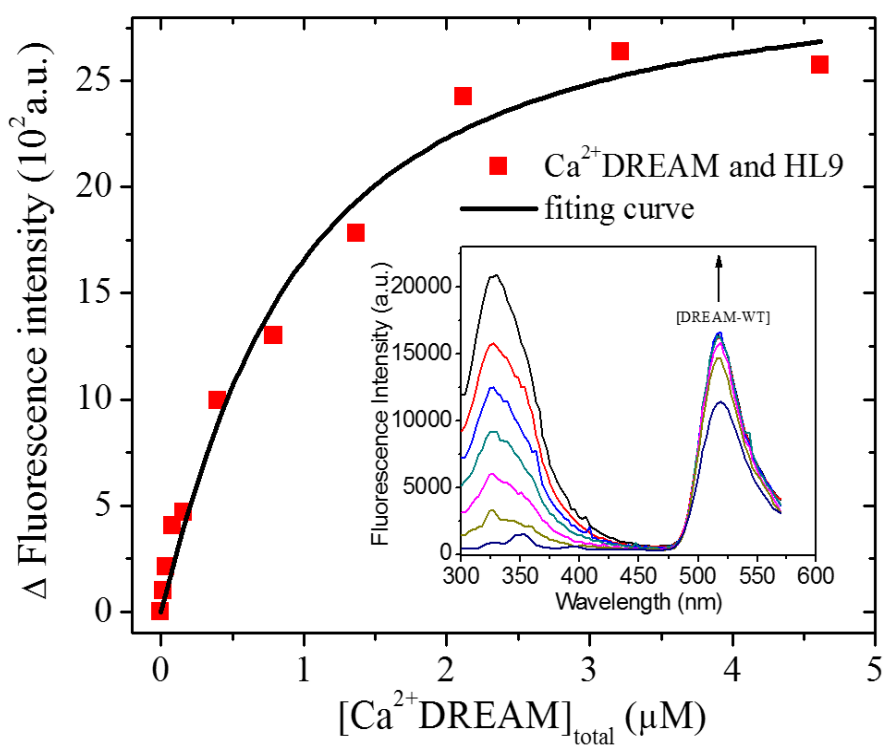

Figure 6.8: Titration curves for HL9 binding to $\mathrm{Ca}^{2+}$ DREAM by monitoring FITC fluorescence emission change at $520 \mathrm{~nm}$ (inset figure). Solid lines correspond to the best fit using equation 3.63 in section 3.5.2. The dissociation constant was determined to be 0.6 $\mu \mathrm{M}$. 
By monitoring emission intensity change at $520 \mathrm{~nm}$ as a function of DREAM concentration, dissociation constant of HL9 association with $\mathrm{Ca}^{2+}$ DREAM was determined to be $0.6 \pm 0.1 \mu \mathrm{M}$ (Figure 6.8). The determined $\mathrm{K}_{\mathrm{d}}$ in this titration is comparable with the $\mathrm{K}_{\mathrm{d}}$ previously obtained in steady-state anisotropy measurements. The results suggest an increase in the emission intensity of fluorescein probe can be used to probe kinetics of HL9 interactions with DREAM.

A representative kinetics trace for HL9 binding to $\mathrm{Ca}^{2+}$ DREAM is shown in Figure 6.9a. The dissociation rate constant ( $\mathrm{k}_{\text {off }}$ ) of HL9 from ApoDREAM was determined by addition of EDTA to $\mathrm{Ca}^{2+}$ DREAM:HL9 solution and a decrease of emission intensity at $520 \mathrm{~nm}$ was monitored (Figure 6.9b). The equilibrium dissociation constant calculated from the ratio of the dissociation rate constant $\left(\mathrm{k}_{\mathrm{off}}=1.0 \mathrm{~s}^{-1}\right)$ to association rate constant $\left(k_{\text {on }}=0.6 \times 10^{6} \mathrm{M}^{-1} \mathrm{~s}^{-1}\right)$ is in excellent agreement with the $\mathrm{K}_{\mathrm{d}}$ determined from titration experiments (Table 6.5).
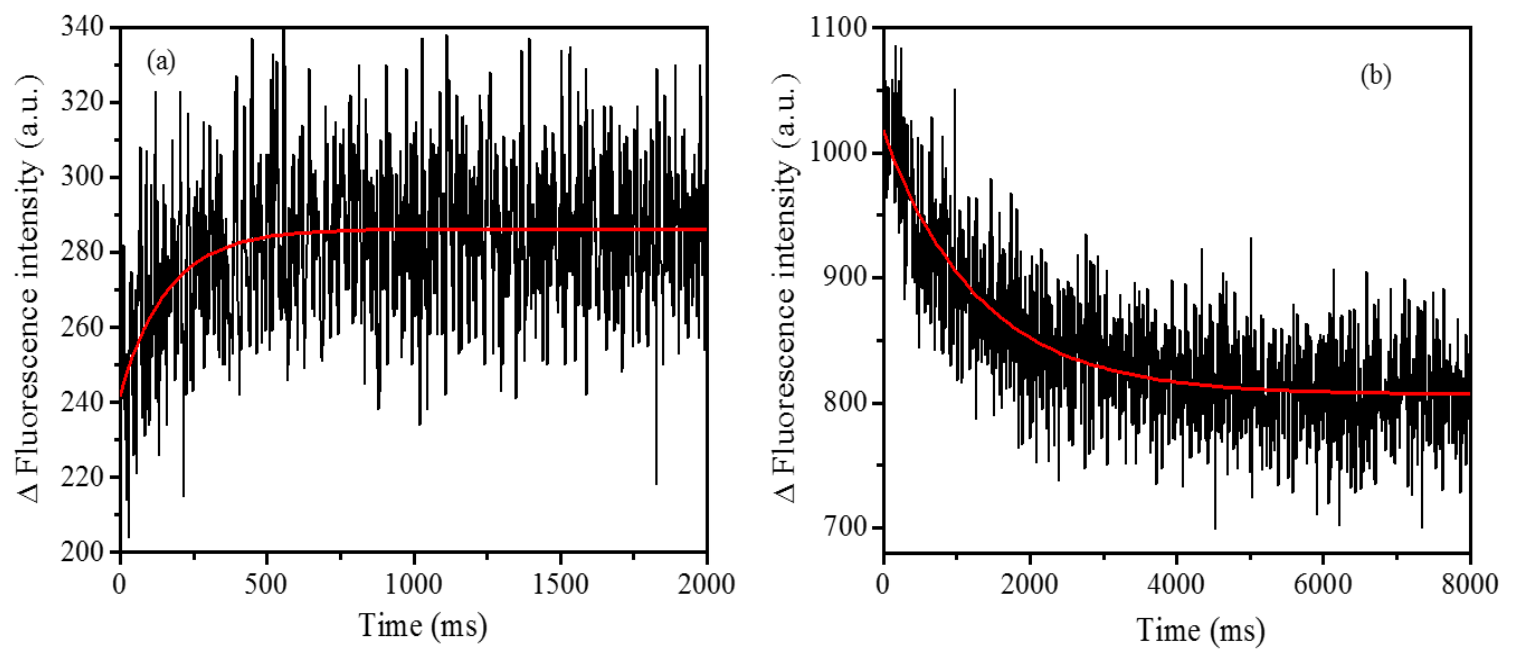

Figure 6.9: Kinetics trace for association of HL9 to $\mathrm{Ca}^{2+}$ DREAM (a) and dissociation of HL9 from ApoDREAM (b) monitored by change in the fluorescence intensity at $520 \mathrm{~nm}$. The solid line represents single exponential fitting curve. 
Table 6.5: Kinetic parameters for HL9 association with $\mathrm{Ca}^{2+}$ DREAM

\begin{tabular}{|l|l|}
\hline Activation energy $\left(\mathrm{E}_{\mathrm{a}}\right)^{(\mathrm{a})}$ & $15.9 \pm 3.1 \mathrm{kcal} \mathrm{mol}^{-1}$ \\
\hline Association rate constant $\left(\mathrm{k}_{\text {on }}\right)$ & $0.6 \times 10^{6} \mathrm{M}^{-1} \mathrm{~s}^{-1}$ \\
\hline Dissociation rate constant $\left(\mathrm{k}_{\text {off }}\right)$ & $1.0 \mathrm{~s}^{-1}$ \\
\hline $\begin{array}{l}\text { (a) } \mathrm{E}_{\mathrm{a}} \text { and error were determined from the mean and standard } \\
\text { deviations of three independent measurements, respectively. }\end{array}$ \\
\hline
\end{tabular}

Our kinetics data showed that the rate constant for the formation of $\mathrm{Ca}^{2+} \mathrm{DREAM}-$ HL9 complex is temperature and concentration dependent. The kinetics experiment was carried out at different temperatures, from 281 to $297^{\circ} \mathrm{K}$, and at various $\mathrm{Ca}^{2+}$ DREAM concentrations, from 1 to $7.5 \mu \mathrm{M}$. The apparent first order rate constants ( $\mathrm{k}_{\mathrm{obs}}$ ) for association of HL9 to each $\mathrm{Ca}^{2+}$ DREAM concentration and at each temperature was recovered and analyzed by using the first order exponential decay model. The activation energy $\left(E_{a}\right)$ was calculated from the slope $\left(-E_{a} / R\right)$ of the Arrhenius plot (Figure 6.10).

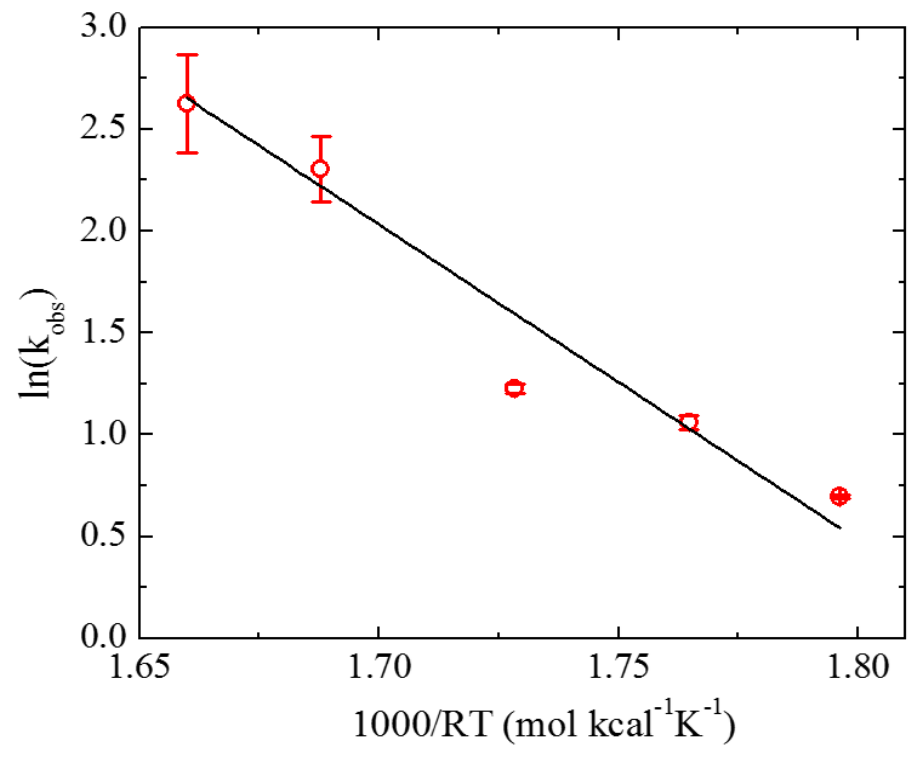

Figure 6.10: The Arrhenius plot of HL9 association with $\mathrm{Ca}^{2+}$ DREAM. $\mathrm{E}_{\mathrm{a}}$ and experimental error were determined from the mean and standard deviations of three independent measurements, respectively. 
The activation barrier for HL9 association to $\mathrm{Ca}^{2+}$ DREAM was found to be $15.9 \pm$ $3.1 \mathrm{kcal} / \mathrm{mol}$ (Table 6.5) suggesting for a large conformational change on the HL9 docking site on $\mathrm{Ca}^{2+}$ DREAM protein upon the complex formation. The high activation energy can be attributed to the structural changes, which results in a decrease of flexibility of long loop connecting $\alpha 7$ in EF-hand 3 and $\alpha 8$ in EF-hand 4 of DREAM protein (Figure 6.1).

To examine if the kinetics curves of HL9 association to $\mathrm{Ca}^{2+}$ DREAM include any diffusional components, I performed kinetics experiment with increasing in $\mathrm{Ca}^{2+} \mathrm{DREAM}$ concentration (1 to $7.5 \mu \mathrm{M})$. Apparent association rate constants $\left(\mathrm{k}_{\mathrm{obs}}\right)$ were plotted against varied $\mathrm{Ca}^{2+} \mathrm{DREAM}$ concentrations (Figure 6.11) showed that the association rate constant $\left(\mathrm{k}_{\mathrm{on}}\right)$ is $0.6 \times 10^{6} \mathrm{M}^{-1} \mathrm{~s}^{-1}$ and the dissociation rate constant $\left(\mathrm{k}_{\text {off }}\right)$ is $1.0 \mathrm{~s}^{-1}$ (Table 6.5).

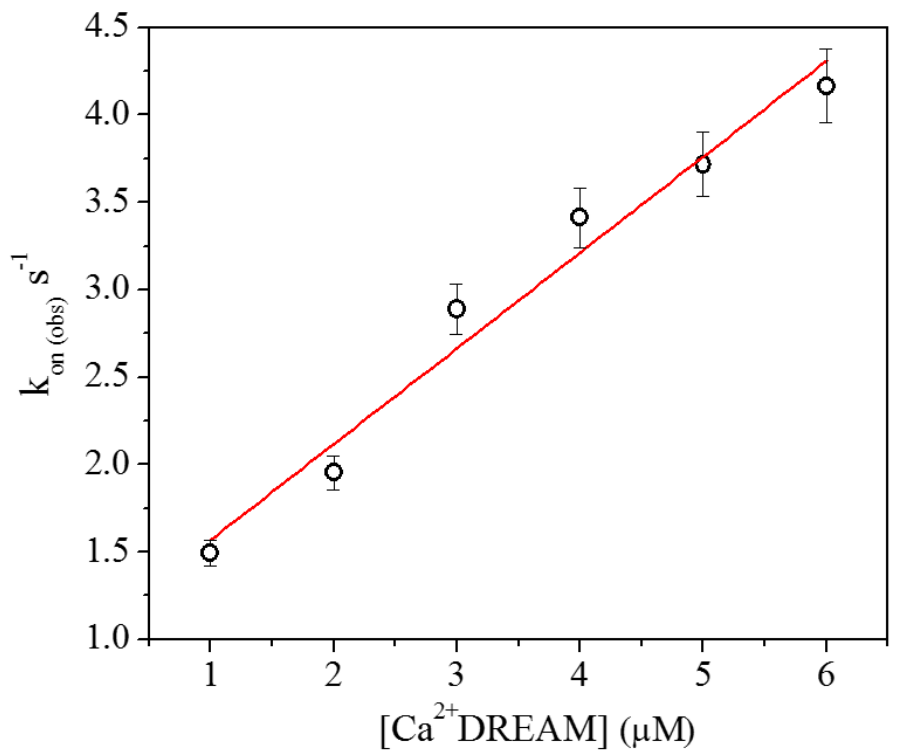

Figure 6.11: Apparent association rate constants $\left(\mathrm{k}_{\mathrm{on}(\mathrm{obs})}\right)$ were plotted against $\mathrm{Ca}^{2+}$ DREAM concentrations. The association rate constant $\left(\mathrm{k}_{\mathrm{on}}\right)$ and the dissociation rate constant $\left(\mathrm{k}_{\text {off }}\right)$ were determined to be is about $0.6 \times 10^{6} \mathrm{M}^{-1} \mathrm{~s}^{-1}$ and $1.0 \mathrm{~s}^{-1}$, respectively.

The association rate constant $\left(0.6 \times 10^{6} \mathrm{M}^{-1} \mathrm{~s}^{-1}\right)$ occurs within the range for bimolecular rate constants on the order of $10^{6} \mathrm{M}^{-1} \mathrm{~s}^{-1}$, which is about three-order in 
magnitude slower than the diffusion-limited association rate constant for a typical small spherical protein of $18 \AA$ radius $\left(7 \times 10^{9} \mathrm{M}^{-1} \mathrm{~s}^{-1}\right)$ (Northrup and Erickson, 1992). These results suggest that the association of HL9 with $\mathrm{Ca}^{2+}$ DREAM excludes diffusional components.

\subsubsection{Residue F252 plays an important role in regulating the interaction of DREAM with presenilin 1}

To test whether residue F252 in DREAM plays a crucial role in regulating the interaction of DREAM with PS1-HL9, titrations of $20 \mu \mathrm{M}$ DREAM with F252 replaced by A (herein named DREAM-F252A) into $0.5 \mu \mathrm{M}$ of PS1-HL9 in the presence and absence of $1 \mathrm{mM} \mathrm{Ca}^{2+}$ were conducted (Figure 6.12).

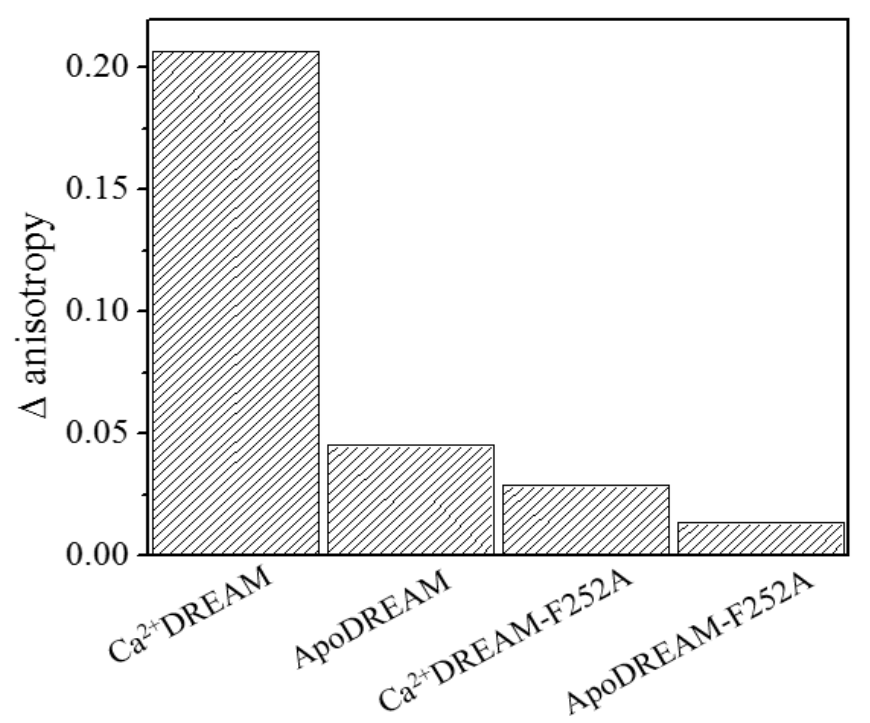

Figure 6.12: The bar plot represents titrations of $20 \mu \mathrm{M}$ of $\mathrm{Ca}^{2+}$ DREAM, ApoDREAM, $\mathrm{Ca}^{2+}$ DREAM-F252A, and ApoDREAM-F252A into 0.5 $\mu \mathrm{M}$ PS1-HL9.

The results show that replacement of residue F252 in DREAM by alanine significantly reduces anisotropy changes observed for PS1-HL9 association to either $\mathrm{Ca}^{2+}$ 
bound or free form of DREAM. These results confirm that F252 in DREAM plays an important role in regulating the interaction DREAM with PS-HL9.

Furthermore, the computational docking data reveal that the $\mathrm{Ca}^{2+}$ DREAM-PS1CTF complex is also stabilized by salt bridge interactions between residues R200 and R207 from the loop connecting $\alpha 7$ and $\alpha 8$ in DREAM and the residues D450 and D458 from HL9 of presenilin 1 (Figure 6.1). To test whether these salt bridge play an important role in modulating the interaction of DREAM with presenilin 1 , titration of $\mathrm{Ca}^{2+}$ DREAM into PS1-HL9 was conducted in buffer solution containing high concentration of salt $(0.3 \mathrm{M}$ $\mathrm{NaCl}$ ) (Figure 6.13). The titration data show that binding of $\mathrm{Ca}^{2+}$ DREAM to HL9 is salt independent with a $\mathrm{K}_{\mathrm{d}}$ similar to that observed in the absence of salt $(0.6 \mu \mathrm{M})$.

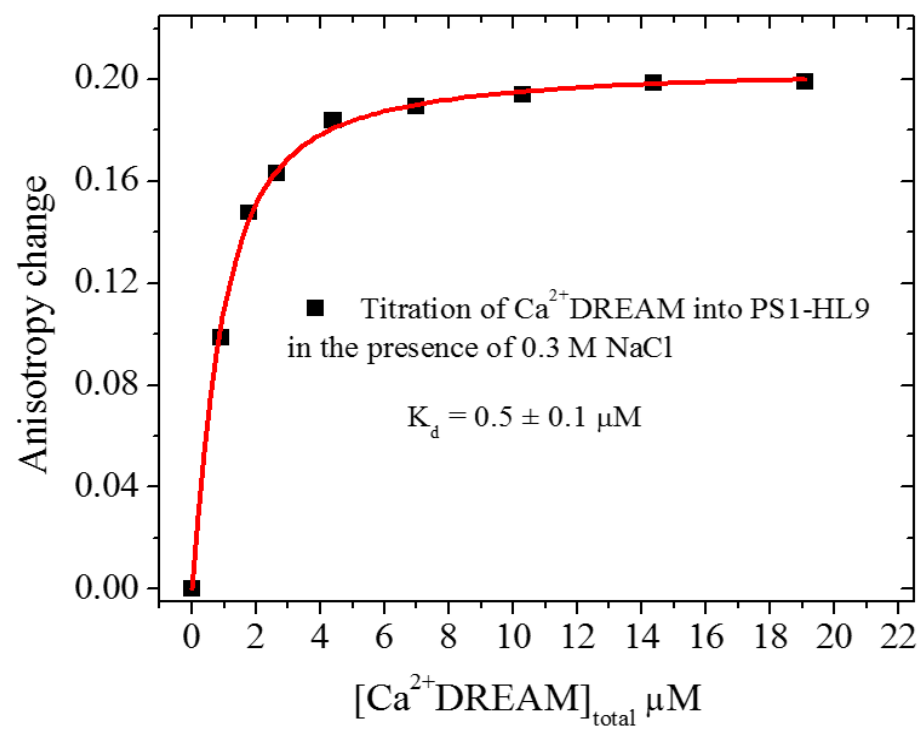

Figure 6.13: Titration curves for HL9 binding to $\mathrm{Ca}^{2+}$ DREAM in the presence of $0.3 \mathrm{M}$ $\mathrm{NaCl}$. The Solid line corresponds to the best fit using equation 3.63 in section 3.5.2. 


\subsubsection{Binding of PS1-CTF to DREAM inhibits the interaction of NS5806 drug compound with DREAM}

Previous studies in our laboratory demonstrated that binding of the drug compound NS5806 at the hydrophobic site on the C-terminus of DREAM increases the binding affinity between DREAM and the N-terminus of $\mathrm{K}^{+}$channel Kv4.3 (Gonzalez, Pham et al., 2014). The dissociation constant of NS5806 for $\mathrm{Ca}^{2+}$ DREAM was previously determined to be $2.5 \mu \mathrm{M}$ (Gonzalez, Pham et al., 2014). Interestingly, the residues Y174, F218, and F252 in DREAM (Figure 6.1) are responsible not only for the NS5806 drug binding but also for interaction with the HL9 in presenilin 1. Therefore, I speculated that the determined DREAM-presenilin interface hot spots (Figure 6.1) are druggable pockets for NS5806 compound that binds to DREAM and inhibits the interaction between DREAM and presenilin 1.

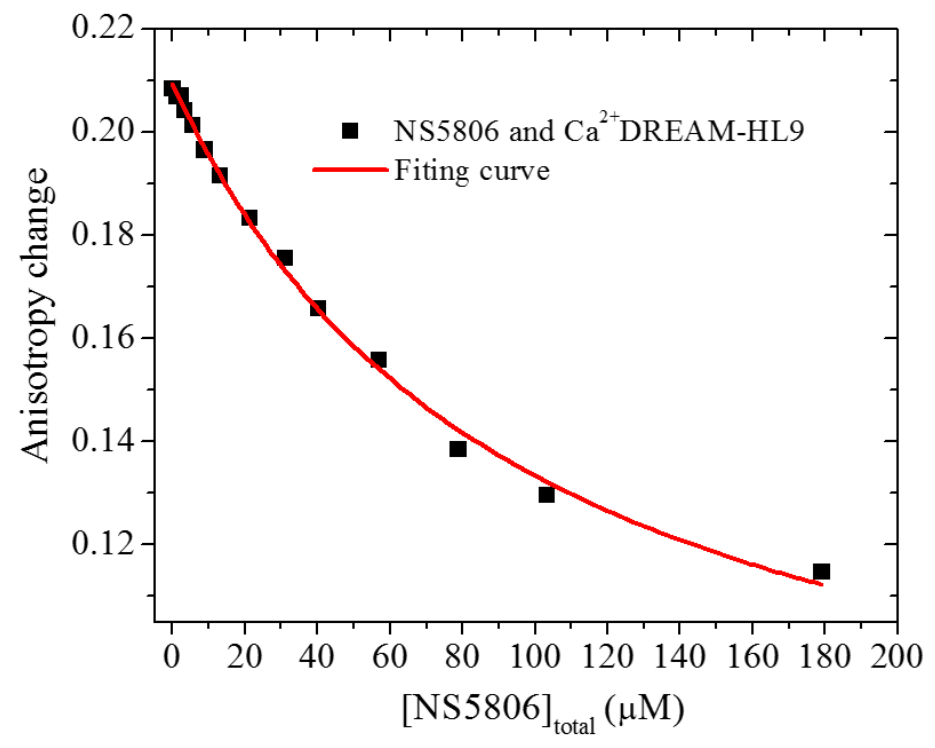

Figure 6.14: Titration of NS5806 into the $\mathrm{Ca}^{2+}$ DREAM-HL9 complex. The dissociation constant was determined by fitting the data using a single binding site displacement equation 3.64 in section 3.5.2. 
To test that hypothesis, the titration of NS5806 into the $\mathrm{Ca}^{2+}$ DREAM and HL9 complex was conducted (Figure 6.14). Titration data show that binding affinity of NS5806 to $\mathrm{Ca}^{2+}$ DREAM significantly decreases $\left(\mathrm{K}_{\mathrm{d}}=97 \mu \mathrm{M}\right)$ in the presence of PS1-HL9. The binding affinity of NS5806 for $\mathrm{Ca}^{2+}$ DREAM in the presence of PS1-HL9 significantly decreases compared to that determined in the absence of PS1-HL9 suggesting that binding of PS1-HL9 to DREAM blocks the docking site of NS5806 drug compound for DREAM at the hydrophobic residues Y174, F218, and F252 in DREAM.

\subsection{Discussion}

Previous studies have shown that DREAM and KChIP4 bind to the CTF of PS1 and PS2, however, the role of $\mathrm{Ca}^{2+} / \mathrm{Mg}^{2+}$ in regulating protein-protein interactions remains unclear. By using co-immunoprecipitation, Choi et al. have reported that the interaction of DREAM and PS2 is calcium independent (Choi, Zaidi et al. 2001), whereas Jo et al. have reported that DREAM association with PS1-CTF stimulates the activity of $\gamma$-secretase and facilitates production of $\mathrm{A} \beta 42$ plaques in Alzheimer's disease in the presence of $\mathrm{Ca}^{2+}(\mathrm{Jo}$, Jang et al. 2005b). Titration data show that $\mathrm{Ca}^{2+}$ DREAM binds to HL9 with high affinity $\left(\mathrm{K}_{\mathrm{d}}=0.6 \mu \mathrm{M}\right)$, whereas negligible binding was observed in the Apo form $\left(\mathrm{K}_{\mathrm{d}}=183 \mu \mathrm{M}\right)$. In addition, identical dissociation constants were determined for HL9 association HL9 with $\mathrm{Ca}^{2+}$ DREAM and $\mathrm{Ca}^{2+} \mathrm{Mg}^{2+}$ DREAM suggesting that $\mathrm{Ca}^{2+}$ but not $\mathrm{Mg}^{2+}$ functionally regulates interactions between DREAM and HL9. The titration data also demonstrate that DREAM does not interact with HL $\beta$ suggesting that the region in PS1 interfacing with DREAM comprises amino acid residues from HL9 (residues 445 - 467). These observations are consistent with previous studies demonstrating that the carboxy-terminal 
region in PS1 (residues 425 - 467) binds to DREAM and the homologous protein KChIP4 (Buxbaum, Choi et al. 1998, Morohashi, Hatano et al. 2002).

These results are in agreement with the model structure of $\mathrm{Ca}^{2+}$ DREAM:PS1-CTF determined by protein-protein docking. The assembly of $\mathrm{Ca}^{2+} \mathrm{DREAM}$ and PS1-CTF involves two interaction interfaces on DREAM protein. The first binding interface is formed by residues from the dynamic loop connecting $\alpha 7$ and $\alpha 8$ in DREAM, namely R207 which forms salt bridges with D450 and D458 from PS1 and R200 in DREAM which is involved in cation- $\pi$ interactions with F465 in PS1. The second interface is provided by hydrophobic residues located in the cavity between $\alpha 7$ and $\alpha 10$ of the DREAM carboxyterminal domain (Figure 6.1), namely residues M191, I194, M197, M198, M249, F252, V255, and I256 in DREAM which forms an aromatic cluster with F462 and F465 from PS1. The location of the binding sites in DREAM carboxy-terminal domain is confirmed by titration data between HL9 and DREAM-C. HL9 binds to DREAM-C with a $\mathrm{K}_{\mathrm{d}}$ that is 10 times lower than that determined for the full length DREAM. Since the NMR structure of the isolated DREAM-C in the presence of $\mathrm{Ca}^{2+}$ is nearly identical to the structure of the $\mathrm{Ca}^{2+}$ bound carboxy-terminal domain of the full length protein, the observed decrease in the DREAM-C affinity for HL9 reflects a distinct organization of individual monomers within the DREAM-C dimer due to the lack of amino-terminal domain in DREAM-C compared to DREAM (Yu et al., 2007; Lusin et al., 2008).

To understand the mechanism of how $\mathrm{Ca}^{2+}$ regulates DREAM and PS1-CTF interactions, the structure of $\mathrm{Ca}^{2+}$ DREAM was superposed with the model structure of ApoDREAM (Pham et al., 2015) as shown in Figure 6.15. In the ApoDREAM structure, the aromatic ring of F252 rotates $\sim 90$ degrees preventing $\pi-\pi$ stacking with aromatic rings 
of F462 and F465 from PS1 and destabilizing the aromatic cluster between phenylalanine residues from DREAM and PS1-CTF. In addition, the guanidinium group of R200 points away from the center of aromatic ring of F465 in PS1, weakening the interactions between $\alpha 7 / \alpha 8$ loop and PS1. Also in the $\mathrm{Ca}^{2+}$ bound DREAM, $\alpha 10$ moves away from $\alpha 7$, enhancing the accessibility of the hydrophobic cavity between $\alpha 7$ and $\alpha 10$ in $\mathrm{Ca}^{2+}$ DREAM.

A high activation energy observed for HL9 association with $\mathrm{Ca}^{2+}$ DREAM, likely reflects a conformational changes in the DREAM carboxyl domain during HL9 binding. This structural rearrangement at the binding interfaces may include the repositioning of $\alpha 10$ away from $\alpha 7$ as well as a reduction in the flexibility of $\alpha 7 / \alpha 8$ loop of DREAM. Increased accessibility of the carboxyl cavity in the $\mathrm{Ca}^{2+}$ bound DREAM was previously observed using hydrophobic fluorescent probes and associated with the increased DREAM affinity for arachidonic acid and site one of $\mathrm{K}_{\mathrm{v}}$ channel (Gonzalez, Pham et al., 2014).
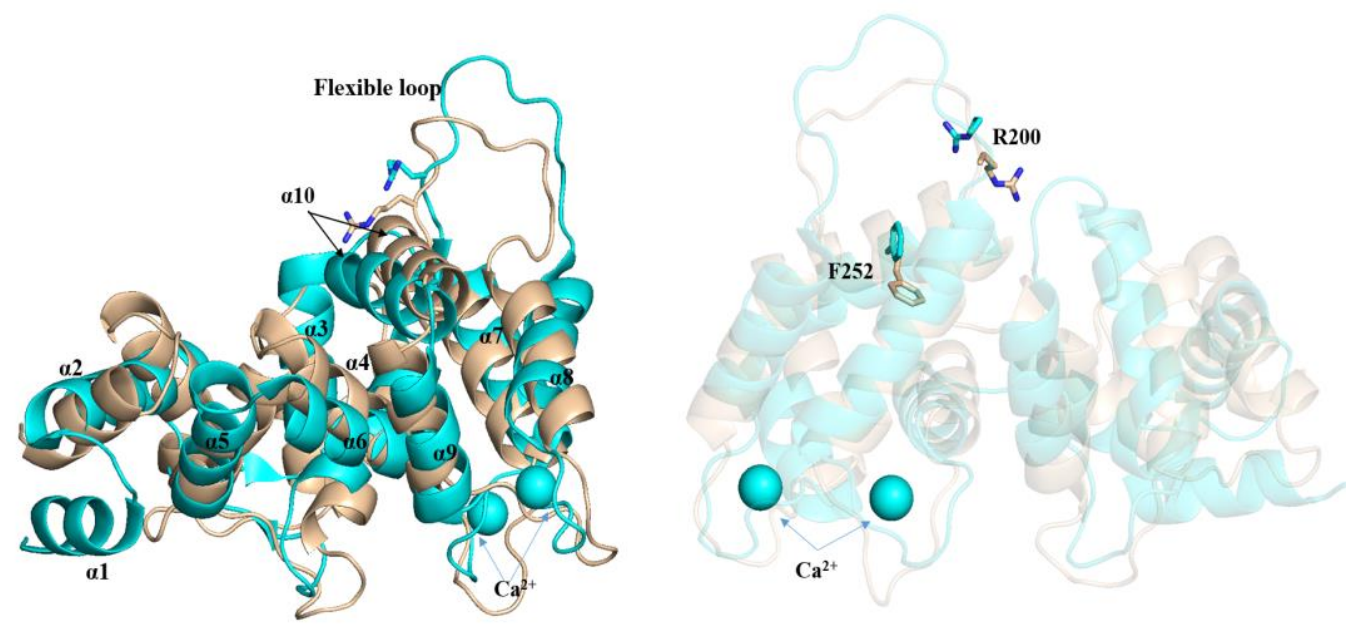

Figure 6.15: Left: Superposed structures of $\mathrm{Ca}^{2+}$ DREAM (PDB code: $2 \mathrm{JUL}$, molecule 1, cyan) and molecular dynamics simulation structure of ApoDREAM (yellow). Alpha helices $1-10$ in DREAM are labeled by $\alpha 1-\alpha 10$, respectively. The movement of $\alpha 10$ away from $\alpha 7$ is indicated by black arrows. Right: aromatic ring of F252 in ApoDREAM rotates $\sim 90$ degrees with respect to aromatic ring of F252 in $\mathrm{Ca}^{2+}$ bound DREAM. The guanidinium group of R200 in ApoDREAM points 180 degrees away with respect to that of $\mathrm{Ca}^{2+}$ DREAM. 
The relevance of the flexible loop in DREAM to association of HL9 is further highlighted by titration of HL9 with $\mathrm{Ca}^{2+}$ DREAM-NCS1 chimera construct. These results show that binding affinity for HL9 to $\mathrm{Ca}^{2+}$ DREAM-NCS1 is decreased $\left(\mathrm{K}_{\mathrm{d}}=38 \mu \mathrm{M}, \Delta \mathrm{G}\right.$ $\left.=-5.9 \mathrm{kcal} \mathrm{mol}^{-1}\right)$ compared to that of DREAM $\left(\mathrm{K}_{\mathrm{d}}=0.6 \mu \mathrm{M}, \Delta \mathrm{G}=-8.3 \mathrm{kcal} \mathrm{mol}^{-1}\right)$. The difference in binding affinity is expressed in terms of difference in the free energy $(\Delta \Delta \mathrm{G})$ of $-2.4 \mathrm{kcal} \mathrm{mol}^{-1}$ which is comparable to the formation energy of a salt bridge. The binding affinity of HL9 to $\mathrm{Ca}^{2+}$ DREAM-KChIP1 chimera is only partially reduced $\left(\mathrm{K}_{\mathrm{d}}=3.9 \mu \mathrm{M}\right.$, $\left.\Delta \mathrm{G}=-7.2 \mathrm{kcal} \mathrm{mol}^{-1}\right)$. Amino acid sequence alignment of mouse DREAM with various KChIP members and NCS1 protein shows that the hydrophobic residues in the DREAM carboxyl terminus are well conserved and thus selectivity for PS1/PS2 is modulated by residues located in the $\alpha 7 / \alpha 8$ loop (Figure 6.4). For instance, the substitution of basic residues R200 and R207 in DREAM by a neutral residue N200 and an acidic residue E207 from NCS1 is likely responsible for reducing the binding affinity of DREAM-NCS1 chimera and HL9. Also, a replacement of R200 in DREAM by K200 from KChIP1 may contribute to the lower binding affinity of DREAM-KChIP1 chimera and HL9 due to a weaker cation-pi interaction between Phe and Lys compared to that of Phe and Arg (Andrew et al., 2002). However, addition of $300 \mathrm{mM} \mathrm{NaCl}$ does not affect binding affinity of $\mathrm{HL} 9$ and $\mathrm{Ca}^{2+}$ DREAM (Figure 6.13) suggesting that interactions between $\mathrm{Ca}^{2+}$ DREAM and HL9 comprise predominantly van der Waals contacts among hydrophobic residues exemplified by interactions of F462 and F465 residues from HL9 with F252 in $\alpha 10$ in DREAM as well as the residues at the hydrophobic crevice near $\alpha 10$. 
Moreover, binding of PS1-CTF to DREAM inhibits interaction of the NS5806 drug compound with DREAM. Furthermore, the highly conserved amino acid residues at the region of HL9 in both PS1 and PS2 suggest that DREAM may also interact with PS2 with similar binding interfaces as determined in the DREAM and PS1 complex.

\subsection{Summary}

The results presented here provide insight into the nature of binding interfaces between DREAM and HL9 peptide and demonstrate the role of $\mathrm{Ca}^{2+} / \mathrm{Mg}^{2+}$ in DREAM:HL9 complex formation. The hydrophobic cavity in the DREAM carboxyterminal domain provide the binding site for HL-9 from presenilin and the complex is stabilized through interactions between the dynamic loop between helix 7 and 8 in DREAM and HL9 residues. The identified DREAM-presenilin interface hot spots provide druggable pockets for structure-based development of a novel drug compound that binds to DREAM and inhibits the interaction of DREAM with presenilin as an efficient treatment of Alzheimer's disease. 


\section{THE ROLE OF INDIVIDUAL EF-HANDS IN MODULATING DREAM CONFORMATIONAL DYNAMICS AND INTERACTION WITH DNA}

(The results in this chapter were described in a manuscript that will be submitted to the Biochemistry journal by Pham and Miksovska)

\subsection{Background and significance}

Downstream regulatory element antagonist modulator, also known as $\mathrm{K}^{+}$channel interacting protein 3 (KChIP3) or calsenilin, is a neuronal calcium sensor (NCS) protein that acts as a transcriptional repressor of prodynorphin/c-fos gene to modulate pain signaling in the spinal cord (Buxbaum et al., 1998; Carrion et al., 1999; An et al., 2000; Cheng et al., 2002). Structurally, DREAM contains four EF-hand $\mathrm{Ca}^{2+}$ binding motifs, of which EF-hand 1 does not bind $\mathrm{Ca}^{2+} / \mathrm{Mg}^{2+}$ due to the presence of conserved residues $\mathrm{C} 104$ and P105 on the first EF-hand (Flaherty et al., 1993). In addition, EF-hand 2 selectively binds $\mathrm{Mg}^{2+}$ with a high affinity $\left(\mathrm{K}_{\mathrm{d}} \sim 13 \mu \mathrm{M}\right)$, whereas EF-hands 3 and 4 exclusively bind to $\mathrm{Ca}^{2+}$ with a relatively high affinity $\left(\mathrm{K}_{\mathrm{d}} \sim 1 \mu \mathrm{M}\right)$ (Osawa et al., 2005). Functionally, interactions of DREAM with intracellular target proteins and DNA are either $\mathrm{Ca}^{2+}$ dependent or independent. These interactions are involved in numerous physiological functions, including prodynorphin/c-fos gene transcriptional repression (Carrion et al., 1999; Osawa et al., 2001), potassium channel activity modulation (An et al., 2000), enhancement of calcineurin activation by binding to calmodulin (Ramachandran et al., 2012), modulation of presenilins enzymatic activity (Buxbaum et al., 1998; Buxbaum, 2004, Pham and Miksovska 2016), neuronal apoptosis, and $\mathrm{Ca}^{2+}$ signaling in the endoplasmic recticulum (Buxbaum et al., 1998; Leissring et al., 2000; Jo et al., 2001; 
Lilliehook et al., 2002; Jo et al., 2004; Jo et al., 2005; Fedrizzi et al., 2008; Jang et al., 2011). The mechanism of pain processing by transcriptional repression of prodynorphin/cfos gene has gained much attention in recent decades (Carrion et al., 1999; Osawa et al., 2001; Cheng et al., 2002, Costigan and Woolf, 2002; Osawa et al., 2005; Lusin et al, 2008). However, a detailed insight into the molecular mechanism of DREAM interaction with downstream regulatory elements (herein named DNA) of prodynorphin/c-fos gene, the functional role of individual EF-hands in regulating the DREAM conformational dynamics and oligomerization of the DREAM-DNA complex formation remain unclear.

The critical barrier for envisioning the molecular mechanism of interaction between DREAM and DNA lies in unknown structures of the Apo and $\mathrm{Mg}^{2+}$ DREAM. The primary goal of this study was to delineate the structure-dynamics-function relationship of DREAM in modulating the interaction with DNA. To accomplish this goal, the impact of $\mathrm{Mg}^{2+}$ and/or $\mathrm{Ca}^{2+}$ on the conformational dynamics of DREAM and functional EF-hands 2, 3, and 4 were characterized in order to highlight the role of individual EF-hands. The interaction between DREAM and DNA in the $\mathrm{Ca}^{2+}$ and/or $\mathrm{Mg}^{2+}$ dependent was also identified by characterizing the association of DNA with DREAM and DREAM EF-hand mutants. The binding interface of the ApoDREAM-DNA complex, as well as the conformational dynamics of ApoDREAM upon DNA association, were also examined using computational approaches. Recently, advances in computational chemistry, including computational docking (Comeau et al. 2004; Comeau et al., 2004; van Dijk et al., 2006; de Vries et al., 2010; Lyskov and Gray, 2008; van Dijk and Bonvin, 2009), molecular dynamics simulations (Brooks et al., 1983), and protein dynamical network analysis (Sethi et al., 2009), have opened the opportunity not only to visualize the binding interface 
between DREAM and DNA, but also to observe the dynamic structures of DREAM in the Apo, $\mathrm{Mg}^{2+}$, and $\mathrm{Ca}^{2+}$ bound forms at the level of individual amino acid residue. In addition, distinct signaling pathways, propagated by $\mathrm{Ca}^{2+}$ and $\mathrm{Mg}^{2+}$ from their binding sites to the DNA binding interface. The results of computational studies were integrated with experimental information to elucidate the structure-function relationship of DREAM in modulating the interaction with DNA.

I employed isothermal titration calorimetry (ITC) to investigate the association of DNA to DREAM and DREAM EF-hand mutants in terms of binding affinity $\left(\mathrm{K}_{\mathrm{d}}\right)$, binding stoichiometry $(\mathrm{n})$, and binding energy $(\Delta \mathrm{H})$. Additionally, the oligomeric states of DREAM in complex with DNA were characterized using fluorescence anisotropy decays. In parallel, the roles of individual EF-hands 2, 3, and 4 in DREAM were also investigated by analyzing fluorescence emission and lifetimes of the intrinsic tryptophan residue (W169) in DREAM EF-hand mutants. Lastly, the binding interfaces between ApoDREAM and DNA were determined using a combination of computational docking (de Vries et al., 2010), molecular dynamics (MD) simulation (Phillips et al., 2005), and dynamical network analysis (Sethi et al., 2009; Alexander et al., 2010).

\subsection{Results}

\subsubsection{Steady-state emission spectra of tryptophan.}

The single tryptophan residue (W169) in DREAM, located at the interface between the $\mathrm{N}$ - and C-terminal domains, is an ideal fluorescence probe for investigation of the protein conformational dynamics of DREAM EF-hand mutants upon binding to $\mathrm{Ca}^{2+}$ and/or $\mathrm{Mg}^{2+}$ (Pham et al., 2015). The approach enables us to elucidate the functional role of each EF-hands in DREAM. The emission spectra of DREAM EF-hand 2 mutant 
(DREAM-D150N) are almost identical in the Apo and $\mathrm{Mg}^{2+}$ forms, with the emission maximum centered at $340 \mathrm{~nm}$ (Figure 7.1, left panel). $\mathrm{Ca}^{2+}$ association at EF-hands 3 and 4 in DREAM-D150N mutant leads to a decrease in emission intensity along with $5 \mathrm{~nm}$ blue-shift of emission maximum in the $\mathrm{Ca}^{2+}$ DREAM emission spectrum compared to that obtained in the Apo or $\mathrm{Mg}^{2+}$ form (Figure 7.1, left panel).
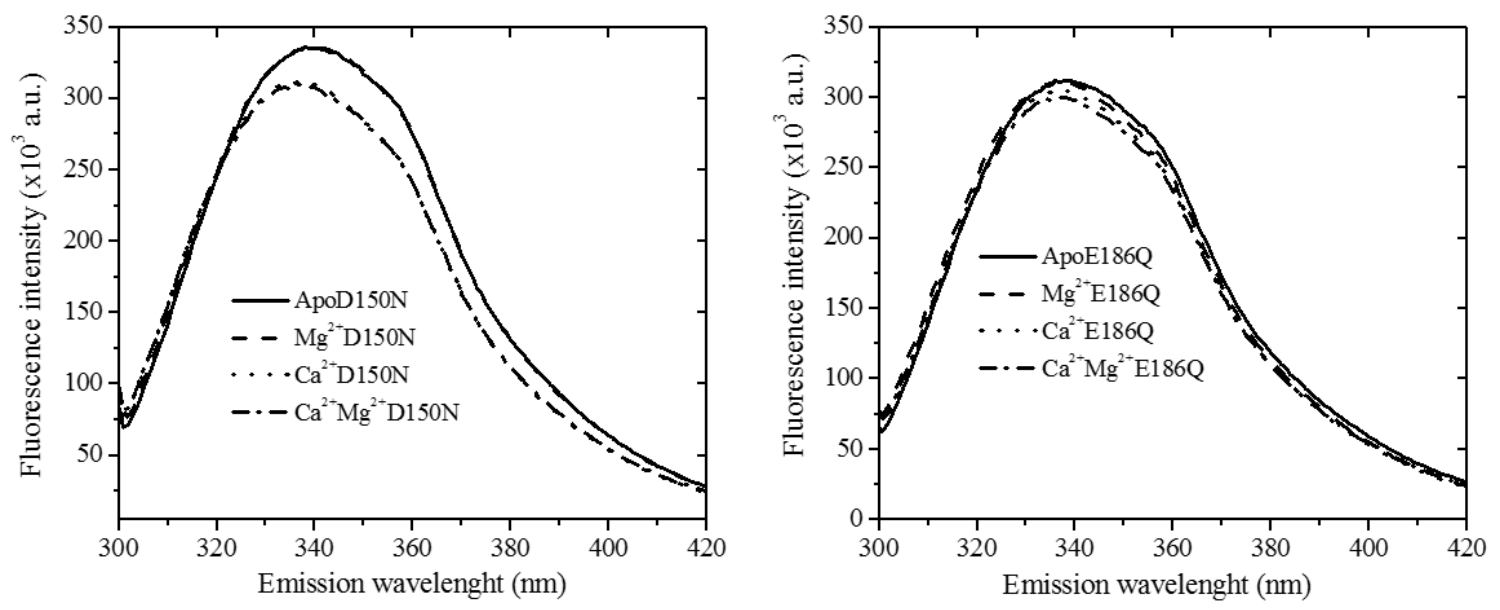

Figure 7.1: Tryptophan (W169) emission spectra of Apo, $\mathrm{Mg}^{2+}, \mathrm{Ca}^{2+}$, and $\mathrm{Ca}^{2+} \mathrm{Mg}^{2+}$ bound DREAM-D150N (left panel) and DREAM-E186Q (right panel) upon excitation at $295 \mathrm{~nm}$. Conditions: $40 \mu \mathrm{M}$ DREAM in $20 \mathrm{mM}$ Tris $\mathrm{pH}$ 7.4, $10 \mathrm{mM}$ LDAO, and $1 \mathrm{mM}$ DTT.

These results suggest that $\mathrm{Ca}^{2+}$ association to DREAM-D150N propagates structural rearrangement, by which W169 residue becomes more buried in a hydrophobic environment in a similar way as observed previously for DREAM wild-type (DREAMWT) described in chapter 4 (Pham et al., 2015). However, a small but reproducible deviation was observed in the emission spectra of DREAM-D150N relative to that of DREAM-WT. Upon $\mathrm{Ca}^{2+}$ association, emission intensity of $\mathrm{Ca}^{2+}$ DREAM decreases about $18 \%$ relative to that of ApoDREAM as previously described in section 4.2.1 (Pham et al., 2015). On the other hand, the emission intensity of $\mathrm{Ca}^{2+}$ DREAM-D150N decreases about 
$11 \%$ compared to that of ApoDREAM-D150N. These results suggest a small structural difference in either the Apo or $\mathrm{Ca}^{2+}$ bound form of DREAM-D150N compared to DREAM, but overall the mutation D150 $\rightarrow \mathrm{N}$ at EF-hand 2 in DREAM does not affect the conformational transition triggered by $\mathrm{Ca}^{2+}$ in the protein.

The association of $\mathrm{Ca}^{2+}$ to DREAM EF-hand 3 mutant (DREAM-E186Q) leads to a small but reproducible decrease in emission intensity ( $2 \%)$ along with a $2 \mathrm{~nm}$ blue-shift of emission maximum in $\mathrm{Ca}^{2+}$ DREAM-E186Q emission spectrum relative to that observed in the ApoDREAM-E186Q (Figure 7.1, right panel). These results illustrate that $\mathrm{Ca}^{2+}$ association to EF-hand 4 in DREAM-E186Q does not trigger a significant change in the protein tertiary structure, suggesting that the side chain of W169 residue remains solvent exposed in the $\mathrm{Ca}^{2+}$ bound form.

On the other hand, $\mathrm{Ca}^{2+}$ association to DREAM EF-hand 4 mutant (DREAME234Q) results in about $11 \%$ decrease in the emission intensity along with $5 \mathrm{~nm}$ blue-shift of emission maximum on $\mathrm{Ca}^{2+}$ DREAM-E234Q emission spectrum relative to that ApoDREAM-E234Q (Figure 7.2). These changes in emission intensity and hypsochromic shift are similar to that obtained in DREAM-D150N mutant and DREAM wild-type. These results indicate that binding of $\mathrm{Ca}^{2+}$ at EF-hand 3 induces structural transition in DREAME234Q mutant. In contrast, association of $\mathrm{Mg}^{2+}$ to ApoDREAM-E234Q does not lead to changes in W169 surrounding as evident from the emission spectrum of ApoDREAME234Q is similar to that of $\mathrm{Mg}^{2+}$ DREAM-E234Q. Interestingly, emission intensity of $\mathrm{Ca}^{2+} \mathrm{Mg}^{2+}$ DREAM-E234Q is increased about $5 \%$ relative to that of the $\mathrm{Ca}^{2+}$ DREAME234Q. 


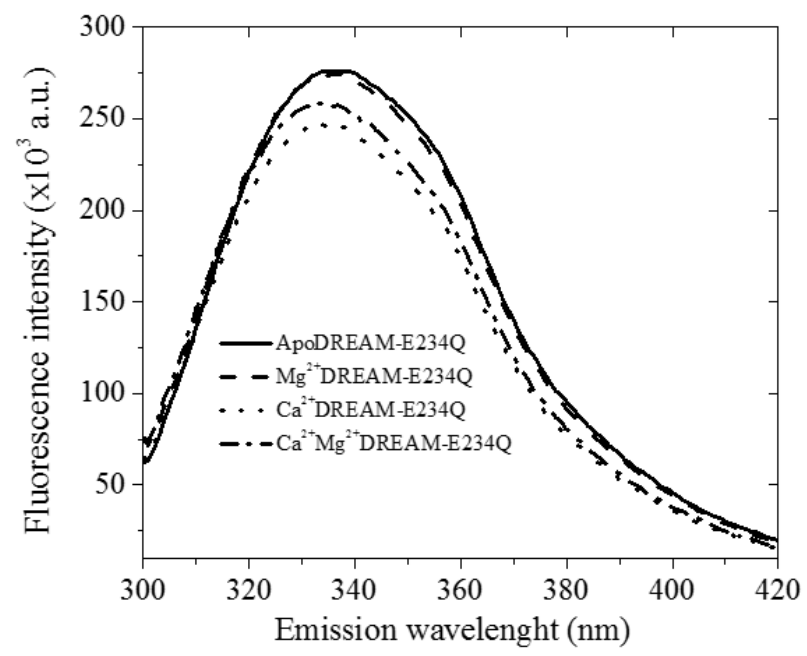

Figure 7.2: W196 emission spectra of Apo, $\mathrm{Mg}^{2+}, \mathrm{Ca}^{2+}$, and $\mathrm{Ca}^{2+} \mathrm{Mg}^{2+}$ bound DREAME234Q. Conditions: $40 \mu \mathrm{M}$ DREAM in $20 \mathrm{mM}$ Tris $\mathrm{pH} 7.4,10 \mathrm{mM}$ LDAO, and $1 \mathrm{mM}$ DTT.

These results suggest that the replacement of E234 by Q leads an increase the binding affinity for $\mathrm{Mg}^{2+}$ at EF-hand 4 with $\mathrm{Mg}^{2+}$ binding to EF-hand 4 impacts the emission of W169. Also, we cannot exclude the mutation of EF-hand 4 impacts the emission properties of $\mathrm{W} 169$ in the $\mathrm{Mg}^{2+}$ bound DREAM.

\subsubsection{Association of $\mathrm{Ca}^{2+}$ triggers changes in hydrophobicity at the surface of DREAM-EF hand mutants}

Previous work in our lab demonstrated that a large hydrophobic pocket exists on the surface of DREAM wild-type (Gonzalez and Miksovska, 2014). As the apolar cavity may provide a binding site for small hydrophobic molecules, surface hydrophobicity of DREAM was further explored. DREAM EF-hand mutants and their $\mathrm{Ca}^{2+}$ and/or $\mathrm{Mg}^{2+}$ bound forms were studied using steady-state fluorescence spectroscopy. Extrinsic fluorophores 8-anilino 1-naphathalene sulfonate (1,8-ANS) and 6-anilino 2-naphthalene 
sulfonate (2,6-ANS) were employed to probe the surface hydrophobicity due to their high sensitivity to their local environment.

The increase in emission intensity of 1,8-ANS in the presence of DREAM-D150N indicates that ANS association to the protein is independent of deactivated EF-hand 2. Significant increase in emission intensity was observed upon $\mathrm{Ca}^{2+}$ association to both Apo and $\mathrm{Mg}^{2+}$ DREAM-D150N (Figure 7.3, left panel). Interestingly, the emission spectra of 1,8-ANS bound to $\mathrm{Ca}^{2+}$ - and $\mathrm{Ca}^{2+} \mathrm{Mg}^{2+}$ DREAM-D150N are similar suggesting that the change in surface hydrophobicity is dependent solely on $\mathrm{Ca}^{2+}$ association. Moreover, previous studies have shown that $\mathrm{Mg}^{2+}$ binding to DREAM wild-type leads to a small but reproducible increase in the 1,8-ANS emission compared to that of Apo form (Gonzalez and Miksovska, 2014). In contrast, association of $\mathrm{Mg}^{2+}$ to ApoDREAM-D150N does not change in 1,8-ANS emission intensity relative to that of Apo form suggesting that the replacement of D150 by $\mathrm{N}$ abolishes the association of $\mathrm{Mg}^{2+}$ at EF-hand 2.
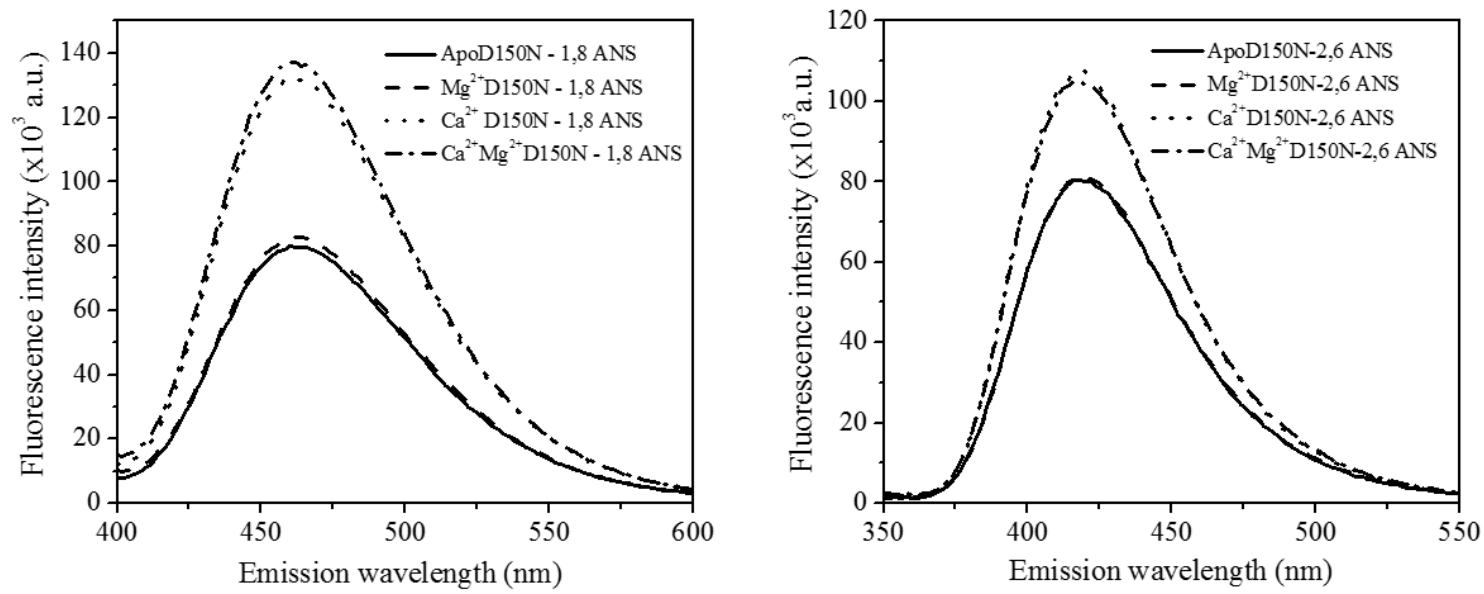

Figure 7.3: Fluorescence emission spectra of 1,8-ANS (left panel) and 2,6-ANS (right panel) bound to DREAM-D150N $(40 \mu \mathrm{M})$. The excitation wavelength was $350 \mathrm{~nm}$ and $319 \mathrm{~nm}$ for 1,8-ANS and 2,6-ANS, respectively. 
The results for 2,6-ANS bound to DREAM-D150N are consistent with those obtained using 1,8-ANS probe (Figure 7.4, right panel). A significant increase in the emission intensity of 2,6-ANS upon $\mathrm{Ca}^{2+}$ association to both Apo and $\mathrm{Mg}^{2+}$ DREAMD150N was observed. Likewise, the emission intensity of 2,6-ANS is remained unchanged in both Apo and $\mathrm{Mg}^{2+}$ forms.

Furthermore, the same fluorescence emission pattern was observed in EF-hand 3 mutant. Emission intensity change obtained for either 1,8-ANS or 2,6-ANS bound to DREAM-E186Q upon $\mathrm{Ca}^{2+}$ association to both the Apo and $\mathrm{Mg}^{2+}$ forms was comparable to the aforementioned results for DREAM-D150N (Figure 7.4, left and right panels, respectively). Likewise, identical change in emission intensity was also observed for either 1,8- or 2,6-ANS bound to the Apo and $\mathrm{Mg}^{2+}$ forms of DREAM-E186Q.
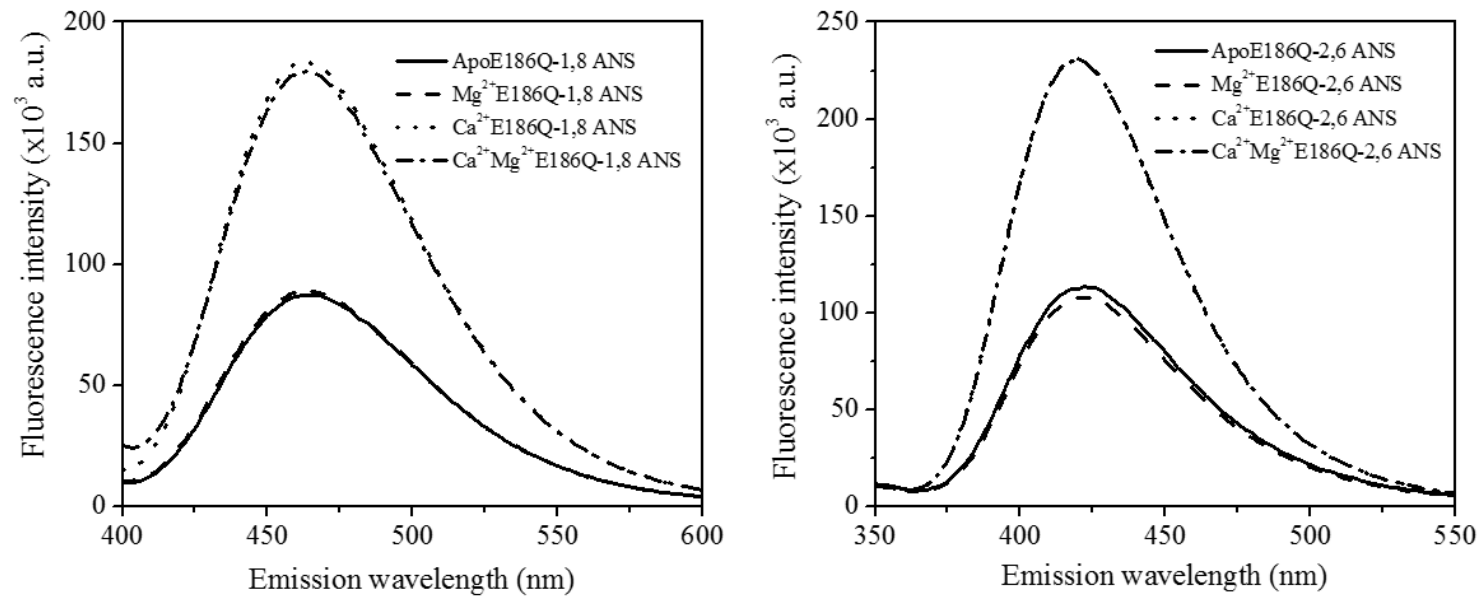

Figure 7.4: Fluorescence emission spectra of 1,8-ANS (left panel) and 2,6-ANS (right panel) bound to DREAM-E186Q $(40 \mu \mathrm{M})$. The excitation wavelength was $350 \mathrm{~nm}$ and $319 \mathrm{~nm}$ for 1,8-ANS and 2,6-ANS, respectively.

Furthermore, the identical results were also observed in the emission intensity of both 1,8- and 2,6-ANS in the presence of Apo and $\mathrm{Mg}^{2+}$ DREAM-E234Q (Figure 7.5 left and right panels). A further increase in intensity was obtained upon $\mathrm{Ca}^{2+}$ association to 
both the Apo and $\mathrm{Mg}^{2+}$ forms. However, association of $\mathrm{Mg}^{2+}$ to ApoDREAM-E234Q leads to a small but reproducible increase in the 1,8- and 2,6-ANS emission intensity relative to that of the Apo form. These results suggest that $\mathrm{Mg}^{2+}$ binding to EF-hand 4 may impact the emission of ANS, or the mutation of EF-hand 4 may affect the emission properties of ANS in the $\mathrm{Mg}^{2+}$ bound form.
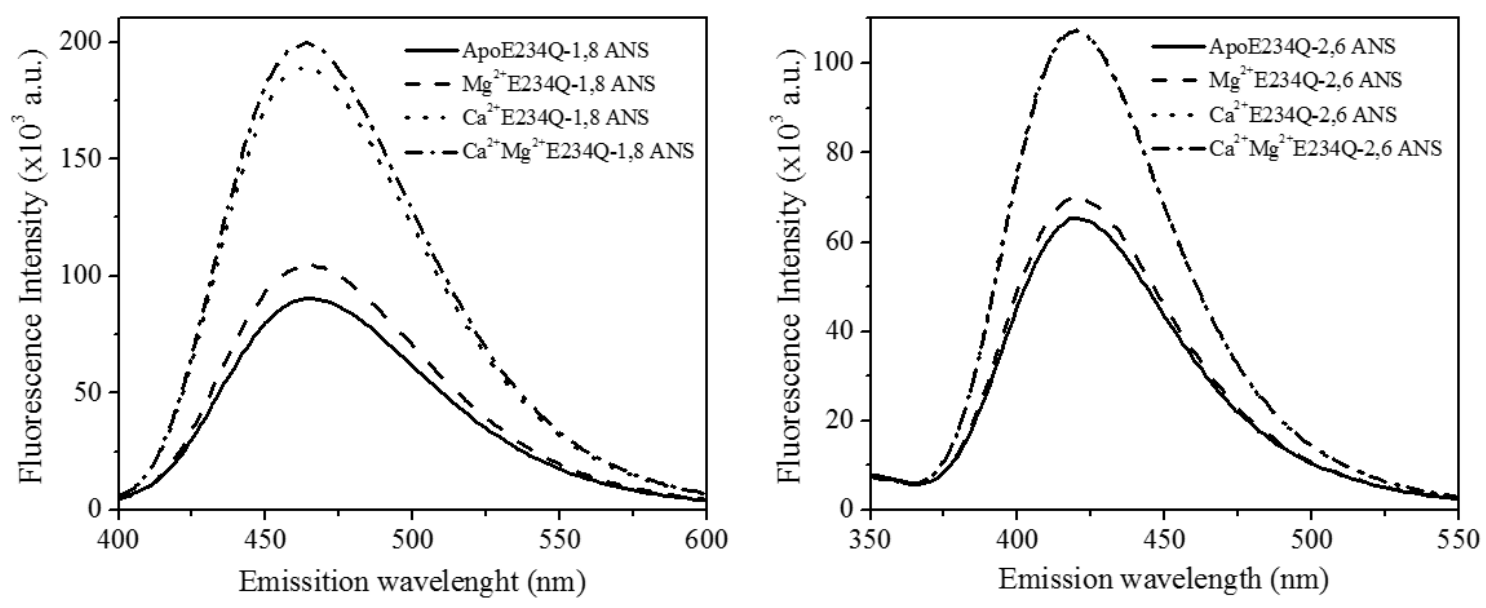

Figure 7.5: Fluorescence emission spectra of 1,8-ANS (left panel) and 2,6-ANS (right panel) bound to DREAM-E234Q $(40 \mu \mathrm{M})$. The excitation wavelength was $350 \mathrm{~nm}$ and $319 \mathrm{~nm}$ for 1,8-ANS and 2,6-ANS, respectively.

\subsubsection{Fluorescence intensity decay of tryptophan}

Since the tryptophan fluorescence lifetime is highly sensitive to small variations in the fluorophore environment, probing the lifetime of W169 in DREAM provides further insight into $\mathrm{Ca}^{2+}$ and/or $\mathrm{Mg}^{2+}$ induced changes in protein dynamics. The W169 lifetime data of DREAM mutants were best fit using a combination of Gaussian distribution and discrete exponential model. Goodness of the fits was judged based on residuals and the $\chi^{2}$ value of the non-linear least square fitting curve. The W169 lifetime parameters of DREAM EF-hand mutants are summarized in Table 7.1. 
The DREAM-D150N (Figure 7.6, left panel) exhibits two distinct lifetimes, of which the discrete lifetime component $\left(\tau_{2}\right)$ of $7.4 \mathrm{~ns}$ is $\mathrm{Ca}^{2+}$ independent, whereas Gaussian distribution lifetime component $\left(\tau_{1}\right)$ varies upon $\mathrm{Ca}^{2+}$ binding to the $\mathrm{Apo}$ or $\mathrm{Mg}^{2+}$ forms. In the Apo form, the components $\tau_{1}$ is centered at $2.9 \mathrm{~ns}$ with a distribution width $\left(\mathrm{w}_{1}\right)$ of 1.3 ns. Upon $\mathrm{Ca}^{2+}$ association, these values of $\tau_{1}$ and $\mathrm{w}_{1}$ decrease to $1.9 \mathrm{~ns}$ and $0.7 \mathrm{~ns}$, respectively. In addition, the fractional contribution of the Gaussian distribution $\left(f_{1}\right)$ also decreases from $39 \%$ in the Apo to $16 \%$ for the $\mathrm{Ca}^{2+}$ bound form. These results are similar to the results determined for DREAM-WT (see Chapter 4).
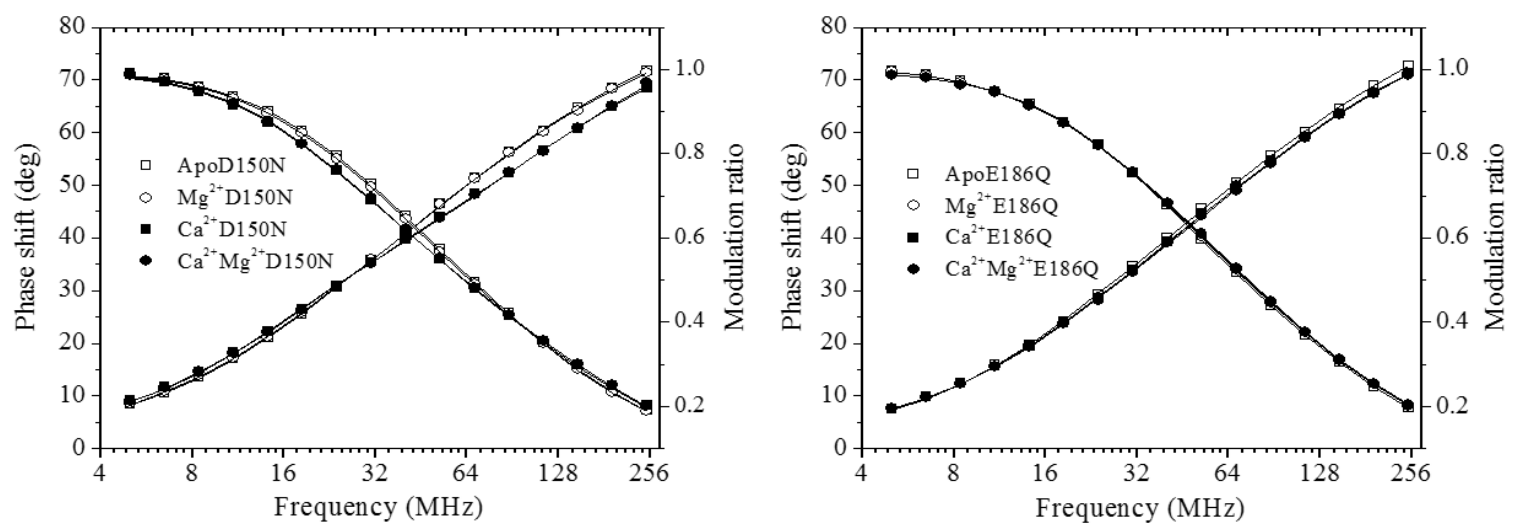

Figure 7.6: Frequency domain W169 intensity decay of $\mathrm{Apo}, \mathrm{Mg}^{2+}, \mathrm{Ca}^{2+}$, and $\mathrm{Ca}^{2+} \mathrm{Mg}^{2+}$ bound DREAM-D150N (left panel) and DREAM-E186 (right panel). The solid lines represent the data fitting using a combination of Gaussian distribution and discrete exponential decay model. The recovered parameters are shown Table 7.1.

In contrast, $\mathrm{Ca}^{2+}$ association at EF-hand 4 in DREAM-E186Q (Figure 7.6, right panel) does not induce a structural change in the protein as evident from constant lifetime components $\tau_{1}$ of $2.1 \mathrm{~ns}$ and $\tau_{2}$ of $5.9 \mathrm{~ns}$ in both Apo and $\mathrm{Ca}^{2+}$ and/or $\mathrm{Mg}^{2+}$ bound forms. In addition, the Gaussian distribution width $\left(\mathrm{w}_{1}\right)$ and the fractional contribution $\left(f_{1}\right)$ parameters are almost identical in the $\mathrm{Ca}^{2+}$ free and $\mathrm{Ca}^{2+}$ bound forms of the protein. 
On the other hand, $\mathrm{Ca}^{2+}$ association at EF-hand 3 in DREAM-E234Q (Figure 7.7) induces similar change in protein dynamics as observed for DREAM-WT and DREAMD150N mutant. Namely, $\mathrm{Ca}^{2+}$ association leads to a decrease in W169 lifetime component $\tau_{1}$ from $2.5 \mathrm{~ns}$ in the Apo to $1.8 \mathrm{~ns}$ for the $\mathrm{Ca}^{2+}$ bound form.

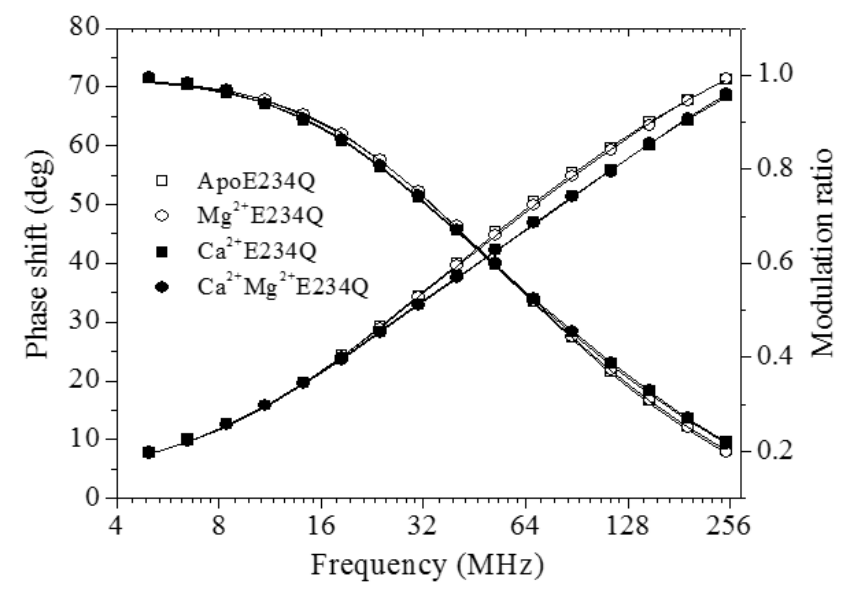

Figure 7.7: Frequency domain intensity decay of Apo, $\mathrm{Mg}^{2+}, \mathrm{Ca}^{2+}$, and $\mathrm{Ca}^{2+} \mathrm{Mg}^{2+}$ bound DREAM-E234Q $(40 \mu \mathrm{M})$. The solid lines represent the data fitting using a combination of Gaussian distribution and discrete exponential decay model

Moreover, the Gaussian distribution width $\left(\mathrm{w}_{1}\right)$ becomes narrower in the $\mathrm{Ca}^{2+}$ bound form ( $0.76 \mathrm{~ns})$ compared to that obtained for the Apo or $\mathrm{Mg}^{2+}$ form (1.1 ns). Similar to other DREAM constructs, the W169 lifetime component $\tau_{2}$ of DREAM-E234Q (6.3 ns) is independent of $\mathrm{Ca}^{2+}$ association. Our fluorescence lifetime results suggest that $\mathrm{Ca}^{2+}$ association at EF-hand 3 but not EF-hand 4 triggers significant alteration in the tertiary structure and/or modulates conformational flexibility of DREAM protein. These results are in accordance with that obtained in the steady-state emission study. 
Table 7.1: Emission decay parameters of W169 in DREAM EF-hand mutants using a continuous Gaussian distribution and a discrete component.

\begin{tabular}{|c|c|c|c|c|c|c|c|c|c|}
\hline DREAM-D150N & $\begin{array}{c}\tau_{1} \\
(\mathrm{~ns})\end{array}$ & $\begin{array}{l}\mathrm{w}_{1} \\
(\mathrm{~ns})\end{array}$ & $\begin{array}{c}f_{1} \\
(\%)\end{array}$ & $\alpha_{1}$ & $\begin{array}{c}\tau_{2} \\
(\mathrm{~ns})\end{array}$ & $\begin{array}{c}f_{2} \\
(\%)\end{array}$ & $\alpha_{2}$ & $\begin{array}{l}\langle\tau\rangle \\
(\mathrm{ns})\end{array}$ & $\chi^{2}$ \\
\hline Apo & 2.9 & $1.29 \pm 0.04$ & 39.2 & $0.62 \pm 0.02$ & 7.4 & 60.8 & $0.38 \pm 0.02$ & 5.6 & 0.9 \\
\hline $\mathbf{M g}^{2+}$ & 2.8 & $1.20 \pm 0.08$ & 34.6 & $0.58 \pm 0.05$ & 7.4 & 65.4 & $0.42 \pm 0.05$ & 5.8 & 0.6 \\
\hline $\mathrm{Ca}^{2+}$ & 1.9 & $0.72 \pm 0.07$ & 16.2 & $0.45 \pm 0.02$ & 7.4 & 83.8 & $0.55 \pm 0.02$ & 6.5 & 0.9 \\
\hline $\mathrm{Ca}^{2+} \mathrm{Mg}^{2+}$ & 1.8 & $0.70 \pm 0.04$ & 16.0 & $0.43 \pm 0.04$ & 7.4 & 84.0 & $0.57 \pm 0.04$ & 6.5 & 1.1 \\
\hline \multicolumn{10}{|l|}{ DREAM-E186Q } \\
\hline Apo & 2.1 & $0.82 \pm 0.08$ & 22.1 & $0.44 \pm 0.01$ & 5.9 & 77.9 & $0.56 \pm 0.01$ & 5.0 & 1.0 \\
\hline $\mathrm{Mg}^{2+}$ & 2.1 & $0.82 \pm 0.04$ & 23.5 & $0.46 \pm 0.01$ & 5.9 & 76.5 & $0.54 \pm 0.01$ & 5.0 & 1.4 \\
\hline $\mathrm{Ca}^{2+}$ & 2.2 & $0.88 \pm 0.03$ & 24.8 & $0.47 \pm 0.01$ & 5.9 & 75.2 & $0.53 \pm 0.01$ & 5.0 & 1.1 \\
\hline $\mathrm{Ca}^{2+} \mathrm{Mg}^{2+}$ & 2.1 & $0.85 \pm 0.07$ & 22.8 & $0.44 \pm 0.05$ & 5.8 & 77.2 & $0.56 \pm 0.05$ & 5.0 & 3.4 \\
\hline \multicolumn{10}{|l|}{ DREAM-E234Q } \\
\hline Apo & 2.5 & $1.11 \pm 0.01$ & 33.1 & $0.54 \pm 0.01$ & 6.3 & 66.9 & $0.46 \pm 0.01$ & 5.0 & 0.7 \\
\hline $\mathrm{Mg}^{2+}$ & 2.4 & $1.00 \pm 0.01$ & 31.3 & $0.55 \pm 0.01$ & 6.3 & 68.7 & $0.45 \pm 0.01$ & 5.0 & 0.8 \\
\hline $\mathrm{Ca}^{2+}$ & 1.8 & $0.76 \pm 0.02$ & 18.7 & $0.45 \pm 0.01$ & 6.3 & 81.3 & $0.55 \pm 0.01$ & 5.4 & 0.9 \\
\hline $\mathrm{Ca}^{2+} \mathrm{Mg}^{2+}$ & 1.8 & $0.74 \pm 0.04$ & 18.8 & $0.45 \pm 0.02$ & 6.3 & 81.2 & $0.55 \pm 0.02$ & 5.4 & 0.9 \\
\hline
\end{tabular}

A constant standard error of $0.2^{\circ}$ for the phase angle and 0.004 for modulation ratio was used. $\tau_{1}$ is the mean decay time of the Gaussian distribution with a width of distribution $w_{1} . \tau_{2}$ is the lifetime of the discrete single exponential term. Values of $\tau_{1}$ and $\tau_{2}$ were determined from the fitting curve of an average intensity decay trace of five measurements. Values of $w, \alpha$, and experimental errors were calculated from the means and standard deviations of an average intensity decay trace of five measurements in at least two independent protein preparations while $\tau_{1}$ and $\tau_{2}$ parameters were linked across.

\subsubsection{Fluorescence intensity decay of 1,8-ANS binding to DREAM mutants}

The fluorescence decay parameters of 1,8-ANS are sensitive to structural changes associated with $\mathrm{Ca}^{2+}$ or $\mathrm{Ca}^{2+} \mathrm{Mg}^{2+}$ binding to DREAM EF-hand mutants. The decay parameters for 1,8-ANS binding to DREAM EF-hand mutants are summarized in Table 7.2. In $20 \mathrm{mM}$ Tris $\mathrm{pH} 7.4$ buffer, 1,8-ANS exhibits a fast lifetime, $\tau_{1}$ of $0.27 \mathrm{~ns}$ and a minor fraction ( 2\%) of 4 ns lifetime due to the non-planar excited state decay (Upadhyay et al. 1995, Someya, Yui 2010). In the presence of DREAM protein, two additional 
lifetimes, $\tau_{2}$ and $\tau_{3}$ were obtained suggesting that 1,8-ANS binds to DREAM at two distinct binding sites with different polarity (Gonzalez and Miksovska, 2014).

The long lifetime, $\tau_{3}$, was associated to 1,8 -ANS bound to a nonpolar solvent restricted binding site on DREAM's surface that promotes the decay of the excited state by populating the long lived non-planar conformation of 1,8-ANS (Gonzalez and Miksovska, 2014). The fast lifetime, $\tau_{2}$, was attributed to the 1,8 -ANS probe bound to a partially solvent exposed binding surface (Gonzalez and Miksovska, 2014).

Intensity decay of 1,8-ANS bound to DREAM EF-hand 2 mutant (DREAMD150N) is shown in Figure 7.8.

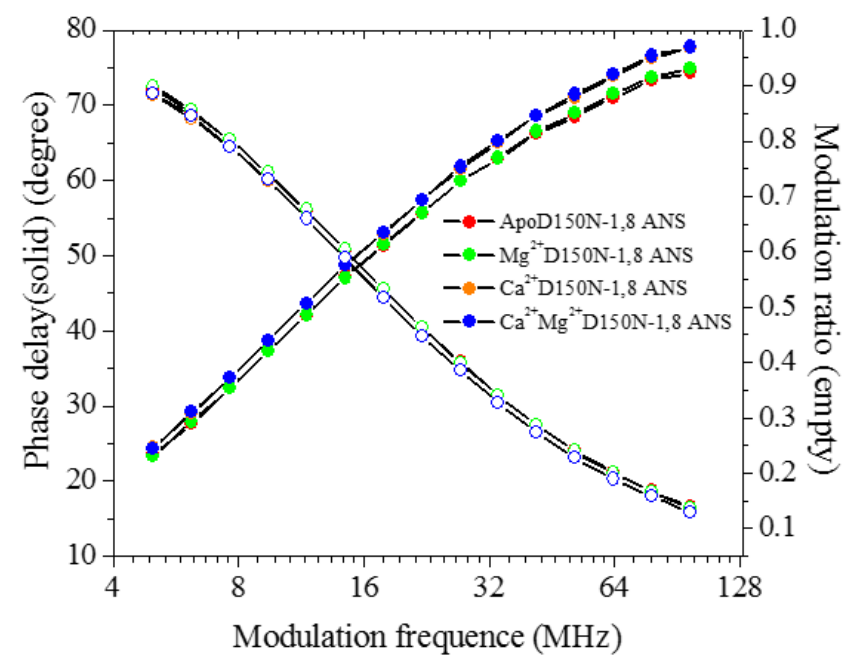

Figure 7.8: Frequency domain intensity decay of 1,8-ANS bound to DREAM-D150N. Phase delay and modulation ratio are shown as solid symbols and empty symbols, respectively. Recovered intensity decay parameters are shown Table 7.2.

Binding of 1,8-ANS to $\mathrm{Ca}^{2+}$ or $\mathrm{Ca}^{2+} \mathrm{Mg}^{2+}$ DREAM-D150N exhibits an increase in its long lifetime $\left(\tau_{3} \sim 18 \mathrm{~ns}\right)$ and pre-exponential value $\left(\alpha_{3} \sim 44 \%\right)$ relative to that of ApoDREAM-D150N ( $\left.\tau_{3} \sim 17 \mathrm{~ns}, \alpha_{3} \sim 27 \%\right)$. The similar values of $\tau_{3}$ and $\alpha_{3}$ were obtained for 1,8-ANS binding to both $\mathrm{Ca}^{2+}$ and $\mathrm{Ca}^{2+} \mathrm{Mg}^{2+}$ DREAM-D150N suggest that 1,8 -ANS 
binding to the identical binding sites in both $\mathrm{Ca}^{2+}$ and $\mathrm{Ca}^{2+} \mathrm{Mg}^{2+}$ forms. The results also suggest that 1,8-ANS association to the DREAM-D150N is independent of deactivated EFhand 2. Interestingly, an increase in the value of $\tau_{3}$ and $\alpha_{3}$ components (18 ns and $37 \%$, respectively) is also obtained for $\mathrm{Mg}^{2+}$ association to ApoDREAM-D150N suggesting that association of $\mathrm{Mg}^{2+}$ at EF-hands 3 and/or 4 in DREAM leads to a change in hydrophobicity similar to that obtained in $\mathrm{Ca}^{2+}$ bound form. These results suggest that change in surface hydrophobicity of DREAM-D150N is dependent on $\mathrm{Ca}^{2+}$ and/or $\mathrm{Mg}^{2+}$ association to EFhands 3 and 4.

Intensity decay of 1,8-ANS bound to DREAM EF-hand 3 mutant (DREAME186Q) and DREAM EF-hand 4 mutant (DREAM-E234Q) are shown in Figure 7.9 (left and right, respectively).
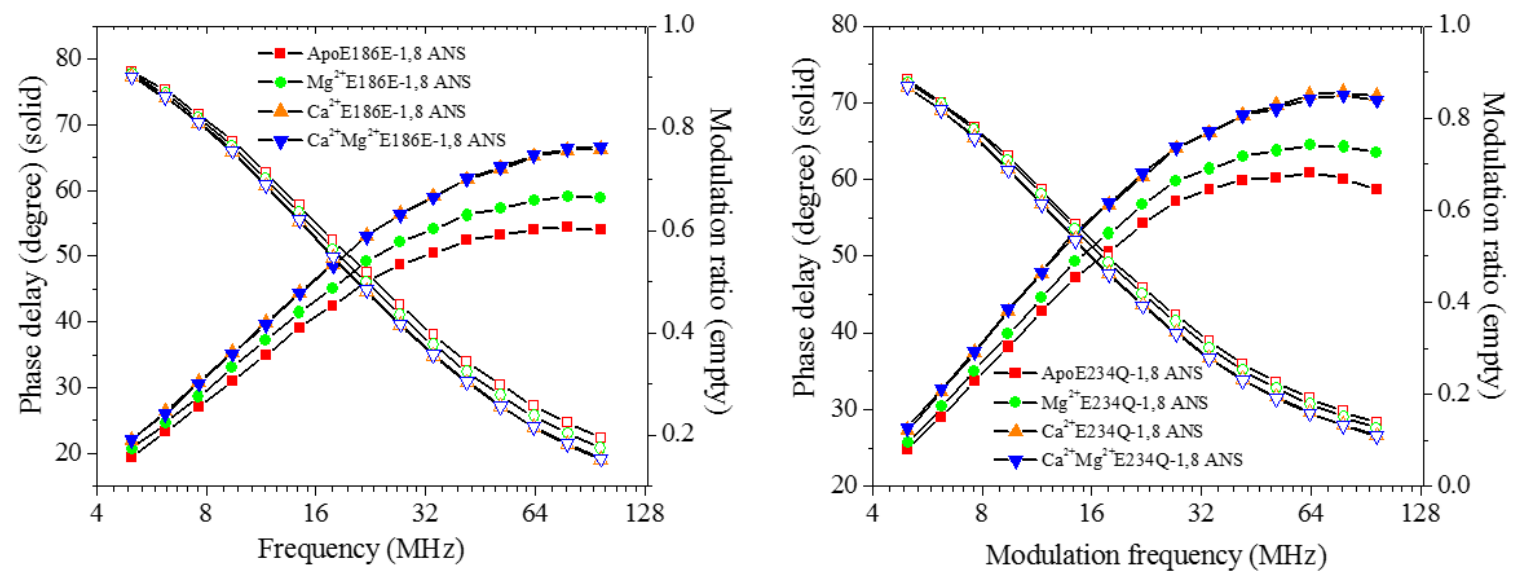

Figure 7.9: Frequency domain intensity decay of 1,8-ANS bound to DREAM-E186Q (left) and DREAM-E234Q (right). Phase delay and modulation ratio are shown as solid symbols and empty symbols, respectively. Recovered intensity decay parameters are shown Table 7.2 .

Binding of 1,8-ANS to DREAM-E186Q and DREAM-E234Q exhibits the same fluorescence intensity decay pattern (Figure 7.9). An increase in the values of $\tau_{3}$ and $\alpha_{3}$ components ( $\sim 16 \mathrm{~ns}$ and $\sim 24 \%$, respectively) is obtained for $\mathrm{Ca}^{2+}$ association to Apo and 
$\mathrm{Mg}^{2+}$ DREAM-E186Q. The results suggest that $\mathrm{Ca}^{2+}$ association solely at EF-hand 3 is able to induce change in DREAM's surface hydrophobicity. Furthermore, $\mathrm{Ca}^{2+}$ association to ApoDREAM-E234Q leads a decrease of $\tau_{2}$ component (4.3 ns) and a small increase in the values $\alpha_{3}$ component (14\%) compared to that of Apo form $\left(\tau_{2}=5.3 \mathrm{~ns}\right.$ and $\left.\alpha_{3}=11 \%\right)$. These results suggest that binding of $\mathrm{Ca}^{2+}$ at EF-hand 4 results in change in DREAM's surface hydrophobicity, however, the surface hydrophobicity change induced by $\mathrm{Ca}^{2+}$ association at EF-hand 4 differs from that of $\mathrm{Ca}^{2+}$ association at EF-hand 3.

Table 7.2: Decay parameters for 1,8-ANS and DREAM EF-hand mutant complexes

\begin{tabular}{|c|c|c|c|c|c|c|c|}
\hline DREAM-D150N & $\begin{array}{l}\tau_{1}{ }^{(*)} \\
(\mathrm{ns})\end{array}$ & $\begin{array}{c}\alpha_{1} \\
(\%)\end{array}$ & $\begin{array}{c}\tau_{2} \\
(\mathrm{~ns})\end{array}$ & $\begin{array}{c}\alpha_{2} \\
(\%)\end{array}$ & $\begin{array}{c}\tau_{3} \\
(\mathrm{~ns})\end{array}$ & $\begin{array}{c}\alpha_{3} \\
(\%)\end{array}$ & $\chi^{2}$ \\
\hline Apo & 0.27 & 48 & 5.2 & 26 & 16.6 & 26 & 1.4 \\
\hline $\mathrm{Mg}^{2+}$ & 0.27 & 28 & 6.6 & 37 & 17.6 & 35 & 1.7 \\
\hline $\mathrm{Ca}^{2+}$ & 0.27 & 25 & 6.6 & 32 & 17.8 & 43 & 1.6 \\
\hline $\mathrm{Ca}^{2+} \mathrm{Mg}^{2+}$ & 0.27 & 24 & 6.6 & 32 & 17.9 & 44 & 1.5 \\
\hline \multicolumn{8}{|l|}{ DREAM-E186Q } \\
\hline Apo & 0.27 & 60 & 5.5 & 22 & 15.2 & 18 & 1.4 \\
\hline $\mathrm{Mg}^{2+}$ & 0.27 & 62 & 5.6 & 21 & 15.7 & 17 & 1.3 \\
\hline $\mathrm{Ca}^{2+}$ & 0.27 & 55 & 5.9 & 21 & 16.4 & 24 & 1.4 \\
\hline $\mathrm{Ca}^{2+} \mathrm{Mg}^{2+}$ & 0.27 & 55 & 6.0 & 22 & 15.9 & 23 & 0.8 \\
\hline \multicolumn{8}{|l|}{ DREAM-E234Q } \\
\hline Apo & 0.27 & 67 & 5.3 & 22 & 14.3 & 11 & 0.3 \\
\hline $\mathrm{Mg}^{2+}$ & 0.27 & 65 & 5.0 & 22 & 13.5 & 13 & 1.2 \\
\hline $\mathrm{Ca}^{2+}$ & 0.27 & 66 & 4.3 & 20 & 13.1 & 14 & 1.9 \\
\hline $\mathrm{Ca}^{2+} \mathrm{Mg}^{2+}$ & 0.27 & 62 & 4.8 & 23 & 13.6 & 15 & 2.7 \\
\hline
\end{tabular}

\subsubsection{Circular dichroism spectra}

Circular dichroism (CD) is an excellent method for characterization of the secondary structural change in DREAM upon $\mathrm{Ca}^{2+}$ and/or $\mathrm{Mg}^{2+}$ association. In this study, the functional role of each EF-hand induces the secondary structure changes that were 
characterized in DREAM EF-hand mutants. Similar to DREAM, the CD spectra of DREAM EF-hand mutants exhibit the strong negative CD signal at the region between 208 $\mathrm{nm}$ and $222 \mathrm{~nm}$ on the spectrum, indicating a high alpha-helical content of the protein. Upon $\mathrm{Ca}^{2+}$ association to ApoDREAM-D150N (Figure 7.10, left panel), a significant decrease in ellipticity between $208 \mathrm{~nm}$ and $222 \mathrm{~nm}$ was observed, which indicates an increase in alpha helical content and/or rearrangement of the alpha helices. On the other hand, $\mathrm{Mg}^{2+}$ association to ApoDREAM-D150N leads to a minor decrease in CD signal suggesting that when EF-hand 2 is inactive, $\mathrm{Mg}^{2+}$ binds to EF-hand 3 and 4, inducing a local rearrangement in alpha helices at its binding site. However, association of $\mathrm{Mg}^{2+}$ to these sites does not significantly alter the secondary structure of DREAM.
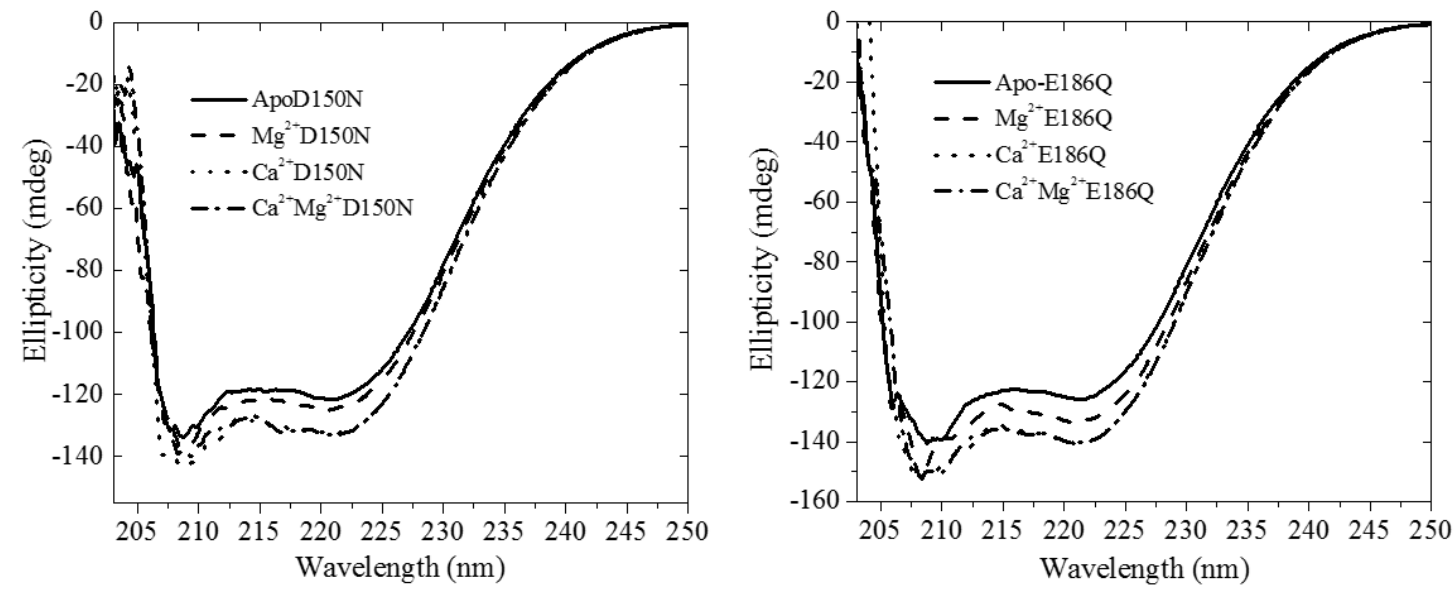

Figure 7.10: Circular dichroism spectra of Apo, $\mathrm{Mg}^{2+}, \mathrm{Ca}^{2+}$, and $\mathrm{Ca}^{2+} \mathrm{Mg}^{2+}$ DREAMD150N (left panel) and DREAM-E186Q (right panel). Conditions: $40 \mu \mathrm{M}$ DREAM in 20 $\mathrm{mM}$ Tris $\mathrm{pH}$ 7.4, $10 \mathrm{mM}$ LDAO, and $1 \mathrm{mM}$ DTT.

On the other hand, $\mathrm{Ca}^{2+}$ association DREAM-E186Q (Figure 7.10, right panel) induces significant secondary structural change similar to that obtained in DREAM and DREAM-D150N. Association of $\mathrm{Mg}^{2+}$ to DREAM-E186Q results in an intermediate CD signal compared to that measured for the Apo and $\mathrm{Ca}^{2+} \mathrm{DREAM}-\mathrm{E} 186 \mathrm{Q}$. Thus, the CD data 
indicate that $\mathrm{Mg}^{2+}$ is able to induce a structural change distinct from those observed for metal free and $\mathrm{Ca}^{2+}$ bound DREAM-E186Q.

Moreover, $\mathrm{Ca}^{2+}$ association to DREAM-E234Q (Figure 7.11) leads to a decrease of ellipticity compared to that observed for the Apo or $\mathrm{Mg}^{2+}$ DREAM-E234Q. Our CD data demonstrate that binding of $\mathrm{Ca}^{2+}$ at either EF-hands 3 or 4 induces significant secondary structural change in DREAM, whereas binding of $\mathrm{Mg}^{2+}$ at EF-hand 2 leads to a local rearrangement in the secondary structure near its binding site.

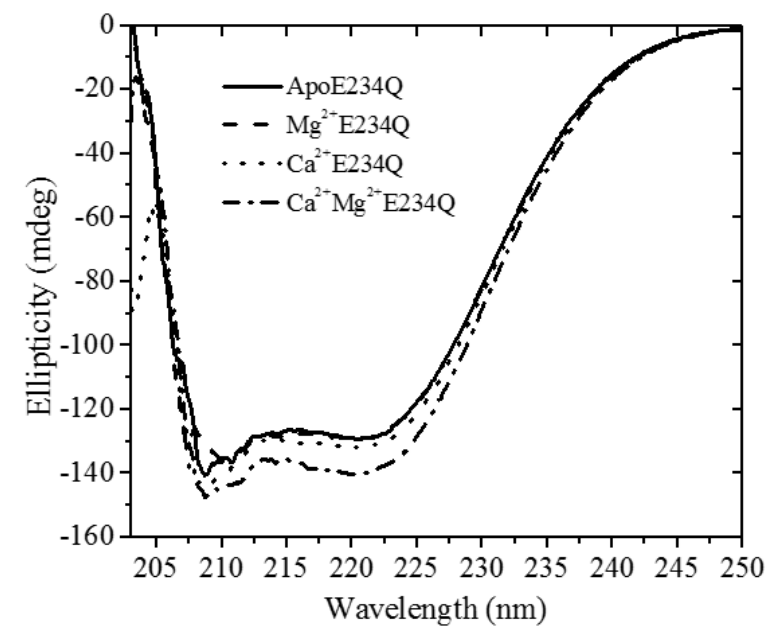

Figure 7.11: Circular dichroism spectra of Apo, $\mathrm{Mg}^{2+}, \mathrm{Ca}^{2+}$, and $\mathrm{Ca}^{2+} \mathrm{Mg}^{2+}$ DREAME234Q. Conditions: $40 \mu \mathrm{M}$ DREAM-E234Q in $20 \mathrm{mM}$ Tris $\mathrm{pH}$ 7.4, $10 \mathrm{mM}$ LDAO, and $1 \mathrm{mM}$ DTT.

\subsubsection{Thermodynamics of DREAM and DNA interaction}

Characterization of the thermodynamics of interactions is important for understanding the biomolecular recognition between DREAM and DNA, as well as the functional role of individual EF-hands in DREAM. The energetics of DREAM and DREAM EF-hand mutants binding to the duplex DNA oligonucleotides corresponding to the DRE sequence of prodynorphin (namely DNA) were carried out using isothermal titration calorimetry (ITC) technique. The isotherms for DNA binding to both ApoDREAM 
and ApoDREAM EF-hand mutants were best fit into a two-sets of sites model, which represents two independent sets of binding affinity between ApoDREAM and DNA. The recovered isotherm parameters are summarized in Table 7.3.

Table 7.3: ITC parameters recovered for DNA binding to DREAM and DREAM EF-hand mutants using a two sets of sites model

\begin{tabular}{|c|c|c|c|c|c|c|}
\hline DREAM and DNA & $\mathrm{n}_{1}$ & $\begin{array}{c}\mathrm{K}_{\mathrm{d} 1} \\
(\mu \mathrm{M})\end{array}$ & $\begin{array}{c}\Delta \mathrm{H}_{1} \\
(\mathrm{kcal} / \mathrm{mol})\end{array}$ & $\mathrm{n}_{2}$ & $\begin{array}{c}\mathrm{K}_{\mathrm{d} 2} \\
(\mu \mathrm{M})\end{array}$ & $\begin{array}{c}\Delta \mathrm{H}_{2} \\
(\mathrm{kcal} / \mathrm{mol})\end{array}$ \\
\hline ApoDREAM & $1.4 \pm 0.1$ & $0.8 \pm 0.1$ & $35 \pm 2$ & $3.3 \pm 0.3$ & $8.5 \pm 0.1$ & $8.8 \pm 2.8$ \\
\hline $\mathrm{Mg}^{2+}$ DREAM & $0.6 \pm 0.1$ & $0.3 \pm 0.1$ & $-18 \pm 1$ & $6.8 \pm 0.8$ & $10 \pm 0.5$ & $0.7 \pm 0.1$ \\
\hline ApoDREAM-D150N & $1.1 \pm 0.1$ & $2.8 \pm 0.8$ & $12 \pm 1$ & $3.4 \pm 1.8$ & $74 \pm 30$ & $15 \pm 11$ \\
\hline ApoDREAM-E186Q & $0.9 \pm 0.1$ & $1.4 \pm 0.5$ & $21 \pm 1$ & $3.5 \pm 1.5$ & $98 \pm 4$ & $39 \pm 28$ \\
\hline ApoDREAM-E234Q & $0.7 \pm 0.1$ & $2.7 \pm 0.2$ & $43 \pm 1$ & $2.8 \pm 0.4$ & $67 \pm 6$ & $50 \pm 9$ \\
\hline$* \mathrm{Ca}^{2+}$ DREAM & 1 & $222 \pm 5$ & $330 \pm 5$ & - & - & - \\
\hline *Ca ${ }^{2+}$ DREAM-D150N & 1 & $264 \pm 6$ & $360 \pm 6$ & - & - & - \\
\hline${ }^{*} \mathrm{Ca}^{2+}$ DREAM-E186Q & 1 & $174 \pm 2$ & $79 \pm 1$ & - & - & - \\
\hline *Ca ${ }^{2+}$ DREAM-E234Q & 1 & $164 \pm 3$ & $86 \pm 1$ & - & - & - \\
\hline
\end{tabular}

ApoDREAM association to DNA (Figure 7.12, panel A) exhibits an endothermic interaction evidenced by positive binding enthalpy $(+\Delta \mathrm{H})$. The first binding site has higher binding affinity $\left(\mathrm{K}_{\mathrm{d} 1}=0.8 \mu \mathrm{M}\right)$ and larger binding enthalpy $\left(\Delta \mathrm{H}_{1}=+35 \mathrm{kcal} / \mathrm{mol}\right)$ compared to that of the second binding site $\left(\mathrm{K}_{\mathrm{d} 1}=8.5 \mu \mathrm{M}, \Delta \mathrm{H}_{1}=+8.8 \mathrm{kcal} / \mathrm{mol}\right)$. In addition, the stoichiometry at the high $\left(\mathrm{n}_{1}\right)$ and low $\left(\mathrm{n}_{2}\right)$ binding affinity sites are $1.4 \pm 0.1$ and $3.3 \pm 0.3$ mole of protein per mole of DNA, respectively. These results suggest an anticooperative mechanism, in which one ApoDREAM molecule binds to DNA with higher affinity that decreases the binding affinity of the other three ApoDREAM molecules for the same DNA. Our isothermal profile for ApoDREAM (residues 65-256) binding to DNA 
(duplex DNA corresponding to the DRE sequence of prodynorphin gene) is similar to that obtained for ApoDREAM mut65-256 (identical amino acid sequence with the ApoDREAM construct in this study) binding to the same DNA (duplex DNA corresponding to the DRE sequence of prodynorphin gene) reported previously (Osawa et al., 2001). The lower binding affinity observed at the first binding site for ApoDREAM binding to DNA $\left(\mathrm{K}_{\mathrm{d}}=0.8 \mu \mathrm{M}\right)$ in our experiment compared to that obtained in the previous study $\left(\mathrm{K}_{\mathrm{d}}=0.2 \mu \mathrm{M}\right)($ Osawa et al., 2001) can be explained by the different temperatures between our experiment $\left(25^{\circ} \mathrm{C}\right)$ and in the previous study $\left(37^{\circ} \mathrm{C}\right)$ (Osawa et al., 2001). The similar tendency for temperature dependence of binding affinity has been observed for binding of polymerase to primed-template DNA (Datta et al., 2006; Datta and LiCata 2003). Nevertheless, the isotherm of ApoDREAM binding to DNA in our study denotes a good reference to investigate the functional roles of individual EF-hand in DREAM in modulating the interaction with DNA.

$\mathrm{Mg}^{2+}$ DREAM exothermically interacts with DNA (Figure 7.12, panel B) with higher binding affinity $\left(\mathrm{K}_{\mathrm{d} 1}=0.3 \mu \mathrm{M}\right)$ and negative binding enthalpy $\left(\Delta \mathrm{H}_{1}=-18\right.$ $\mathrm{kcal} / \mathrm{mole}$ ) at the first binding site compared to that of ApoDREAM. In addition, a high stoichiometry value $\left(\mathrm{n}_{2}=7\right)$, low binding affinity $\left(\mathrm{K}_{\mathrm{d} 2}=10 \mu \mathrm{M}\right)$, and small binding enthalpy $\left(\Delta \mathrm{H}_{2}=0.7 \mathrm{kcal} / \mathrm{mol}\right)$ at the second binding site between $\mathrm{Mg}^{2+}$ DREAM and DNA suggest a non-specific interaction at this binding site. Our ITC results indicate that DNA specifically binds to both Apo and $\mathrm{Mg}^{2+}$ DREAM; however, binding affinity is higher in the $\mathrm{Mg}^{2+}$ form. In addition, different binding thermodynamics of DNA association with Apo and $\mathrm{Mg}^{2+}$ DREAM suggest the different binding mechanisms of interaction between these forms (Holdgate, 2001; Freiburger et al., 2009). 

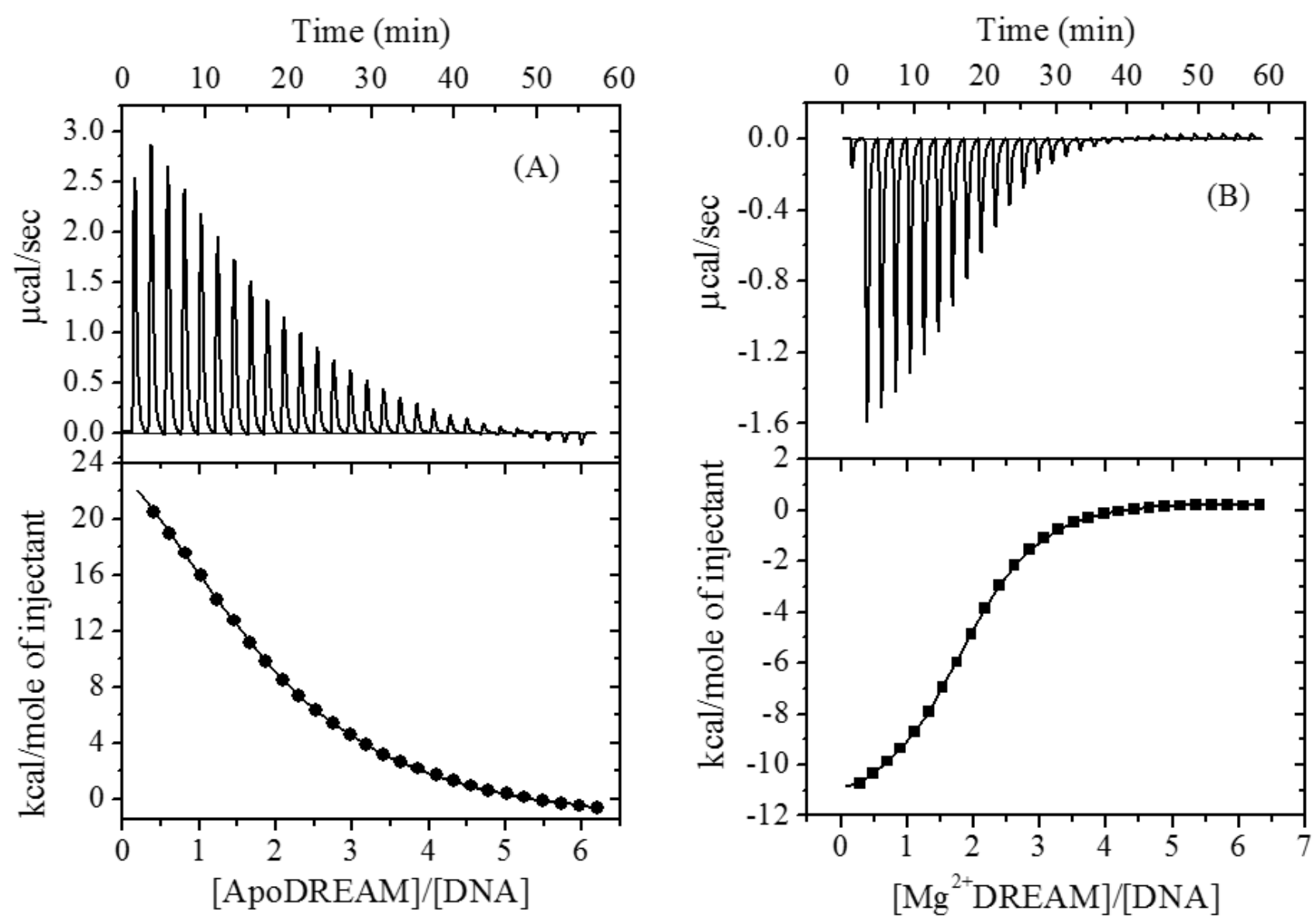

Figure 7.12: ITC isotherms for ApoDREAM (A) and $\mathrm{Mg}^{2+} \mathrm{DREAM}(\mathrm{B})$ binding to DNA. The upper panels of each profile reflects the thermal power expressed in units of $\mu \mathrm{cal} / \mathrm{s}$. The lower panel shows integrated reaction heats $(\Delta \mathrm{H})$ expressed in units of $\mathrm{kcal} / \mathrm{mol}$. The solid lines present the best fitting curve using a two-set of sites model with deletion of the first titration point. The experiment was carried out by titration of $500 \mu \mathrm{M}$ of DREAM protein (in syringe) into $15 \mu \mathrm{M}$ DNA (in sample chamber) at $25^{\circ} \mathrm{C}$. 
To understand the functional properties of each EF-hand of DREAM in modulating the interaction with DNA, ITC experiments were carried out for the association of DNA with DREAM EF-hand mutants. The isotherm profiles for DNA binding to ApoDREAM mutants D150N, E186Q, and E234Q (Figure 7.13 panels: A, B, and C, respectively) are similar to that obtained for ApoDREAM binding to DNA. However, the binding affinity is slightly smaller at the first binding site $\left(\mathrm{K}_{\mathrm{d} 1} \sim 1.4\right.$ to $\left.2.8 \mu \mathrm{M}\right)$ and that is much higher $\left(\mathrm{K}_{\mathrm{d} 2}\right.$ $\sim 67$ to $98 \mu \mathrm{M}$ ) at the second binding site compared to that of ApoDREAM. These results suggest a small structural deviation between ApoDREAM wild-type and mutants (see explanation in discussion). In contrast, the isotherms of DNA association to $\mathrm{Ca}^{2+}$ bound DREAM and DREAM EF-hand mutants can be best modeled using a sequential binding sites model with binding stoichiometry, $\mathrm{n}=1$. No significant binding was observed for DNA association with $\mathrm{Ca}^{2+}$ DREAM wild-type and $\mathrm{Ca}^{2+}$ DREAM-D150N $\left(\mathrm{K}_{\mathrm{d}} \sim 225\right.$ to 265 $\mu \mathrm{M}$ ) (Figure 7.14, panel $\mathrm{A}$ and $\mathrm{B}$, respectively), whereas a higher binding affinity was observed for association of DNA to $\mathrm{Ca}^{2+}$ DREAM-E186Q and $\mathrm{Ca}^{2+}$ DREAM-E234Q $\left(\mathrm{K}_{\mathrm{d}} \sim\right.$ 164 to $174 \mu \mathrm{M}$ ) (Figure 7.14, panels $\mathrm{C}$ and D, respectively). These results suggest that binding of $\mathrm{Ca}^{2+}$ at either EF-hand 3 or 4 in DREAM is able to modulate interaction with DNA, but binding of $\mathrm{Ca}^{2+}$ at both EF-hands 3 and 4 in DREAM fully inhibits the interaction between DREAM and DNA. 

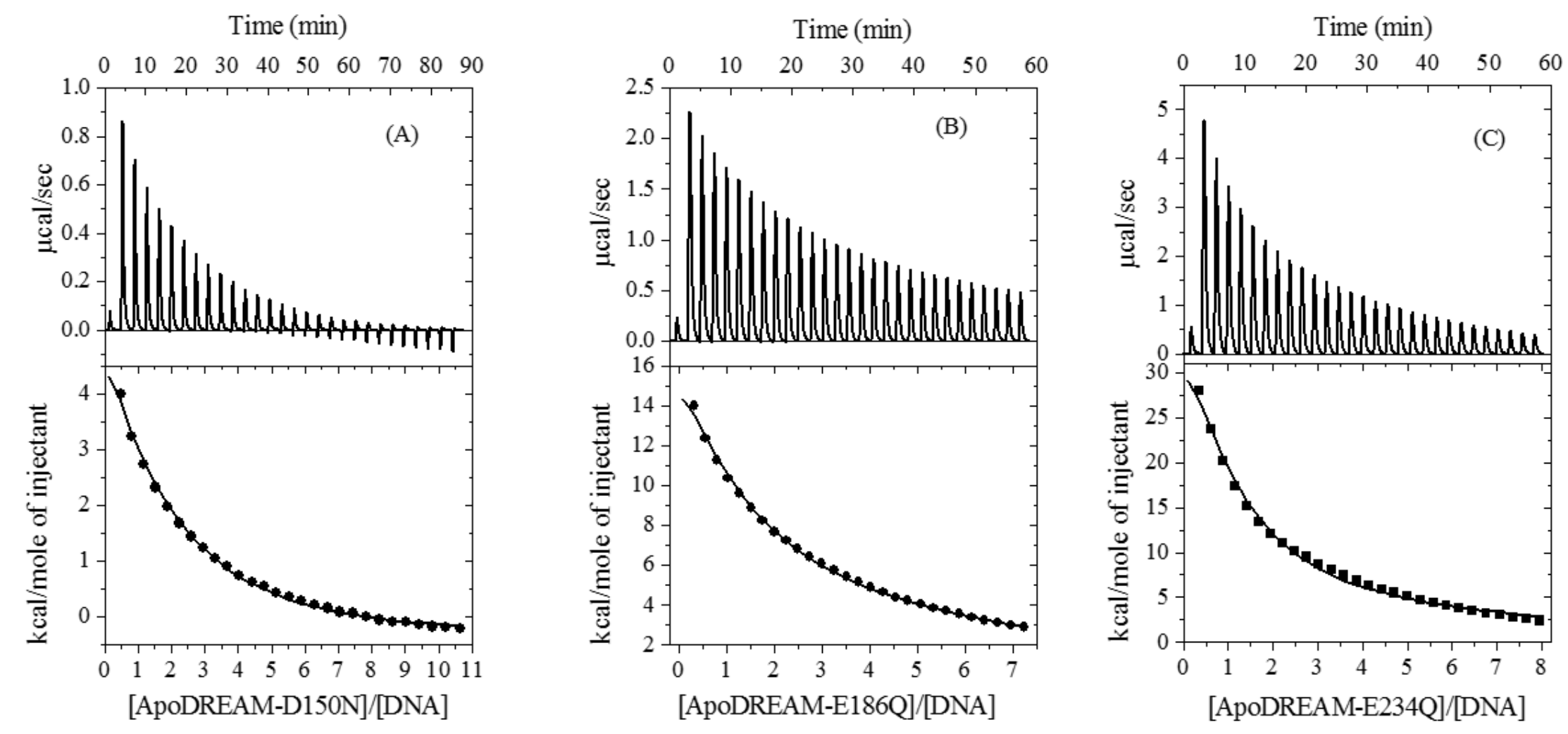

Figure 7.13: ITC isotherms for ApoDREAM-D150N (A) and ApoDREAM-E186Q (B), and ApoDREAM-E234Q (C) binding to DNA. The upper panels of each profile reflects the thermal power expressed in units of $\mu \mathrm{cal} / \mathrm{s}$. The lower panel shows integrated reaction heats $(\Delta \mathrm{H})$ expressed in units of $\mathrm{kcal} / \mathrm{mol}$. The solid lines present the best fitting curve using a two-set of sites model with deletion of the first titration point. The recovered isothermal parameters are shown in Table 7.3. 

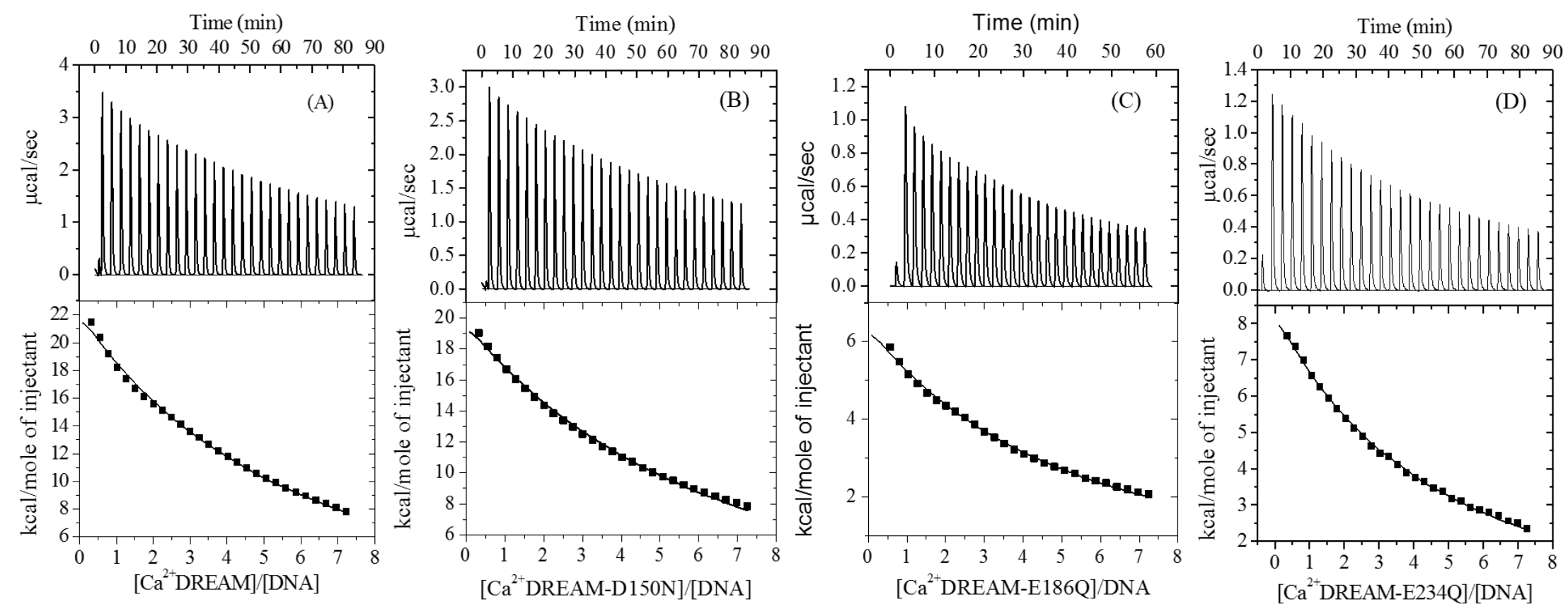

Figure 7.14: ITC isotherms for $\mathrm{Ca}^{2+}$ DREAM (A), $\mathrm{Ca}^{2+} \mathrm{DREAM}-\mathrm{D} 150 \mathrm{~N}$ (B), $\mathrm{Ca}^{2+} \mathrm{DREAM}-\mathrm{E} 186 \mathrm{Q}$ (C), and $\mathrm{Ca}^{2+} \mathrm{DREAM}-\mathrm{E} 234 \mathrm{Q}$ (D) binding to DNA. The upper panels of each profile reflects the thermal power expressed in units of $\mu \mathrm{cal} / \mathrm{s}$. The lower panel shows integrated reaction heats $(\Delta \mathrm{H})$ expressed in units of $\mathrm{kcal} / \mathrm{mol}$. The solid lines present the best fitting curve using a sequential binding sites model with deletion of the first titration point. The recovered isothermal parameters are shown in Table 7.3. 


\subsubsection{Oligomeric states of DREAM in complex with DNA}

Because $\mathrm{Ca}^{2+} / \mathrm{Mg}^{2+}$ occupancy at EF-hands directly changes oligomerization of DREAM, which in turn mediates the interactions with DNA (Osawa et al., 2001; Osawa et al., 2005; Lusin et al., 2008; Gonzalez and Miksovska 2014), the oligomerization states of DNA in complex with ApoDREAM and $\mathrm{Mg}^{2+}$ DREAM were characterized using timeresolved anisotropy. The anisotropy decay traces of DNA in buffer, and DNA in complex with ApoDREAM, $\mathrm{Mg}^{2+}$ DREAM, and $\mathrm{Ca}^{2+}$ DREAM are shown in Figure 7.15 , panel A. The anisotropy decay parameters are shown in Table 7.4. The anisotropy decay of DNA in buffer solution and $\mathrm{Ca}^{2+}$ DREAM-DNA complex were fit into a single correlation time model with rotational correlation time $\left(\theta_{1}\right)$ of $10.7 \mathrm{~ns}$ and $8.1 \mathrm{~ns}$, respectively. Anisotropy decay of ApoDREAM-DNA complex was fit into a two rotational correlation time model, in which the fast rotational correlation time $\left(\theta_{1}\right)$ of $8.1 \mathrm{~ns}$ represents the rotation of DNA free in buffer solution and the slow rotational correlation time $\left(\theta_{2}\right)$ of $54.5 \mathrm{~ns}$ reflects global rotation of ApoDREAM-DNA complex (Table 7.4).

To investigate the interaction of $\mathrm{Mg}^{2+}$ DREAM with DNA, anisotropy decay experiments were carried out in the presence of $100 \mu \mathrm{M}$. The anisotropy decay of DNA in buffer solution containing $\mathrm{Mg}^{2+}$ and the $\mathrm{Mg}^{2+}$ DREAM-DNA complex (Figure 7.15, panel B) complex were fit using a two correlation time model with the fast rotational correlation time $\left(\theta_{1}\right)$ of $2.7 \mathrm{~ns}$ reflecting the local motion of the fluorophore (ethidium bromide) and the slow rotational correlation time $\left(\theta_{2}\right)$ of 21.9 ns representing the global motion of the $\mathrm{Mg}^{2+}$ DREAM-DNA complex (Table 7.4). 

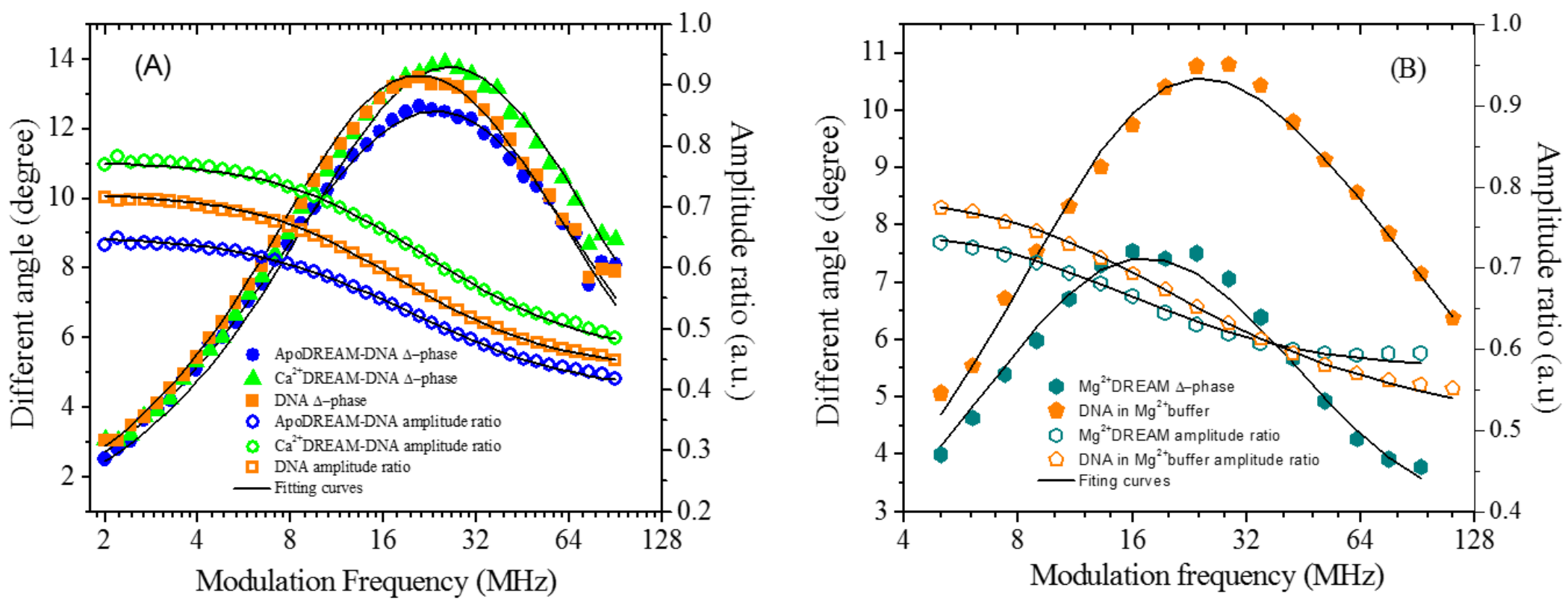

Figure 7.15: A: Time-resolved anisotropy of DNA in buffer solution (triangle), $\mathrm{Ca}^{2+}$ DREAM-DNA complex (square), and ApoDREAMDNA complex (circle). B: Time-resolved anisotropy of DNA in buffer solution containing $100 \mu \mathrm{M} \mathrm{MgCl}_{2}$ (pentagon) and $\mathrm{Mg}^{2+}$ DREAM-DNA complex (hexagon). Differential phase angle and modulation ratio are shown as solid and empty symbols, respectively. Solid lines represent the non-linear least square fit and recovered parameters are shown in Table 7.4. 
Table 7.4: Time-resolved anisotropy decay parameters of DREAM and DNA interaction

\begin{tabular}{|l|c|c|c|c|c|}
\hline & $\theta_{1}(\mathrm{~ns})$ & $\theta_{2}(\mathrm{~ns})$ & $\mathrm{r}_{1}$ & $\mathrm{r}_{2}$ & $\chi^{2}$ \\
\hline DNA in buffer & $10.7 \pm 0.2$ & - & 0.30 & - & 1.1 \\
\hline ApoDREAM-DNA & $8.1 \pm 0.2$ & $54.5 \pm 6.0$ & 0.22 & 0.11 & 0.9 \\
\hline $\mathrm{Ca}^{2+}$ DREAM-DNA & $8.1 \pm 0.1$ & - & 0.28 & - & 2.7 \\
\hline DNA in $\mathrm{Mg}^{2+}$ buffer & $2.8 \pm 0.2$ & $14.2 \pm 0.6$ & 0.12 & 0.16 & 1.0 \\
\hline $\mathrm{Mg}^{2+}$ DREAM-DNA & $2.7 \pm 0.2$ & $21.9 \pm 0.9$ & 0.13 & 0.16 & 1.2 \\
\hline
\end{tabular}

With the known molecular weight of DNA and DREAM, the rotational correlation time is estimated to be $8 \mathrm{~ns}$ for DNA, $18.5 \mathrm{~ns}$ for monomeric DREAM bound to one DNA molecule, and $50 \mathrm{~ns}$ for tetramer DREAM bound to one DNA molecule, using a degree of hydration of $0.98 \mathrm{ml} \mathrm{g}^{-1}$ for a spherical complex at $20{ }^{\circ} \mathrm{C}$ (Weber, 1953; Fischer et al., 2004). The experimentally determined value of $\theta_{1}$ for unbound DNA in the ApoDREAM and $\mathrm{Ca}^{2+}$ DREAM samples matches well with the estimated $\theta_{1}$ value. However, the $\theta_{1}$ value of DNA in buffer is about 2 ns slower, reflecting rotation of a non-spherical shape of DNA. The determined $\theta_{2}$ value for ApoDREAM-DNA complex (54.5 $\left.\pm 6.0 \mathrm{~ns}\right)$ matches the estimated $\theta_{2}$ value for tetramer DREAM bound one DNA molecule (50 ns), within experimental error. In addition, the determined $\theta_{2}$ value for $\mathrm{Mg}^{2+}$ DREAM-DNA (21.9 ns) is about 2 ns slower compared to the calculated value, suggesting a non-spherical shape of the $\mathrm{Mg}^{2+}$ DREAM-DNA complex.

\subsubsection{Molecular dynamics simulation of DREAM and DREAM EF-hand mutants}

To get insight into the functional roles of individual EF-hands in modulating the

conformational dynamics, the three dimensional (3D) structures of the Apo and $\mathrm{Mg}^{2+}$ and 
$\mathrm{Ca}^{2+}$ bound DREAM are required. Since the structure of the $\mathrm{Ca}^{2+}$ free form exhibits high flexibility and heterogeneity in solution, the 3D structure of $\mathrm{Apo}$ or $\mathrm{Mg}^{2+}$ bound DREAM has not been experimentally determined (Lusin et al., 2008). Alternatively, advances of molecular dynamics (MD) simulations provide a comprehensive approach to model the structures of proteins (Hospital et al., 2015).

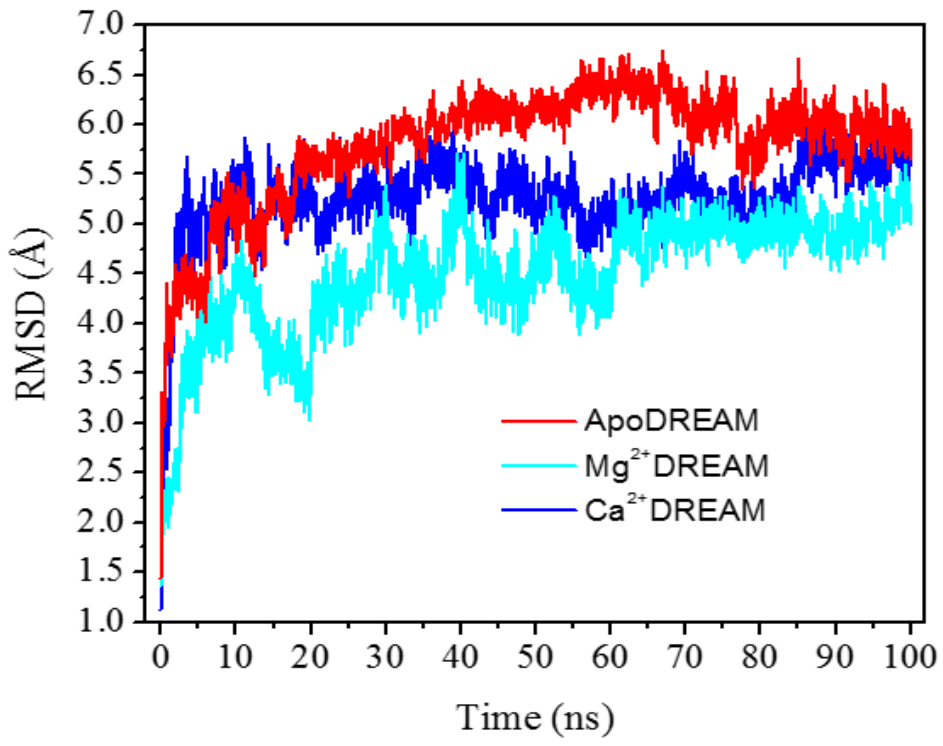

Figure 7.16: The backbone root-mean-square deviations (RMSD) of $\mathrm{Apo}, \mathrm{Mg}^{2+}$, and $\mathrm{Ca}^{2+}$ DREAM during a $100 \mathrm{~ns}$ simulation time.

The MD simulations of Apo and $\mathrm{Mg}^{2+}$ DREAM were constructed using the NMR structure of $\mathrm{Ca}^{2+}$ DREAM as an initial structure. The time evolution of the backbone rootmean-square deviations (RMSD) profile (Figure 7.16) shows that the RMSD of $\mathrm{Ca}^{2+}$ DREAM increased quickly within the first $10 \mathrm{~ns}$, then changed gradually, and reached equilibrium around 4.5 and $5.5 \AA$ after 15 ns simulation time. The backbone RMSD of $\mathrm{Mg}^{2+}$ DREAM shows more flexibility compared to that of $\mathrm{Ca}^{2+}$ DREAM, but changed gradually and remained around 4.5 and $5.0 \AA$ after 60 ns. The RMSD of ApoDREAM exhibits the highest flexibility compared to that of the $\mathrm{Mg}^{2+}$ and $\mathrm{Ca}^{2+}$ bound forms. 
However, the ApoDREAM also reached equilibrium around 5.5 and $6.0 \AA$ after $85 \mathrm{~ns}$ simulation time.

The root-mean-square fluctuation (RMSF) plot of $\mathrm{C}_{\alpha}$ atoms was used to calculate the fluctuations of individual residues with respect to the average structure in Apo, $\mathrm{Mg}^{2+}$, and $\mathrm{Ca}^{2+}$ bound forms of DREAM protein. The RMSF profile (Figure 7.17) shows that binding of $\mathrm{Ca}^{2+}$ at EF- hands 3 and 4 in DREAM reduces the fluctuation of the $\mathrm{Ca}^{2+}$ binding loop of EF hands 3 (residues 170 - 186) and 4 (residue 215 -234), whereas the long flexible loop connecting $\alpha 7$ and $\alpha 8$ (residues 200 - 210) in DREAM remains highly flexible when $\mathrm{Ca}^{2+}$ is bound.

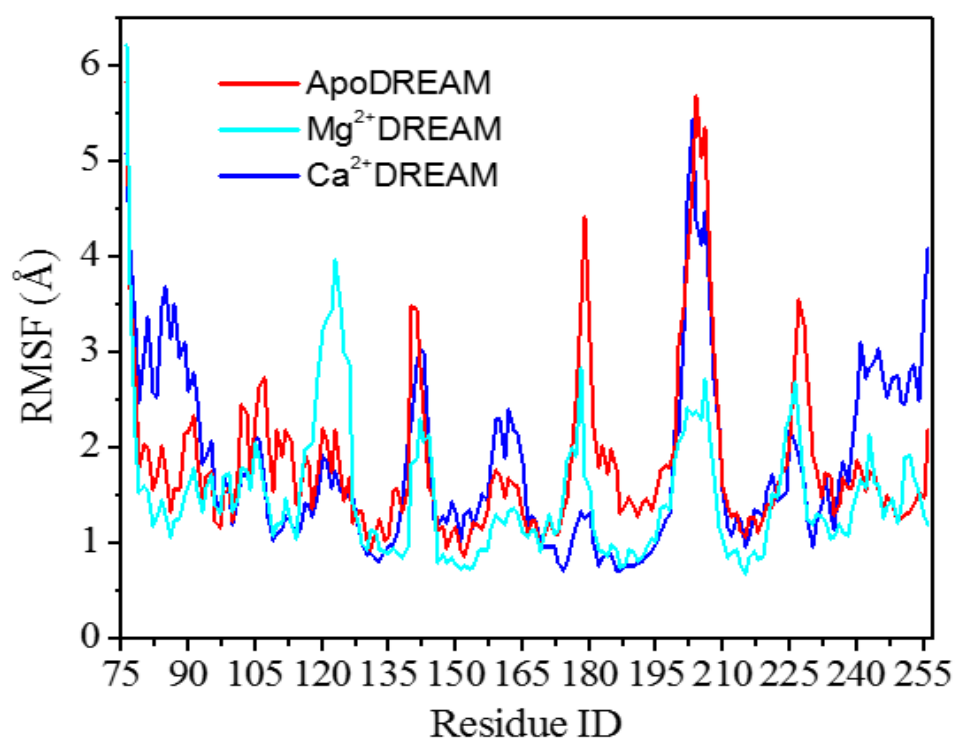

Figure 7.17: The root-mean-square fluctuation (RMSF) plot of $\mathrm{C}_{\alpha}$ atoms represents the fluctuations of individual residues with respect to the average structure in the structures of Apo, $\mathrm{Mg}^{2+}$, and $\mathrm{Ca}^{2+}$ bound forms of DREAM from 20 to $100 \mathrm{~ns}$ simulation time.

These results are in agreement with that observed from the previous MD simulation study in our group (Pham, Dhulipala et al. 2015) and the NMR study reported by Lusin et al. (Lusin et al., 2008), indicating that the force field (CHARMM27) and the MD 
simulation approach in this study are suitable for constructing a 3D model of $\mathrm{Mg}^{2+}$ DREAM. Indeed, the RMSF profile of $\mathrm{Mg}^{2+}$ DREAM shows that when $\mathrm{Mg}^{2+}$ is bound at EF-hand 2, the region containing $\alpha 1, \alpha 2$, and $\alpha 5$ in the N-terminus of DREAM exhibit decrease in fluctuation compare to that of $\mathrm{Apo}$ and $\mathrm{Ca}^{2+}$ bound forms. Interestingly, the loop connecting $\alpha 3$ and $\alpha 4$ (residues 121 - 128) exhibits increased fluctuation, whereas the long loop connecting $\alpha 7$ and $\alpha 8$ (residues 197 -211) and C-terminal regions (residues 216 to 256) are more stable in $\mathrm{Mg}^{2+}$ DREAM.

\subsubsection{Dynamical network analysis of DREAM molecular dynamics simulations}

To better illustrate the dynamic organization of DREAM, I carried out analysis of the MD simulations using dynamic network analysis. This approach allows me to build three dimensional networks which represent the cluster of amino acids whose motions are highly correlated. The resulting networks of Apo, $\mathrm{Ca}^{2+}$ bound at EF-hands 3 and $4, \mathrm{Ca}^{2+}$ bound at EF-hand 3 (DREAM-E234Q mutant), and $\mathrm{Ca}^{2+}$ bound at EF-hand 4 (DREAM186Q mutant) (Figure 7.18).

Dynamical network analysis of ApoDREAM and $\mathrm{Ca}^{2+}$ DREAM simulations (Figure 7.18, top left and right panels, respectively) shows that communities in ApoDREAM remain connected, however, the correlation between communities, represented by thinner edges connecting the nodes, is much weaker compared to that of $\mathrm{Ca}^{2+}$ DREAM. In addition, communities containing $\alpha$-helices $1,2,3,4$, and 5 in the $\mathrm{N}$ terminus (red, yellow, and orange) are rearranged upon calcium binding to either or both EF-hands. The red community, which contains only $\alpha 2$ in the Apo form, is correlated to $\alpha 3, \alpha 5$, and partially with $\alpha 4$ in $\mathrm{Ca}^{2+}$ bound form, creating a compact domain in the $\mathrm{N}$ terminus. Interestingly, in the absence of $\mathrm{Ca}^{2+}$, the $\alpha 10$ helix is coupled to the $\mathrm{N}$-terminal 
domain of DREAM through weak interactions with $\alpha 3$ and $\alpha 4$, whereas it strongly interacts with $\alpha 7$ and $\alpha 8$ in the $\mathrm{Ca}^{2+}$ bound form creating a highly correlated domain in the C-terminus. Analysis of DREAM-E186Q shows that binding of $\mathrm{Ca}^{2+}$ at EF-hand 4 rearranges the purple community, in which $\alpha 8$ becomes connected with $\alpha 6$ and $\alpha 7$. In addition, organization of the red, yellow, and orange communities in the N-terminus remains similar with that of the ApoDREAM.
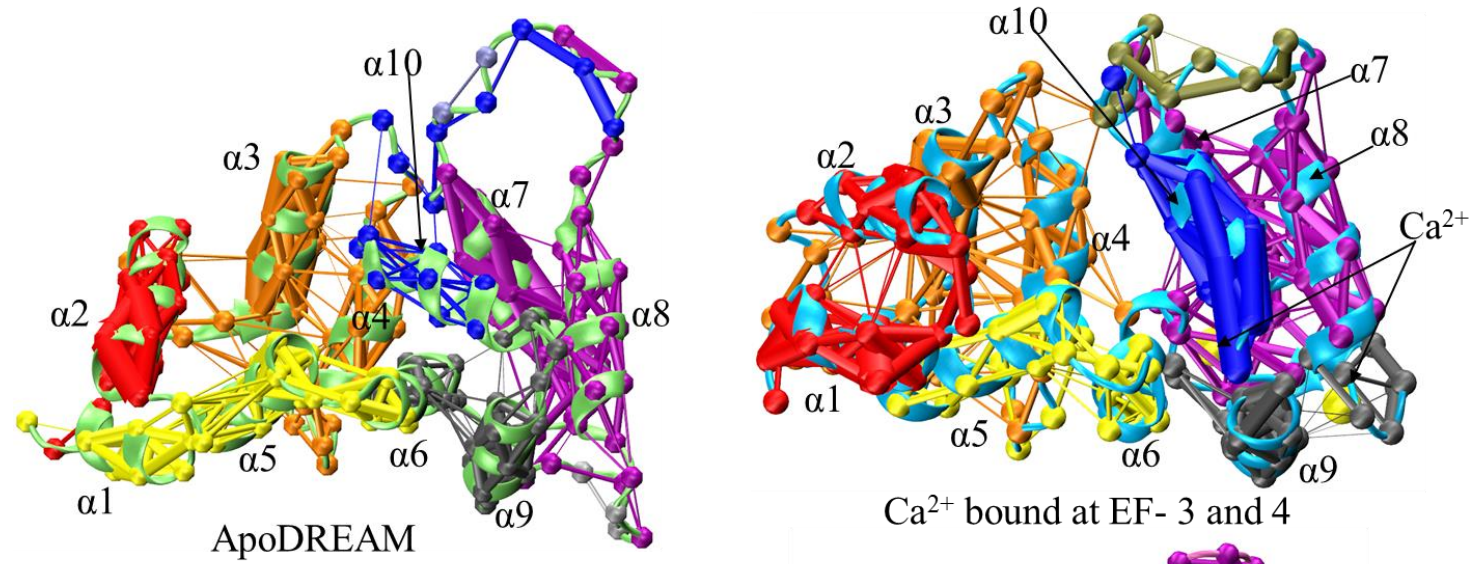

$\mathrm{Ca}^{2+}$ bound at $\mathrm{EF}-3$ and 4

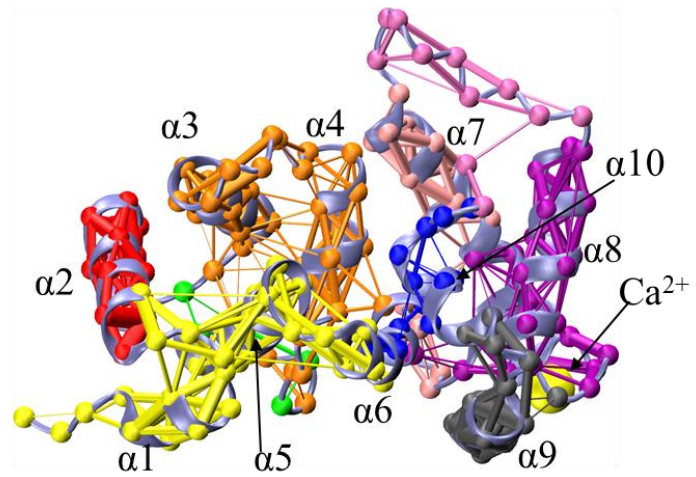

$\mathrm{Ca}^{2+}$ bound at EF-4 (E186Q mutant)

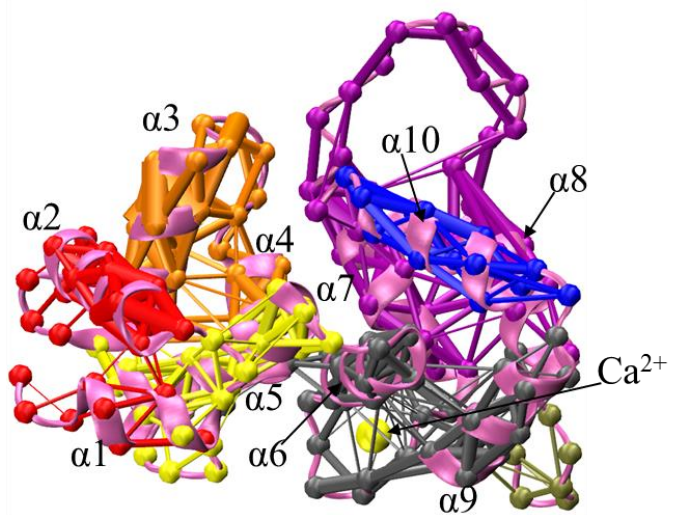

$\mathrm{Ca}^{2+}$ bound at EF-3 (E234Q mutant)

Figure 7.18: Graphic representation of the monomer structures averaged over 100 ns of MD simulations of Apo (green), $\mathrm{Ca}^{2+}$ bound at EF-hands 3 and 4 (blue), $\mathrm{Ca}^{2+}$ bound at EFhand 4 in DREAM-E186Q mutant (light blue), and Ca2+ bound at EF-hand 3 in DREAME234Q mutant (pink). All nodes and edges of the communities are colored and shown as ball and stick, respectively.

On the other hand, analysis of DREAM-E234Q mutant $\left(\mathrm{Ca}^{2+}\right.$ bound EF-hand 3) shows that the gray community, which contains only $\alpha 9$ and/or partial $\alpha 6$ in ApoDREAM, 
$\mathrm{Ca}^{2+}$ DREAM, and $\mathrm{Ca}^{2+}$ DREAM-E186, is significantly spatially reorganized and includes a new connection to a part of $\alpha 5$. Furthermore, the organization of the red, yellow, and orange community in $\mathrm{Ca}^{2+}$ DREAM-E234Q is structurally similar to that of $\mathrm{Ca}^{2+}$ DREAM wild-type, lacking the strong correlation within residues of $\alpha 1$. These results suggest that EF-hand 3 may play an important role in $\mathrm{Ca}^{2+}$ signaling transduction from the $\mathrm{C}$ - to $\mathrm{N}$ terminal domains of DREAM.

\subsubsection{Dynamical paths connecting residues R98 and E186 in DREAM}

Following the results from dynamical network analysis, which suggested that $\mathrm{Ca}^{2+}$ association to EF-hand 3 may play a crucial role in tertiary structure reorganization and propagation of $\mathrm{Ca}^{2+}$ signal transduction from $\mathrm{C}$ - to $\mathrm{N}$-terminal domains in DREAM dynamic pathway was determined between E186, the catalytic residue in EF-hand 3 (Osawa et al., 2005), and R98, located in N-terminal. The residue R98 was chosen for analysis because it was proposed to be critical for DREAM interaction with DNA (Lusin et al., 2008). Therefore, the paths connecting R98 and EF-hand 3 may reflect the paths for $\mathrm{Ca}^{2+}$ signal transduction from EF-hands 3 from the $\mathrm{C}$-terminus to the $\mathrm{N}$-terminus in order to allosterically regulate the interactions with DNA. Experimentally, W169 was employed as an intrinsic fluorophore due to its sensitivity to changes in hydrophobicity of the surrounding environment upon $\mathrm{Ca}^{2+}$ association. Steady-state fluorescence emission and change in fluorescence lifetimes of W169 were monitored and combined with computational results elucidate the functional role of each EF-hand in DREAM. Analysis of ApoDREAM shows a single path connecting residues R98 and E186 (Figure 7.19, top left panel). In addition, the thinner edges connecting the communities in this path represent weak connection. Furthermore, residue W169 is not included in the path connecting R98 
and E186 in ApoDREAM. In contrast, two strongly coupled paths connecting residue R98 and E186 were obtained in $\mathrm{Ca}^{2+}$ DREAM wild-type (Figure 7.19, bottom panel). Further analysis of the paths connecting R98 and E186 in $\mathrm{Ca}^{2+}$ DREAM reveals that they contain the major hydrophobic residues F88, L155, I157, L158, L159, W169, L173, F121, L155, Y118, F114, L134, F135, F133, Y130, and A137 (Figure 1.19, bottom panel), which are strongly coupled and create a large hydrophobic cluster in the center of DREAM, connecting the $\mathrm{N}$ - and $\mathrm{C}$-terminal domains and surrounding the $\mathrm{W} 169$ residue.

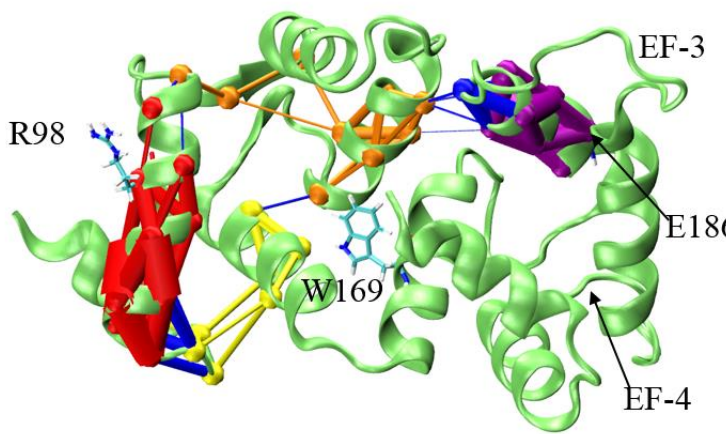

Path from E186 to R98 in ApoDREAM
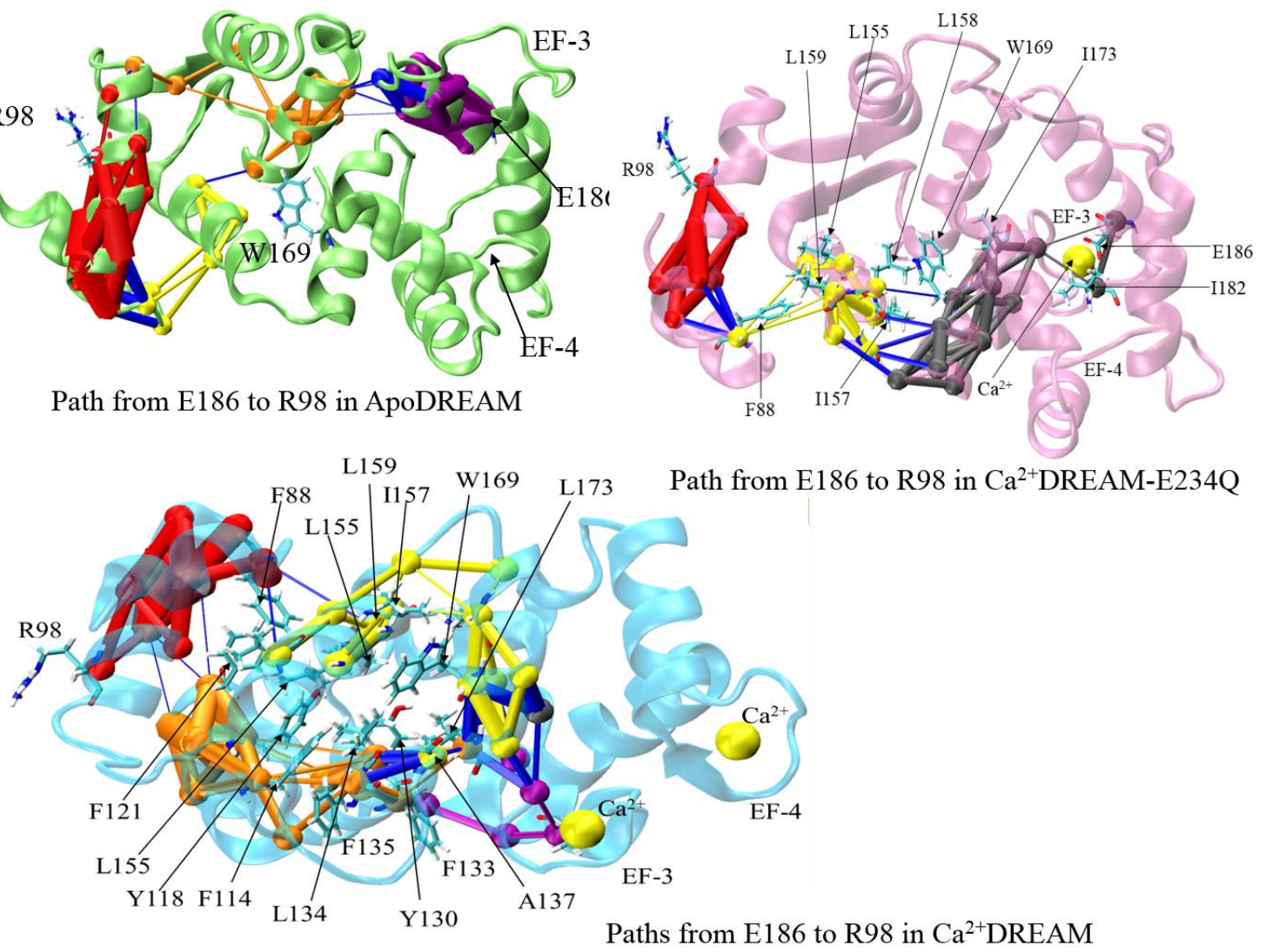

Figure 7.19: Top: The path connecting residues E186 and R98 in ApoDREAM (left), $\mathrm{Ca}^{2+}$ DREAM-E234Q mutant (right), and $\mathrm{Ca}^{2+}$ DREAM wild-type (bottom). The nodes in communities are shown as balls. Communities are colored using the same color codes as shown in Figure 7.18. The hydrophobic residues in the path connecting from R98 to E186 in $\mathrm{Ca}^{2+}$ DREAM wild-type are shown in stick (bottom). 
Furthermore, analysis of DREAM-E234Q $\left(\mathrm{Ca}^{2+}\right.$ bound at EF-hand 3) reveals a single path connecting residues R98 and E186 (Figure 7.19, top right panel). Interestingly, the path contains hydrophobic residues F88, L155, I157, L158, L159, W169, and L173 which are also observed in $\mathrm{Ca}^{2+}$ DREAM wild-type.

Computational results show structural reorganization in DREAM when $\mathrm{Ca}^{2+}$ is bound at EF-hands 3 and 4. This conformational change is accompanied by rearrangement of $\alpha$-helices 4, 5, 7, 8, and 10, which allow residues L155, I157, L158, L159, L134, Y130, Y118, and L173 to form a hydrophobic pocket around W169 in the $\mathrm{Ca}^{2+}$ bound form. Indeed, the fluorescence steady-state emission and lifetime data show a decrease in emission intensity as well as a $5 \mathrm{~nm}$ hypsochromic shift, along with decrease in fluorescence lifetime of W169 which indicates that the residue W169 is buried in a less polar environment compared to their Apo forms (section 7.2.1). These results highlight the role of EF-hand 3 in $\mathrm{Ca}^{2+}$ signal transduction in DREAM.

\subsubsection{Dynamical paths connecting residues R98 and E234 in DREAM}

In parallel, dynamical network analysis was performed to determine contribution of EF-hand 4 in $\mathrm{Ca}^{2+}$ signaling. The computational results show a single path connecting residues R98 and E234Q in ApoDREAM, $\mathrm{Ca}^{2+}$ DREAM wild-type, $\mathrm{Ca}^{2+}$ DREAM-E186Q mutant (Figure 7.20).

The path between R98 and E234 in ApoDREAM is weakly coupled, which is represented by the thin edges connecting the nodes between communities. On the other hand, a strongly coupled path between R98 and E234 was observed in $\mathrm{Ca}^{2+}$ DREAM wildtype and is comprised of hydrophobic residues F88, V152, L159, I157, W169, F235, L236, I232, L167, and F171 (Figure 7.20, bottom panel). 


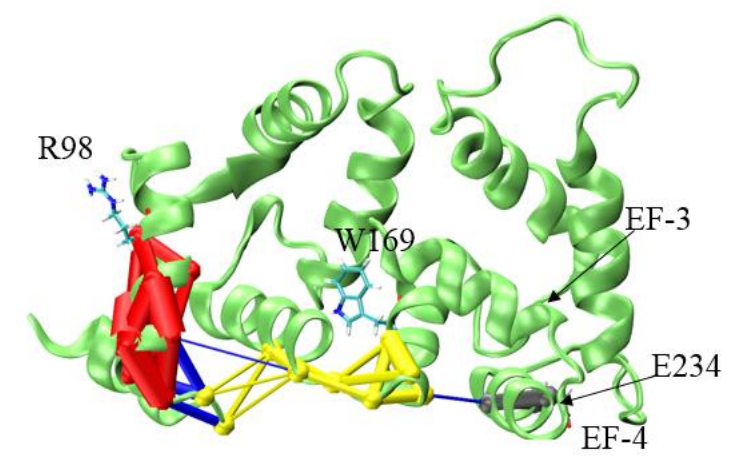

Path from R98 to E234 in ApoDREAM

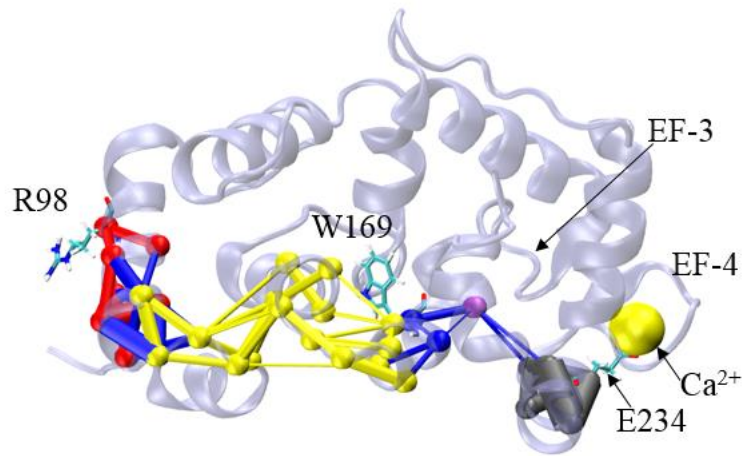

Path from R98 to E234 in $\mathrm{Ca}^{2+}$ DREAM-E186Q

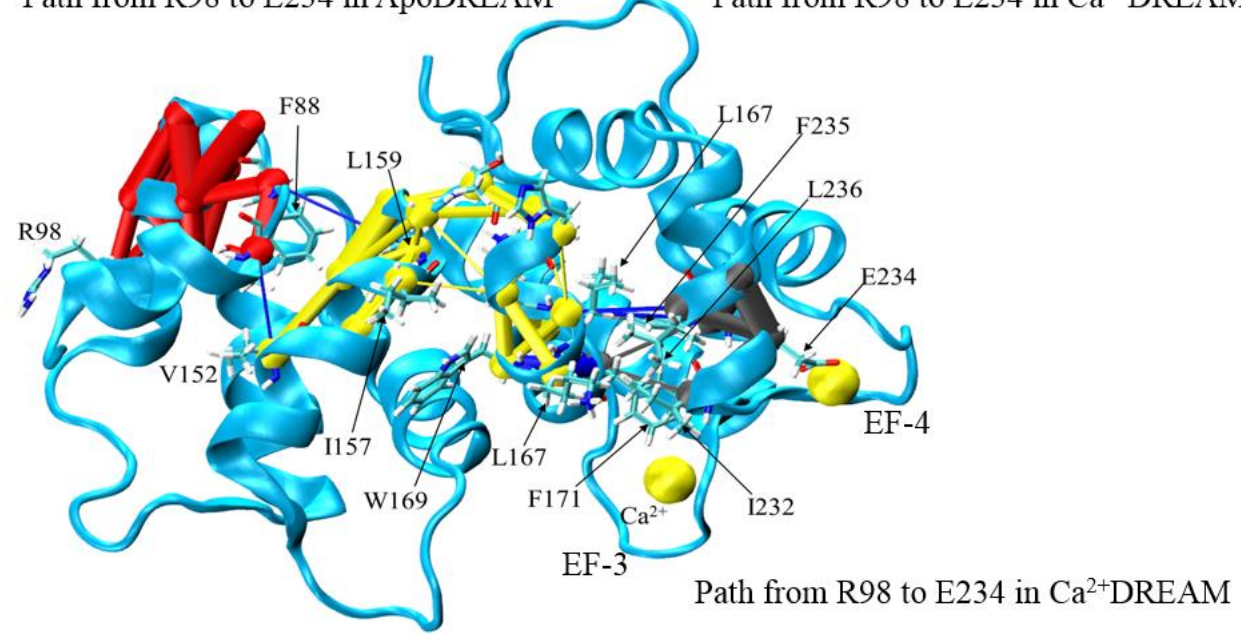

Figure 7.20: The path connecting residues R98 and E234 in ApoDREAM (green), $\mathrm{Ca}^{2+}$ DREAM (blue). The nodes in communities are shown as balls. Communities are colored using the same color scheme as shown in Figure 7.18. The hydrophobic residues within paths connecting from R98 to E234 in $\mathrm{Ca}^{2+}$ DREAM are shown in stick. Note that only two hydrophobic residues I157 and L167 surrounding W169 were observed in the path.

Interestingly, only two hydrophobic residues I157 and L167 surrounding the residue W169 were observed in this path, suggesting that binding of $\mathrm{Ca}^{2+}$ at EF-hand 4 may not significantly alter the hydrophobic environment surrounding W169. Moreover, the path connecting residues R98 and E234 in $\mathrm{Ca}^{2+}$ DREAM-E186Q mutant shares some common residues with that of $\mathrm{Ca}^{2+}$ DREAM wild-type. However, a strongly coupled hydrophobic cluster formed by residues L167, F171, I232, F235, and L236 was obtained in $\mathrm{Ca}^{2+}$ DREAM wild-type (Figure 7.20, bottom panel). 


\subsubsection{2 $\mathrm{Mg}^{2+}$ association to EF-hand 2 stabilizes the $\mathrm{N}$-terminal domain}

Previous studies have shown that binding of $\mathrm{Mg}^{2+}$ at EF-hand 2 in DREAM stabilizes the monomeric form of the protein, whereas ApoDREAM mainly forms a tetramer (Osawa et al., 2005; Gonzalez and Miksovska, 2014). However, the absence of $\mathrm{Mg}^{2+}$ DREAM structure prevents us from understanding how $\mathrm{Mg}^{2+}$ binding to EF-hand stabilizes the DREAM monomer, as well as the influence of $\mathrm{Mg}^{2+}$ on the interaction with DNA. In this study, MD simulation and dynamical network analysis were employed to provide insight into structural changes initiated upon $\mathrm{Mg}^{2+}$ association. Then, the functional role of EF-hand 2 in the modulating DREAM-DNA interaction was elucidated.

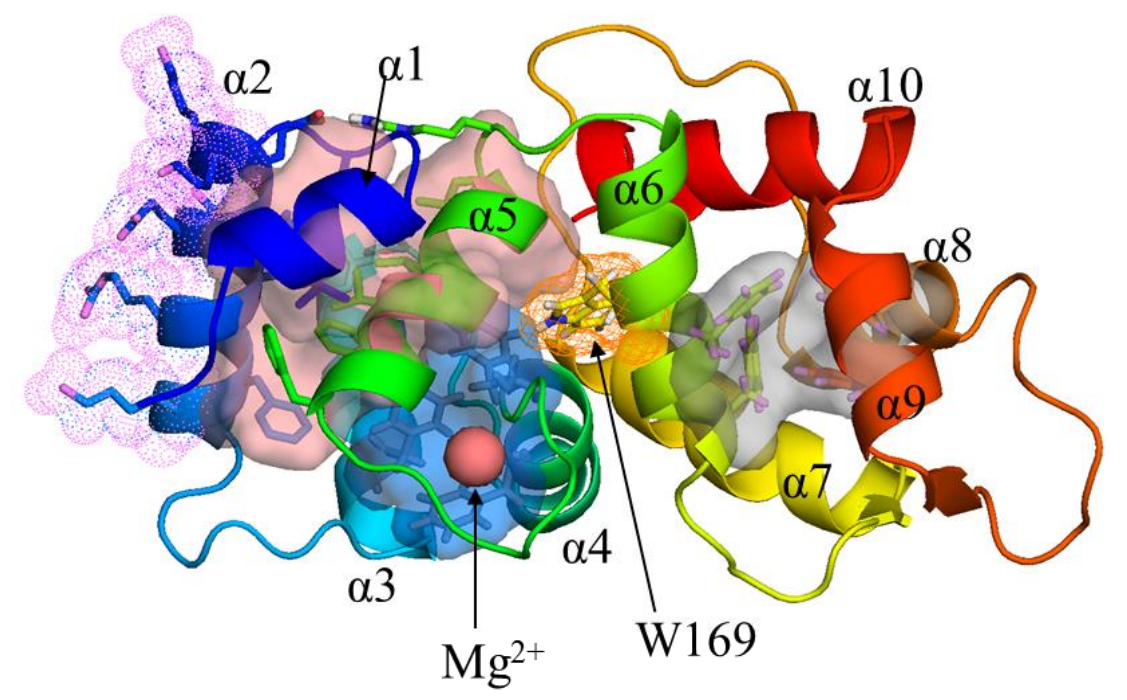

Figure 7.21: The MD simulation model of $\mathrm{Mg}^{2+}$ DREAM: Association of $\mathrm{Mg}^{2+}$ at EF-hand 2 forms a new hydrophobic cluster (shown as pink solid surface), that has not been observed in either Apo or $\mathrm{Ca}^{2+}$ DREAM. The other two aromatic clusters identified in the $\mathrm{C}$-terminus and $\mathrm{N}$-terminus are conserved in the NMR structure of $\mathrm{Ca}^{2+}$ DREAM and shown as grey solid surface and blue solid surface.

The MD simulation of $\mathrm{Mg}^{2+}$ DREAM reveals that binding of $\mathrm{Mg}^{2+}$ at EF-hand 2 results in the formation of a new hydrophobic cluster formed by hydrophobic residues L93, 
L96, F100, F148, F151, V151, L155, and L159 (shown as pink surface cluster in Figure 7.21 which has not been observed in the NMR structure of $\mathrm{Ca}^{2+}$ DREAM (Lusin et al., 2008).

The presence of a hydrophobic cluster is consistent with the dynamical network analysis that reveals a large network reorganization at the $\mathrm{N}$-terminus upon $\mathrm{Mg}^{2+}$ binding. Specifically, $\alpha 1, \alpha 2$ and $\alpha 5$ (the exiting $\alpha$-helix of EF-hand 2) form a strongly coupled community that is shown in Figure 7.22, left panel.

It is important to note that binding of $\mathrm{Mg}^{2+}$ at $\mathrm{EF}$-hand 2 changes the orientation of DNA-interacting helix $\alpha 2$ so that the hydrophobic residues L93, L96, and F100 on the inner side of $\alpha 2$ are in contact with the hydrophobic residues F121 (from $\alpha 3$ ), F148, F151, V151, L155, and L159 from $\alpha 5$ (exiting helix of EF-hand 2), creating a strongly coupled network. Binding of $\mathrm{Mg}^{2+}$ triggers reorganization of the $\mathrm{N}$-terminus domain which results in an increased exposure of basic and polar residues K90, K91, Q94, S95, R98, K101 on the solvent exposed side of $\alpha 2$ (Figure 7.22, right panel). A salt bridge between residues E92 from $\alpha 2$ and R160 from $\alpha 5$ is observed in the structure of Apo (Figure 7.23, left panel) and $\mathrm{Mg}^{2+}$ DREAM (Figure 7.22, left panel), absent in the average MD structure of $\mathrm{Ca}^{2+}$ DREAM, may contribute to the stabilization of $\alpha 2$ and could lead to the increased stability of ApoDREAM and $\mathrm{Mg}^{2+}$ DREAM-DNA complex (see ITC experimental results).

The orientation of $\alpha 2$ in ApoDREAM and $\mathrm{Mg}^{2+}$ DREAM is somewhat similar to that of the basic helix-loop-helix DNA binding motif, in which exposed residues on the basic helix form sequence-specific contacts with the DNA backbone (Ma et al., 1994; Lusin et al., 2008). Interestingly, residues L155 and L159 from $\alpha 5$, which are proposed to interact with $\mathrm{L} 251$ on the second $\mathrm{Ca}^{2+}$ DREAM molecule to form a $\mathrm{Ca}^{2+}$ DREAM dimer (Lusin et 
al., 2008), are inserted into the N-terminal hydrophobic cluster surrounded by F87, F100, F121, F148, and F151 in $\mathrm{Mg}^{2+}$ DREAM. The structure of the ApoDREAM tetramer is unknown, however it is possible that L155, L159 and L251 are involved in the stabilization of ApoDREAM tetramer suggesting that $\mathrm{Mg}^{2+}$ association at EF-hand 2 precludes the protein from forming a tetramer in the absence of $\mathrm{Ca}^{2+}$. These observations are in accordance with the experimental results, showing that $\mathrm{Mg}^{2+}$ stabilizes DREAM as a monomer, previously reported by our group (Gonzalez and Miksovska, 2014) and Osawa et al. (Osawa et al., 2005).
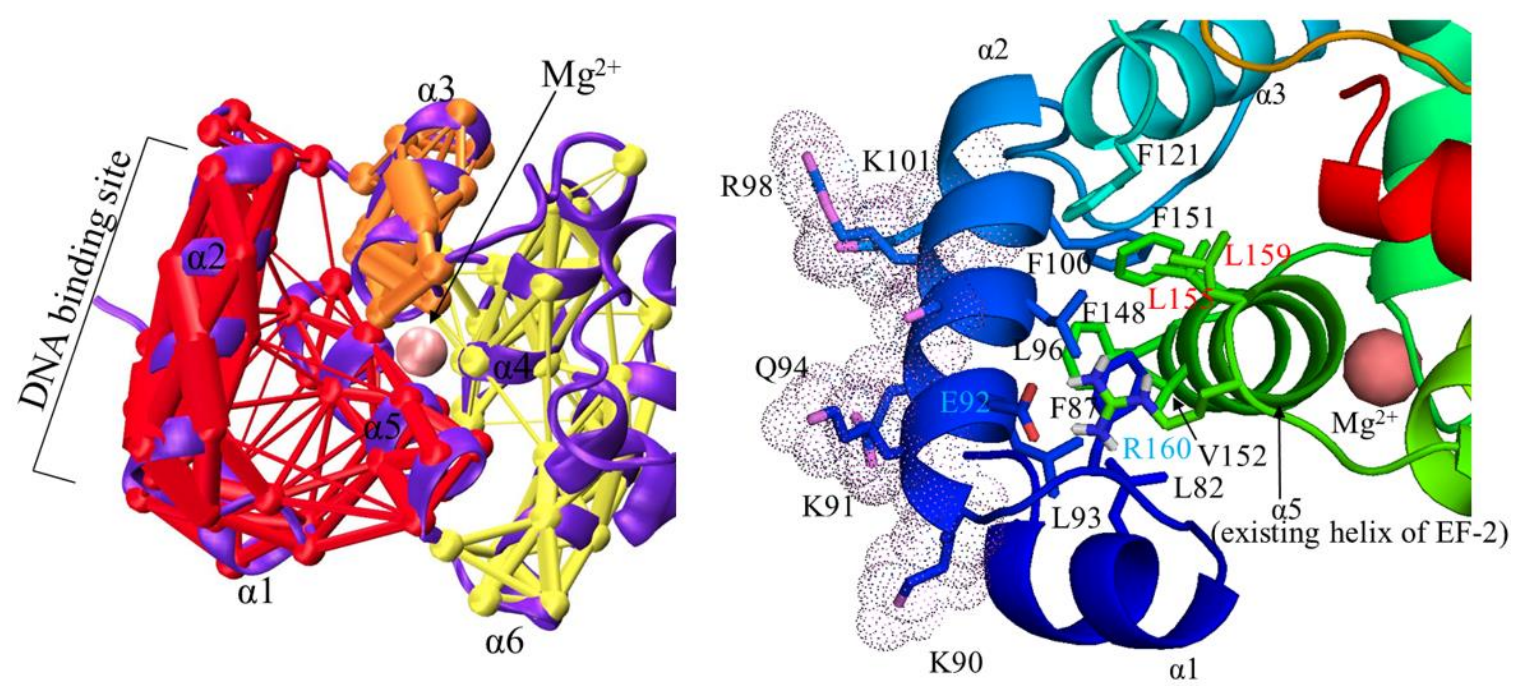

Figure 7.22: Left: Dynamical network analysis of the 100ns MD simulation of the first 6 $\alpha$-helices in $\mathrm{Mg}^{2+} \mathrm{DREAM} \mathrm{N}$-terminus displayed in communities (each community has different color). Right: The dynamical network Hydrophobic cluster L93, L96, and F100 on the inner side of $\alpha 2$ contact with the hydrophobic residues F121 (from $\alpha 3$ ), F148, F151, V151, L155, and L159 from $\alpha 5$ (exiting helix of EF-hand 2) that is stabilized upon $\mathrm{Mg}^{2+}$ association to DREAM. The basic and polar residues K90, K91, Q94, S95, R98, and K101 interfacing with DNA are shown on the outer site of $\alpha 2$. The leucine residues L155 and L159 at the dimeric interface of DREAM are in red, whereas the basic residues forming a salt bridge between $\alpha 2$ and $\alpha 5$ are shown in light blue. 
On the other hand, dynamical network analysis of the MD simulation structure of ApoDREAM reveals that $\alpha 2$ is not involved in an extended community, suggesting a decrease in stability of the N-terminus domain (Figure 7.23, right panel).
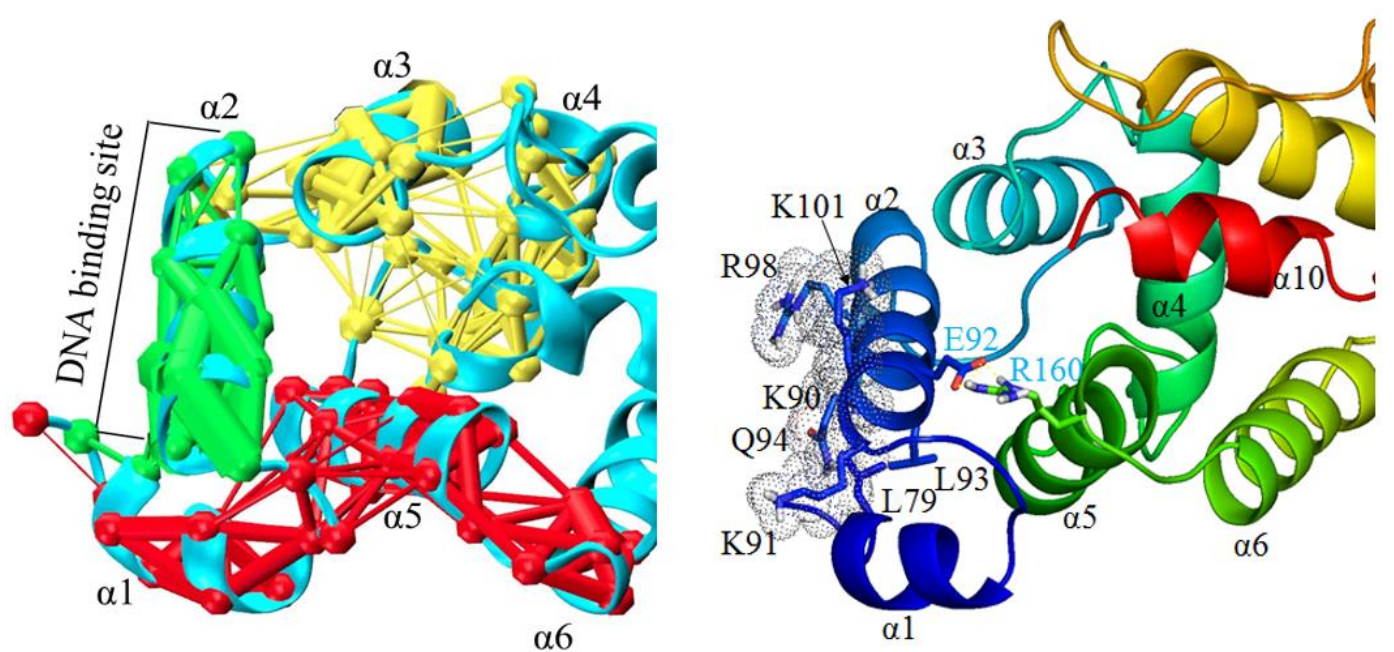

Figure 7.23: Left: Dynamical network analysis of the 100ns MD simulation of the first 6 $\alpha$-helices in ApoDREAM N-terminus displayed in communities (each community colored differently). Right: In the absence of $\mathrm{Mg}^{2+}$, the $\alpha 2$ is not involved in an extended community formed by $\alpha 1, \alpha 5$, and $\alpha 6$, suggesting a decrease in stability of the $\mathrm{N}$-terminus domain despite of the salt bridge formation between residues E92 and R160 (in light blue, bottom).

In contrast to $\mathrm{Mg}^{2+} \mathrm{DREAM}$, dynamical network analysis of the MD simulation structure of $\mathrm{Ca}^{2+}$ DREAM reveals that binding of $\mathrm{Ca}^{2+}$ at EF-hand 3 and 4 leads to the breakage of salt bridge between residue E92 and R160 as well as the absence of the of the extended community formed by $\alpha 1, \alpha 2$, and $\alpha 5$. Indeed, in the structure of $\mathrm{Ca}^{2+}$ DREAM, $\alpha 1$ is repositioned in such way that partially blocks the DNA binding site on $\alpha 2$, resulting in the decrease of DREAM affinity for DNA (Figure 7.24). 


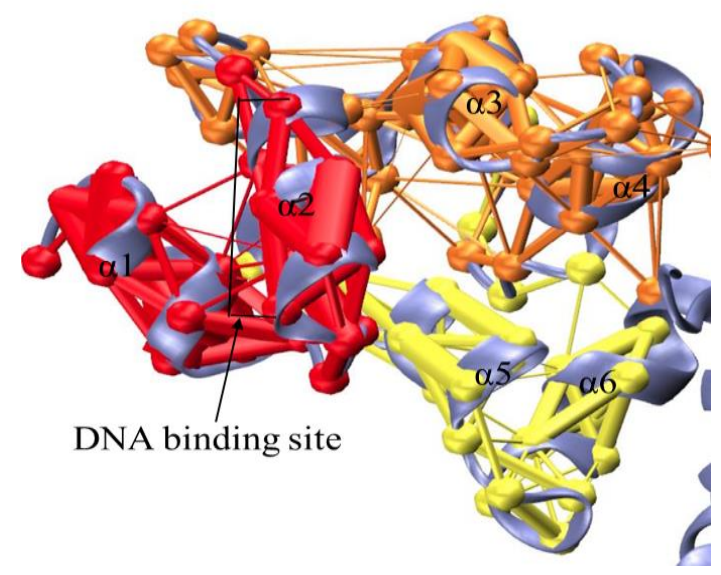

Figure 7.24: Dynamical network analysis of the 100ns MD simulation of the first $6 \alpha$ helices in $\mathrm{Ca}^{2+}$ DREAM N-terminus displayed in communities (each community colored differently). Rearrangement of $\alpha 1$ partially blocks the DNA binding site on $\alpha 2$.

\subsubsection{Computational docking of ApoDREAM and DNA}

The computational docking complex of ApoDREAM and DNA is shown in Figure 7.25. The docking complex of ApoDREAM-DNA reveals that the assembly of ApoDREAM and DNA involves two major types of interaction. Electrostatic interactions are found between the negatively charged phosphate oxygens from nucleotides C43, A13, and C41 from DNA and the positively charged side chain of residues K90, K91, and K101 from $\alpha 2$ in DREAM, respectively.

The complex is further stabilized by hydrogen bonds between residue R98 and nucleotides T11, C12, and C41 with the total energy of $14.3 \mathrm{kcal} / \mathrm{mol}$, determined using YASARA Structure program version 13.1.25 (Krieger et al., 2002), in which the planar guanidinium group of residue R98 from DREAM inserts into the minor groove at the target DRE site formed by 5'-10 GTCA ${ }^{13}-3$ ' and ' $3-{ }^{41} \mathrm{CAGT}^{38}-5$ ' (Carrion, 1999; Ledo et al., 2000) (Figure 7.23). Moreover, the cation- $\pi$ interactions was also obtained between residue R98 from DREAM and nucleotides T11, C12, A40, and C41 from DNA with the total 
interaction strength of $10.6 \mathrm{kcal} / \mathrm{mol}$. Hydrogen bond interactions were also found between pairs of amino acid residue-nucleotide: K90-C43 (5.8 kcal/mol), K91-A13 (5.2 kcal/mol), K101-A41 (5.1 kcal/mol), Q94-T42 (3.4 kcal/mol), S95-A13 (5.7 kcal/mol), and Q120G15 (4.5 kcal $/ \mathrm{mol})$ (Figure 7.23). Furthermore, the residues and nucleotides at interface hot spots determined in the ApoDREAM-DNA docking complex are consistent with the basic residues proposed in other studies (Carrion et al., 1999; Lusin et al., 2008).

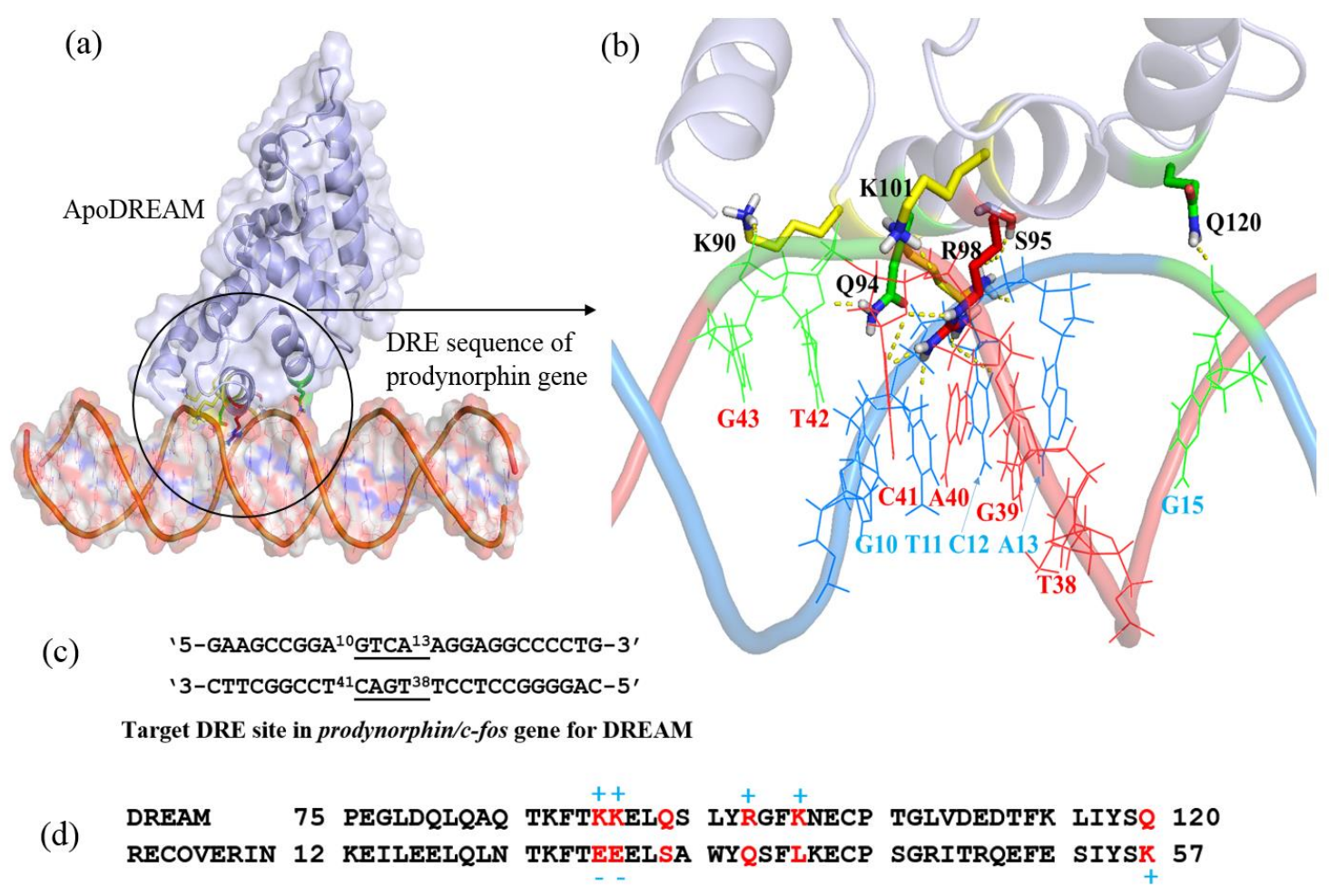

Figure 7.25: (a): The MD simulation of docking complex between ApoDREAM (residues 75-256) and 25 oligomers corresponding to the DRE sequence of prodynorphin gene (namely DNA). (b): A closer view at the interaction interface between ApoDREAM and DNA. Yellow dot lines represent hydrogen bond interactions. (c): the sequence of 25 oligonucleotides corresponding to the DRE. (d): the sequence alignment of 45 amino acid residues in the N-terminus of DREAM (residues: 75-120, top) and that of the non-DNA binding recoverin (residues: 12-57) to highlight the positively charged residues in DREAM participating to the interaction with DNA. 


\subsection{Discussion}

\subsubsection{Conformational dynamics associated with $\mathrm{Ca}^{2+}$ binding at EF-hand 3 and/or 4 modulates DREAM and DNA interaction}

In this report, I have studied functional properties of individual EF-hands of DREAM in regulating the protein conformational dynamics and interaction with DNA. In the previous study, I have shown that $\mathrm{Ca}^{2+}$ association to the EF-hands 3 and 4 in the $\mathrm{C}$ terminal domain of DREAM leads to the changes in conformational dynamics of residue W169. In the $\mathrm{Ca}^{2+}$ bound DREAM, W169 is located in a less polar environment, as demonstrated by a decrease in the emission intensity (-18\%) along with a concomitant hypsochromic shift of $5 \mathrm{~nm}$ of the emission spectra relative to that of $\mathrm{Ca}^{2+}$ free forms (Pham et al., 2015). In addition, the Gaussian distribution component of W169 lifetime $\left(\tau_{1}\right)$ in ApoDREAM decreases from $3.5 \mathrm{~ns}$ to $1.7 \mathrm{~ns}$ in the $\mathrm{Ca}^{2+}$ bound form suggesting lower heterogeneity of the $\mathrm{Ca} 2+$ bound protein Furthermore, the exponential decay fraction of ApoDREAM $\left(f_{1}=77 \%\right)$ significantly decreases when $\mathrm{Ca}^{2+}$ is bound $\left(f_{1}=44 \%\right)$ (Pham et al., 2015).

The overall emission properties of $\mathrm{Ca}^{2+}$ free and $\mathrm{Ca}^{2+}$ bound DREAM-D150N are very similar to those determined for DREAM wild-type. However, closer inspection of the results reveals a small deviation in fluorescence properties of W169 in this mutant, namely, quenching of W169 emission intensity in this mutant is less efficient than in the DREAM wild-type and the Gaussian component of the W169 lifetime is shorter (2.9 ns) in the $\mathrm{Ca}^{2+}$ free form of the protein. In the $\mathrm{Ca}^{2+}$ bound form of protein, residue D150 is located $\sim 14.5$ $\AA$ from W169, indicating that the charge distribution in the EF-hand 2 impacts the conformation of residues located at the interface of the $\mathrm{C}$ - and $\mathrm{N}$ - terminus domains. 
Furthermore, our ITC data show that the binding affinity of ApoDREAM-D150N with the duplex DNA at the high affinity site is weaker $\left(\mathrm{K}_{\mathrm{d} 1}=2.8 \mu \mathrm{M}\right)$ and the change of enthalpy $\left(\Delta \mathrm{H}_{1}=+12 \mathrm{kcal} / \mathrm{mol}\right)$ is smaller compared to that of ApoDREAM $\left(\mathrm{K}_{\mathrm{d} 1}=0.8 \mu \mathrm{M}\right.$ and $\Delta \mathrm{H}_{1}=+35 \mathrm{kcal} / \mathrm{mol}$ ) suggesting a small deviation in the conformational dynamics between the structures of ApoDREAM-D150N and ApoDREAM impact DREAM interactions with DNA.

In order to explain the deviation between the Apo forms of DREAM wild-type and DREAM-D150N mutant, the MD simulation structure of ApoDREAM was superposed with the MD simulation structure of ApoDREAM-D150N (Figure 7.26, left panel).
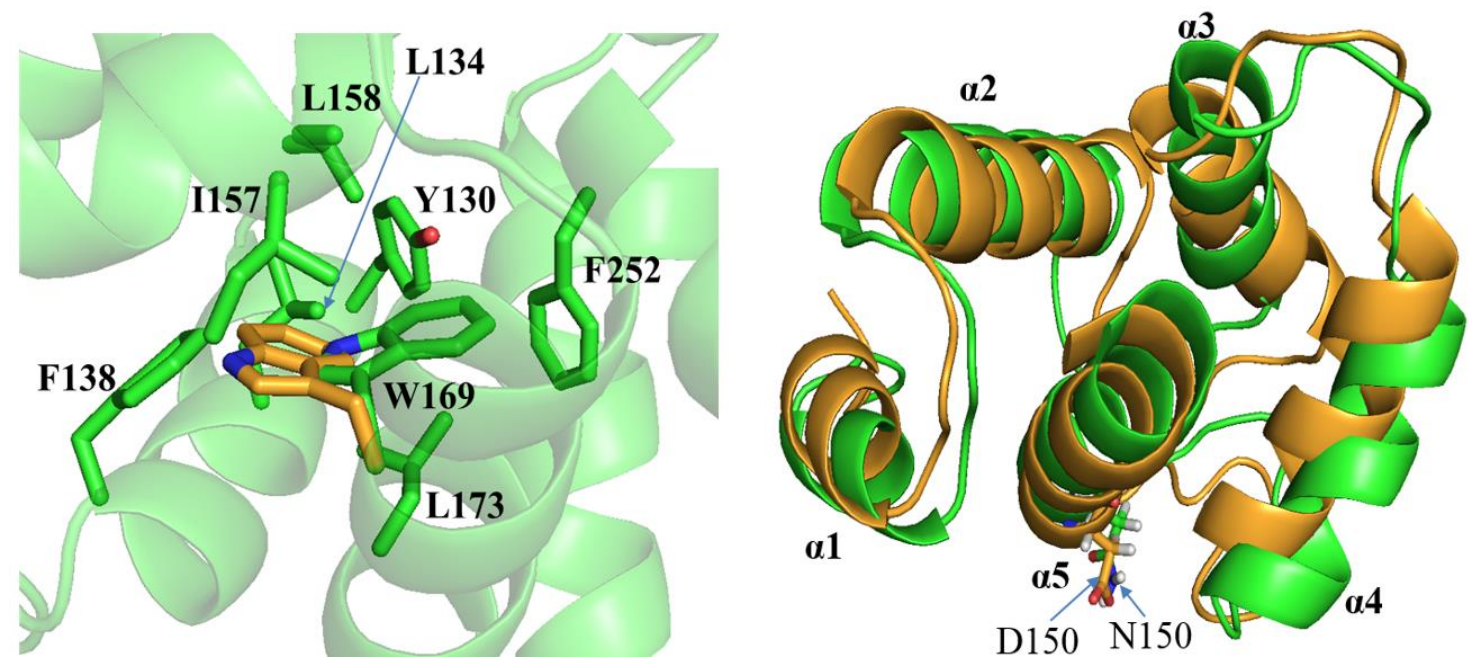

Figure 7.26: Left: Residue W169 is buried in a more hydrophobic environment observed in ApoDREAM (yellow) compared to that obtained in ApoDREAM-D150N mutant (green). Right: The superposition of five $\alpha$-helices in the $\mathrm{N}$-terminal domain of ApoDREAM (yellow) and ApoDREAM-D150N (green).

Interestingly, the indole ring of W169 in ApoDREAM-D150N (green in Figure 7.26, right panel) becomes more buried in the hydrophobic cavity formed by residues Y130, L134, F138, I157, L158, L173, and F252 (Figure 7.26, left panel) compared to that observed in ApoDREAM wild-type. The subtle structural rearrangement of the $\alpha 1$ in the 
N-terminal domain of ApoDREAM-D150 (Figure 7.26, right panel) relative to ApoDREAM explaining the weaker interaction of duplex DNA with ApoDREAM-D150N.

The $\mathrm{Ca}^{2+}$ association at EF-hands 3 and 4 in DREAM-D150N completely eliminates the interaction with duplex DNA with a $\mathrm{K}_{\mathrm{d}}$ of $225 \mu \mathrm{M}$, similar to that of $\mathrm{Ca}^{2+}$ DREAM wild-type (Table 7.3).

The fluorescence properties of W169 are differently modulated by $\mathrm{Ca}^{2+}$ binding to EF-hands 3 and 4. The fluorescence properties of DREAM-E186Q with $\mathrm{Ca}^{2+}$ bound to EF-hand 4 are similar to the fluorescence properties of ApoDREAM, whereas the fluorescence properties of DREAM-E234Q with $\mathrm{Ca}^{2+}$ bound to EF-hand 3 are analogous to emission properties of calcium saturated DREAM wild-type. These data strongly indicate that $\mathrm{Ca}^{2+}$ association to the EF-hand 3 is necessary for structural transition between the ApoDREAM and $\mathrm{Ca}^{2+}$ bound DREAM conformation. Interestingly, the emission data of 1,8-ANS indicate that the accessibility of the C-terminal hydrophobic cavity increases upon $\mathrm{Ca}^{2+}$ binding to EF-hand 3 or $\mathrm{EF}$ hand 4 . On the other hand, the $\mathrm{Ca}^{2+}$ association to either EF-hand 3 or 4 is able to diminish the DREAM affinity for DNA. However, binding of $\mathrm{Ca}^{2+}$ at both EF-hands 3 and 4 is necessary for the regulation of DREAM association to DNA. Also, $\mathrm{Ca}^{2+}$ binding to EF-hand 3 may triggers structural changes that are localized in the vicinity of EF-hand 3 and $\mathrm{Ca}^{2+}$ association to both EF-hands may be necessary for global structural transition in DREAM that is required for inhibition of DREAM interactions with DNA. These data also indicate that fluorescence emission and lifetime of W169 may not be an ideal probe to monitor the global transition in DREAM protein and thus there is a need for additional fluorescence probe to be placed in the $\mathrm{N}$ - terminal domain that would be sensitive to conformational changes in this domain. There are two tyrosine 
residues located in the $\mathrm{N}$ - terminal domain of DREAM, but their emission is efficiently quenched by W169 and they cannot be used for probing conformational changes in the Nterminus domain.

The ITC data show several DNA binding sites on DREAM molecules with one high affinity site and additional three lower affinity site. $\mathrm{Ca}^{2+}$ association to DREAM inhibits DNA binding to both the high and low affinity sites. To understand how $\mathrm{Ca}^{2+}$ binding to DREAM antagonistically modulates the interaction with duplex DNA, the structure of $\mathrm{Ca}^{2+}$ DREAM was superposed with the MD simulation structure of ApoDREAM (Figure 7.27, top panel).

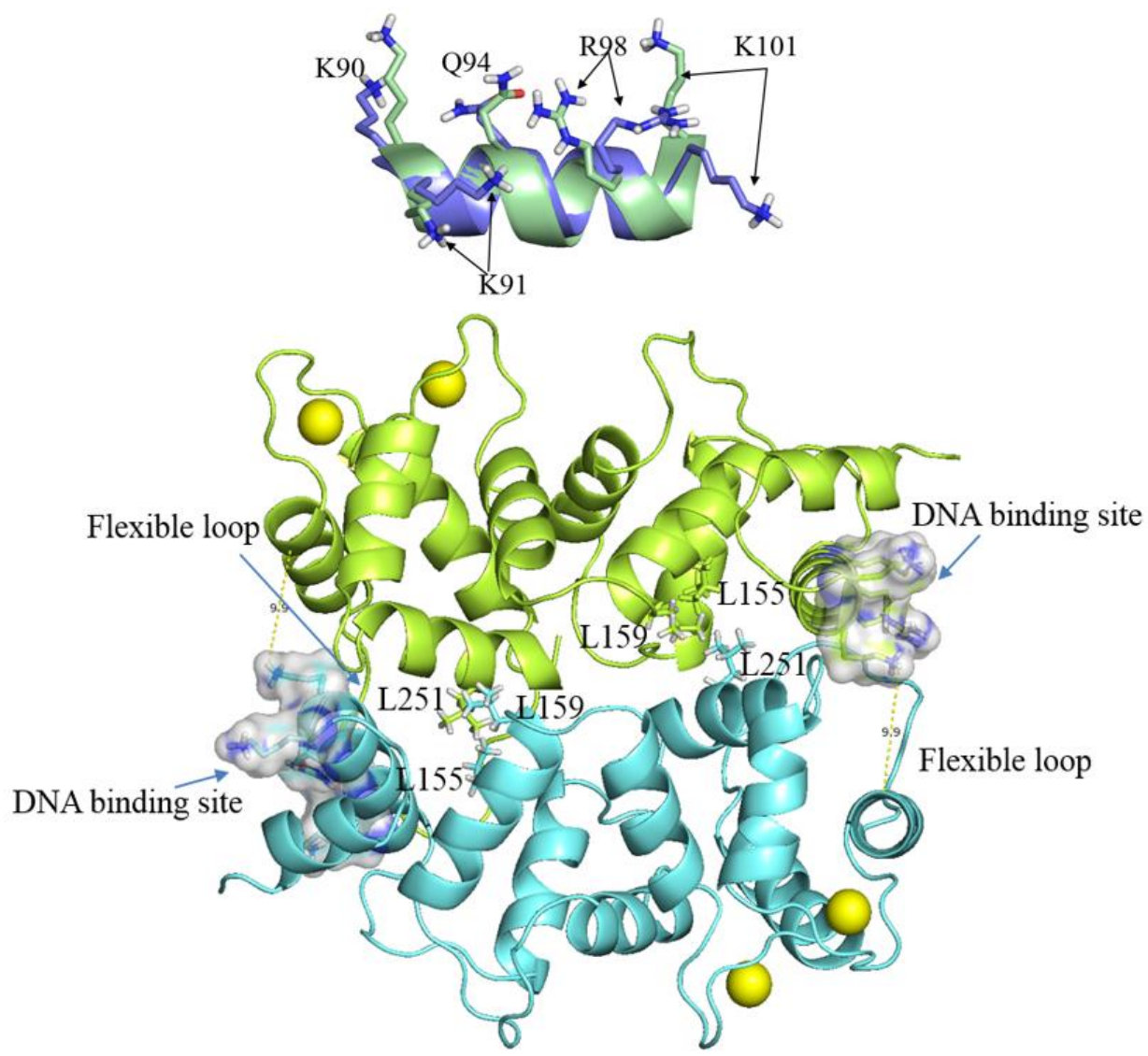

Figure 7.27: Top: Amino acid sequence alignment of residues (K90 - N102) in $\alpha 2$ of ApoDREAM (green) and $\mathrm{Ca}^{2+}$ DREAM (blue). Bottom: The dimer $\mathrm{Ca}^{2+} \mathrm{DREAM}$ was constructed according to the model proposed by Lusin et at. (Lusin et al., 2008). 
The amino acid alignment of residues (K90 - N102) from $\alpha 2$ of ApoDREAM and $\mathrm{Ca}^{2+}$ DREAM (Figure 7.27, top panel) reveals that the side chains of residues in $\alpha 2$ (Q94, $\mathrm{R} 98, \mathrm{~K} 101)$ in $\mathrm{Ca}^{2+} \mathrm{DREAM}$ rotate about 180 degrees, preventing formation of DREAMDNA complex. In addition, the structure of head-to-tail homodimer of $\mathrm{Ca}^{2+} \mathrm{DREAM}$ (Figure 7.27, bottom panel), constructed using the dimeric model proposed by Lusin et al (Lusin et al., 2008), reveals the loop connecting $\alpha 7$ and $\alpha 8$ from the second $\mathrm{Ca}^{2+}$ DREAM molecule blocks the DNA binding interface $(\alpha 2)$ in the first $\mathrm{Ca}^{2+}$ DREAM molecule which prevents interaction of DNA.

\subsubsection{Association of $\mathrm{Mg}^{2+}$ at EF-hand 2 structurally facilitates the interaction between DREAM and DNA}

Because the concentration of free $\mathrm{Mg}^{2+}$ is maintained at nearly constant level of 1 $\mu \mathrm{M}$ in cells, $\mathrm{Mg}^{2+}$ is not likely to directly regulate cell signaling (Romani, Scarpa 1992). However, DREAM possesses a highly $\mathrm{Mg}^{2+}$ sensitive EF-hand $2\left(\mathrm{~K}_{\mathrm{d}} \sim 13 \mu \mathrm{M}\right)$ that raises a question about the physiologically relevance of $\mathrm{Mg}^{2+}$ DREAM in the resting state of the cell, as well as its role in modulating the interaction with DNA. The functional role of $\mathrm{Mg}^{2+}$ bound to EF-hand 2 in DREAM in modulating DNA interaction was demonstrated by experimental data. Dynamical network analysis illustrates that $\mathrm{Mg}^{2+}$ binding at EF-hand 2 alters the orientation of $\alpha 2$ in such way that the residues L93, L96, and F100 on the inner side of $\alpha 2$ are coupled with the hydrophobic residues F121 (from $\alpha 3$ ), F148, F151, V151, L155, and L159 from $\alpha 5$ (exiting helix of EF-hand 2), while side chain of the basic and polar residues K90, K91, Q94, S95, R98, K101 at DNA binding interface are extruded to the solvent (Figure 7.20). Our ITC data show that affinity for DNA specifically binding to $\mathrm{Mg}^{2+} \operatorname{DREAM}\left(\mathrm{K}_{\mathrm{d} 1}=0.3 \mu \mathrm{M}\right)$ is somewhat higher compared to that of ApoDREAM $\left(\mathrm{K}_{\mathrm{d} 1}\right.$ 
$=0.8 \mu \mathrm{M})$. In addition, association of DNA to $\mathrm{Mg}^{2+}$ DREAM is an exothermic reaction with favorable enthalpy $(\Delta \mathrm{H})$ of $-18 \mathrm{kcal} / \mathrm{mol}$, whereas binding of DNA to ApoDREAM is an endothermic reaction with $\Delta \mathrm{H}$ of $+35 \mathrm{kcal} / \mathrm{mol}$. These results suggest that the binding mechanisms are different for association of DNA to Apo and $\mathrm{Mg}^{2+}$ forms of DREAM. To further examine the different mechanisms of DNA association with ApoDREAM and $\mathrm{Mg}^{2+}$ DREAM, time-resolved anisotropy experiments were carried out. Our anisotropy decay data illustrate that DNA binds to tetrameric ApoDREAM and monomeric $\mathrm{Mg}^{2+}$ DREAM. The binding interface between ApoDREAM and the duplex DNA was determined using experimental restraints from previous studies (Carrion, Link et al. 1999a, Ledo, Link et al. 2000, Lusin, Vanarotti et al. 2008) for the input in protein-DNA computational docking. The model of $\mathrm{Mg}^{2+}$ DREAM-DNA complex was not investigated in this study due to the lack of experimental information for docking input. These results demonstrate that assembly of ApoDREAM and DNA involves two major types of interaction: electrostatic interactions between the negatively charged phosphate oxygens from nucleotides C43, A13, and C41 from DNA and the positively charged residues K90, K91, and K101 from $\alpha 2$ in DREAM. The complex is further stabilized by hydrogen bond interactions between pairs of amino acid residue-nucleotide: K90-C43, K91-A13, K101A41, Q94-T42, S95-A13, and Q120-G15 (Figure 7.25). Furthermore, the complex is further stabilized by hydrogen bonds and cation- $\pi$ interactions between the side chain of residue R98 from DREAM with the nucleotides at the minor groove of the target DRE site formed by $5{ }^{\prime}{ }^{10} \mathrm{GTCA}^{13}-3$ ' and ' $3{ }_{-}^{41} \mathrm{CAGT}^{38}-5$ '. The residue R98 in DREAM plays a role in the interaction not only with DNA (Lusin et al., 2008), but also with $\mathrm{K}^{+}$channel $\mathrm{K}_{\mathrm{v}} 4.3$ 
(Gonzalez, Pham et al. 2014), highlighting its biological relevance in modulating interactions with DNA and target proteins.

\section{4 Summary}

In this report, we show comprehensive evidence demonstrating the impact of $\mathrm{Mg}^{2+} / \mathrm{Ca}^{2+}$ on conformational dynamics of DREAM as well as the functional properties of individual EF-hands in DREAM upon interaction with duplex DNA. We demonstrate that $\mathrm{Mg}^{2+}$ binding at EF-hand 2 structurally stabilizes the DREAM N-terminal domain, which facilitates interaction with duplex DNA. The binding of $\mathrm{Ca}^{2+}$ at either EF-hand 4 is able to modulate the interaction of DNA. However, binding of $\mathrm{Ca}^{2+}$ at both EF-hands 3 and 4 is necessary to abolish interaction between DREAM and DNA. These findings provide insight into the roles of individual EF-hands in DREAM under physiological and pathological conditions, which can be used as a model to uncover the functional properties of EF-hands in other neuronal calcium sensor proteins and to characterize the effects of $\mathrm{Mg}^{2+}$ on various $\mathrm{Ca}^{2+}$ signaling pathways in cells. 


\section{CHARACTERIZE KINETICS AND THERMODYNAMICS ASSOCIATED}

WITH CARBON MONOXIDE BINDING OR RELEASING FROM HORSE

HEART MYOGLOBIN AND MICROPEROXIDASE-11 IN SODIUM BIS-(2-

ETHYLHEXYL) SULFOSUCCINATE ENCAPSULATION: A MODEL SYSTEM

TO STUDY CONFORMATIONAL DYNAMICS OF DREAM FULL LENGTH IN

MEMBRANE ANCHORED STATE.

(The work described in this chapter was partially published in (Larsen, Vetromile et al. 2011))

\subsection{Background and significance}

Reverse (inverted) micelles (RM) are nanoscale droplets of water surrounded by amphiphilic surfactants in nonpolar solvent with surfactant molecules oriented in such way that the nonpolar tails are in contact with the organic phase and the polar heads enclose a water pool inside the micellar cavity (Figure 8.1).

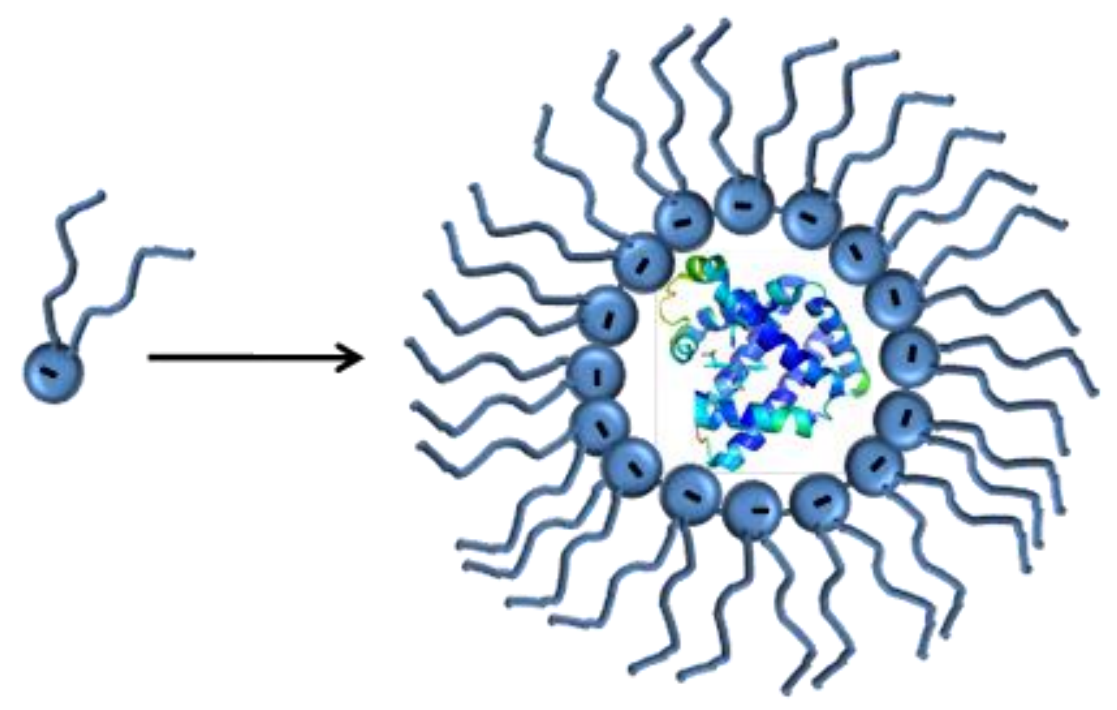

Figure 8.1: The schematic presentation of AOT molecule (left) and a reverse micelle particle containing an $\mathrm{Mb}$ molecule encapsulated within internal water pool (right). 
Reverse micelles have attracted considerable attention as micromedia for chemical and biochemical reactions due to their ability to solubilize hydrophilic reactants in organic solvents. Reverse micelles based upon sodium bis-(2-ethylhexyl) sulfosuccinate (AOT) have been extensively studied using various physical techniques since these micelles are thermodynamically stable, optically transparent and the size of the internal water pool can be varied from $10 \AA$ ( $\approx 30$ water molecules) to $140 \AA$ ( $\approx 400,000$ water molecules) through varying the molar water to surfactant ratio (wo $=[$ water $] /[$ surfactant $]$ ) (Kotlarchyk et al., 1985; Moilanen et al., 2007; Fenn et al., 2009). The properties of encapsulated water molecules are traditionally described using a two-state model as originally proposed by Zinsli (Zinsli, 1979). Interfacial water molecules that solubilize charged surfactant head groups as well as counter cations exhibit increased viscosity and slow relaxation rates whereas the water molecules located within the core of the water pool show properties similar to bulk water. Reverse micelles have been extensively used to probe the effect of confinement and hydration on protein structure, dynamics, and folding. The structural properties of encapsulated proteins depend on the water pool size, surfactant charge and interactions between protein and surfactant (Van Horn et al., 2005; Van Horn et al., 2009; Naoe et al., 2004). For example, it has been shown that encapsulation of cytochrome $\mathrm{c}$ in AOT RM is associated with a structural destabilization, opening of the heme crevice and dissociation of the Met- 80 heme axial ligand (Abel et al., 2010; Brochette et al., 1988). To investigate the conformational dynamics in restricted environment of RM, I have used PAC and transient absorption to characterize the kinetics and thermodynamics associated with $\mathrm{CO}$ binding/releasing from horse heart Mb and from microperoxidase-11 (MP-11). Protein MP-11 is an 11 residue polypeptide fragment obtained by proteolytic cleavage of 
cytochrome c. The heme group in MP-11 is covalently attached to the peptide chain through two thioether linkages and retains axial histidine ligand (Verbaro et al., 2009).

\subsection{Results}

\subsubsection{UV/Vis absorption spectra}

The absorption spectra of ferric, ferrous and $\mathrm{CO}$ bound $\mathrm{Mb}$ encapsulated in AOT $\mathrm{RM}$ of $\mathrm{w}_{\mathrm{o}}=40$ are displayed in Figure 8.2. The optical spectrum of $\mathrm{Fe}^{3+} \mathrm{Mb}$ exhibits a maximum of the Soret band at $414 \mathrm{~nm}$ and a broader absorption band with maximum at $534 \mathrm{~nm}$ with an additional shoulder at $564 \mathrm{~nm}$ (Table 8.1). These values are very similar to the absorption spectrum of a hemichrome $\mathrm{Mb}$ intermediate with bis-histidine heme iron coordination (Miksovksa et al., 2006). Formation of the hemichrome Mb was previously reported for metMb exposed to low concentration of $\mathrm{GuHCl}$ (Culbertson and Olson, 2010) or to $\mathrm{Fe}^{3+} \mathrm{Mb}$ complexed with negatively charged surfactants such as sodium dodecyl sulfate (Miksovksa et al., 2006) or AOT (Murakami et al., 2011).
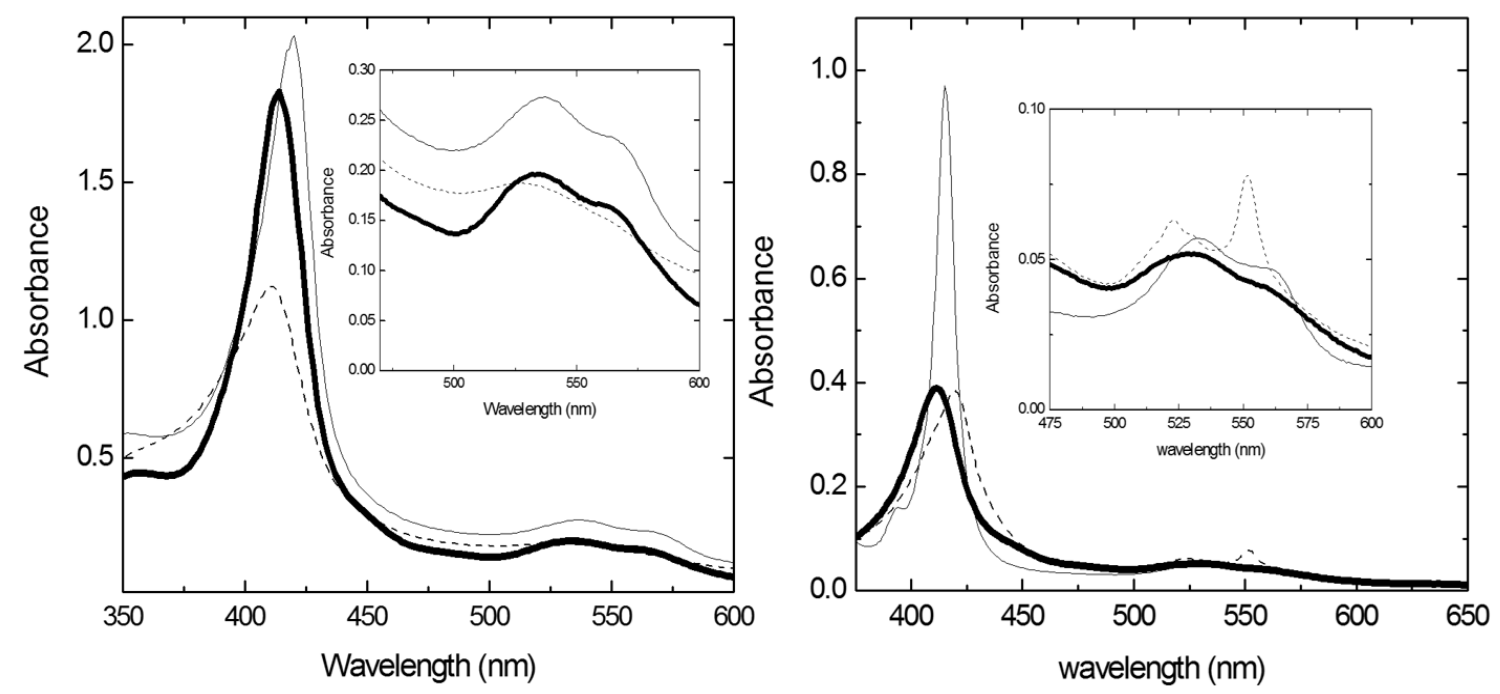

Figure 8.2: Optical absorption spectra of Mb (left) and MP-11 (right) encapsulated within $\mathrm{RM} \mathrm{w}=40$. The traces for $\mathrm{Fe}^{3+} \mathrm{Mb} / \mathrm{Fe}^{3+} \mathrm{Mp}-11$ are shown as solid thick lines, for $\mathrm{Fe}^{2+} \mathrm{Mb} / \mathrm{Fe}^{2+} \mathrm{MP}-11$ as dashed lines and for CO-Mb and CO-MP11 as solid thin lines. 
Reduction of Mb in AOT micelles is associated with a hypsochromic shift of the Soret band to $411 \mathrm{~nm}$ and the appearance of a broad band in the visible region, $\lambda_{\max }=626$ $\mathrm{nm}$. Such a large blue shift in the Soret band absorption maxima relative to $\mathrm{Fe}^{2+} \mathrm{Mb}$ in aqueous solutions $\left(\lambda_{\max }=434 \mathrm{~nm}\right)$ indicates pronounced changes in the heme binding pocket.

Table 8.1: The position of the Soret band and Q bands for Mb, and MP-11 encapsulated within the AOT reverse micelles, $\mathrm{w}_{\mathrm{o}}=40$. The numbers is parenthesis corresponds to the $\lambda_{\max }$ for $\mathrm{Mb}$ and $\mathrm{MP}-11$ in aqueous solutions, $\mathrm{pH}=7.0$.

\begin{tabular}{|l|c|l|l|c|l|}
\hline & $\begin{array}{c}\text { Soret band } \\
\lambda_{\max }(\mathrm{nm})\end{array}$ & $\begin{array}{c}\text { Q bands } \\
\lambda_{\max }(\mathrm{nm})\end{array}$ & & $\begin{array}{c}\text { Soret band } \\
\lambda_{\max }(\mathrm{nm})\end{array}$ & $\begin{array}{c}\text { Q bands } \\
\lambda_{\max }(\mathrm{nm})\end{array}$ \\
\hline $\mathrm{Fe}^{3+} \mathrm{Mb}$ & $\begin{array}{c}414 \\
(406)\end{array}$ & $\begin{array}{l}534,565 \\
(508.630)\end{array}$ & $\mathrm{Fe}^{3+} \mathrm{MP}-11$ & $\begin{array}{c}410 \\
(400)\end{array}$ & $\begin{array}{l}521 \\
(532)\end{array}$ \\
\hline $\mathrm{Fe}^{2+} \mathrm{Mb}$ & $\begin{array}{c}411 \\
(435)\end{array}$ & $\begin{array}{l}531 \\
(560)\end{array}$ & $\mathrm{Fe}^{2+} \mathrm{MP}-11$ & $\begin{array}{c}419 \\
(416)\end{array}$ & $\begin{array}{l}523,551 \\
(520,550)\end{array}$ \\
\hline $\mathrm{CO}-\mathrm{Mb}$ & $\begin{array}{c}420 \\
(424)\end{array}$ & $\begin{array}{l}537,567 \\
(540,579)\end{array}$ & $\mathrm{CO}-\mathrm{MP}-11$ & $\begin{array}{c}415 \\
(412)\end{array}$ & $\begin{array}{l}528,562 \\
(525)\end{array}$ \\
\hline
\end{tabular}

The absorption spectra of MP-11 in RM are shown in Figure 8.2 (right). The Soret band of $\mathrm{Fe}^{3+} \mathrm{MP}-11$ is centered at $410 \mathrm{~nm}$ and upon heme iron reduction, shifts to $419 \mathrm{~nm}$ with two $\mathrm{Q}$ bands appearing at $523 \mathrm{~nm}$ and $551 \mathrm{~nm}$ that are characteristic of a low-spin sixcoordinated heme $c$ species. The formation of six-coordinate species was previously observed for MP-11 solubilized in aqueous solutions and was attributed to intermolecular coordination between the heme iron and $\alpha-\mathrm{NH}_{2}$ group of Val11 or $\varepsilon-\mathrm{NH}_{2}$ group of Lys 13 (Wang, Van Wart 1989)(144). The optical absorption results suggest that the intermolecular coordination of MP-11 is retained upon peptide encapsulation within the reverse micelles. Saturation of $\mathrm{Fe}^{2+} \mathrm{MP}-11$ within $\mathrm{RM}$ with $\mathrm{CO}$ leads to the formation of the six-coordinated low-spin heme iron. Interestingly, the Soret band maxima of MP-11 adducts are red-shifted relative to the optical spectra of MP-11 in aqueous solutions 
suggesting strong interactions between MP-11 and the negatively charged sulfate groups of the AOT molecules.

\subsubsection{Photoacoustic calorimetry results}

The PAC trace for $\mathrm{CO}$ photo-dissociation from $\mathrm{Mb}$ in $\mathrm{RM}$ is displayed in Figure 8.2 together with the PAC trace for $\mathrm{Fe}^{3+} \mathrm{Mb}$ (used as the calorimetric reference). The sample acoustic wave is shifted in phase relative to the reference indicating the presence of volume/enthalpy changes occurring on timescales from $\sim 50$ ns to $\sim 15 \mu$ s. These data indicate that $\mathrm{CO}$ escape from $\mathrm{Fe}^{2+} \mathrm{Mb}$ in $\mathrm{RM}$ can be described as a two-step process with the prompt step $\left(\tau_{\text {prompt }}<50 \mathrm{~ns}\right)$ corresponding to Fe-CO bond cleavage and the subsequent step reflecting $\mathrm{CO}$ escape from the protein matrix to the surrounding solvent.

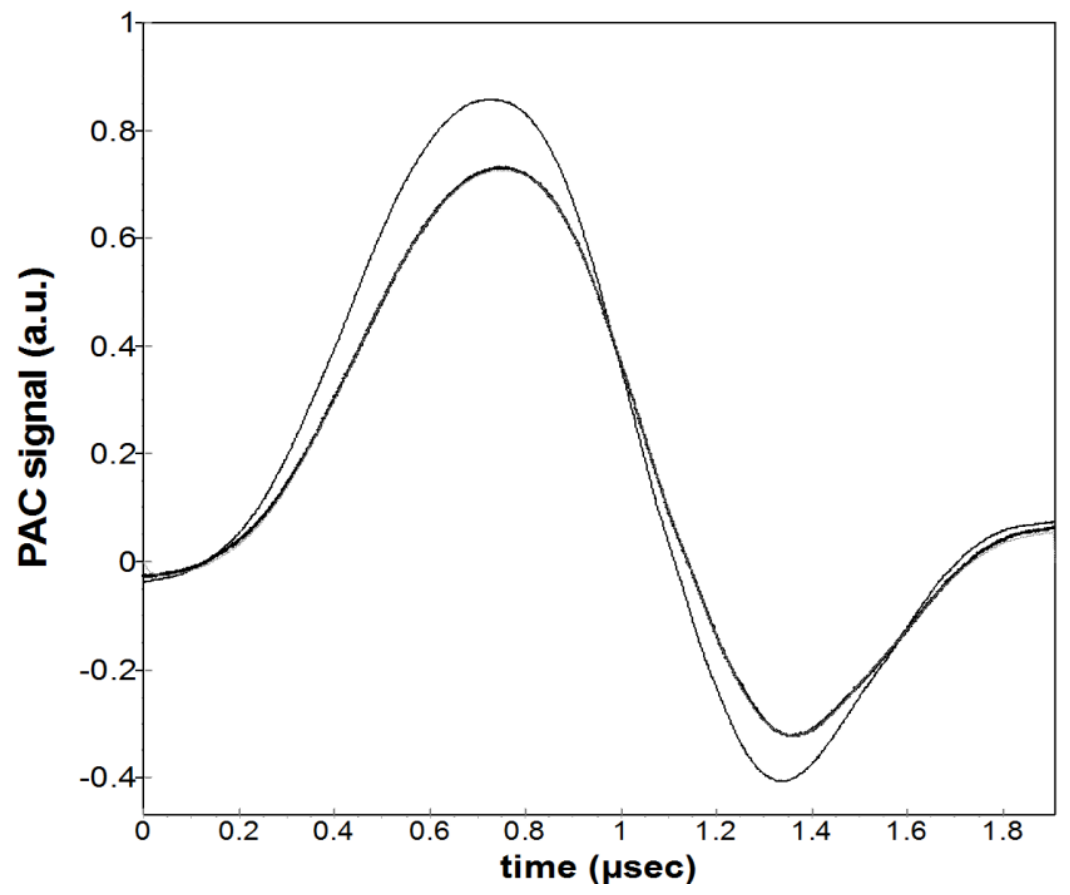

Figure 8.3: PAC traces for $\mathrm{CO}$ photo-dissociation from $\mathrm{Fe}^{2+} \mathrm{Mb}$ in $\mathrm{AOT} \mathrm{RM}$ of $\mathrm{w}_{\mathrm{o}}=40$ (thick black line), for the reference compound $\left(\mathrm{Fe}^{3+} \mathrm{Mb}\right.$ in AOT RM of $\left.\mathrm{w}_{\mathrm{o}}=40\right)$ and the fit (gray line). 
The PAC sample and reference traces were measured over the temperature range from 20 to $35{ }^{\circ} \mathrm{C}$ and deconvolution of the PAC traces provided the rate constant for $\mathrm{CO}$ escape from $\mathrm{Fe}^{2+} \mathrm{Mb}$ encapsulated within AOT RM to be $\sim 8.3 \times 10^{6} \mathrm{~s}^{-1}$ at $20^{\circ} \mathrm{C}$ with an associated activation energy, $E_{a}=24 \pm 13 \mathrm{~kJ} \mathrm{~mol}^{-1}$. The observed rate constant is approximately six times faster relative to the rate constant for $\mathrm{CO}$ escape from $\mathrm{Fe}^{2+} \mathrm{Mb}$ in aqueous solutions at $\mathrm{pH} 7.0\left(\mathrm{k}_{\mathrm{esc}}=1.4 \times 10^{6} \mathrm{~s}^{-1}\right.$ and $\left.\mathrm{E}_{\mathrm{a}}=43 \pm 3 \mathrm{~kJ} \mathrm{~mol}^{-1}\right)($ Belogortseva et al., 2007). Based upon spectroscopic data and molecular dynamics approaches, a comprehensive molecular mechanism for ligand migration in $\mathrm{Mb}$ was proposed including the initial diffusion of the photo-dissociated ligand into the internal network of hydrophobic cavities and subsequent ligand return into the distal heme binding pocket (Small et al., 1992; Mouawad et al., 2005). The escape of CO from the protein matrix requires opening of a transient channel between the heme propionates through an outward rotation of the distal histidine side chain and the rate for closing/opening of histidine gate was estimated to occur on timescale of $1-10 \mu$ s, which is comparable to the $700 \mathrm{~ns}$ time constant determined in previous PAC measurements (Larsen et al., 2011). The faster ligand escape observed for $\mathrm{Mb}$ within $\mathrm{RM}$ indicates that either Mb confinement within RM and/or electrostatic interactions between the encapsulated $\mathrm{Mb}$ and negatively charged micellar interface accelerates the transition between the closed and open conformations of the histidine gate resulting in the faster $\mathrm{CO}$ exit from protein matrix. Alternatively, perturbations to the $\mathrm{Mb}$ tertiary structure may trigger the opening of new ligand migration pathway(s) with lower activation barriers that are not populated under native conditions. 


\subsubsection{Transient absorption results}

To further investigate the interactions between $\mathrm{CO}$ and $\mathrm{Fe}^{2+} \mathrm{Mb} / \mathrm{Fe}^{2+} \mathrm{MP}-11$ in reverse micelles, the rate constants for $\mathrm{CO}$ rebinding were determined using transient absorption spectroscopy (Figure 8.4). Rebinding of $\mathrm{CO}$ to the heme iron in the $\mathrm{Fe}^{2+} \mathrm{Mb}$ AOT system is a biphasic process with the faster process occurring with the time constant of $41 \mu \mathrm{s}\left(\mathrm{A}_{1} \sim 0.7\right)$ and the second phase having a lifetime of $176 \mu \mathrm{s}\left(\mathrm{A}_{2} \sim 0.3\right)$ at $20{ }^{\circ} \mathrm{C}$. $\mathrm{A}$ similar biphasic process was observed for CO rebinding to MP-11 with $\tau_{1}=23 \mu$ s and $\tau_{2}$ $=57 \mu$ s with equal amplitudes for both phases (data not shown). The rate constants for $\mathrm{CO}$ rebinding to $\mathrm{Fe}^{2+} \mathrm{Mb}$ were probed over the temperature range from $15{ }^{\circ} \mathrm{C}$ and $30{ }^{\circ} \mathrm{C}$ to determine the activation barrier for ligand rebinding (Figure 8.4, Inset).

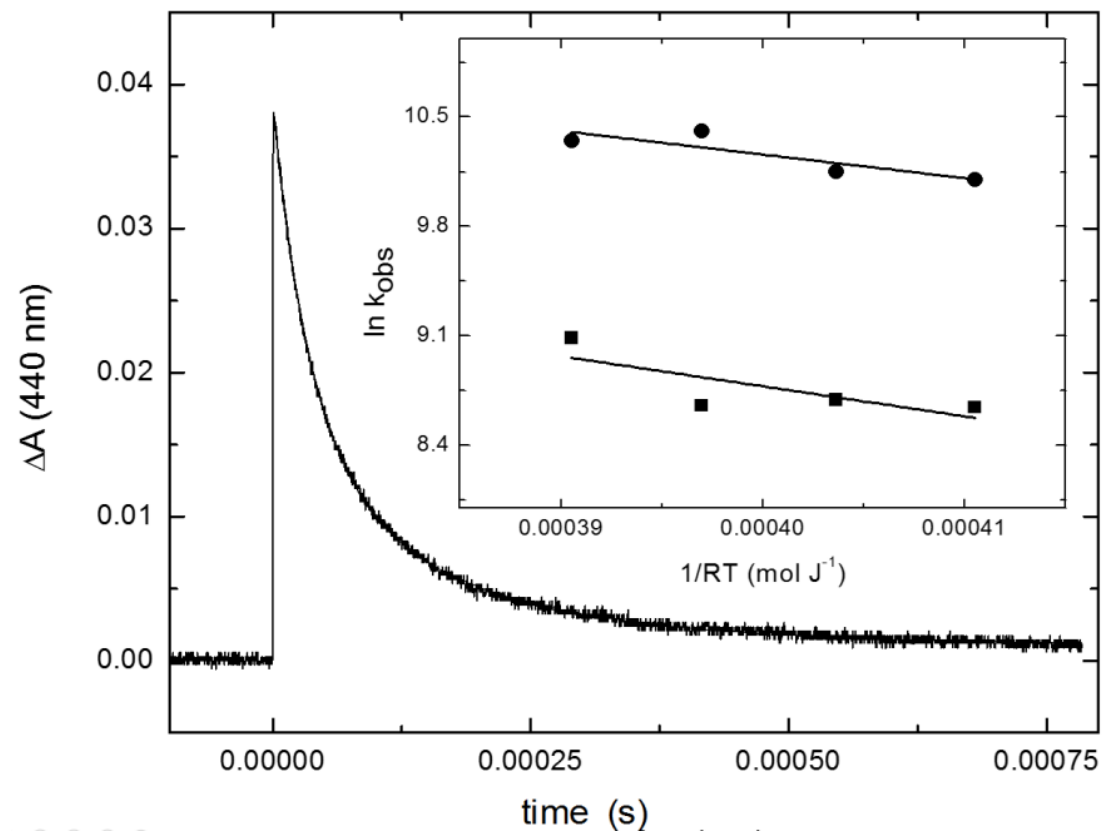

Figure 8.4: Transient absorption trace for $\mathrm{CO}$ rebinding to $\mathrm{Fe}^{2+} \mathrm{Mb}$ encapsulated within $\mathrm{RM}, \mathrm{w}_{\mathrm{o}}=40$. Inset: Arrhenius plot of the rate constants for the fast (circles) and slow (squares) phase of the $\mathrm{CO}$ rebinding to $\mathrm{Mb}$. 
The fast phase shows an activation barrier of $15 \pm 6 \mathrm{~kJ} \mathrm{~mol}^{-1}$ and similar activation barrier was determined for the slow phase, $\mathrm{E}_{\mathrm{a}}=19 \pm 9 \mathrm{~kJ} \mathrm{~mol}^{-1}$. These values are comparable to the activation energies determined for $\mathrm{CO}$ rebinding to $\mathrm{Fe}^{2+} \mathrm{Mb}$ in aqueous solutions, $\mathrm{E}_{\mathrm{a}} \sim 19 \mathrm{~kJ} \mathrm{~mol}^{-1}$ (Hasinoff, 1974).

Overall, these data are in agreement with the photoacoustic results confirming that Mb encapsulation within RM leads to the opening of the protein structure facilitating ligand access to the heme binding pocket. The presence of bi-exponential $\mathrm{CO}$ rebinding kinetics to both $\mathrm{Fe}^{2+} \mathrm{Mb}$ and $\mathrm{Fe}^{2+} \mathrm{MP}-11$ is somewhat surprising. Analysis of the $\mathrm{CO}$ rebinding data in $\mathrm{RM}$ is complicated as the $\mathrm{CO}$ concentration within the micellar water pool and surfactant interface is unknown. However, the presence of the multi-exponential $\mathrm{CO}$ rebinding kinetics points out that $\mathrm{Fe}^{2+} \mathrm{MP}-11$ and $\mathrm{Fe}^{2+} \mathrm{Mb}$ may be located at least in two distinct environments, such as water pool and surfactant interface, with different $\mathrm{CO}$ concentration or that the $\mathrm{CO}$ rebinds from both the interior water cavities as well as diffusion from the bulk organic solvent.

\subsection{Summary and Outlook}

The ability to probe thermodynamics associated with physiological processes in biomolecules on sub-millisecond time scales remains a significant challenge in molecular biophysics. Nonetheless, as demonstrated in the sections above, time resolved photothermal methods offer a unique opportunity to access enthalpy and molar volume changes associated with photo-initiated processes on time scales ranging from tens of milliseconds down to tens of nanoseconds. Photoacoustic calorimetry has now been widely applied to a vast array of biological molecules with photo-triggerable physiological processes and thermodynamic profiles have been revealed on very fast time scales. I have 
now demonstrated the utility of the PAC method for proteins encapsulated within reverse micelles and are currently employing these methods to investigate the conformational dynamics of DREAM full length (residues 1 -256) in membrane-anchored state as well as the interaction of presenilins with DREAM full-length encapsulated in reverse micelles upon photolysis of caged $\mathrm{Ca}^{2+}$ compound. 


\section{CONCLUSIONS}

The conformational dynamics and stability associated with $\mathrm{Mg}^{2+}$ and/or $\mathrm{Ca}^{2+}$ binding to DREAM in the regulation of interactions between DREAM and DNA or presenilins have been successfully delineated throughout this dissertation.

The impact of $\mathrm{Ca}^{2+}$ and/or $\mathrm{Mg}^{2+}$ on conformational dynamics and stability of DREAM have been described in Chapters 4 and 5. The binding of $\mathrm{Ca}^{2+}$ to DREAM triggers the reorganization of the interface between EF-hand 2 and EF-hand 3 with the W169 side chain being located in a less polar environment. In addition, association of $\mathrm{Ca}^{2+}$ reduces solvent accessibility to the W169 residue due to tertiary structure alterations and/or decreased conformational flexibility. In addition, a transition from the $\mathrm{t}$ to $\mathrm{g}+$ rotamer of W169 side chain was observed during molecular dynamic simulations supporting the idea that the bimodal distribution could arise from two rotameric orientations of W169 with the side chain of the $\mathrm{g}+$ rotamer being more solvent exposed than that of the $\mathrm{t}$ rotamer. The W169 lifetime data indicates that DREAM can adopt an additional conformation with a long W169 lifetime of $7.5 \mathrm{~ns}$ that may reflect conformations of DREAM populated in the presence of lipid membranes and vesicles.

In addition, I have also shown that association of $\mathrm{Mg}^{2+}$ and/or $\mathrm{Ca}^{2+}$ to DREAM EFhands increases the stability of the protein, although to different extents. Despite the low impact of $\mathrm{Mg}^{2+}$ association with EF-hand 2 on the DREAM tertiary structure, the presence of $\mathrm{Mg}^{2+}$ stabilizes the native state with respect to a partially unfolded state. The results show that DREAM unfolding is a complex process that occurs through a partially unfolded, intermediate state. The $\mathrm{Ca}^{2+}$ association to EF-hands 3 and 4 stabilizes the partially unfolded state with respect to the unfolded state whereas the association of $\mathrm{Mg}^{2+}$ increases 
the stability of the native state only in the presence of $\mathrm{Ca}^{2+}$. These results suggest that the intracellular fluctuations in $\mathrm{Ca}^{2+}$ and $\mathrm{Mg}^{2+}$ concentrations control the global conformation and stability of DREAM and likely regulate its interactions with intracellular partners.

In Chapter 6, I have presented that association of presenilin- 1 carboxyl $\alpha$-helix 9 (HL9) with DREAM is $\mathrm{Ca}^{2+}$ dependent. The assembly of DREAM and HL9 is primarily dominated by hydrophobic interactions between residues F462 and F465 from HL9 and residues M191, I194, M197, M198, M249, F252, V255, and I256 at the C-terminal hydrophobic cavity in DREAM. I have also illustrated that residue F252 as well as EFhand 3 in DREAM play crucial roles in the regulation of the interaction of DREAM with HL9. These results provide an insight into the conformational dynamics of DREAM associated with $\mathrm{Ca}^{2+}$ and/or $\mathrm{Mg}^{2+}$ binding to DREAM in regulation of DREAM and presenilins interactions. Our findings also provide the crucial hot spots at the binding interface between DREAM and presenilin 1, which provides the potential druggable pockets for structure-based development of a novel drug compounds to treat Alzheimer's disease.

The functional roles of individual EF-hands in DREAM in modulating the conformational dynamics of DREAM protein and upon interactions with DNA have been delineated in Chapter 7. I have shown that $\mathrm{Mg}^{2+}$ association at EF-hand 2 structurally stabilizes the N-terminal alpha-helices 1, 2, and 5, thereby assisting the interaction with DNA. The binding of $\mathrm{Ca}^{2+}$ at EF-hand 3 induces significant structural changes in DREAM, represented by the critical paths comprised of hydrophobic residues F88, F121, Y130, F133, L134, A137, V152, L155, I157, L159, W169, and L173. These findings illustrate 
the critical role of EF-hand 3 for $\mathrm{Ca}^{2+}$ signal transduction from the carboxy- to aminoterminus in DREAM. Interestingly, $\mathrm{Ca}^{2+}$ association at EF-hand 4 stabilizes the carboxyterminus by forming a strong coupled network consisting of hydrophobic residues L167, F171, I232, F235, and L236 in DREAM.

Lastly, in Chapter 8, I successfully characterize kinetics and thermodynamics associated with carbon monoxide binding or releasing from horse heart myoglobin and microperoxidase-11 in sodium bis-(2-ethylhexyl) sulfosuccinate encapsulation for the development of a novel model system to study conformational dynamics of DREAM full length in membrane anchored state. I have demonstrated that transient absorption and photoacoustic calorimetry methods for proteins encapsulated within reverse micelles are novel and reliable approaches to investigate the conformational dynamics of DREAM full length (residues 1 -256) in membrane-anchored state as well as the interaction of presenilins with DREAM full-length encapsulated in reverse micelles. The study also reveals novel insights into the protein conformation, physiological function, and proteinmembrane interactions. Such information is critical to understanding NCS proteins function within the confined space of the cell as well as to develop a more reliable method to study myristoylation or palmitoylation switch of NCS proteins. 


\section{LIST OF REFERENCES}

Burgoyne, R. and Weiss, J. (2001). The Neuronal Calcium Sensor Family of $\mathrm{Ca}^{2+}$ Binding Proteins.

Abel, S., Waks, M., and Marchi, M. (2010). Molecular Dynamics Simulations of Cytochrome C Unfolding in AOT Reverse Micelles: The First Steps. The European Physical Journal E, 32(4): 399-409.

Alcala, J., Gratton, E., and Prendergast, F. (1987). Interpretation of Fluorescence Decays in Proteins Using Continuous Lifetime Distributions. Biophysical Journal, 51(6): 925-936.

Alexander, W., Eargle, J., and Luthey-Schulten, Z. (2010). Experimental and Computational Determination of tRNA Dynamics. FEBS Letters; Transfer RNA, 584(2): 376-386.

Ames, J., Hendricks, K., Strahl, T., Huttner, I., Hamasaki, N., and Thorner, J. (2000). Structure and Calcium-Binding Properties of Frq1, a Novel Calcium Sensor in the Yeast Saccharomyces Cerevisiae. Biochemistry, 39(40): 12149-12161.

Ames, J., Ishima, R., Tanaka, T., Gordon, J., Stryer, L., and Ikura, M. (1997). Molecular Mechanics of Calcium-Myristoyl Switches. Nature, 389(6647): 198-202.

Ames, J. and Lim, S. (2012). Molecular Structure and Target Recognition of Neuronal Calcium Sensor Proteins. Biochimica et Biophysica Acta, 1820(8): 1205-1213.

Ames, J, Hamasaki, N., and Molchanova, T. (2002). Structure and Calcium-Binding Studies of a Recoverin Mutant (E85Q) in an Allosteric Intermediate State. Biochemistry, 41(18): 5776-5787.

Ames, J., Hendricks, K., Strahl, T., Huttner, I., Hamasaki, N., and Thorner, J. (2000). Structure and Calcium-Binding Properties of Frq1, A Novel Calcium Sensor in the Yeast Saccharomyces cerevisiae. Biochemistry, 39(40):12149-12161.

Ames, J. and Ikura, M. (2006). Molecular Structure and Target Recognition of Neuronal Calcium Sensor Proteins. 317-329.

Ames, J., Ishima, R., Tanaka, T., Gordon, J., Stryer, L., and Ikura, M. (1997). Molecular Mechanics of Calcium-Myristoyl Switches. Nature, 389(6647):198-202.

Ames, J., Levay, K., Wingard, J., Lusin, J., and Slepak, V. (2006). Structural Basis for Calcium-Induced Inhibition of Rhodopsin Kinase by Recoverin. Journal of Biological Chemistry, 281(48): 37237-37245. 
Ames, J. and Lim, S. (2012). Molecular Structure and Target Recognition of Neuronal Calcium Sensor Proteins. Biochimica et Biophysica Acta (BBA) - General Subjects, 1820(8):1205-1213.

An, W., Bowlby, M., Betty, M., Cao, J., Ling, H., Mendoza, G., Hinson, J., Mattsson, K., Strassle, B., Trimmers, J. and Rhodes, K. (2000). Modulation of A-type Potassium Channels by a Family of Calcium Sensors. Nature, 403(6769):553-556.

Andrew, C., Bhattacharjee, S., Kokkoni, N., Hirst, J., Jones, G., and Doig, A. (2002). Stabilizing Interactions between Aromatic and Basic Side Chains in alpha Helical Peptides and Proteins. Tyrosine Effects on Helix Circular Dichroism. Journal of the American Chemical Society, 124(43):12706-12714.

Aravind, P., Chandra, K., Reddy, P., Jeromin, A., Chary, K., and Sharma, Y. (2008). Regulatory and Structural EF-hand Motifs of Neuronal Calcium Sensor-1: $\mathrm{Mg}^{2+}$ Modulates $\mathrm{Ca}^{2+}$ Binding, $\mathrm{Ca}^{2+}$ Induced Conformational Changes, and Equilibrium Unfolding Transitions. Journal of Molecular Biology, 376(4):1100-1115.

Arunachalam, J. and Gautham, N. (2008). Hydrophobic Clusters in Protein Structures. Proteins, 71(4): 2012-2025.

Augusteyn, R., Putilina, T., and Seifert, R. (1988). Quenching of Tryptophan Fluorescence in Bovine Lens Proteins by Acrylamide and Iodide. Current Eye Research, 7(3): 237-245.

Bai, X., Yan, C., Yang, G., Lu, P., Ma, D., Sun, L., Zhou, R., Scheres, S., and Shi, Y. (2015). An Atomic Structure of Human $\gamma$ Secretase. Nature, 525(7568):212-217.

Belogortseva, N., Rubio, M., Terrell, W. and Mikšovská, J. (2007). The Contribution of Heme Propionate Groups to the Conformational Dynamics Associated with CO Photo dissociation From Horse Heart Myoglobin. Journal of Inorganic Biochemistry, 101(7):977986.

Berova, N., Nakanishi, K., and Woody, R. (2000). Circular Dichroism-Principles and Application.

Berridge, M. (1997). Elementary and Global Aspects of Calcium Signaling. Journal of Experimental Biology, 200(2):315-319.

Berridge, M.J., LIPP, P. and Bootman, M.D., (2000). The versatility and universality of calcium signalling. Nature reviews.Molecular cell biology, 1(1): 11-21.

Berridge, M. (2001). The Versatility and Complexity of Calcium Signaling. Novartis Foundation Symposium, 239:52-64. 
Berridge, M., Bootman, M., and Roderick, H. (2003). Calcium Signaling: Dynamics, Homeostasis and Remodeling. Nature reviews. Molecular cell biology, 4(7):517-529.

Bhattacharya, S., Bunick, C., and Chazin, W. (2004). Target Selectivity in Ef-Hand Calcium Binding Proteins. Biochimica et Biophysica Acta (BBA) - Molecular Cell Research, 1742(1-3): 69-79.

Bismuto, E., Nucci, R., Rossi, M., and Irace, G. (1999). Structural and Dynamic Aspects of Beta-Glycosidase from Mesophilic and Thermophilic Bacteria by Multitryptophanyl Emission Decay Studies. Proteins, 35(2):163-172.

Bjelkmar, P., Larsson, P., Cuendet, M., Hess, B., and Lindal, E. (2010). Implementation of the CHARMM Force Field in GROMACS: Analysis of Protein Stability Effects from Correction Maps, Virtual Interaction Sites, and Water Models. Journal of Chemical Theory and Computation, 6(2):459-466.

Bodis, E., Raics, K., Nyitrai, M., Majer, Z., and Lukacs, A. (2013). Fluorescence Lifetime Distributions Report on Protein Destabilisation in Quenching Experiments. Journal of Photochemistry and Photobiology B: Biology, 129:108-114.

Boens, N., Qin, W., Basariä, N., Hofkens, J., Ameloot, M., Pouget, J., Lefevre, J., Valeur, B., Gratton, E., Vandeven, M., Silva, N., Engelborghs, Y., Willaert, K., Sillen, A., Rumbles, G., Phillips, D., Visser, A, Van Hoek, A., Lakowicz, J., Malak, H., Gryczynski, I., Szabo, A., Krajcarski, D., Tamai, N., and Miura, A. (2007). Fluorescence Lifetime Standards for Time and Frequency Domain Fluorescence Spectroscopy. Analytical Chemistry, 79(5):2137-2149.

Bourne, Y., Dannenberg, J., Pollmann, V., Marchot, P., and Pongs, O. (2001). Immunocytochemical Localization and Crystal Structure of Human Frequenin (Neuronal Calcium Sensor 1). Journal of Biological Chemistry, 276(15):11949-11955.

Braunewell, K. and Gundelfinger, E. (1999). Intracellular Neuronal Calcium Sensor Proteins: A Family of EF-Hand Calcium-Binding Proteins in Search of a Function. Cell and Tissue Research, 295(1):1-12.

Braunewell, K. and Klein-Szanto, A. (2009). Visinin-like proteins (VSNLs): Interaction Partners and Emerging Functions in Signal Transduction of a Subfamily of Neuronal $\mathrm{Ca}^{2+}$ Sensor Proteins. Cell and Tissue Research, 335(2):301-316.

Braunewell, K. (2005). The Darker Side of $\mathrm{Ca}^{2+}$ Signaling by Neuronal $\mathrm{Ca}^{2+}$ Sensor Proteins: From Alzheimer's disease to Cancer. Trends in Pharmacological Sciences, 26(7):345-351. 
Brochette, P., Petit, C. and Pileni, M. (1988). Cytochrome C in Sodium Bis(2-ethylhexyl) Sulfosuccinate Reverse Micelles: Structure and Reactivity. The Journal of Physical Chemistry, 92(12):3505-3511.

Bronner, F. and Petpulik, M. (1992). Extra-And Intracellular Calcium And Phosphate Regulation in: Basic Research to Clinical Medicine.

Brooks, B., Bruccoleri, R., Olafson, B., States, D., Swaminathan, S., and Karplus, M. (1983). CHARMM: A Program for Macromolecular Energy, Minimization, and Dynamics Calculations. Journal of Computational Chemistry, 4(2):187-217.

Burgoyne, R. (2007). Neuronal Calcium Sensor Proteins: Generating Diversity in Neuronal $\mathrm{Ca}^{2+}$ Signaling. Nature reviews.Neuroscience, 8(3):182-193.

Burgoyne, R. and Haynes, L. (2015). Sense and Specificity in Neuronal Calcium Signaling. Biochimica et Biophysica Acta, 1853(9):1921-1932.

Burgoyne, R. and Haynes, L. (2012). Understanding the Physiological Roles of the Neuronal Calcium Sensor Proteins. Molecular brain, DOI: 10.1186/1756-6606-5-2.

Burgoyne, R., O'callaghan, D., Hasdemir, B., Haynes, L., and Tepikin, A. (2004). Neuronal $\mathrm{Ca}^{2+}$ Sensor Proteins: Multitalented Regulators of Neuronal Function. Trends in Neurosciences, 27(4):203-209.

Burgoyne, R. and Weiss, J. (2001). The Neuronal Calcium Sensor Family of $\mathrm{Ca}^{2+}$ Binding Proteins. The Biochemical journal, 353(1):1-12.

Burley, S. and Petsko, G. (1985). Aromatic-Aromatic Interaction: A Mechanism of Protein Structure Stabilization. Science, 229(4708): 23-28.

Buxbaum, J. (2004). A Role for Calsenilin and Related Proteins in Multiple Aspects of Neuronal Function. Biochemical and Biophysical Research Communications, 322(4):1140-1144.

Buxbaum, J., Choi, E., Luo, Y., Lilliehool, C., Crowley, A., Merriam, D., and Wasco, W. (1998). Calsenilin: A Calcium-Binding Protein that Interacts with the Presenilins and Regulates the Levels of a Presenilin Fragment. Nature Medicine, 4(10):1177-1181.

Calloe, K., Cordeiro, J., Di Diego, J., Hansen, R., Grunnet, M., Olesen, S., and Antzelevitch, C. (2009). A Transient Outward Potassium Current Activator Recapitulates the Electrocardiographic Manifestations of Brugada Syndrome. Cardiovascular research, 81(4):686-694. 
Calvert, P., Klenchin, V. and Bownds, M. (1995). Rhodopsin Kinase Inhibition by Recoverin. Function of Recoverin Myristoylation. The Journal of Biological Chemistry, 270(41):24127-24129.

Carafoli, E., Santella, L., Branca, D., and Brini, M. (2001). Generation, Control, and Processing of Cellular Calcium Signals. Critical reviews in biochemistry and molecular biology, 36(2):107-260.

Carafoli, E. (2007). The Unusual History and Unique Properties of the Calcium Signal. New comprehensive biochemistry, 41: 3-22.

Carrion, A., Link, W., Ledo, F., Mellstrom, B., and Naranjo, J. (1999). DREAM is a Ca ${ }^{2+}$ Regulated Transcriptional Repressor. Nature, 398(6722):80-84.

Castro, G., Larson, B., Panilaitis, B.,and Kaplan, D.(2005). Emulsan Quantitation by Nile Red Quenching Fluorescence Assay. Applied Microbiology and Biotechnology, 67(6):767-770.

Cates, M., Berry, M., Ho, E., Li, Q., Potter, J., and Phillips JR, G. (1999). Metal-Ion Affinity and Specificity in EF-Hand Proteins: Coordination Geometry and Domain Plasticity in Parvalbumin. Structure, 7(10):1269-1278.

Chazin, W. (2011). Relating Form and Function of EF-Hand Calcium Binding Proteins. Accounts of Chemical Research, 44(3):171-179.

Chen, C., Inglese, J., Lefkowitz, R., and Hurley, J. (1995). $\mathrm{Ca}^{2+}$ Dependent Interaction of Recoverin with Rhodopsin Kinase. The Journal of Biological Chemistry, 270(30):1806018066.

Cheng, H., Pitcher, G., LaViolette, S., Whishaw, I., Tong, K., Kockeritz, L., Wada, T., Joza, N., Crackower, M., Goncalves, J., Sarosi, I., Woodgett, J., Oliveira-Dos-Santos, A., Ikura, M., Van, D., Salter, M., and Penninger, J. (2002). DREAM is a Critical Transcriptional Repressor for Pain Modulation. Cell, 108(1):31-43.

Chodera, J.and Mobley, D. (2013). Entropy-Enthalpy Compensation: Role and Ramifications in Biomolecular Ligand Recognition and Design. Annual Review of Biophysics, 42:121-142.

Clapham, E. D., (2007). Calcium Signaling. Cell, 131(6): 1047-1058.

Choi, E., Zaidi, N., Miller, J., Crowley, A., Merriam, D., Lilliehook, C., Buxbaum, J., and Wasco, W. (2001). Calsenilin is a Substrate for Caspase-3 that Preferentially Interacts with the Familial Alzheimer's Disease-Associated C-terminal Fragment of Presenilin 2. Journal of Biological Chemistry, 276(22):19197-19204. 
Chuang, S., Velkov, T., Horne, J., Porter, C., and Scanlon, M. (2008). Characterization of the Drug Binding Specificity of Rat Liver Fatty Acid Binding Protein. Journal of medicinal chemistry, 51(13):3755-3764.

Comeau, S., Gatchell, D., Vajda, S., and Camacho, C. (2004). ClusPro: a Fully Automated Algorithm for Protein-Protein Docking. Nucleic Acids Research, 32 (web server issue):W96-99.

Comeau, S., Gatchell, D., Vajda, S., and Camacho, C. (2004). ClusPro: An Automated Docking and Discrimination Method for the Prediction of Protein Complexes. Bioinformatics, 20(1):45-50.

Costigan, M. and Woolf, C. (2002). No DREAM, No Pain: Closing the Spinal Gate. Cell, 108(3):297-300.

Craig, T., Benson, L., Venyaminov, S.Y., Klimtchuk, E., Bajzer, Z., Prendergast, F., Naylor, S., and Kumar, R. (2002). The Metal Binding Properties of DREAM. Journal of Biological Chemistry, 277(13):10955-10966.

Culbertson, D. and Olson, J. (2010). Role of Heme in the Unfolding and Assembly of Myoglobin. Biochemistry, 49(29):6052-6063.

Burgoyne, R. (2004). The Neuronal Calcium-Sensor Proteins. Biochimica et Biophysica Acta (BBA) - Molecular Cell Research, 1742(1-3):59-68.

Da Silva, A., Kendrick-Jones, J., and Reinach, F. (1995). Determinants of Ion Specificity on EF-Hands Sites. Conversion of the $\mathrm{Ca}^{2+} / \mathrm{Mg}^{2+}$ Site of Smooth Muscle Myosin Regulatory Light Chain into a $\mathrm{Ca}^{2+}$ Specific Site. The Journal of biological chemistry, 270(12):6773-6778.

Dason, J., Romero-Pozuelo, J., Atwood, H., and Ferrus, A. (2012). Multiple Roles for Frequenin/Ncs-1 in Synaptic Function and Development. Molecular neurobiology, 45(2):388-402.

Datta, K. and Licata, V. (2003). Thermodynamics of the Binding of Thermus Aquaticus DNA polymerase to Primed-Template DNA. Nucleic acids research, 31(19):5590-5597.

Datta, K., Wowor, A., Richard, A., and Licata, V. (2006). Temperature Dependence and Thermodynamics of Klenow Polymerase Binding to Primed-Template DNA. Biophysical journal, 90(5):1739-1751.

Jameson, D. (2014). Introduction to Fluorescence.

De Vries, S., Van Dijk, M., and Bonvin, A. (2010). The HADDOCK Web Server for DataDriven Biomolecular Docking. Nature protocols, 5(5):883-897. 
Dewji, N. and Singer, S. (1997). The Seven-Transmembrane Spanning Topography of the Alzheimer Disease-Related Presenilin Proteins in the Plasma Membranes of cultured cells. Proceedings of the National Academy of Sciences of the United States of America, 94(25):14025-14030.

Diwu, Z., Lu, Y., Zhang, C., Klaubert, D., and Haugland, R. (1997). Fluorescent Molecular Probes II. The Synthesis, Spectral Properties and Use of Fluorescent Solvatochromic Dapoxyl Dyes. Photochemistry and photobiology, 66(4):424-431.

Dixit, S. and Beveridge, D. (2006). Structural Bioinformatics of DNA: A Web-Based Tool for the Analysis of Molecular Dynamics Results and Structure Prediction. Bioinformatics, 22(8):1007-1009.

Dizhoor, A., Ray, S., Kumar, S., Niemi, G., Spencer, M., Brolley, D., Walsh, K., Philipov, P., Hurley, J., and Stryer, L. (1991). Recoverin: A Calcium Sensitive Activator of Retinal Rod Guanylate Cyclase. Science, 251(4996):915-918.

Dizhoor, A., Lowe, D., Olshevskaya, E., Laura, R., and Hurley, J. (1994). The Human Photoreceptor Membrane Guanylyl Cyclase, RetGC, is Present in Outer Segments and is regulated by Calcium and a Soluble Activator. Neuron, 12(6):1345-1352.

Epps, D., Raub, T., and Kezdy, F. (1995). A General, Wide-Range Spectrofluorometric Method for Measuring the Site-Specific Affinities of Drugs toward Human Serum Albumin. Analytical Biochemistry, 227(2):342-350.

Falke, J., Drake, S., Hazard, A., and Peersen, O. (1994). Molecular Tuning of Ion Binding to Calcium Signaling Proteins. Quarterly reviews of biophysics, 27(03):219-290.

Fedrizzi, L., Lim, D., Carafoli, E., and Brini, M. (2008). Interplay of the Ca2+-Binding Protein DREAM with Presenilin in Neuronal $\mathrm{Ca}^{2+}$ Signaling. Journal of Biological Chemistry, 283(41):27494-27503.

Fenn, E., Wong, D., and Fayer, M. (2009). Water Dynamics at Neutral and Ionic Interfaces. Proceedings of the National Academy of Sciences, 106(36):15243-15248.

Fink, A. (1998). Protein Aggregation: Folding Aggregates, Inclusion Bodies and Amyloid. Folding and Design, 3(1):R9-R23.

Fischer, H., Polikarpov, I., and Craievich, A. (2004). Average Protein Density is a Molecular-Weight-Dependent Function. Protein Science: A Publication of the Protein Society, 13(10): 2825-2828.

Flaherty, K., Zozulya, S., Stryer, L., and Mckay, D. (1993). Three-Dimensional Structure of Recoverin, a Calcium Sensor in Vision. Cell, 75(4):709-716. 
Follenius-Wund, A., Piemont, E., Freyssinet, J., Gerard, D., and Pigault, C. (1997). Conformational Adaptation of Annexin V upon Binding to Liposomes: A Time-Resolved Fluorescence Study. Biochemical and biophysical research communications, 234(1):111116.

Freiburger, L., Auclair, K., and Mittermaier, A. (2009). Elucidating Protein Binding Mechanisms by Variable-c ITC. Chembiochem: a European Journal of Chemical Biology, 10(18): 2871-2873.

Furlong, W., Rubinski, M., and Indralingam, R. (2013). The Method of Continuous Variation: A Laboratory Investigation of the Formula of a Precipitate. Journal of Chemical Education, 90(7):937-940.

Gallagher, S. (2006). One-Dimensional SDS Gel Electrophoresis of Proteins. Current Protocols in Immunology. Chapter 8: Unit 8.4.

Garbett, N., Hammond, N., and Graves, D. (2004). Influence of the Amino Substituents in the Interaction of Ethidium Bromide with DNA. Biophysical journal, 87(6): 3974-3981.

Gibson, Q. (1969). Rapid mixing: Stopped flow. Methods in enzymology, 16:187-228.

Gifford, J., Walsh, M., and Vogel, H. (2007). Structures and Metal-Ion-Binding Properties of the $\mathrm{Ca}^{2+}$ Binding Helix-Loop-Helix EF-Hand Motifs. Biochemical Journal, 405(2):199221.

Girvan, M. and Newman, M. (2002). Community Structure in Social and Biological Networks. Proceedings of the National Academy of Sciences, 99(12): 7821-7826.

Gonzalez, W., Ramos, V., Diaz, M., Garabedian, A., Molano-Arevalo, J., Fernandez-Lima, F., and Miksovska, J. (2016). Characterization of the Photophysical, Thermodynamic, and Structural Properties of the Terbium (III)-DREAM Complex. Biochemistry, 55(12):18731886.

Gonzalez, W. and Miksovska, J. (2014). Application of ANS Fluorescent Probes to Identify Hydrophobic Sites on the Surface of DREAM. Biochimica et Biophysica Acta (BBA) Proteins and Proteomics, 1844(9):1472-1480.

Gonzalez, W., Pham, K., and Miksovska, J. (2014). Modulation of the voltage gated potassium channel (Kv4.3) and the auxiliary protein (KChIP3) interactions by the current activator NS5806. Journal of Biological Chemistry.

Gratton, E., Jameson, D., and Hall, R. (1984). Multifrequency Phase and Modulation Fluorometry. Annual Review of Biophysics and Bioengineering, 13:105-124. 
Greenfield, N. (2015). Circular dichroism (CD) Analyses of Protein-Protein Interactions. Methods in Molecular Biology, 1278:239-265.

Greenfield, N. (2006). Analysis of the Kinetics of Folding of Proteins and Peptides Using Circular Dichroism. Nature protocols, 1(6):2891-2899.

Greenfield, N. (2006). Using Circular Dichroism Collected as a Function of Temperature to Determine the Thermodynamics of Protein Unfolding and Binding Interactions. Nature protocols, 1(6):2527-2535.

Greenfield, N. (2006). Using Circular Dichroism Spectra to Estimate Protein Secondary Structure. Nature Protocols, 1(6):2876-2890.

Greenfield, N. (2004). Analysis of Circular Dichroism Data. Methods in Enzymology, 383:282-317.

Greenfield, N. (2004). Circular Dichroism Analysis for Protein-Protein Interactions. Methods in molecular biology, 261:55-78.

Handley, M., Lian, L., Haynes, L., and Burgoyne, R. (2010). Structural and Functional Deficits in a Neuronal Calcium Sensor-1 Mutant Identified in a Case of Autistic Spectrum Disorder. PLoS ONE. DOI: 10534.

Hargrove, M. (2005). Ligand Binding With Stopped-Flow Rapid Mixing. In: G. ULRICH NIENHAUS.Protein-Ligand Interactions: Methods and Applications: 323-341.

Hasinoff, B. (1974). Kinetic Activation Volumes of the Binding Of Oxygen and Carbon Monoxide to Hemoglobin and Myoglobin Studied on A High-Pressure Laser Flash Photolysis Apparatus. Biochemistry, 13(15):3111-3117.

Hawe, A., Rispens, T., Herron, J., and Jiskoot, W. (2011). Probing bis-ANS Binding Sites of Different Affinity on aggregated IgG by Steady-State Fluorescence, Time-Resolved Fluorescence and Isothermal Titration Calorimetry. Journal of pharmaceutical sciences, 100(4):1294-1305.

Haynes, L. and Burgoyne, R. (2008). Unexpected Tails of a $\mathrm{Ca}^{2+}$ Sensor. Nature chemical biology, 4(2):90-91.

Heidarsson, P., Bjerrum-Bohr, I., Jensen, G., Pongs, O., Finn, B., Poulsen, F., and Kragelund, B. (2012). The C-Terminal Tail of Human Neuronal Calcium Sensor 1 Regulates the Conformational Stability of the $\mathrm{Ca}^{2+}$ Activated State. Journal of Molecular Biology, 417(1-2):51-64. 
Heidarsson, P., Otazo, M., BelluccI, L., Mossa, A., Imparato, A., Paci, E., Corni, S., Di Felice, R., Kragelund, B. and Cecconi, C. (2013). Single-Molecule Folding Mechanism of an EF-Hand Neuronal Calcium Sensor. Structure, 21(10):1812-1821.

Henzl, M., Hapak, R., and Likos, J. (1998). Interconversion of the Ligand Arrays in the $\mathrm{CD}$ and $\mathrm{EF}$ Sites of Oncomodulin. Influence on $\mathrm{Ca}^{2+}$ Binding Affinity. Biochemistry, 37(25):9101-9111.

Holdgate, G. (2001). Making Cool Drugs Hot: Isothermal Titration Calorimetry as a Tool to Study Binding Energetics. BioTechniques, 31(1):164-170.

Holmqvist, M., Cao, J., Hernandez-Pineda, R., Jacobson, M., Carroll, K., Sung, M., Betty, M., Ge, P., Gilbride, K., Brown, M., Jurman, M., Lawson, D., Silos-Santiago, I., Xie, Y., Covarrubias, M., Rhodes, K., Distefano, P., and An, W. (2002). Elimination of Fast Inactivation in Kv4 A-type Potassium Channels by an Auxiliary Subunit Domain. Proceedings of the National Academy of Sciences, 99(2):1035-1040.

Hospital, A., Goã, J., Orozco, M., and Gelpã, J. (2015). Molecular Dynamics Simulations: Advances and Applications. Advances and Applications in Bioinformatics and Chemistry: AABC, 8:37-47.

Huang, H., Ishida, H., Yamniuk, A., and Vogel, H. (2011). Solution Structures of $\mathrm{Ca}^{2+} \mathrm{CIB} 1$ and $\mathrm{Mg}^{2+} \mathrm{CIB} 1$ and their Interactions with the Platelet Integrin AlphaIIb Cytoplasmic Domain. The Journal of Biological Chemistry, 286(19):17181-17192.

Humphrey, W., Dalke, A., and Schulten, K. (1996).VMD: Visual Molecular Dynamics. Journal of Molecular Graphics, 14(1): 33-38.

Hung, H. and Chang, G. (2001). Multiple Unfolding Intermediates of Human Placental Alkaline Phosphatase in Equilibrium Urea Denaturation. Biophysical Journal, 81(6): 34563471.

Hung, H., Chen, Y., Liu, G., Lee, H., and Chang, G. (2003). Equilibrium Protein FoldingUnfolding Process Involving Multiple Intermediates. Bulletin of mathematical biology, 65(4):553-570.

Ikura, M. (1996). Calcium Binding and Conformational Response in EF-Hand Proteins. Trends in Biochemical Sciences, 21(1):14-17.

Jang, C., Choi, J., Na, Y., Jang, B., Wasco, W., Buxbaum, J., Kim, Y., and Choi, E. (2011). Calsenilin Regulates Presenilin $1 \gamma$-secretase-mediated N-cadherin $\varepsilon$-cleavage and $\beta$ catenin Signaling. The FASEB Journal, 25(12):4174-4183. 
Jelesarov, I. and Bosshard, H. (1999). Isothermal Titration Calorimetry and Differential Scanning Calorimetry as Complementary Tools to Investigate the Energetics of Biomolecular Recognition. Journal of Molecular Recognition, 12(1):3-18.

Jerng, H., Pfaffinger, P., and Covarrubias, M. (2004). Molecular Physiology and Modulation of Somatodendritic A-Type Potassium Channels. Molecular and cellular neurosciences, 27(4):343-369.

Jeromin, A., Muralidhar, D., Parameswaran, M., Roder, J., Fairwell, T., Scarlata, S., Dowal, L., Mustafi, S., Chary, K., and Sharma, Y. (2004). N-terminal Myristoylation Regulates Calcium-induced Conformational Changes in Neuronal Calcium Sensor-1. Journal of Biological Chemistry, 279(26):27158-27167.

Jo, D., Chang, J., Hong, H., Mook-Jung, I., and Jung, Y. (2003). Contribution of Resenilin/gamma-secretase to Calsenilin-mediated Apoptosis. Biochemical and Biophysical Research Communications, 305(1):62-66.

Jo, D., Jang, J., Kim, B., Lundkvist, J., and Jung, Y. (2005). Overexpression of Calsenilin Enhances Gamma-Secretase Activity. Neuroscience Letters, 378(1):59-64.

Jo, D., Kim, M., Choi, Y., Kim, I., Song, Y., Woo, H., Chung, C., and Jung, Y. (2001). Pro-apoptotic Function of Calsenilin/DREAM/KChIP3. FASEB Journal: Official Publication of the Federation of American Societies for Experimental Biology, 15(3):589591.

Jo, D., Lee, J., Hong, Y., Song, S., Mook-Jung, I., Koh, J., and Jung, Y. (2004). Induction of pro-apoptotic Calsenilin/DREAM/KChIP3 in Alzheimer's disease and Cultured Neurons After Amyloid-beta Exposure. Journal of neurochemistry, 88(3):604-611.

Jo, D., Kim, M., Choi, Y., Kim, I., Song, Y., Woo, H., Chung, C., and Jung, Y. (2001). Pro-apoptotic Function of Calsenilin/DREAM/KChIP3. The FASEB Journal

Jo, S., Kim, T., Iyer, V., and Im, W. (2008). CHARMM-GUI: A Web-based Graphical User Interface for CHARMM. Journal of Computational Chemistry, 29(11):1859-1865.

Jobbágy, A., Jobbágy M. Examination of FITC Preparations. I. Measurements of the Dye Content of Fluorescein Isothiocyanate Preparations. (1973). Journal of immunological methods, 2.2: 159-68.

Lakowicz, J. (2010). Principles of Fluorescence Spectroscopy. USA: Springer.

Lakowicz, J. (2006). Principles of Fluorescence Spectroscopy, $3^{\text {rd }}$ edition.

Kelly, S., Jess, T., and Price, N. (2005). How to Study Proteins by Circular Dichroism. Biochimica et Biophysica Acta (BBA) -Proteins and Proteomics, 1751(2):119-139. 
Kotlarchyk, M., Huang, J., and Chen, S. (1985). Structure of AOT Reversed Micelles Determined By Small-Angle Neutron Scattering. The Journal of Physical Chemistry, 89(20): 4382 - 4386.

Kozakov, D., Brenke, R., Comeau, S., and Vajda, S. (2006). PIPER: an FFT-based Protein Docking Program with Pairwise Potentials. Proteins, 65(2): 392 - 406.

Kozakov, D., Beglov, D., Bohnuud, T., Mottarella, S., Xia, B., Hall, D., and Vajda, S. (2013). How good is Automated Protein Docking? Proteins: Structure, Function, and Bioinformatics, 81(12): 2159 - 2166.

Korson, L., Drost-Hansen, W., Millero, F.J. (1969). Viscosity of Water at various Temperatures, The Journal of physical chemistry, 73(1): 34 - 39.

Krieger, E., Koraimann, G., and Vriend, G. (2002). Increasing the Precision of Comparative Models with YASARA NOVA-A Self-Parameterizing Force Field. Proteins, 47(3):393 - 402.

Kuball, H. (1999). Circular Dichroism and Linear Dichroism. Zeitschrift für physikalische Chemie (Neue Folge), 212 (part_1): 118-119.

Ladbury, J. (2004). Application of Isothermal Titration Calorimetry in the Biological Sciences: Things Are Heating Up! BioTechniques, 37(6):885-887.

Ladbury, J. and Chowdhry, B. (1996). Sensing the Heat: The Application of Isothermal Titration Calorimetry to Thermodynamic Studies of Biomolecular Interactions. Chemistry \& biology, 3(10):791-801.

Lakowicz, J. and Netlibrary, I. (2002). Topics in fluorescence spectroscopy. New York: Kluwer Academic.

Larsen, R.W., Vetromile, C., Maza, W., Pham, K., and Miksovska, J. (2011). Exploring Biomolecular Thermodynamics in Aqueous and Non-Aqueous Environments using Time Resolved Photothermal Methods In: Juan M. Ruso and Angel Pineiro, ed, Proteins in Solution and at Interfaces. WILEY: 449-472.

Ledo, F., Carrion, A., Link, W., Mellstrom, B., and Naranjo, J. (2000). DREAMalphaCREM Interaction Via Leucine-Charged Domains Derepresses Downstream Regulatory Element-Dependent Transcription. Molecular and cellular biology, 20(24): 9120-9126.

Ledo, F., Kremer, L., Mellstrom, B., and Naranjo, J. (2002). Ca ${ }^{2+}$ Dependent Block of CREB-CBP Transcription by Repressor DREAM. The EMBO journal, 21(17): 4583-4592. 
Ledo, F., Link, W., Carrión, A., Echeverria, V., Mellström, B., and Naranjo, J. (2000). The DREAM-DRE Interaction: Key Nucleotides and Dominant Negative Mutants. Biochimica et Biophysica Acta (BBA) - Molecular Cell Research, 1498(2-3):162-168.

Lehmann, S., Chiesa, R., and Harris, D.A., 1997. Evidence for a Six-transmembrane Domain Structure of Presenilin 1. Journal of Biological Chemistry, 272(18):12047-12051.

Leissring, M., Yamasaki, T., Wasco, W., Buxbaum, J., Parker, I., and Laferla, F. (2000). Calsenilin Reverses Presenilin-Mediated Enhancement of Calcium Signaling. Proceedings of the National Academy of Sciences, 97(15):8590-8593.

Leon, R., Murray, J., Cragg, G., Farnell, B., West, N., Pace, T., Watson, P., Bohne, C., Boulanger, M., and Hof, F. (2009). Identification and Characterization of Binding Sites on S100a7, a Participant in Cancer and Inflammation Pathways. Biochemistry, 48(44):1059110600.

Li, X. and Greenwald, I. (1998). Additional Evidence for an Eight-TransmembraneDomain Topology for Caenorhabditis Elegans and Human Presenilins. Proceedings of the National Academy of Sciences of the United States of America, 95(12):7109-7114.

Li, Y., Bohm, C., Dodd, R., Chen, F., Qamar, S., Schmitt-Ulms, G., Fraser, P., and St George-Hyslop, P. (2014). Structural Biology of Presenilin 1 Complexes. Molecular Neurodegeneration, 9(1):1-10.

Lilliehook, C., Bozdagi, O., Yao, J., Gomez-Ramirez, M., Zaidi, N., Wasco, W., Gandy, S., Santucci, A., Haroutunian, V., Huntley, G., and Buxbaum, J. (2003). Altered Abeta Formation and Long-Term Potentiation in a Calsenilin Knock-Out. The Journal of Neuroscience: The Official Journal of the Society for Neuroscience, 23(27):9097-9106.

Lilliehook, C., Chan, S., Choi, E., Zaidi, N., Wasco, W., Mattson, M., and Buxbaum, J. (2002). Calsenilin Enhances Apoptosis by Altering Endoplasmic Reticulum Calcium Signaling. Molecular and Cellular Neuroscience, 19(4):552-559.

Lim, S., Dizhoor, A., and Ames, J. (2014). Structural Diversity of Neuronal Calcium Sensor Proteins and Insights for Activation of Retinal Guanylyl Cyclase by GCAP1. Frontiers in molecular neuroscience, $7: 19$.

Lim, S., Peshenko, I., Dizhoor, A., and Ames, J. (2009). Effects of $\mathrm{Ca}^{2+}, \mathrm{Mg}^{2+}$, and Myristoylation on Guanylyl Cyclase Activating Protein 1 Structure and Stability. Biochemistry, 48(5): 850 - 862.

Lindholm, D., Mercer, E., Yu, L., Chen, Y., Kukkonen, J., Korhonen, L., and Arumäe, U. (2002). Neuronal Apoptosis Inhibitory Protein: Structural Requirements for Hippocalcin Binding and Effects on Survival of NGF-dependent sympathetic neurons. Biochimica et Biophysica Acta (BBA) - Proteins and Proteomics, 1600(1): 138 - 147. 
Lu, X. and Olson, W.K. (2003). 3DNA: A Software Package for the Analysis, Rebuilding and Visualization of Three-Dimensional Nucleic Acid Structures. Nucleic acids research, 31(17): 5108 - 5121.

Lusin, J., Vanarotti, M., Dace, A., Li, C., Valiveti, A., and Ames, J. (2008). NMR Structure of DREAM: Implications for Ca2+-Dependent DNA Binding and Protein Dimerization. Biochemistry, 47(22): 6088 - 6088.

Lyskov, S. and Gray, J.J. (2008). The RosettaDock Server for Local Protein-Protein Docking. Nucleic acids research, 36:W233 - 238.

Ma, P., Rould, M., Weintraub, H., and Pabo, C. (1994). Crystal Structure of MyoD bHLH Domain-DNA Complex: Perspectives on DNA Recognition and Implications for Transcriptional Activation. Cell, 77(3): 451 - 459.

Mackerell, A. (2004). Empirical Force Fields for Biological Macromolecules: Overview and Issues. Journal of Computational Chemistry, 25(13): 1584 - 1604.

Makino, C., Dodd, R., Chen, J., Burns, M., Roca, A., Simon, M., and Baylor, D. (2004). Recoverin Regulates Light-Dependent Phosphodiesterase Activity In Retinal Rods. The Journal of General Physiology, 123(6): 729 -741.

Martinez, J., Murciano-Calles, J., Cobos, E., Iglesias-Bexiga, M., Luque, I., and Ruiz-Sanz, J. (2013). Isothermal Titration Calorimetry: Thermodynamic Analysis of the Binding Thermograms of Molecular Recognition Events by Using Equilibrium Models.

Mccue, H., Haynes, L., and Burgoyne, R. (2010). The Diversity of Calcium Sensor Proteins in the Regulation of Neuronal Function. Cold Spring Harbor Perspectives in Biology, 2(8)

McKinnon, I., Fall, L., Parody-Morreale, A., and Gill, S. (1984). A Twin Titration Microcalorimeter for the Study of Biochemical Reactions. Analytical Biochemistry, 139(1): 134 - 139.

Mcphalen, C., Strynadka, N., and James, M. (1991). Calcium-Binding Sites in Proteins: A Structural Perspective. Advances in Protein Chemistry, 42, 77-144.

Miksovska, J., Yom, J., Diamond, B., and Larsen, R. (2006). Spectroscopic and Photothermal Study of Myoglobin Conformational Changes in the Presence of Sodium Dodecyl Sulfate. Biomacromolecules, 7(2):476-482.

Moilanen, D., Levinger, N., Spry, D., and Fayer, M. (2007). Confinement or the Nature of the Interface? Dynamics of Nanoscopic Water. Journal of the American Chemical Society, 129(46):4311-14318. 
Moncrieffe, M., Juranic, N., Kemple, M., Potter, J., Macura, S., and Prendergast, F. (2000). Structure-Fluorescence Correlations in a Single Tryptophan Mutant of Carp Parvalbumin: Solution Structure, Backbone and Side-Chain Dynamics. Journal of Molecular Biology, 297(1):147-163.

Morohashi, Y., Hatano, N., Ohya, S., Takikawa, R., Watabiki, T., Takasugi, N., Imaizumi, Y., Tomita, T., and Iwatsubo, T. (2002). Molecular Cloning and Characterization of CALP/KChIP4, a Novel EF-hand Protein Interacting With Presenilin 2 and Voltage-Gated Potassium Channel Subunit Kv4. Journal of Biological Chemistry.

Mouawad, L., Maréchal, J., and Perahia, D. (2005). Internal Cavities and Ligand Passageways in Human Hemoglobin Characterized By Molecular Dynamics Simulations. Biochimica et Biophysica Acta (BBA) - General Subjects, 1724(3):385-393.

Murakami, H., Nishi, T., and Toyota, Y. (2011). Determination of Structural Parameters Of Protein-Containing Reverse Micellar Solution By Near-Infrared Absorption Spectroscopy. The Journal of Physical Chemistry.b, 115(19):5877-5885.

Muralidhar, D., Jobby, M., Krishnan, K., Annapurna, V., Chary, K., Jeromin, A., and Sharma, Y. (2005). Equilibrium Unfolding of Neuronal Calcium Sensor-1: N-Terminal Myristoylation Influences Unfolding And Reduces Protein Stiffening in the Presence of Calcium. Journal of Biological Chemistry, 280(16):15569-15578.

Nakai, T., Yamasaki, A., Sakaguchi, M., Kosaka, K., Mihara, K., Amaya, Y., and Miura, S. (1999). Membrane Topology of Alzheimer's disease-related Presenilin 1: Evidence for the Existence of A Molecular Species With a Seven Membrane-Spanning and One Membrane-Embedded Structure. Journal of Biological Chemistry, 274(33):23647-23658.

Naoe, K., Noda, K., Kawagoe, M., and Imai, M. (2004). Higher Order Structure of Proteins Solubilized in AOT Reverse Micelles. Colloids and surfaces.B, Biointerfaces, 38(3-4):179185.

Northrup, S. and Erickson, H. (1992). Kinetics of Protein-Protein Association Explained By Brownian Dynamics Computer Simulation. Proceedings of the National Academy of Sciences, 89(8):3338-3342.

O'Callaghan, D., Ivings, L., Weiss, J., Ashby, M., Tepikin, A., and Burgoyne, R. (2002). Differential Use of Myristoyl Groups on Neuronal Calcium Sensor Proteins as a Determinant of Spatio-temporal Aspects of $\mathrm{Ca}^{2+}$ Signal Transduction. Journal of Biological Chemistry, 277(16):14227-14237.

Ohki, S., Ikura, M., and Zhang, M. (1997). Identification of $\mathrm{Mg}^{2+}$ Binding Sites and the Role of $\mathrm{Mg}^{2+}$ on Target Recognition by Calmodulin. Biochemistry, 36(14):4309-4316. 
Olson, E.J. and Bãhlmann, P. (2011). Getting More out of a Job Plot: Determination of Reactant to Product Stoichiometry in Cases of Displacement Reactions and n:n Complex Formation. The Journal of organic chemistry, 76(20):8406-8412.

Osawa, M., Tong, K., Lilliehook, C., Wasco, W., Buxbaum, J., Cheng, H., Penninger, J., Ikura, M. and Ames, J. (2001). Calcium-regulated DNA Binding and Oligomerization of the Neuronal Calcium-Sensing Protein, Calsenilin/DREAM/KChIP3. The Journal of Biological Chemistry, 276(44):41005-41013.

Osawa, M., Dace, A., Tong, K., Valiveti, A., Ikura, M., and Ames, J. (2005). $\mathrm{Mg}^{2+}$ and $\mathrm{Ca}^{2+}$ Differentially Regulate DNA Binding and Dimerization of DREAM. Journal of Biological Chemistry, 280(18):18008-18014.

Palaniappan, V. and Bocian, D. (1994). Acid-Induced Transformations of Myoglobin. Characterization of a New Equilibrium Heme-Pocket Intermediate. Biochemistry, 33(47):14264-14274.

Periasamy, A. and Clegg, R. (2010). FLIM Microscopy in Biology and Medicine. Boca Raton, FL, USA: Taylor and Francis Group, LLC.

Peshenko, I. and Dizhoor, A. (2006). $\mathrm{Ca}^{2+}$ and $\mathrm{Mg}^{2+}$ Binding Properties of GCAP-1: Evidence that $\mathrm{Mg}^{2+}$ Bound Form is the Physiological Activator of Photoreceptor Guanylyl Cyclase. Journal of Biological Chemistry, 281(33):23830-23841.

Peshenko, I. and Dizhoor, A. (2004). Guanylyl Cyclase-activating Proteins (GCAPs) are $\mathrm{Ca}^{2+} / \mathrm{Mg}^{2+}$ Sensors: Implications for Photoreceptor Guanylyl Cyclase (RetGC) Regulation in Mammalian Photoreceptors. Journal of Biological Chemistry, 279(17):16903-16906.

Pham, K., Dhulipala, G., Gonzalez, W., Gerstman, B., Regmi, C., Chapagain, P., and Miksovska, J. (2015). $\mathrm{Ca}^{2+}$ and $\mathrm{Mg}^{2+}$ Modulate Conformational Dynamics And Stability Of Downstream Regulatory Element Antagonist Modulator. Protein science: A publication of the Protein Society, 24(5):741-751.

Pham, K. and Miksovska, J. (2016). Molecular insight of DREAM and presenilin $1 \mathrm{C}$ terminal fragment interactions. FEBS letters, 590(8):1114-1122.

Phillips, J., Braun, R., Wang, W., Gumbart, J., Tajkhorshid, E., Villa, E., Chipot, C., Skeel, R., Kale, L., and Schulten, K. (2005a). Scalable Molecular Dynamics with NAMD. Journal of computational chemistry, 26(16):1781-1802.

Phillips, J., Braun, R., Wang, W., Gumbart, J., Tajkhorshid, E., Villa, E., Chipot, C., Skeel, R., Kalé, L., and Schulten, K. (2005b). Scalable Molecular Dynamics with NAMD. Journal of Computational Chemistry, 26(16):1781-1802. 
Pierce, B., Wiehe, K., Hwang, H., Kim, B., Vreven, T., and Weng, Z. (2014). ZDOCK server: Interactive Docking Prediction of Protein-Protein Complexes and Symmetric Multimers. Bioinformatics.

Pioletti, M., Findeisen, F., Hura, G., and Minor, D. (2006). Three-Dimensional Structure of the KChIP1-Kv4.3 T1 Complex Reveals a Cross-Shaped Octamer. Nature structural \& molecular biology, 13(11):987-995.

Podlisny, M., Citron, M., Amarante, P., Sherrington, R., Xia, W., Zhang, J., Diehl, T., Levesque, G., Fraser, P., Haass, C., Koo, E., Seubert, P., St George-Hyslop, P., Teplow, D., and Selkoe, D. (1997). Presenilin Proteins Undergo Heterogeneous Endoproteolysis between Thr291 and Ala299 and Occur As Stable N- And C-Terminal Fragments in Normal and Alzheimer Brain Tissue. Neurobiol Dis, 3.

Pongs, O., Lindemeier, J., Zhu, X., Theil, T., Engelkamp, D., Krah-jentgens, I., Lambrecht, H., Koch, K.W., Schwemer, J., Rivosecchi, R., Mallart, A., Galceran, J., Canal, I., Barbas, J., and Ferrús, A. (1993). Frequenin-A Novel Calcium-Binding Protein That Modulates Synaptic Efficacy in The Drosophila Nervous System. Neuron, 11(1):15-28.

Privalov, P. and Ebrary, I. (2012). Microcalorimetry of macromolecules the physical basis of biological structures. Hoboken, N.J: Wiley.

Puchalski, M., Morra, M., and Von, W. (1991). Assessment of Inner Filter Effect Corrections in Fluorimetry. Fresenius' Journal of Analytical Chemistry, 340(6):341-4.

Ramachandran, P., Craig, T., Atanasova, E., Cui, G., Owen, B., Bergen, H., MER, G., and Kumar, R. (2012). The Potassium Channel Interacting Protein 3 (DREAM/KChIP3) Heterodimerizes with and Regulates Calmodulin Function. The Journal of biological chemistry, 287(47):39439-39448.

Romani, A. and Scarpa, A. (1992). Regulation of Cell Magnesium. Archives of Biochemistry and Biophysics, 298(1):1-12.

Ross, J. and Jameson, D. (2008). Time-Resolved Methods in Biophysics.8. Frequency Domain Fluorometry: Applications to Intrinsic Protein Fluorescence. Photochemical \& photobiological sciences: Official journal of the European Photochemistry Association and the European Society for Photobiology, 7(11):1301-1312.

Rost, B. and Sander, C. (1994). Conservation and Prediction of Solvent Accessibility in Protein Families. Proteins, 20(3):216-226.

Santoro, M. and Bolen, D. (1988). Unfolding free Energy Changes Determined by the Linear Extrapolation Method. 1. Unfolding of Phenylmethanesulfonyl Alpha.Chymotrypsin Using Different Denaturants. Biochemistry, 27(21):8063-8068. 
Sanz C., Mellstrom B., Link W.A., Naranjo J.R., Fernandez-Luna J.L., Interleukin 3dependent activation of DREAM is involved in transcriptional silencing of the apoptotic Hrk gene in hematopoietic progenitor cells. The EMBO journal 20.9 (2001): 2286-2292

Scannevin, R., Wang, K., Jow, F., Megules, J., Kopsco, D., Edris, W., Carroll, K., Lü, Q., Xu, W., Xu, Z., Katz, A., Olland, S., Lin, L., Taylor, M., Stahl, M., Malakian, K., Somers, W., Mosyak, L., Bowlby, M., Chanda, P., and Rhodes, K. (2004). Two N-Terminal Domains of $\mathrm{Kv} 4 \mathrm{~K}^{+}$Channels Regulate Binding to and Modulation by KChIP1. Neuron, 41(4):587-598.

Schrodinger, L. (2015). The PyMOL Molecular Graphics System, Version 1.7.4.

Schwenk, J., Zolles, G., Kandias, N., Neubauer, I., Kalbacher, H., Covarrubias, M., Fakler, B., and Bentrop, D. (2008). NMR Analysis of KChIP4a Reveals Structural Basis for Control of Surface Expression of Kv4 Channel Complexes. Journal of Biological Chemistry, 283(27):18937-18946.

Sethi, A., Eargle, J., Black, A., and Luthey-Schulten, Z. (2009). Dynamical Networks in tRNA: Protein Complexes. Proceedings of the National Academy of Sciences of the United States of America, 106(16): 6620-6625.

Skelton, N., Kordel, J., Akke, M., Forsen, S., and Chazin, W. (1994). Signal Transduction versus Buffering Activity in $\mathrm{Ca}^{2+}$ Binding Proteins. Nature structural biology, 1(4):239245.

Small, J., Libertini, L., and Small, E. (1992). Analysis of Photoacoustic Waveforms Using the Nonlinear Least Squares Method. Biophysical chemistry, 42(1):29-48.

Smith, P., Krohn, R., Hermanson, G., Mallia, A., Gartner, F., Provenzano, M., Fujimoto, E., Goeke, N., Olson, B., and Klenk, D. (1985). Measurement of Protein Using Bicinchoninic Acid. Analytical Biochemistry, 150(1):76-85.

Sobhanifar, S., Schneider, B., Löhr, F., Gottstein, D., Ikeya, T., Mlynarczyk, K., Pulawski, W., Ghoshdastider, U., Kolinski, M., Filipek, S., Güntert, P., Bernhard, F., and Dötsch, V. (2010). Structural Investigation of the C-Terminal Catalytic Fragment of Presenilin 1. Proceedings of the National Academy of Sciences, 107(21):9644-9649.

Song, Z., Zheng, X., and Yang, B. (2013). Conformational Stability of CopC and Roles of Residues Tyr79 and Trp83. Protein science: a publication of the Protein Society, 22(11):1519-1530.

Spasic, D., Tolia, A., Dillen, K., Baert, V., De Strooper, B., Vrijens, S., and Annaert, W. (2006). Presenilin-1 Maintains a Nine-Transmembrane Topology Throughout the Secretory Pathway. Journal of Biological Chemistry, 281(36): 26569-26577. 
Spilker, C., Gundelfinger, E., and Braunewell, K. (2002). Evidence for Different Functional Properties of the Neuronal Calcium Sensor Proteins VILIP-1 and VILIP-3: from Subcellular Localization to Cellular Function. Biochimica et Biophysica Acta (BBA) Proteins and Proteomics, 1600(1-2):118-127.

Stegemeyer, H. (1969). Photoluminescence of Solutions. Angewandte Chemie, 81(23):1007-1008.

Stern V. and Volmer M. (1919). On the quenching-time of fluorescence. Physik Zeitschr, 20:183-188.

Strynadka, N. and James, M. (1989). Crystal Structures of the Helix-Loop-Helix CalciumBinding Proteins. Annual Review of Biochemistry, 58(1):951-999.

Szabo, A. and Rayner, D. (1980). Fluorescence Decay of Tryptophan Conformers in Aqueous Solution. Journal of the American Chemical Society, 102(2):554-563.

Tanaka, T., Ames, J., Harvey, T., Stryer, L., and Ikura, M. (1995). Sequestration of the Membrane-Targeting Myristoyl Group of Recoverin in the Calcium-Free State. Nature, 376(6539):444-447.

Togashi, D., Ryder, A., and O'Shaughnessy, D. (2010). Monitoring Local Unfolding of Bovine Serum Albumin During Denaturation Using Steady-State and Time-Resolved Fluorescence Spectroscopy. Journal of Fluorescence, 20(2):441-452.

Upadhyay, A., Bhatt, T., Tripathi, H.B. \& Pant, D.D. (1995). Photophysics of 8anilinonaphthalene-1-sulphonate. Journal of Photochemistry and Photobiology A: Chemistry, 89 (3): 201-207

Valentine, K., Peterson, R., Saad, J., Summers, M., Xu, X., Ames, J., and Wand, A. (2010). Reverse Micelle Encapsulation of Membrane-Anchored Proteins for Solution NMR Studies. Structure, 18(1):9-16.

Valeur, B. (2002). Molecular Fluorescence Principles and Applications. Weinheim, Germany: Wiley-VCH Verlad GmbH.

Van Dijk, M. and Bonvin, A. (2009). 3D-DART: a DNA Structure Modelling Server. Nucleic acids research, 37(suppl 2):W235-W239.

Van Dijk, M., Van Dijk, A., Hsu, V., Boelens, R., and Bonvin, A. (2006). InformationDriven Protein-DNA Docking Using HADDOCK: it is a Matter of Flexibility. Nucleic acids research, 34(11):3317-3325. 
Van Horn, W., Ogilvie, M., and Flynn, P. (2009). Reverse Micelle Encapsulation as a Model for Intracellular Crowding. Journal of the American Chemical Society, 131(23):8030-8039.

Van Horn, W., Simorellis, A., and Flynn, P. (2005). Low-Temperature Studies of Encapsulated Proteins. Journal of the American Chemical Society, 127(39):13553-13560.

Velazquez-Campoy, A., Leavitt, S., and Freire, E. (2004). Characterization of ProteinProtein Interactions by Isothermal Titration Calorimetry. Methods in molecular biology (Clifton, N.J.), 261:35-54.

Verbaro, D., Hagarman, A., Kohli, A., and Schweitzer-Stenner, R. (2009). Microperoxidase 11: A Model System for Porphyrin Networks and Heme Protein Interactions. JBIC Journal of Biological Inorganic Chemistry, 14(8):1289-1300.

Vijay-Kumar, S. and Kumar, V. (1999). Crystal Structure of Recombinant Bovine Neurocalcin. Nature Structural Biology, 6(1):80-88.

Vogel, H., Brokx, R., and Ouyang, H. (2002). Calcium-Binding Proteins. In: Hans J. Vogel, ed, Calcium-Binding Protein Protocols. Humana Press: 3-20.

Vogel, H. (1994). The Merck Frosst Award Lecture 1994. Calmodulin: A Versatile Calcium Mediator Protein. Biochemistry and cell biology Biochimie et biologie cellulaire, 72(9-10):357-376.

Wang, H., Yan, Y., Liu, Q., Huang, Y., Shen, Y., Chen, L., Chen, Y., Yang, Q., Hao, Q., Wang, K., and Chai, J.(2007). Structural Basis for Modulation of kv4 K ${ }^{+}$Channels by Auxiliary kchip Subunits. Nature neuroscience, 10(1): 32-39.

Wang, J. and Van Wart, H. (1989). Resonance Raman Characterization of The Heme c Group in N-acetyl microperoxidase-8: a Thermal Intermediate Spin-High Spin State Mixture. The Journal of physical chemistry, 93(23):7925-7931.

Wassenaar, T., Dijk, M., Loureiro-Ferreira, N., Schot, G., Vries, S., Schmitz, C., Zwan, J., Boelens, R., Giachetti, A., Ferella, L., Rosato, A., Bertini, I., Herrmann, T., Jonker, H., Bagaria, A., Jaravine, V., Gãntert, P., Schwalbe, H., Vranken, W., Doreleijers, J., Vriend, G., Vuister, G., Franke, D., Kikhney, A., Svergun, D., Fogh, R., Ionides, J., Laue, E., Spronk, C., Jurkåa, S., Verlato, M., Badoer, S., Dal Pra, S., Mazzucato, M., Frizziero, E., and Bonvin, A. (2012). WeNMR: Structural Biology on the Grid. Journal of Grid Computing, 10(4):743-767.

Weber, G. (1953). Rotational Brownian motion and Polarization of the Fluorescence of Solutions. Advances in Protein Chemistry, 8:415-459. 
Weber, G. (1977). Theory of Differential Phase Fluorometry: Detection of Anisotropic Molecular Rotations. The Journal of chemical physics, 66(9):4081-4091.

Wiechelman, K., Braun, R., and Fitzpatrick, J. (1988). Investigation of the Bicinchoninic Acid Protein Assay: Identification of the Groups Responsible For Color Formation. Analytical Biochemistry, 175(1):231-237.

Williams, A., Winfield, S., and Miller, J. (1983). Relative Fluorescence Quantum Yields Using a Computer-Controlled Luminescence Spectrometer. Analyst, 108(1290):10671071.

Wingard, J., Chan, J., Bosanac, I., Haeseleer, F., Palczewski, K., Ikura, M., and Ames, J. (2005). Structural Analysis of $\mathrm{Mg} 2+$ and $\mathrm{Ca} 2+$ Binding to CaBP1, a Neuron-specific Regulator of Calcium Channels. Journal of Biological Chemistry, 280(45):37461-37470.

Wurth, C., Grabolle, M., Pauli, J., Spieles, M., and Resch-Genger, U. (2013). Relative and Absolute Determination of Fluorescence Quantum Yields of Transparent Samples. Nat.Protocols, 8(8):1535-1550.

Yamniuk, A., Nguyen, L., Hoang, T., and Vogel, H. (2004). Metal Ion Binding Properties and Conformational States of Calcium- and Integrin-Binding Protein. Biochemistry, 43(9):2558-2568.

Yu, L., Sun, C., Mendoza, R., Wang, J., Matayoshi, E., Hebert, E., Pereda-Lopez, A., Hajduk, P., and Olejniczak, E. (2007). Solution Structure and Calcium-Binding Properties of EF-Hands 3 and 4 of Calsenilin. Protein Science, 16(11):2502-2509.

Zhou, J., Liyanage, U., Medina, M., Ho, C., Simmons, A., Lovett, M., and Kosik, K. (1997). Presenilin 1 Interaction in the Brain with a Novel Member of the Armadillo Family. Neuroreport, 8(8):2085-2090.

Zhou, W., Qian, Y., Kunjilwar, K., Pfaffinger, P., and Choe, S. (2004). Structural Insights into The Functional Interaction of KChIP1 with Shal-type $K^{+}$channels. Neuron, 41(4):573586.

Zinsli, P. (1979). Inhomogeneous interior of aerosol ot microemulsions probed by fluorescence and polarization decay. The Journal of physical chemistry, 83(25):32233231. 
VITA

KHOA NGOC PHAM

EDUCATION

2010

BSc. in Chemistry

Florida International University

Miami, Florida USA

$2011-2016$

Doctoral Candidate in Chemistry

Florida International University

Miami, Florida USA

AWARDS

2016

Dissertation Year Fellowship Award

2014

Summer Research Award, NIH Biomedical

Research Initiative

2013

Graduate Poster Presentation $2^{\text {nd }}$ prize, Graduate Symposium, Miami, FL

2013

ACS South Florida Travel Award, American Chemical Society

2010

Undergraduate Outstanding Academic Achievement in Chemistry Award

2009

Undergraduate Poster Presentation $1^{\text {st }}$ prize, Florida Annual Meeting and Exposition

\section{PUBLICATIONS}

MOLECULAR INSIGHT OF DREAM AND PRESENILIN 1 C-TERMINAL FRAGMENT INTERACTIONS. Khoa Pham and Jaroslava Miksovska. FEBS Letters, (2016), 590(8):1114-1122.

$\mathrm{CA}^{2+} \mathrm{AND}^{\mathrm{MG}^{2+}}$ MODULATE CONFORMATIONAL DYNAMICS AND STABILITY OF DOWNSTREAM REGULATORY ELEMENT ANTAGONIST MODULATOR. Khoa Pham, Gangadhar Dhulipala, Walter G. Gonzalez, Bernard S. Gerstman. Chola Regmi, Prem P. Chapagain, and Jaroslava Miksovska. Protein Science, (2015), 24: 741-751. 
CHARACTERIZATION OF MOLECULAR MECHANISM OF NEUROGLOBIN BINDING TO CYTOCHROME C: A SURFACE PLASMON RESONANCE AND ISOTHERMAL TITRATION CALORIMETRY STUDY. Purushottam Babu Tiwari, Luisana Astudillo, Khoa Pham, Xuewen Wang, Jin He, Sophie Bernad, Valérie Derrien, Pierre Sebban, Jaroslava Miksovska, and Yesim Darici. Inorganic Chemistry Communication, (2015), 62: 37 - 41.

MODULATION OF THE VOLTAGE-GATED POTASSIUM CHANNEL (Kv4.3) AND THE AUXILIARY PROTEIN (KCHIP3) INTERACTIONS BY THE CURRENT ACTIVATOR NS5806. Walter G. Gonzalez, Khoa Pham, Jaroslava Miksovska. Journal of Biological Chemistry, (2013), 289: 32201 - 32213.

EXPLORING BIOMOLECULAR THERMODYNAMICS IN AQUEOUS AND NONAQUEOUS ENVIRONMENTS USING TIME RESOLVED PHOTOTHERMAL METHODS. PROTEINS IN SOLUTIONS AND INTERFACES: METHODS AND APPLICATIONS IN BIOTECHNOLOGY AND MATERIAL SCIENCES. Randy W. Larsen, Carissa M. Vetromille, William A. Maza, Khoa Pham, and Jaroslava Miksovska. John Wiley and Sons Inc., (2011), Editors: J.M. Ruso and A. Pineiro, Chapter 23.

$\mathrm{CA}^{2+}$ AND $\mathrm{MG}^{2+}$ BINDING TO THE C-TERMINAL DOMAIN MODULATES DREAM PROTEIN STRUCTURE DYNAMICS AND STABILITY. Khoa Pham, Walter G. Gonzalez, and Jaroslava Miksovska. (Expected 2016).

THE FUNCTIONAL ROLES OF EF-HANDS IN DREAM IN MODULATING THE CONFORMATIONAL DYNAMICS AND INTERACTION WITH DNA. Khoa Pham, Jose Alfonso, Maria Santiago, and Jaroslava Miksovska. (Expected 2016).

CHARACTERIZATION OF THE ROLE OF INDIVIDUAL EF-HANDS IN DREAM IN MODULATING CONFORMATIONAL DYNAMICS, OLIGOMERIC STATES, AND INTERACTION WITH DNA. Khoa Pham, Jose Alfonso, and Jaroslava Miksovska, (2016), Biophysical Society $60^{\text {th }}$ Annual Meeting, Hollywood, CA.

IDENTIFY THE BINDING INTERFACE BETWEEN CALSENILIN AND PRESENILIN 1 C-TERMINAL FRAGMENT. Khoa Pham and Jaroslava Miksovska, (2015), Biophysical Society $59^{\text {th }}$ Annual Meeting, Baltimore, MD.

SPECTROSCOPIC STUDY CA ${ }^{2+}$ INDUCED CHANGES IN THE STRUCTURE, DYNAMICS AND STABILITY OF DREAM PROTEIN AND ITS MECHANISM OF DNA INTERACTION. Khoa Pham and Jaroslava Miksovska, (2013), Biophysical Society $57^{\text {th }}$ Annual Meeting, Philadelphia, PA. 